\title{
Palladium-Catalyzed Direct Dicarbonylation of Amines with Ethylene to Imides
}

Chang-Sheng Kuai ${ }^{[a]}$, Le-Cheng Wang ${ }^{[a]}$, Jian-Xing Xu ${ }^{[a]}$ and Xiao-Feng Wu ${ }^{[a] b b^{*}}$

[a] Da lian National Laboratory for Clean Energy, Da lian Institute of Chemical Physics, Chinese Academy of Sciences, 116023 Dalian, Liaoning, China

[b] Leibniz-Institut für Katalyse e. V., Albert-Einstein-Straße 29a, 18059Rostock, Germany

\section{Table of Contents}

1. General experimental details.................................................................................................................. S2

2. Typical procedure for Pd-catalyzed dicarbonylation of amines with ethylene ........................S3

3. Typical procedure for Pd-catalyzed carbonylation of alcohols with ethylene.......................... S3

4. Spectroscopic Data of Products.................................................................................................................... S5

5. References......................................................................................................................................................520

6. NMR Spectra of the Products ..............................................................................................522 


\section{General experimental details}

Unless otherwise noted, all reactions were carried out under a carbon monoxide or nitrogen atmosphere. The amines and reagents were ordered from Adamas-beta ${ }^{\circledR}$, Energy Chemical Sigma-Aldrich, Bidepharm and used without purification. All solvents were dried by standard techniques and distilled prior to use. Column chromatography was performed on silica gel (200-300 meshes). All NMR spectra were recorded at ambient temperature using Bruker Avance III $400 \mathrm{MHz}$ NMR $\left({ }^{1} \mathrm{H}, 400 \mathrm{MHz} ;{ }^{13} \mathrm{C}\left\{{ }^{1} \mathrm{H}\right\}, 101 \mathrm{MHz},{ }^{19} \mathrm{~F} 376 \mathrm{MHz}\right)$, Bruker AVANCE III HD 700MHz NMR spectrometers $\left({ }^{1} \mathrm{H}, 700 \mathrm{MHz} ;{ }^{13} \mathrm{C}\left\{{ }^{1} \mathrm{H}\right\}, 176 \mathrm{MHz}\right) .{ }^{1} \mathrm{H}$ NMR chemical shifts are reported relative to TMS and were referenced via residual proton resonances of the corresponding deuterated solvent $\left(\mathrm{CDCl}_{3}: 7.26 \mathrm{ppm}\right)$ wherea s ${ }^{13} \mathrm{C}\left\{{ }^{1} \mathrm{H}\right\}$ NMR spectra are reported relative to TMS via the carbon signals of the deuterated solvent $\left(\mathrm{CDCl}_{3}: 77.0 \mathrm{ppm}\right)$. Data for ${ }^{1} \mathrm{H}$ are reported as follows: chemical shift $(\delta \mathrm{ppm})$, multiplicity $(\mathrm{s}=$ singlet, $\mathrm{d}=$ doublet, $\mathrm{t}=$ triplet, $\mathrm{q}$ $=$ quartet, quint $=$ quintet, $\mathrm{m}=$ multiplet, $\mathrm{br}=$ broad $)$, coupling constant $(\mathrm{Hz})$, and integration. All ${ }^{13} \mathrm{C}$ NMR spectra were broad-band ${ }^{1} \mathrm{H}$ decoupled. All reactions were monitored by GC-FID or NMR analysis. HRMS data was obtained with Micromass HPLC-Q-TOF mass spectrometer (ESI) or Agilent 6540 Accurate-MS spectrometer (Q-TOF).

Because of the high toxicity of carbon monoxide, all the reactions should be performed in an autoclave. The laboratory should be well-equipped with a $\mathrm{CO}$ detector and alarm system. 


\section{Typical procedure for Pd-catalyzed dicarbonylation of amines with ethylene}

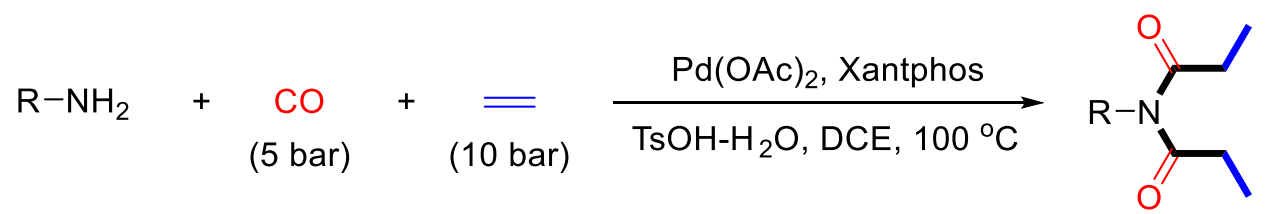

A $4 \mathrm{~mL}$ screw-cap vial was charged with $\mathrm{Pd}\left(\mathrm{PPh}_{3}\right)_{4}(11.6 \mathrm{mg}, 2.5 \mathrm{~mol} \%)$, xantphos (5.8 mg, $2.5 \mathrm{~mol} \%), p$-TSA (3.8 mg, $5 \mathrm{~mol} \%$ ) and an oven-dried stir bar. The vial was closed with a Teflon septum and cap and connected to the atmosphere via a needle. After DCE (1.5 mL), amine $(0.4 \mathrm{mmol})$ were added with a syringe under argon atmosphere, the vial was moved to an alloy plate and put into a Parr 4560 series autoclave $(300 \mathrm{~mL})$ under an argon atmosphere. At room temperature, the a utoclave was flushed with $\mathrm{CO}$ three times and charged with 5 bar of $\mathrm{CO}$ and 10 bar of ethylene. The autoclave was placed on a heating plate equipped with a magnetic stirrer and an a luminum block. The reaction mixture was heated to $100{ }^{\circ} \mathrm{C}$ (aluminum block) for $16 \mathrm{~h}$. After the reaction was complete, the autoclave was cooled down with ice water to room temperature and the pressure was released carefully. After cooling to room temperature, the reaction mixture was directly purified by column chromatography on silica gel using petroleum and ethyl acetate to afford the corresponding imide product.

$2 \mathrm{mmol}$ scale: A $20 \mathrm{~mL}$ screw-cap vial was charged with $\mathrm{Pd}\left(\mathrm{PPh}_{3}\right)_{4}(57.8 \mathrm{mg}, 2.5 \mathrm{~mol} \%)$, xantphos (28.9 mg, $2.5 \mathrm{~mol} \%)$, PTSA (19 mg, $5 \mathrm{~mol} \%$ ) and an oven-dried stir bar. The vial was closed with a Teflon septum and cap and connected to the atmosphere via a needle. After DCE $(7.5 \mathrm{~mL})$, Aniline $(186.3 \mathrm{mg}, 2.0 \mathrm{mmol})$ were added with a syringe under argon a tmosphere, the vial was moved to an alloy plate and put into a Parr 4560 series autoclave $(300 \mathrm{~mL})$ under an argon atmosphere. At room temperature, the autoclave was flushed with $\mathrm{CO}$ three times and charged with 5 bar of $\mathrm{CO}$ and 10 bar of ethylene. The autoclave was placed on a heating plate equipped with a magnetic stirrer and an aluminum block. The reaction mixture was heated to 100 ${ }^{\circ} \mathrm{C}$ (aluminum block) for $16 \mathrm{~h}$. After the reaction was complete, the autoclave wa s cooled down with ice water to room temperature and the pressure was released carefully. After cooling to room temperature, the reaction mixture was directly purified by column chromatography on silica gel using petroleum and ethylacetate $(\mathrm{PE}: \mathrm{EA}=10: 1)$ to afford imide product $0.368 \mathrm{~g}, 90 \%$.

\section{Typical procedure for Pd-catalyzed carbonylation of alcohols with ethylene}

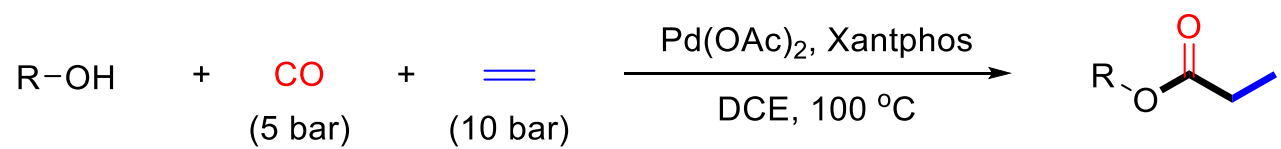

A $4 \mathrm{~mL}$ screw-cap vial was charged with $\mathrm{Pd}\left(\mathrm{PPh}_{3}\right)_{4}(11.6 \mathrm{mg}, 2.5 \mathrm{~mol} \%)$, xantphos (5.8 mg, $2.5 \mathrm{~mol} \%$ ) and an oven-dried stir bar. The vial was closed with a Teflon septum and cap and connected to the atmosphere via a needle. After DCE $(1.5 \mathrm{~mL})$, alcohol $(0.4 \mathrm{mmol})$ were added with a syringe under argon atmosphere, the vial was moved to an alloy plate and put into a Parr 4560 series autoclave $(300 \mathrm{~mL})$ under an argon atmosphere. At room temperature, the autoclave was flushed with $\mathrm{CO}$ three times and charged with 5 bar of $\mathrm{CO}$ and 10 bar of ethylene. The autoclave was placed on a heating plate equipped with a ma gnetic stirrer and a n aluminum block. The reaction mixture was heated to $100{ }^{\circ} \mathrm{C}$ (aluminum block) for $16 \mathrm{~h}$. After the reaction was complete, the autoclave was cooled down with ice water to room temperature and the pressure 
was released carefully. After cooling to room temperature, the reaction mixture was directly purified by column chromatography on silica gel using petroleum and ethyl acetate to afford the corresponding imide product. 


\section{Spectroscopic Data of Products}<smiles>CCC(=O)N(C(=O)CC)c1ccccc1</smiles>

$N$-phenyl- $N$-propionylpropionamide (4) ${ }^{1}$ : Colorless oil, $81 \mathrm{mg}, 99 \%$ yield, $\mathrm{R}_{\mathrm{f}}=0.3(\mathrm{PE} / \mathrm{EtOAc}$ 10/1). ${ }^{\mathbf{1}} \mathbf{H}$ NMR (400 MHz, $\left.\mathrm{CDCl}_{3}\right) \delta 7.48-7.37(\mathrm{~m}, 3 \mathrm{H}), 7.13(\mathrm{~d}, \mathrm{~J}=7.8 \mathrm{~Hz}, 2 \mathrm{H}), 2.58(\mathrm{q}, \mathrm{J}=7.3$ $\mathrm{Hz}, 4 \mathrm{H}), 1.10$ (t, J = 7.3 Hz, 6H). ${ }^{13} \mathbf{C} \mathbf{N M R}\left(100 \mathrm{MHz}, \mathrm{CDCl}_{3}\right) \delta 176.7,139.1,129.7,128.9,128.7$, $32.2,9.0$.<smiles>CCC(=O)N(C(=O)CC)c1ccc(C)cc1</smiles>

$\boldsymbol{N}$-propionyl- $\boldsymbol{N}$-(p-tolyl)propionamide (5): Colorless oil, $82 \mathrm{mg}, 94 \%$ yield, $\mathrm{R}_{\mathrm{f}}=0.3$ (PE/EtOAc 10/1). ${ }^{1} \mathbf{H}$ NMR $\left(400 \mathrm{MHz} \mathrm{CDCl}_{3}\right) \delta 7.25(\mathrm{~d}, J=8.0 \mathrm{~Hz}, 2 \mathrm{H}), 7.00(\mathrm{~d}, J=8.1 \mathrm{~Hz}, 2 \mathrm{H})$, $2.58(\mathrm{~d}, J=7.3 \mathrm{~Hz}, 4 \mathrm{H}), 2.39(\mathrm{~s}, 3 \mathrm{H}), 1.10(\mathrm{t}, J=7.3 \mathrm{~Hz}, 6 \mathrm{H}) .{ }^{13} \mathbf{C} \mathbf{N M R}\left(100 \mathrm{MHz}, \mathrm{CDCl}_{3}\right) \delta 176.8$, 138.6, 136.4, 130.4, 128.6, 32.1, 21.2, 9.0. HRMS calculated for $\mathrm{C}_{13} \mathrm{H}_{17} \mathrm{NO}_{2}[\mathrm{M}+\mathrm{Na}]^{+}: 242.1151$, found 242.1153 .<smiles>CCC(=O)N(C(=O)CC)c1ccc(CC)cc1</smiles>

$\boldsymbol{N}$-(4-ethylphenyl)- $\boldsymbol{N}$-propionylpropionamide (6): Colorless oil, $82 \mathrm{mg}, 86 \%$ yield, $\mathrm{R}_{\mathrm{f}}=0.3$ (PE/EtOAc 10/1). ${ }^{1} \mathbf{H}$ NMR (400 MHz, $\left.\mathrm{CDCl}_{3}\right) \delta 7.27(\mathrm{~d}, \mathrm{~J}=7.7 \mathrm{~Hz}, 2 \mathrm{H}), 7.02(\mathrm{~d}, \mathrm{~J}=7.7 \mathrm{~Hz}, 2 \mathrm{H})$, 2.69 (q, J = 7.6 Hz, 2H), $2.58(\mathrm{q}, \mathrm{J}=7.3 \mathrm{~Hz}, 4 \mathrm{H}), 1.26(\mathrm{t}, \mathrm{J}=7.6 \mathrm{~Hz}, 3 \mathrm{H}), 1.10(\mathrm{t}, \mathrm{J}=7.3 \mathrm{~Hz}, 6 \mathrm{H})$. ${ }^{13}$ C NMR $\left(100 \mathrm{MHz}_{\mathrm{CDCl}}\right) \delta 176.8,144.8,136.6,129.1,128.6,32.1,28.5,15.3$, 9.0. HRMS calculated for $\mathrm{C}_{14} \mathrm{H}_{19} \mathrm{NO}_{2}[\mathrm{M}+\mathrm{H}]^{+}: 234.1489$, found 234.1491 .

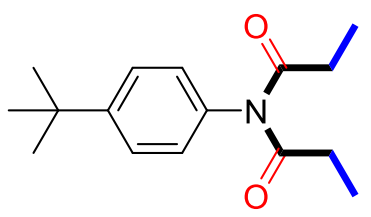

$N$-(4-(tert-butyl)phenyl)- $N$-propionylpropionamide (7): Colorless oil, $97 \mathrm{mg}, 93 \%$ yield, $\mathrm{R}_{\mathrm{f}}=$ 0.3 (PE/EtOAc 10/1). ${ }^{1} \mathbf{H}$ NMR $\left(400 \mathrm{MHz} \mathrm{CDCl}_{3}\right) \delta 7.37(\mathrm{~d}, \mathrm{~J}=8.1 \mathrm{~Hz}, 2 \mathrm{H}), 6.96(\mathrm{~d}, \mathrm{~J}=8.2 \mathrm{~Hz}$, $2 \mathrm{H}), 2.50(\mathrm{q}, \mathrm{J}=7.2 \mathrm{~Hz}, 4 \mathrm{H}), 1.26(\mathrm{~s}, 9 \mathrm{H}), 1.02(\mathrm{t}, \mathrm{J}=7.3 \mathrm{~Hz}, 6 \mathrm{H}) .{ }^{13} \mathbf{C} \mathbf{N M R}\left(100 \mathrm{MHz}, \mathrm{CDCl}_{3}\right) \delta$ $176.9,151.7,136.3,128.3,126.6,34.7,32.1,31.3,9.0$. HRMS calculated for $\mathrm{C}_{16} \mathrm{H}_{23} \mathrm{NO}_{2}[\mathrm{M}+\mathrm{H}]^{+}$: 262.1802, found 262.1799 .<smiles>CCC(=O)N(C(=O)CC)c1ccc(OC)cc1</smiles>

$\boldsymbol{N}$-(4-methoxy phenyl)- $\boldsymbol{N}$-propionylpropionamide (8): Colorless oil, $91 \mathrm{mg}, 97 \%$ yield, $\mathrm{R}_{\mathrm{f}}=0.2$ (PE/EtOAc 5/1). ${ }^{1} \mathbf{H}$ NMR $\left(400 \mathrm{MHz}_{\mathrm{CDCl}}\right) \delta 7.03(\mathrm{~d}, \mathrm{~J}=8.8 \mathrm{~Hz}, 2 \mathrm{H}), 6.95(\mathrm{~d}, \mathrm{~J}=8.9 \mathrm{~Hz}$, 2H), 3.83 (s, 3H), 2.58 (q, J = 7.3 Hz, 4H), 1.10 (t, J = 7.3 Hz, 6H). ${ }^{13} \mathbf{C ~ N M R}\left(100 \mathrm{MHz}, \mathrm{CDCl}_{3}\right)$ $\delta 177.0,159.5,131.6,129.8,114.9,55.5,32.1,9.0$. HRMS calculated for $\mathrm{C}_{13} \mathrm{H}_{17} \mathrm{NO}_{3}[\mathrm{M}+\mathrm{H}]^{+}$: 


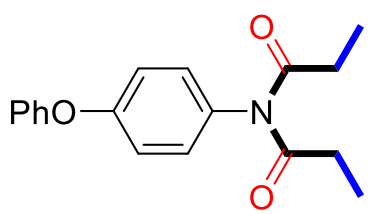

$N$-(4-phenoxyphenyl)- $N$-propionylpropionamide (9): Colorless oil, $109 \mathrm{mg}, 92 \%$ yield, $\mathrm{R}_{\mathrm{f}}=$ $0.2(\mathrm{PE} / \mathrm{EtOAc} 5 / 1) .{ }^{1} \mathbf{H}$ NMR $\left(400 \mathrm{MHz}, \mathrm{CDCl}_{3}\right) \delta 7.36(\mathrm{t}, \mathrm{J}=7.9 \mathrm{~Hz}, 2 \mathrm{H}), 7.15$ (t, J = $7.4 \mathrm{~Hz}$, 1H), $7.10-6.98(\mathrm{~m}, 6 \mathrm{H}), 2.60$ (q, J = 7.3 Hz, 4H), 1.11 (t, J = 7.3 Hz, 6H). ${ }^{13} \mathbf{C}$ NMR (100 MHz, $\left.\mathrm{CDCl}_{3}\right) \delta 176.8,157.8,156.2,133.6,130.2,130.0,124.1,119.7,119.1,32.2,9.0$. HRMS calculated for $\mathrm{C}_{18} \mathrm{H}_{19} \mathrm{NO}_{3}[\mathrm{M}+\mathrm{H}]^{+}:$298.1438, found 298.1436.<smiles>CCC(=O)N(C(=O)CC)c1ccc(OC(F)(F)F)cc1</smiles>

$N$-propionyl- $N$-(4-(trifluoromethoxy)phenyl)propionamide (10): White solid, $106 \mathrm{mg}, 92 \%$ yield, $\mathrm{R}_{\mathrm{f}}=0.2(\mathrm{PE} / \mathrm{EtOAc} 5 / 1) .{ }^{1} \mathbf{H}$ NMR $\left(400 \mathrm{MHz}, \mathrm{CDCl}_{3}\right) \delta 7.31(\mathrm{~d}, \mathrm{~J}=8.4 \mathrm{~Hz}, 2 \mathrm{H}), 7.18(\mathrm{~d}, \mathrm{~J}$ $=8.4 \mathrm{~Hz}, 2 \mathrm{H}), 2.58(\mathrm{q}, \mathrm{J}=7.2 \mathrm{~Hz}, 4 \mathrm{H}), 1.11(\mathrm{t}, \mathrm{J}=7.3 \mathrm{~Hz}, 6 \mathrm{H}) \cdot{ }^{13} \mathbf{C} \mathbf{N M R}\left(100 \mathrm{MHz}, \mathrm{CDCl}_{3}\right) \delta$ 176.4, $149.1(\mathrm{~d}, \mathrm{~J}=1.9 \mathrm{~Hz}), 137.5,130.6,122.0,120.3(\mathrm{q}, \mathrm{J}=258.0 \mathrm{~Hz}), 32.2,8.8 .{ }^{19} \mathbf{F}$ NMR $(56$ $\left.\mathrm{MHz}_{2} \mathrm{CDCl}_{3}\right) \delta$-62.69. HRMS calculated for $\mathrm{C}_{13} \mathrm{H}_{14} \mathrm{~F}_{3} \mathrm{NO}_{3}[\mathrm{M}+\mathrm{H}]^{+}: 290.0999$, found 290.0995 .<smiles>CCC(=O)N(C(=O)CC)c1ccc(S(C)(=O)=O)cc1</smiles>

$\boldsymbol{N}$-(4-(methylthio)phenyl)- $\boldsymbol{N}$-propionylpropionamide (11): Colorless oil, $89 \mathrm{mg}, 89 \%$ yield, $\mathrm{R}_{\mathrm{f}}$ $=0.3(\mathrm{PE} / \mathrm{EtOAc} 5 / 1) .{ }^{1} \mathbf{H}$ NMR $\left(400 \mathrm{MHz}, \mathrm{CDCl}_{3}\right) \delta 7.30(\mathrm{~d}, \mathrm{~J}=8.7 \mathrm{~Hz}, 2 \mathrm{H}), 7.03(\mathrm{~d}, \mathrm{~J}=8.6 \mathrm{~Hz}$, 2H), $2.58(\mathrm{q}, \mathrm{J}=7.3 \mathrm{~Hz}, 4 \mathrm{H}), 2.50$ (s, 3H), 1.10 (t, J = 7.3 Hz, 6H). ${ }^{13} \mathbf{C ~ N M R ~}\left(100 \mathrm{MHz}, \mathrm{CDCl}_{3}\right) \delta$ 176.7, 139.7, 135.8, 129.2, 127.2, 32.1, 15.5, 9.0. HRMS calculated for $\mathrm{C}_{13} \mathrm{H}_{17} \mathrm{NO}_{2} \mathrm{~S}[\mathrm{M}+\mathrm{Na}]^{+}$: 274.0872 , found 274.0860 .<smiles>CCC(=O)N(C(=O)CC)c1ccc(F)cc1</smiles>

$N$-(4-fluorophenyl)- $\mathrm{N}$-propionylpropionamide (12): Colorless oil, $86 \mathrm{mg}, 96 \%$ yield, $\mathrm{R}_{\mathrm{f}}=0.2$ (PE/EtOAc 10/1). ${ }^{1} \mathbf{H}$ NMR (400 MHz, $\left.\mathrm{CDCl}_{3}\right) \delta 7.21-7.07$ (m, 4H), 2.58 (q, J = 7.2 Hz, 4H), $1.11(\mathrm{t}, \mathrm{J}=7.2 \mathrm{~Hz}, 6 \mathrm{H}) .{ }^{13} \mathbf{C}$ NMR $\left(100 \mathrm{MHz}, \mathrm{CDCl}_{3}\right) \delta$ 176.6, $162.4(\mathrm{~d}, \mathrm{~J}=248.7 \mathrm{~Hz}), 135.0(\mathrm{~d}, \mathrm{~J}=$ $3.5 \mathrm{~Hz}), 130.7(\mathrm{~d}, \mathrm{~J}=8.7 \mathrm{~Hz}), 116.7(\mathrm{~d}, \mathrm{~J}=22.9 \mathrm{~Hz}), 32.2,8.9 .{ }^{19} \mathbf{F} \mathbf{N M R}\left(56 \mathrm{MHz}, \mathrm{CDCl}_{3}\right) \delta-117.60$. HRMS calculated for $\mathrm{C}_{12} \mathrm{H}_{14} \mathrm{FNO}_{2}[\mathrm{M}+\mathrm{H}]^{+}:$224.1081, found 224.1075 .<smiles>CCC(=O)N(C(=O)CC)c1ccc(Cl)cc1</smiles>

$\mathrm{N}$-(4-chlorophenyl)- $\mathrm{N}$-propionylpropionamide (13): Colorless oil, $94 \mathrm{mg}, 98 \%$ yield, $\mathrm{R}_{\mathrm{f}}=$ 0.2 (PE/EtOAc 10/1). ${ }^{1} \mathbf{H}$ NMR $\left(400 \mathrm{MHz}, \mathrm{CDCl}_{3}\right) \delta 7.43(\mathrm{~d}, J=8.5 \mathrm{~Hz}, 2 \mathrm{H}), 7.07$ (d, $J=8.5 \mathrm{~Hz}$, 2H), 2.57 (q, $J=7.3 \mathrm{~Hz}, 4 \mathrm{H}), 1.10$ (t, $J=7.3 \mathrm{~Hz}, 6 \mathrm{H}) .{ }^{13} \mathbf{C ~ N M R}\left(100 \mathrm{MHz}, \mathrm{CDCl}_{3}\right) \delta 176.4,137.6$, 
134.7, 130.3, 127.0, 32.2, 8.9. HRMS calculated for $\mathrm{C}_{12} \mathrm{H}_{14} \mathrm{ClNO}_{2}[\mathrm{M}+\mathrm{H}]^{+}: 240.0786$, found 240.0786 .<smiles>CCC(=O)N(C(=O)CC)c1ccc(Br)cc1</smiles>

$\boldsymbol{N}$-(4-bromophenyl)- $\boldsymbol{N}$-propionylpropionamide (14): Colorless oil, $104 \mathrm{mg}, 92 \%$ yield, $\mathrm{R}_{\mathrm{f}}=$ 0.2 (PE/EtOAc 10/1). ${ }^{1} \mathbf{H}$ NMR $\left(400 \mathrm{MHz}, \mathrm{CDCl}_{3}\right) \delta 7.59(\mathrm{~d}, J=8.2 \mathrm{~Hz}, 2 \mathrm{H}), 7.01(\mathrm{~d}, J=8.2 \mathrm{~Hz}$, $2 \mathrm{H}), 2.57$ (q, $J=7.3 \mathrm{~Hz}, 4 \mathrm{H}), 1.10$ (t, $J=7.3 \mathrm{~Hz}, 6 \mathrm{H}) .{ }^{13} \mathbf{C}$ NMR $\left(100 \mathrm{MHz}, \mathrm{CDCl}_{3}\right) \delta 176.3,138.1$, 133.0, 130.7, 122.8, 32.2, 8.9. HRMS calculated for $\mathrm{C}_{12} \mathrm{H}_{14} \mathrm{BrNO}_{2}[\mathrm{M}+\mathrm{H}]^{+}: 284.0281$, found 284.0276 .<smiles>CCC(=O)N(C(=O)CC)c1ccc(I)cc1</smiles>

$N$-(4-iodophenyl)- $N$-propionylpropionamide (15): Colorless oil, $81 \mathrm{mg}, 61 \%$ yield, $\mathrm{R}_{\mathrm{f}}=0.2$ (PE/EtOAc 10/1). ${ }^{1} \mathbf{H}$ NMR (400 MHz, $\left.\mathrm{CDCl}_{3}\right) \delta 7.79(\mathrm{~d}, \mathrm{~J}=8.2 \mathrm{~Hz}, 2 \mathrm{H}), 6.88(\mathrm{~d}, \mathrm{~J}=8.1 \mathrm{~Hz}, 2 \mathrm{H})$, 2.57 (q, J = 7.3 Hz, 4H), $1.10(\mathrm{t}, \mathrm{J}=7.3 \mathrm{~Hz}, 6 \mathrm{H}) .{ }^{13} \mathbf{C} \mathbf{N M R}\left(100 \mathrm{MHz}, \mathrm{CDCl}_{3}\right) \delta 176.3,139.0$, 138.9, 130.9, 94.4, 32.2, 8.9. HRMS calculated for $\mathrm{C}_{12} \mathrm{H}_{14} \mathrm{INO}_{2}[\mathrm{M}+\mathrm{Na}]^{+}: 353.9961$, found 353.9955 .<smiles>CCC(=O)N(C(=O)CC)c1ccc(N(C)C)cc1</smiles>

$\mathrm{N}$-(4-(dimethylamino)phenyl)- $\mathrm{N}$-propionylpropionamide (16): White solid, $61 \mathrm{mg}, 61 \%$ yield, $\mathrm{R}_{\mathrm{f}}=0.2(\mathrm{PE} / \mathrm{EtOAc} 5 / 1) .{ }^{1} \mathbf{H}$ NMR $\left(400 \mathrm{MHz}, \mathrm{CDCl}_{3}\right) \delta 6.94(\mathrm{~d}, J=8.5 \mathrm{~Hz}, 2 \mathrm{H}), 6.72(\mathrm{~d}, J=8.5$ $\mathrm{Hz}, 2 \mathrm{H}), 2.98$ (s, 6H), 2.59 (q, $J=7.3 \mathrm{~Hz}, 4 \mathrm{H}), 1.10$ (t, $J=7.3 \mathrm{~Hz}, 6 \mathrm{H}) .{ }^{13} \mathbf{C}$ NMR $(100 \mathrm{MHz}$, $\left.\mathrm{CDCl}_{3}\right) \delta 177.3,150.3,129.2,127.4,112.7,40.4,32.1,9.1$. HRMS calculated for $\mathrm{C}_{14} \mathrm{H}_{20} \mathrm{~N}_{2} \mathrm{O}_{2}$ $[\mathrm{M}+\mathrm{H}]^{+}: 249.1598$, found 249.1599 .<smiles>CCC(=O)N(C(=O)CC)c1ccc(C(F)(F)F)cc1</smiles>

$N$-propionyl- $N$-(4-(trifluoromethyl)phenyl)propionamide (17): Colorless oil, $95 \mathrm{mg}, 87 \%$ yield, $\mathrm{R}_{\mathrm{f}}=0.4(\mathrm{PE} / \mathrm{EtOAc} 10 / 1) .{ }^{1} \mathbf{H}$ NMR $\left(400 \mathrm{MHz}, \mathrm{CDCl}_{3}\right) \delta 7.73(\mathrm{~d}, \mathrm{~J}=8.0 \mathrm{~Hz}, 2 \mathrm{H}), 7.28(\mathrm{~d}$, $\mathrm{J}=7.9 \mathrm{~Hz}, 2 \mathrm{H}), 2.58(\mathrm{q}, \mathrm{J}=7.2 \mathrm{~Hz}, 4 \mathrm{H}), 1.12(\mathrm{t}, \mathrm{J}=7.2 \mathrm{~Hz}, 6 \mathrm{H}) .{ }^{13} \mathbf{C} \mathbf{N M R}\left(100 \mathrm{MHz}, \mathrm{CDCl}_{3}\right) \delta$ 176.2, 142.4, 131.0 (q, J = 32.8 Hz), 129.6, 126.9 (q, J = 3.8 Hz), 123.7 (q, J = 272.4 Hz), 32.2, 8.8. ${ }^{19} \mathbf{F}$ NMR $\left(56 \mathrm{MHz}, \mathrm{CDCl}_{3}\right) \delta$-68.06. HRMS calculated for $\mathrm{C}_{13} \mathrm{H}_{14} \mathrm{~F}_{3} \mathrm{NO}_{2}[\mathrm{M}+\mathrm{Na}]^{+}: 296.0869$, found 296.0869 .<smiles>CCC(=O)N(C(=O)CC)c1ccc(C=O)cc1</smiles>

$N$-(4-formylphenyl)- $N$-propionylpropionamide (18): Yellow solid, $61 \mathrm{mg}, 65 \%$ yield, $\mathrm{R}_{\mathrm{f}}=0.2$ 
(PE/EtOAc 5/1). ${ }^{1} \mathbf{H}$ NMR (400 MHz, $\left.\mathrm{CDCl}_{3}\right) \delta 10.07$ (s, 1H), 7.99 (d, J = 7.9 Hz, 2H), 7.34 (d, J = $7.9 \mathrm{~Hz}, 2 \mathrm{H}), 2.59$ (q, J = 7.2 Hz, 4H), $1.12(\mathrm{t}, \mathrm{J}=7.2 \mathrm{~Hz}, 6 \mathrm{H}) .{ }^{13} \mathbf{C} \mathbf{~ N M R}\left(100 \mathrm{MHz}, \mathrm{CDCl}_{3}\right) \delta 191.0$, 176.1, 144.5, 136.3, 130.9, 130.0, 32.2, 8.9. HRMS calculated for $\mathrm{C}_{13} \mathrm{H}_{15} \mathrm{NO}_{3}[\mathrm{M}+\mathrm{H}]^{+}: 234.1125$, found 234.1122 .<smiles>CCOC(=O)c1ccc(N(C(=O)CC)C(=O)CC)cc1</smiles>

$N$-(4-(ethylperoxy)phenyl)- $N$-propionylpropionamide (19): White solid, $104 \mathrm{mg}, 98 \%$ yield, $\mathrm{R}_{\mathrm{f}}=0.3(\mathrm{PE} / \mathrm{EtOAc} 10 / 1) .{ }^{1} \mathbf{H}$ NMR $\left(400 \mathrm{MHz}, \mathrm{CDCl}_{3}\right) \delta 8.15(\mathrm{~d}, \mathrm{~J}=8.1 \mathrm{~Hz}, 2 \mathrm{H}), 7.23(\mathrm{~d}, \mathrm{~J}=8.1$ $\mathrm{Hz}, 2 \mathrm{H}), 4.40(\mathrm{q}, \mathrm{J}=7.1 \mathrm{~Hz}, 2 \mathrm{H}), 2.58(\mathrm{q}, \mathrm{J}=7.3 \mathrm{~Hz}, 4 \mathrm{H}), 1.40(\mathrm{t}, \mathrm{J}=7.1 \mathrm{~Hz}, 3 \mathrm{H}), 1.11$ (t, J = 7.3 Hz, 6H). ${ }^{13} \mathbf{C}$ NMR $\left(100 \mathrm{MHz}, \mathrm{CDCl}_{3}\right) \delta 176.2,165.6,143.2,131.0,130.9,129.1,61.3,32.2,14.3,8.9$. HRMS calculated for $\mathrm{C}_{15} \mathrm{H}_{19} \mathrm{NO}_{4}[\mathrm{M}+\mathrm{H}]^{+}: 278.1387$, found 278.1394 .<smiles>CCC(=O)N(C(=O)CC)c1ccc(C(C)=O)cc1</smiles>

$N$-(4-acetylphenyl)- $N$-propionylpropionamide (20): White solid, $83 \mathrm{mg}, 84 \%$ yield, $\mathrm{R}_{\mathrm{f}}=0.2$ (PE/EtOAc 5/1). ${ }^{1} \mathbf{H}$ NMR (400 MHz, $\left.\mathrm{CDCl}_{3}\right) \delta 8.05(\mathrm{~d}, \mathrm{~J}=8.2 \mathrm{~Hz}, 2 \mathrm{H}), 7.26(\mathrm{~d}, \mathrm{~J}=8.2 \mathrm{~Hz}, 2 \mathrm{H})$, $2.63(\mathrm{~s}, 3 \mathrm{H}), 2.58(\mathrm{q}, \mathrm{J}=7.3 \mathrm{~Hz}, 4 \mathrm{H}), 1.11(\mathrm{t}, \mathrm{J}=7.3 \mathrm{~Hz}, 6 \mathrm{H}) .{ }^{13} \mathbf{C} \mathbf{~ N M R}\left(100 \mathrm{MHz}, \mathrm{CDCl}_{3}\right) \delta 196.9$, $176.2,143.4,137.1,129.7,129.4,32.2,26.7,8.7$. HRMS calculated for $\mathrm{C}_{14} \mathrm{H}_{17} \mathrm{NO}_{3}[\mathrm{M}+\mathrm{H}]^{+}$: 248.1281 , found 248.1286 .

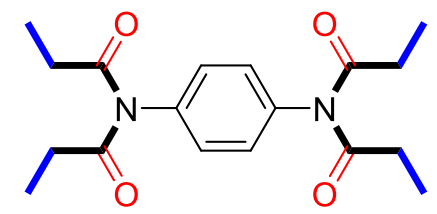

$N, N^{\prime}$-(1,4-phenylene)bis( $N$-propionylpropionamide) (21): Colorless oil, $83 \mathrm{mg}, 63 \%$ yield, $\mathrm{R}_{\mathrm{f}}$ $=0.3(\mathrm{PE} / \mathrm{EtOAc} \mathrm{10/1)}){ }^{1} \mathbf{H}$ NMR $\left(400 \mathrm{MHz}, \mathrm{CDCl}_{3}\right) \delta 7.24(\mathrm{~s}, 4 \mathrm{H}), 2.63(\mathrm{q}, \mathrm{J}=7.2 \mathrm{~Hz}, 8 \mathrm{H}), 1.12$ $(\mathrm{t}, \mathrm{J}=7.3 \mathrm{~Hz}, 12 \mathrm{H}) .{ }^{13} \mathbf{C}$ NMR $\left(100 \mathrm{MHz}, \mathrm{CDCl}_{3}\right) \delta 176.4,139.4,130.3,32.2,8.8$. HRMS calculated for $\mathrm{C}_{18} \mathrm{H}_{24} \mathrm{~N}_{2} \mathrm{O}_{4}[\mathrm{M}+\mathrm{H}]^{+}: 333.1809$, found 333.1813 .

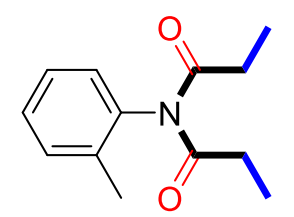

$N$-propionyl- $N$-(o-tolyl)propionamide (22): Colorless oil, $86 \mathrm{mg}, 98 \%$ yield, $\mathrm{R}_{\mathrm{f}}=0.2$ (PE/EtOAc 10/1). ${ }^{1} \mathbf{H}$ NMR $\left(400 \mathrm{MHz}, \mathrm{CDCl}_{3}\right) \delta 7.34-7.24(\mathrm{~m}, 3 \mathrm{H}), 7.04(\mathrm{~d}, \mathrm{~J}=7.4 \mathrm{~Hz}, 1 \mathrm{H})$, $2.69-2.41(\mathrm{~m}, 4 \mathrm{H}), 2.12(\mathrm{~s}, 3 \mathrm{H}), 1.11(\mathrm{t}, \mathrm{J}=7.3 \mathrm{~Hz}, 6 \mathrm{H}) .{ }^{13} \mathbf{C} \mathbf{N M R}\left(100 \mathrm{MHz}, \mathrm{CDCl}_{3}\right) \delta 176.4$, $138.1,135.9,131.3,129.0,128.9,127.4,31.8,17.5,8.9$. HRMS calculated for $\mathrm{C}_{13} \mathrm{H}_{17} \mathrm{NO}_{2}[\mathrm{M}+\mathrm{H}]^{+}$: 220.1332 , found 220.1337 . 
<smiles>CCC(=O)N(C(=O)CC)c1ccccc1Br</smiles>

$N$-(2-isopropylphenyl)- $N$-propionylpropionamide (23): Colorless oil, $98 \mathrm{mg}, 99 \%$ yield, $\mathrm{R}_{\mathrm{f}}=$ 0.3 (PE/EtOAc 10/1). ${ }^{1} \mathbf{H}$ NMR $\left(400 \mathrm{MHz}, \mathrm{CDCl}_{3}\right) \delta 7.46-7.35(\mathrm{~m}, 2 \mathrm{H}), 7.29-7.20(\mathrm{~m}, 1 \mathrm{H})$, $7.01(\mathrm{~d}, \mathrm{~J}=7.8 \mathrm{~Hz}, 1 \mathrm{H}), 2.79$ (hept, J = 6.9 Hz, 1H), $2.69-2.56(\mathrm{~m}, 2 \mathrm{H}), 2.56-2.43(\mathrm{~m}, 2 \mathrm{H}), 1.17$ (d, $\mathrm{J}=6.9 \mathrm{~Hz}, 6 \mathrm{H}), 1.11(\mathrm{t}, \mathrm{J}=7.3 \mathrm{~Hz}, 6 \mathrm{H}) .{ }^{13} \mathbf{C}$ NMR $\left(100 \mathrm{MHz}, \mathrm{CDCl}_{3}\right) \delta 176.7,146.2,136.5,129.4$, $128.9,127.1,127.0,32.0,28.0,23.6,9.0$. HRMS calculated for $\mathrm{C}_{15} \mathrm{H}_{21} \mathrm{NO}_{2}[\mathrm{M}+\mathrm{H}]^{+}:$: 248.1645, found 248.1652 .<smiles>CCC(=O)N(C(=O)CC)c1ccccc1I</smiles>

$N$-(2-iodophenyl)- $N$-propionylpropionamide (24): Colorless oil, $104 \mathrm{mg}, 79 \%$ yield, $\mathrm{R}_{\mathrm{f}}=0.2$ (PE/EtOAc 10/1). ${ }^{1} \mathbf{H}$ NMR (400 MHz, $\left.\mathrm{CDCl}_{3}\right) \delta 7.93(\mathrm{~d}, \mathrm{~J}=7.8 \mathrm{~Hz}, 1 \mathrm{H}), 7.45(\mathrm{t}, \mathrm{J}=7.6 \mathrm{~Hz}, 1 \mathrm{H})$, $7.23(\mathrm{~d}, \mathrm{~J}=7.8 \mathrm{~Hz}, 1 \mathrm{H}), 7.12(\mathrm{t}, \mathrm{J}=7.7 \mathrm{~Hz}, 1 \mathrm{H}), 2.56(\mathrm{q}, \mathrm{J}=7.2 \mathrm{~Hz}, 4 \mathrm{H}), 1.13(\mathrm{t}, \mathrm{J}=7.2 \mathrm{~Hz}, 6 \mathrm{H})$. ${ }^{13}$ C NMR $\left(100 \mathrm{MHz}, \mathrm{CDCl}_{3}\right) \delta 175.8,141.9,140.0,130.3,130.1,129.8,100.9,32.2,8.8$. HRMS calculated for $\mathrm{C}_{12} \mathrm{H}_{14} \mathrm{INO}_{2}[\mathrm{M}+\mathrm{H}]^{+}: 332.0142$, found 332.0134 .<smiles>CCC(=O)N(C(=O)CC)c1c(C)cccc1C</smiles>

$N$-(2,6-dimethylphenyl)- $N$-propionylpropionamide (25): White solid, $92 \mathrm{mg}, 99 \%$ yield, $\mathrm{R}_{\mathrm{f}}=$ 0.2 (PE/EtOAc 10/1). ${ }^{1} \mathbf{H}$ NMR (400 MHz, $\left.\mathrm{CDCl}_{3}\right) \delta 7.23-7.17(\mathrm{~m}, 1 \mathrm{H}), 7.13(\mathrm{~d}, \mathrm{~J}=7.4 \mathrm{~Hz}, 2 \mathrm{H})$, $2.52(\mathrm{q}, \mathrm{J}=7.3 \mathrm{~Hz}, 4 \mathrm{H}), 2.10(\mathrm{~s}, 6 \mathrm{H}), 1.12(\mathrm{t}, \mathrm{J}=7.3 \mathrm{~Hz}, 6 \mathrm{H}) .{ }^{13} \mathbf{C} \mathbf{~ N M R}\left(100 \mathrm{MHz}, \mathrm{CDCl}_{3}\right) \delta 176.2$, 137.2, 135.7, 128.9, 128.7, 31.3, 17.8, 9.0. HRMS calculated for $\mathrm{C}_{14} \mathrm{H}_{19} \mathrm{NO}_{2}[\mathrm{M}+\mathrm{H}]^{+}:$: 234.1489, found 234.1491 .<smiles>CCCC(=O)N(C(=O)CC)c1c(Br)cccc1CC</smiles>

$\mathrm{N}$-(2,6-diisopropylphenyl)- $\mathrm{N}$-propionylpropionamide (26): Colorless oil, $109 \mathrm{mg}, 94 \%$ yield, $\mathrm{R}_{\mathrm{f}}=0.3\left(\mathrm{PE} / \mathrm{EtOAc}\right.$ 10/1). ${ }^{1} \mathbf{H}$ NMR $\left(400 \mathrm{MHz}, \mathrm{CDCl}_{3}\right) \delta 7.39(\mathrm{t}, \mathrm{J}=7.7 \mathrm{~Hz}, 1 \mathrm{H}), 7.23(\mathrm{~d}, \mathrm{~J}=7.7$ $\mathrm{Hz}, 2 \mathrm{H}), 2.77$ (hept, $\mathrm{J}=6.9 \mathrm{~Hz}, 2 \mathrm{H}), 2.54(\mathrm{q}, \mathrm{J}=7.3 \mathrm{~Hz}, 4 \mathrm{H}), 1.15(\mathrm{~d}, \mathrm{~J}=7.3 \mathrm{~Hz}, 12 \mathrm{H}), 1.12(\mathrm{t}, \mathrm{J}=$ $7.5 \mathrm{~Hz}, 6 \mathrm{H}) .{ }^{13} \mathbf{C} \mathbf{N M R}\left(100 \mathrm{MHz}, \mathrm{CDCl}_{3}\right) \delta 176.9,145.8,134.1,129.5,124.4,31.6,28.5,24.0,9.0$. HRMS calculated for $\mathrm{C}_{18} \mathrm{H}_{27} \mathrm{NO}_{2}[\mathrm{M}+\mathrm{H}]^{+}: 290.2115$, found 290.2117 .<smiles>CCC(=O)N(C(=O)CC)c1cccc(C)c1</smiles>

$N$-propionyl- $N$-(m-tolyl)propionamide (27): Colorless oil, $83 \mathrm{mg}, 95 \%$ yield, $\mathrm{R}_{\mathrm{f}}=0.2$ 
(PE/EtOAc 10/1). ${ }^{1} \mathbf{H}$ NMR (400 MHz, $\left.\mathrm{CDCl}_{3}\right) \delta 7.33(\mathrm{t}, J=7.6 \mathrm{~Hz}, 1 \mathrm{H}), 7.21(\mathrm{~d}, J=7.7 \mathrm{~Hz}, 1 \mathrm{H})$, $6.97-6.88$ (m, 2H), 2.58 (q, $J=7.3 \mathrm{~Hz}, 4 \mathrm{H}), 2.37$ (s, 3H), 1.10 (t, $J=7.3 \mathrm{~Hz}, 6 \mathrm{H}) .{ }^{13}$ C NMR $(100$ $\left.\mathrm{MHz}_{2} \mathrm{CDCl}_{3}\right) \delta 176.7,139.8,139.08,129.5,129.5,125.9,32.1,21.3,9.0$. HRMS calculated for $\mathrm{C}_{13} \mathrm{H}_{17} \mathrm{NO}_{2}[\mathrm{M}+\mathrm{H}]^{+}: 220.1332$, found 220.1335 .

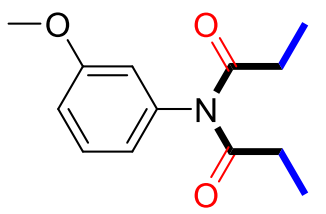

$N$-(3-methoxyphenyl)- $N$-propionylpropionamide (28): Colorless oil, $93 \mathrm{mg}, 99 \%$ yield, $\mathrm{R}_{\mathrm{f}}=$ 0.2 (PE/EtOAc 5/1). ${ }^{1} \mathbf{H}$ NMR (400 MHz, $\left.\mathrm{CDCl}_{3}\right) \delta 7.35$ (t, J = $\left.8.1 \mathrm{~Hz}, 1 \mathrm{H}\right), 6.94$ (d, J = $8.2 \mathrm{~Hz}$, $1 \mathrm{H}), 6.72(\mathrm{~d}, \mathrm{~J}=7.8 \mathrm{~Hz}, 1 \mathrm{H}), 6.67(\mathrm{~s}, 1 \mathrm{H}), 3.80(\mathrm{~s}, 3 \mathrm{H}), 2.60(\mathrm{q}, \mathrm{J}=7.3 \mathrm{~Hz}, 4 \mathrm{H}), 1.10(\mathrm{t}, \mathrm{J}=7.3 \mathrm{~Hz}$, 6H). ${ }^{13} \mathrm{C} \mathrm{NMR}\left(100 \mathrm{MHz}, \mathrm{CDCl}_{3}\right) \delta 176.6,160.6,140.2,130.3,121.1,114.7,114.3,55.4(\mathrm{~d}, \mathrm{~J}=1.8$ $\mathrm{Hz}$ ), 32.0, 8.9. HRMS calculated for $\mathrm{C}_{13} \mathrm{H}_{17} \mathrm{NO}_{3}[\mathrm{M}+\mathrm{H}]^{+}: 236.1281$, found 236.1279 .<smiles>CCC(=O)N(C(=O)CC)c1cccc(Br)c1</smiles>

$N$-(3-bromophenyl)- $N$-propionylpropionamide (29): White so lid, $104 \mathrm{mg}, 92 \%$ yield, $\mathrm{R}_{\mathrm{f}}=0.2$ (PE/EtOAc 10/1). ${ }^{1} \mathbf{H}$ NMR (400 MHz, $\left.\mathrm{CDCl}_{3}\right) \delta 7.55(\mathrm{~d}, \mathrm{~J}=8.0 \mathrm{~Hz}, 1 \mathrm{H}), 7.37-7.29(\mathrm{~m}, 2 \mathrm{H})$, 7.09 (d, J = 7.9 Hz, 1H), 2.58 (q, J = 7.2 Hz, 4H), 1.11 (t, J = 7.3 Hz, 6H). ${ }^{13} \mathbf{C}$ NMR (100 MHz, $\left.\mathrm{CDCl}_{3}\right) \delta 176.3,140.3,132.2,131.9,130.9,127.8,123.0,32.2,8.9$. HRMS calculated for $\mathrm{C}_{12} \mathrm{H}_{14} \mathrm{BrNO}_{2}[\mathrm{M}+\mathrm{Na}]^{+}: 306.0100$, found 306.0088 .<smiles>CCC(=O)N(C(=O)CC)c1cccc(N(C)C)c1</smiles>

$N$-(3-(dimethylamino)phenyl)- $N$-propionylpropionamide (30): White solid, $75 \mathrm{mg}, 76 \%$ yield, $\mathrm{R}_{\mathrm{f}}=0.2(\mathrm{PE} / \mathrm{EtOAc} 5 / 1) .{ }^{1} \mathbf{H}$ NMR $\left(400 \mathrm{MHz}, \mathrm{CDCl}_{3}\right) \delta 7.27(\mathrm{t}, \mathrm{J}=8.0 \mathrm{~Hz}, 1 \mathrm{H}), 6.73(\mathrm{~d}, \mathrm{~J}=8.4$ $\mathrm{Hz}, 1 \mathrm{H}), 6.45(\mathrm{~d}, \mathrm{~J}=7.7 \mathrm{~Hz}, 1 \mathrm{H}), 6.40(\mathrm{~s}, 1 \mathrm{H}), 2.95$ (s, 6H), 2.63 (q, J = 7.3 Hz, 4H), 1.11 (t, J = 7.3 $\mathrm{Hz}, 6 \mathrm{H}) .{ }^{13} \mathrm{C}$ NMR $\left(100 \mathrm{MHz}, \mathrm{CDCl}_{3}\right) \delta 176.8,151.5,140.0,130.0,116.3,112.4,40.4,31.9,9.1$. HRMS calculated for $\mathrm{C}_{14} \mathrm{H}_{20} \mathrm{~N}_{2} \mathrm{O}_{2}[\mathrm{M}+\mathrm{Na}]^{+}: 271.1417$, found 271.1426 .<smiles>CCC(=O)N(C(=O)CC)c1cccc(C#N)c1</smiles>

$N$-(3-cyanophenyl)- $N$-propionylpropionamide (31): Colorless oil, $81 \mathrm{mg}, 88 \%$ yield, $\mathrm{R}_{\mathrm{f}}=0.2$ (PE/EtOAc 5/1). ${ }^{1} \mathbf{H}$ NMR (400 MHz, $\left.\mathrm{CDCl}_{3}\right) \delta 7.73(\mathrm{~d}, \mathrm{~J}=7.7 \mathrm{~Hz}, 1 \mathrm{H}), 7.61$ (t, J = 7.9 Hz, 1H), 7.49 (s, 1H), $7.42(\mathrm{~d}, \mathrm{~J}=8.0 \mathrm{~Hz}, 1 \mathrm{H}), 2.57$ (q, J = 7.3 Hz, 4H), 1.12 (t, J = 7.3 Hz, 6H). ${ }^{13} \mathbf{C}$ NMR $\left(100 \mathrm{MHz}, \mathrm{CDCl}_{3}\right) \delta 176.0,140.1,133.9,132.8,132.4,130.7,117.5,114.0,32.3,8.8$. HRMS calculated for $\mathrm{C}_{13} \mathrm{H}_{14} \mathrm{~N}_{2} \mathrm{O}_{2}[\mathrm{M}+\mathrm{H}]^{+}: 231.1128$, found 231.1122 . 


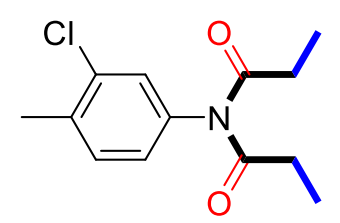

$\boldsymbol{N}$-(3-chloro-4-methylphenyl)- $\boldsymbol{N}$-propionylpropionamide (32): Colorless oil, $101 \mathrm{mg}, 99 \%$ yield, $\mathrm{R}_{\mathrm{f}}=0.2\left(\mathrm{PE} /\right.$ EtOAc 10/1). ${ }^{\mathbf{1}} \mathbf{H} \mathbf{N M R}\left(400 \mathrm{MHz}, \mathrm{CDCl}_{3}\right) \delta 7.31(\mathrm{~d}, \mathrm{~J}=8.0 \mathrm{~Hz}, 1 \mathrm{H}), 7.15(\mathrm{~s}$, 1H), $6.94(\mathrm{~d}, \mathrm{~J}=7.9 \mathrm{~Hz}, 1 \mathrm{H}), 2.59$ (q, J = $7.3 \mathrm{~Hz}, 4 \mathrm{H}), 2.41(\mathrm{~s}, 3 \mathrm{H}), 1.10(\mathrm{t}, \mathrm{J}=7.3 \mathrm{~Hz}, 6 \mathrm{H}) .{ }^{13} \mathrm{C}$ NMR $\left(100 \mathrm{MHz} \mathrm{CDCl}_{3}\right) \delta 176.4,137.7,136.9,135.1,131.7,129.5,127.2,32.1,19.8,8.9$. HRMS calculated for $\mathrm{C}_{13} \mathrm{H}_{16} \mathrm{ClNO}_{2}[\mathrm{M}+\mathrm{H}]^{+}:$254.0942, found 254.0936.

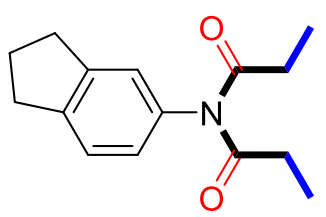

$\boldsymbol{N}$-(2,3-dihydro-1H-inden-5-yl)- $\boldsymbol{N}$-propionylpropionamide (33): White solid, $62 \mathrm{mg}, 63 \%$ yield, $\mathrm{R}_{\mathrm{f}}=0.2(\mathrm{PE} / \mathrm{EtOAc} 10 / 1) .{ }^{\mathbf{1}} \mathbf{H} \mathbf{N M R}\left(400 \mathrm{MHz}_{\mathrm{CDCl}}\right) \delta 7.27(\mathrm{~d}, \mathrm{~J}=7.7 \mathrm{~Hz}, 1 \mathrm{H}), 6.96(\mathrm{~s}$, $1 \mathrm{H}), 6.86(\mathrm{~d}, \mathrm{~J}=7.8 \mathrm{~Hz}, 1 \mathrm{H}), 2.93$ (t, J = 7.5 Hz, 4H), 2.59 (q, J = 7.3 Hz, 4H), 2.12 (p, J = 7.4 Hz, $2 \mathrm{H}), 1.10(\mathrm{t}, \mathrm{J}=7.3 \mathrm{~Hz}, 6 \mathrm{H}) .{ }^{13} \mathrm{C}$ NMR (100 MHz, $\left.\mathrm{CDCl}_{3}\right) \delta 177.0,146.0,144.9,137.1,126.5$, 125.3, 124.7, 32.8, 32.6, 32.1, 25.6, 9.0. HRMS calculated for $\mathrm{C}_{15} \mathrm{H}_{19} \mathrm{NO}_{2}[\mathrm{M}+\mathrm{H}]^{+}: 246.1489$, found 246.1486 .

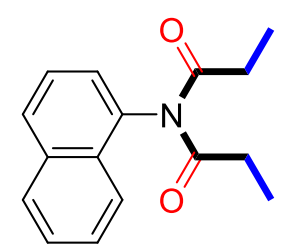

$\boldsymbol{N}$-(naphthalen-1-yl)- $\boldsymbol{N}$-propionylpropionamide (34): Colorless oil, $99 \mathrm{mg}, 97 \%$ yield, $\mathrm{R}_{\mathrm{f}}=0.2$ (PE/EtOAc 10/1). ${ }^{1} \mathbf{H}$ NMR $\left(400 \mathrm{MHz} \mathrm{CDCl}_{3}\right) \delta 7.95-7.86(\mathrm{~m}, 2 \mathrm{H}), 7.68-7.62(\mathrm{~m}, 1 \mathrm{H}), 7.57-$ $7.46(\mathrm{~m}, 3 \mathrm{H}), 7.31(\mathrm{~d}, \mathrm{~J}=7.1 \mathrm{~Hz}, 1 \mathrm{H}), 2.70(\mathrm{dq}, \mathrm{J}=17.8,7.3 \mathrm{~Hz}, 2 \mathrm{H}), 2.51(\mathrm{dq}, \mathrm{J}=17.8,7.2 \mathrm{~Hz}, 2 \mathrm{H})$, $1.09(\mathrm{t}, \mathrm{J}=7.3 \mathrm{~Hz}, 6 \mathrm{H}) .{ }^{13} \mathbf{C ~ N M R}\left(100 \mathrm{MHz} \mathrm{CDCl}_{3}\right) \delta 176.9,135.5,134.6,130.7,129.4,128.8$, 127.7, 127.1, 126.7, 125.7, 121.5, 31.8, 9.0. HRMS calculated for $\mathrm{C}_{16} \mathrm{H}_{17} \mathrm{NO}_{2}[\mathrm{M}+\mathrm{H}]^{+}:$256.1332, found 256.1326 .

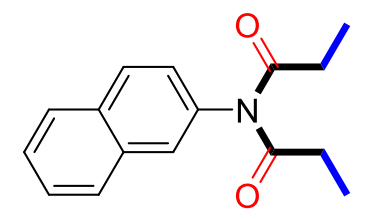

$\boldsymbol{N}$-(naphthalen-2-yl)- $\boldsymbol{N}$-propionylpropionamide (35): White solid, $96 \mathrm{mg}, 94 \%$ yield, $\mathrm{R}_{\mathrm{f}}=0.2$ (PE/EtOAc 10/1). ${ }^{1} \mathbf{H}$ NMR (400 MHz, $\left.\mathrm{CDCl}_{3}\right) \delta 7.91(\mathrm{~d}, \mathrm{~J}=8.6 \mathrm{~Hz}, 1 \mathrm{H}), 7.87(\mathrm{~d}, \mathrm{~J}=8.3 \mathrm{~Hz}, 1 \mathrm{H})$, $7.81(\mathrm{~d}, \mathrm{~J}=8.3 \mathrm{~Hz}, 1 \mathrm{H}), 7.62(\mathrm{~s}, 1 \mathrm{H}), 7.56-7.47(\mathrm{~m}, 2 \mathrm{H}), 7.21(\mathrm{~d}, \mathrm{~J}=8.6 \mathrm{~Hz}, 1 \mathrm{H}), 2.62(\mathrm{q}, \mathrm{J}=7.3$ $\mathrm{Hz}, 4 \mathrm{H}), 1.11(\mathrm{t}, \mathrm{J}=7.3 \mathrm{~Hz}, 6 \mathrm{H}) .{ }^{13} \mathbf{C ~ N M R}\left(100 \mathrm{MHz}, \mathrm{CDCl}_{3}\right) \delta 176.8,136.5,133.6,133.0,129.8$, 128.0, 127.9, 127.7, 127.0, 126.8, 126.6, 32.3, 9.0. HRMS calculated for $\mathrm{C}_{16} \mathrm{H}_{17} \mathrm{NO}_{2}[\mathrm{M}+\mathrm{H}]^{+}$: 256.1332 , found 256.1328 . 
<smiles>CCC(=O)N(C(=O)CC)c1ccc2c(c1)Cc1ccccc1-2</smiles>

$N$-(9H-fluoren-2-yl)- $N$-propionylpropionamide (36): White solid, $115 \mathrm{mg}, 98 \%$ yield, $\mathrm{R}_{\mathrm{f}}=0.2$ (PE/EtOAc 10/1). ${ }^{1} \mathbf{H}$ NMR (400 MHz, $\left.\mathrm{CDCl}_{3}\right) \delta 7.80(\mathrm{~d}, \mathrm{~J}=8.0 \mathrm{~Hz}, 1 \mathrm{H}), 7.76(\mathrm{~d}, \mathrm{~J}=7.5 \mathrm{~Hz}, 1 \mathrm{H})$, $7.51(\mathrm{~d}, \mathrm{~J}=7.4 \mathrm{~Hz}, 1 \mathrm{H}), 7.36(\mathrm{t}, \mathrm{J}=7.5 \mathrm{~Hz}, 1 \mathrm{H}), 7.31(\mathrm{~d}, \mathrm{~J}=7.3 \mathrm{~Hz}, 1 \mathrm{H}), 7.28(\mathrm{~s}, 1 \mathrm{H}), 7.11(\mathrm{~d}, \mathrm{~J}=$ $7.7 \mathrm{~Hz}, 1 \mathrm{H}), 3.88(\mathrm{~s}, 2 \mathrm{H}), 2.62(\mathrm{q}, \mathrm{J}=7.2 \mathrm{~Hz}, 4 \mathrm{H}), 1.12(\mathrm{t}, \mathrm{J}=7.3 \mathrm{~Hz}, 6 \mathrm{H}) .{ }^{13} \mathrm{C}$ NMR $(100 \mathrm{MHz}$, $\left.\mathrm{CDCl}_{3}\right) \delta 176.9,144.7,143.5,142.3,140.6,137.5,127.6,127.3,127.0,125.6,125.1,120.8,120.2$, 36.9, 32.2, 9.1. HRMS calculated for $\mathrm{C}_{19} \mathrm{H}_{19} \mathrm{NO}_{2}[\mathrm{M}+\mathrm{H}]^{+}: 294.1489$, found 294.1493 .<smiles>CCC(=O)N(C(=O)CC)c1ccc(C)nc1</smiles>

$\mathrm{N}$-(6-methylpy ridin-3-yl)- $\mathrm{N}$-propionylpropionamide (37): Colorless oil, $68 \mathrm{mg}, 77 \%$ yield, $\mathrm{R}_{\mathrm{f}}$ $=0.2(\mathrm{PE} / \mathrm{EtOAc} 5 / 1) .{ }^{1} \mathbf{H}$ NMR $\left(400 \mathrm{MHz}, \mathrm{CDCl}_{3}\right) \delta 8.28(\mathrm{~s}, 1 \mathrm{H}), 7.37(\mathrm{~d}, \mathrm{~J}=8.1 \mathrm{~Hz}, 1 \mathrm{H}), 7.27$ $(\mathrm{d}, \mathrm{J}=8.2 \mathrm{~Hz}, 1 \mathrm{H}), 2.63-2.55(\mathrm{~m}, 7 \mathrm{H}), 1.11(\mathrm{t}, \mathrm{J}=7.3 \mathrm{~Hz}, 6 \mathrm{H}) .{ }^{13} \mathbf{C ~ N M R}\left(100 \mathrm{MHz}, \mathrm{CDCl}_{3}\right) \delta$ $176.4,159.0,148.9,136.7,133.1,123.9,32.3,24.2,8.8$. HRMS calculated for $\mathrm{C}_{12} \mathrm{H}_{16} \mathrm{~N}_{2} \mathrm{O}_{2}[\mathrm{M}+\mathrm{H}]^{+}$: 221.1285 , found 221.1286 .<smiles>CCC(=O)N(C(=O)CC)c1ccc(C(=O)OC)s1</smiles>

Methyl 5-( $N$-propionylpropionamido)thiophene-2-carboxylate (38): Yellow oil, $76 \mathrm{mg}, 71 \%$ yield, $\mathrm{R}_{\mathrm{f}}=0.2(\mathrm{PE} / \mathrm{EtOAc} 10 / 1) .{ }^{1} \mathbf{H}$ NMR $\left(400 \mathrm{MHz}, \mathrm{CDCl}_{3}\right) \delta 7.71(\mathrm{~d}, \mathrm{~J}=3.9 \mathrm{~Hz}, 1 \mathrm{H}), 6.87(\mathrm{~d}$, $\mathrm{J}=3.9 \mathrm{~Hz}, 1 \mathrm{H}), 3.90(\mathrm{~s}, 3 \mathrm{H}), 2.70(\mathrm{q}, \mathrm{J}=7.3 \mathrm{~Hz}, 4 \mathrm{H}), 1.13(\mathrm{t}, \mathrm{J}=7.3 \mathrm{~Hz}, 6 \mathrm{H}) .{ }^{13} \mathbf{C}$ NMR $(100 \mathrm{MHz}$, $\left.\mathrm{CDCl}_{3}\right) \delta 176.1,161.9,145.8,133.7,132.3,128.4,52.41(\mathrm{~d}, \mathrm{~J}=2.0 \mathrm{~Hz}), 31.8,8.9$. HRMS calculated for $\mathrm{C}_{12} \mathrm{H}_{15} \mathrm{NO}_{4} \mathrm{~S}[\mathrm{M}+\mathrm{H}]^{+}: 270.0795$, found 270.0790 .<smiles>CCC(=O)N(C(=O)CC)c1sccc1C#N</smiles>

$\mathrm{N}$-(3-cyanothiophen-2-yl)- $\mathrm{N}$-propionylpropionamide (39): Colorless oil, $80 \mathrm{mg}, 85 \%$ yield, $\mathrm{R}_{\mathrm{f}}$ $=0.2(\mathrm{PE} / \mathrm{EtOAc} 10 / 1) .{ }^{1} \mathbf{H}$ NMR $\left(400 \mathrm{MHz}, \mathrm{CDCl}_{3}\right) \delta 7.46(\mathrm{~d}, \mathrm{~J}=5.8 \mathrm{~Hz}, 1 \mathrm{H}), 7.23(\mathrm{~d}, \mathrm{~J}=5.8 \mathrm{~Hz}$, 1H), $2.70(\mathrm{q}, \mathrm{J}=7.2 \mathrm{~Hz}, 4 \mathrm{H}), 1.17(\mathrm{t}, \mathrm{J}=7.2 \mathrm{~Hz}, 6 \mathrm{H}) .{ }^{13} \mathbf{C} \mathbf{N M R}\left(100 \mathrm{MHz}, \mathrm{CDCl}_{3}\right) \delta$ 175.5, 149.5, 127.4, 112.6, 111.4, 31.7, 8.8. HRMS calculated for $\mathrm{C}_{11} \mathrm{H}_{12} \mathrm{~N}_{2} \mathrm{O}_{2} \mathrm{~S}\left[\mathrm{M}+\mathrm{NH}_{4}\right]^{+}: 254.0958$, found 254.0949 .<smiles>CCC(=O)N(C(=O)CC)c1ccc2c(c1)OCO2</smiles>

$N$-(benzo $[d][1,3]$ dioxol-5-yl)- $N$-propionylpropionamide (40): Colorless oil, $85 \mathrm{mg}, 90 \%$ yield, $\mathrm{R}_{\mathrm{f}}=0.2(\mathrm{PE} / \mathrm{EtOAc} 5: 1) .{ }^{1} \mathbf{H}$ NMR $\left(400 \mathrm{MHz}, \mathrm{CDCl}_{3}\right) \delta 6.84(\mathrm{~d}, \mathrm{~J}=8.0 \mathrm{~Hz}, 1 \mathrm{H}), 6.65-6.55(\mathrm{~m}$, 
2H), $6.02(\mathrm{~s}, 2 \mathrm{H}), 2.61(\mathrm{q}, \mathrm{J}=7.2 \mathrm{~Hz}, 4 \mathrm{H}), 1.11(\mathrm{t}, \mathrm{J}=7.3 \mathrm{~Hz}, 6 \mathrm{H}) .{ }^{13} \mathbf{C ~ N M R}\left(100 \mathrm{MHz}, \mathrm{CDCl}_{3}\right) \delta$ 176.8, 148.6, 147.9, 132.6, 122.3, 109.7, 108.6, 101.9, 32.0, 9.0. HRMS calculated for $\mathrm{C}_{13} \mathrm{H}_{15} \mathrm{NO}_{4}$ $[\mathrm{M}+\mathrm{H}]^{+}$250.1074, found 250.1065 .<smiles>CCC(=O)N(C(=O)CC)c1ccc2ncccc2c1</smiles>

$N$-propionyl- $N$-(quinolin-6-yl)propionamide (41): White solid, $91 \mathrm{mg}, 89 \%$ yield, $\mathrm{R}_{\mathrm{f}}=0.2$ (PE/EtOAc 5/1). ${ }^{1} \mathbf{H}$ NMR (400 MHz, $\left.\mathrm{CDCl}_{3}\right) \delta 9.02-8.92(\mathrm{~m}, 1 \mathrm{H}), 8.20(\mathrm{~d}, \mathrm{~J}=8.9 \mathrm{~Hz}, 1 \mathrm{H}), 8.13$ $(\mathrm{d}, \mathrm{J}=8.3 \mathrm{~Hz}, 1 \mathrm{H}), 7.64(\mathrm{~s}, 1 \mathrm{H}), 7.51-7.40(\mathrm{~m}, 2 \mathrm{H}), 2.64(\mathrm{q}, \mathrm{J}=7.3 \mathrm{~Hz}, 4 \mathrm{H}), 1.13$ (t, J = 7.3 Hz, 6H). ${ }^{13} \mathrm{C}$ NMR $\left(100 \mathrm{MHz}, \mathrm{CDCl}_{3}\right) \delta 176.6,151.4,147.6,137.1,136.1,131.4,130.3,128.5,127.7$, 121.8, 32.3, 8.9. HRMS calculated for $\mathrm{C}_{15} \mathrm{H}_{16} \mathrm{~N}_{2} \mathrm{O}_{2}[\mathrm{M}+\mathrm{H}]^{+}: 227.1285$, found 257.1288.<smiles>CCC(=O)N(C(=O)CC)c1cnc2ccccc2c1</smiles>

$N$-propionyl- $N$-(quinolin-3-yl)propionamide (42): Colorless oil, $95 \mathrm{mg}, 93 \%$ yield, $\mathrm{R}_{\mathrm{f}}=0.2$ (PE/EtOAc 5/1). ${ }^{1} \mathbf{H}$ NMR $\left(400 \mathrm{MHz}, \mathrm{CDCl}_{3}\right) \delta 8.68(\mathrm{~d}, \mathrm{~J}=2.4 \mathrm{~Hz}, 1 \mathrm{H}), 8.17(\mathrm{~d}, \mathrm{~J}=8.5 \mathrm{~Hz}, 1 \mathrm{H})$, $7.98(\mathrm{~d}, \mathrm{~J}=2.4 \mathrm{~Hz}, 1 \mathrm{H}), 7.85-7.74(\mathrm{~m}, 2 \mathrm{H}), 7.59(\mathrm{t}, \mathrm{J}=7.5 \mathrm{~Hz}, 1 \mathrm{H}), 2.63(\mathrm{q}, \mathrm{J}=7.3 \mathrm{~Hz}, 4 \mathrm{H}), 1.13(\mathrm{t}$, $\mathrm{J}=7.3 \mathrm{~Hz}, 6 \mathrm{H}) .{ }^{13} \mathbf{C} \mathbf{N M R}\left(100 \mathrm{MHz}, \mathrm{CDCl}_{3}\right) \delta 176.4,150.5,147.5,135.5,132.5,130.5,129.4$, $127.9,127.9,127.6,32.5,8.9$. HRMS calculated for $\mathrm{C}_{15} \mathrm{H}_{16} \mathrm{~N}_{2} \mathrm{O}_{2}[\mathrm{M}+\mathrm{H}]^{+}: 257.1285$, found 257.1280 .<smiles>CCC(=O)Nc1cccc2cccnc12</smiles>

$\mathrm{N}$-(quinolin-8-yl)propionamide (43) ${ }^{2}$ : Colorless oil, $59 \mathrm{mg}, 74 \%$ yield, $\mathrm{R}_{\mathrm{f}}=0.2(\mathrm{PE} / \mathrm{EtOAc}$ 2/1). ${ }^{1} \mathbf{H}$ NMR $\left(400 \mathrm{MHz}, \mathrm{CDCl}_{3}\right) \delta 9.81(\mathrm{~s}, 1 \mathrm{H}), 8.85-8.72(\mathrm{~m}, 2 \mathrm{H}), 8.13(\mathrm{~d}, \mathrm{~J}=8.2 \mathrm{~Hz}, 1 \mathrm{H}), 7.55$ -7.39 (m, 3H), 2.59 (q, J = 7.5 Hz, 2H), 1.34 (t, J = 7.5 Hz, 3H). ${ }^{13} \mathbf{C} \mathbf{N M R}\left(100 \mathrm{MHz}, \mathrm{CDCl}_{3}\right) \delta$ 172.5, 148.1,138.3, 136.4, 134.6, 127.9,127.4, 121.6,121.3,116.4,31.6,9.8.<smiles>CCC(=O)N(C(=O)CC)c1nccs1</smiles>

$N$-propionyl- $N$-(thiazol-2-yl)propionamide (44): Colorless oil, $56 \mathrm{mg}, 66 \%$ yield, $\mathrm{R}_{\mathrm{f}}=0.2$ (PE/EtOAc 5/1). ${ }^{1} \mathbf{H}$ NMR (400 MHz, $\left.\mathrm{CDCl}_{3}\right) \delta 7.73(\mathrm{~d}, \mathrm{~J}=3.5 \mathrm{~Hz}, 1 \mathrm{H}), 7.47(\mathrm{~d}, \mathrm{~J}=3.4 \mathrm{~Hz}, 1 \mathrm{H})$, 2.67 (q, J = 7.2 Hz, 4H), 1.15 (t, J = 7.3 Hz, 6H). $\left.{ }^{13} \mathbf{C ~ N M R ~ ( 1 0 0 ~ M H z , ~ C D C l ~}\right) \delta 175.8,159.3$, 140.5, 122.1,31.6, 8.7. HRMS calculated for $\mathrm{C}_{9} \mathrm{H}_{12} \mathrm{~N}_{2} \mathrm{O}_{2} \mathrm{~S}[\mathrm{M}+\mathrm{H}]^{+}: 213.0692$, found 213.0683 .<smiles>CCC(=O)Nc1nccs1</smiles>

$N$-(thiazol-2-yl)propionamide $(\mathbf{4 5})^{3}$ : White solid, $20 \mathrm{mg}, 32 \%$ yield, $\mathrm{R}_{\mathrm{f}}=0.2(\mathrm{PE} / \mathrm{EtOAc} 2 / 1)$. 
${ }^{1} \mathbf{H}$ NMR $\left(400 \mathrm{MHz}, \mathrm{CDCl}_{3}\right) \delta 12.68(\mathrm{~s}, 1 \mathrm{H}), 7.44(\mathrm{~d}, \mathrm{~J}=3.7 \mathrm{~Hz}, 1 \mathrm{H}), 7.00(\mathrm{~d}, \mathrm{~J}=3.5 \mathrm{~Hz}, 1 \mathrm{H}), 2.61$ $(\mathrm{q}, \mathrm{J}=7.5 \mathrm{~Hz}, 2 \mathrm{H}), 1.31$ (t, $\mathrm{J}=7.5 \mathrm{~Hz}, 3 \mathrm{H}) .{ }^{13} \mathbf{C} \mathbf{~ N M R}\left(100 \mathrm{MHz}, \mathrm{CDCl}_{3}\right) \delta 172.0,160.4,136.1$, 113.4, 29.3,9.2.<smiles>CCC(=O)N(C(=O)CC)c1nc2ccccc2s1</smiles>

$N$-(benzo $[d]$ thiazol-2-yl)- $N$-propionylpropionamide (46): Colorless oil, $67 \mathrm{mg}, 64 \%$ yield, $\mathrm{R}_{\mathrm{f}}$ $=0.2\left(\mathrm{PE} /\right.$ EtOAc 5/1). ${ }^{1} \mathbf{H}$ NMR $\left(400 \mathrm{MHz}, \mathrm{CDCl}_{3}\right) \delta 8.00(\mathrm{~d}, \mathrm{~J}=8.0 \mathrm{~Hz}, 1 \mathrm{H}), 7.87(\mathrm{~d}, \mathrm{~J}=7.9 \mathrm{~Hz}$, $1 \mathrm{H}$ ), 7.51 (t, J = 7.7 Hz, 1H), 7.44 (t, J = 7.7 Hz, 1H), 2.75 (q, J = 7.2 Hz, 4H), 1.18 (t, J = 7.2 Hz, 6H). ${ }^{13}$ C NMR $\left(100 \mathrm{MHz}, \mathrm{CDCl}_{3}\right) \delta 175.5,159.1,149.6,135.9,126.5,126.0,123.6,121.7,31.7,8.7$. HRMS calculated for $\mathrm{C}_{13} \mathrm{H}_{14} \mathrm{~N}_{2} \mathrm{O}_{2} \mathrm{~S}[\mathrm{M}+\mathrm{H}]^{+}: 263.0849$, found 263.0845 .<smiles>CCC(=O)Nc1nc2ccccc2s1</smiles>

$N$-(benzo $[d]$ thiazol-2-yl)propionamide $(47)^{4}$ : White solid, $26 \mathrm{mg}, 32 \%$ yield, $\mathrm{R}_{\mathrm{f}}=0.2$ (PE/EtOAc 2/1). ${ }^{1} \mathbf{H}$ NMR $\left(400 \mathrm{MHz}, \mathrm{CDCl}_{3}\right) \delta 11.72(\mathrm{~s}, 1 \mathrm{H}), 7.86(\mathrm{~d}, \mathrm{~J}=7.9 \mathrm{~Hz}, 1 \mathrm{H}), 7.75(\mathrm{~d}, \mathrm{~J}=$ $8.1 \mathrm{~Hz}, 1 \mathrm{H}), 7.46(\mathrm{t}, \mathrm{J}=7.7 \mathrm{~Hz}, 1 \mathrm{H}), 7.34(\mathrm{t}, \mathrm{J}=7.6 \mathrm{~Hz}, 1 \mathrm{H}), 2.51(\mathrm{q}, \mathrm{J}=7.5 \mathrm{~Hz}, 2 \mathrm{H}), 1.24(\mathrm{t}, \mathrm{J}=7.5$ $\mathrm{Hz}, 3 \mathrm{H}) .{ }^{13} \mathbf{C} \mathbf{N M R}\left(100 \mathrm{MHz}, \mathrm{CDCl}_{3}\right) \delta$ 172.7, 160.0, 147.7, 131.9, 126.4, 124.0, 121.7, 120.2, 29.7 , 9.0 .

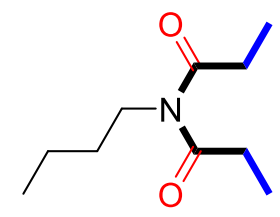

$N$-butyl- $N$-propionylpropionamide (48): Colorless oil, $42 \mathrm{mg}, 57 \%$ yield, $\mathrm{R}_{\mathrm{f}}=0.3$ (PE/EtOAc 20/1). ${ }^{1} \mathbf{H}$ NMR (400 MHz, $\left.\mathrm{CDCl}_{3}\right) \delta 3.65(\mathrm{t}, \mathrm{J}=7.6 \mathrm{~Hz}, 2 \mathrm{H}), 2.72(\mathrm{q}, \mathrm{J}=7.2 \mathrm{~Hz}, 4 \mathrm{H}), 1.53$ (p, J = 8.0, 7.6 Hz, 2H), 1.35 (p, J = 8.0, 7.2 Hz, 2H), 1.16 (t, J = 7.2 Hz, 6H), 0.95 (t, J = 7.2 Hz, 3H). ${ }^{13} \mathbf{C}$ NMR $\left(100 \mathrm{MHz}, \mathrm{CDCl}_{3}\right) \delta 176.9,44.0,31.4,31.3,20.2,13.7,9.2$. HRMS calculated for $\mathrm{C}_{10} \mathrm{H}_{19} \mathrm{NO}_{2}[\mathrm{M}+\mathrm{H}]^{+}: 186.1489$, found 186.1484 .

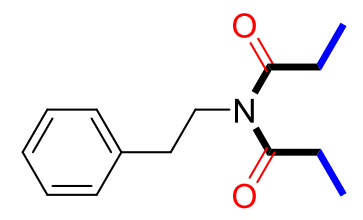

$N$-phenethyl- $N$-propionylpropionamide $(49)^{5}$ : Colorless oil, $43 \mathrm{mg}, 46 \%$ yield, $\mathrm{R}_{\mathrm{f}}=0.2$ (PE/EtOAc 20/1). ${ }^{1} \mathbf{H}$ NMR (400 MHz, $\left.\mathrm{CDCl}_{3}\right) \delta 7.35-7.28(\mathrm{~m}, 2 \mathrm{H}), 7.27-7.17$ (m, 3H), 3.87 (t, $\mathrm{J}=7.8 \mathrm{~Hz}, 2 \mathrm{H}), 2.85(\mathrm{t}, \mathrm{J}=7.8 \mathrm{~Hz}, 2 \mathrm{H}), 2.66(\mathrm{q}, \mathrm{J}=7.2 \mathrm{~Hz}, 4 \mathrm{H}), 1.13(\mathrm{t}, \mathrm{J}=7.2 \mathrm{~Hz}, 6 \mathrm{H}) .{ }^{13} \mathbf{C} \mathbf{N M R}$ $\left(100 \mathrm{MHz}, \mathrm{CDCl}_{3}\right) \delta 176.9,138.3,128.9,128.7,126.7,46.0,35.5,31.4,9.2$.

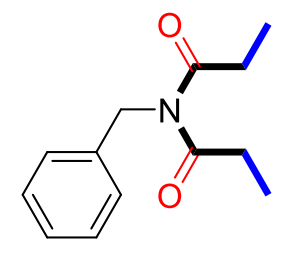

$N$-benzyl- $N$-propionylpropionamide (50): Colorless oil, $49 \mathrm{mg}, 56 \%$ yield, $\mathrm{R}_{\mathrm{f}}=0.2(\mathrm{PE} / \mathrm{EtOAc}$ 
20/1). ${ }^{1} \mathbf{H}$ NMR $\left(400 \mathrm{MHz}, \mathrm{CDCl}_{3}\right) \delta 7.32(\mathrm{t}, \mathrm{J}=7.3 \mathrm{~Hz}, 2 \mathrm{H}), 7.28-7.22(\mathrm{~m}, 1 \mathrm{H}), 7.12(\mathrm{~d}, \mathrm{~J}=7.4$ $\mathrm{Hz}, 2 \mathrm{H}), 4.98$ (s, 2H), 2.74 (q, J = 7.0 Hz, 4H), 1.13 (t, J = 7.1 Hz, 6H). $\left.{ }^{13} \mathbf{C ~ N M R ~ ( 1 0 0 ~ M H z , ~} \mathrm{CDCl}_{3}\right)$ $\delta$ 177.2, 137.3, 128.8, 127.3, 126.0, 46.9, 31.6, 9.1. HRMS calculated for $\mathrm{C}_{13} \mathrm{H}_{17} \mathrm{NO}_{2}[\mathrm{M}+\mathrm{H}]^{+}$: 220.1332 , found 220.1325 .<smiles>CCC(=O)N(Cc1ccco1)C(=O)CC</smiles>

$N$-(furan-2-ylmethyl)- $N$-propionylpropionamide (51): Colorless oil, $49 \mathrm{mg}, 59 \%$ yield, $\mathrm{R}_{\mathrm{f}}=$ 0.2 (PE/EtOAc 10/1). ${ }^{1} \mathbf{H}$ NMR $\left(400 \mathrm{MHz}, \mathrm{CDCl}_{3}\right) \delta 7.32(\mathrm{~d}, \mathrm{~J}=1.8 \mathrm{~Hz}, 1 \mathrm{H}), 6.31(\mathrm{dd}, \mathrm{J}=3.3,1.8$ $\mathrm{Hz}, 1 \mathrm{H}), 6.24(\mathrm{~d}, \mathrm{~J}=3.2 \mathrm{~Hz}, 1 \mathrm{H}), 4.90(\mathrm{~s}, 2 \mathrm{H}), 2.83(\mathrm{q}, \mathrm{J}=7.2 \mathrm{~Hz}, 4 \mathrm{H}), 1.16(\mathrm{t}, \mathrm{J}=7.2 \mathrm{~Hz}, 6 \mathrm{H}) .{ }^{13} \mathrm{C}$ NMR $\left(100 \mathrm{MHz}, \mathrm{CDCl}_{3}\right) \delta 176.7,150.7,142.0,110.5,108.2,40.4,31.4,9.1$. HRMS calculated for $\mathrm{C}_{11} \mathrm{H}_{15} \mathrm{NO}_{3}[\mathrm{M}+\mathrm{Na}]^{+}: 232.0944$, found 232.0953.<smiles>CCC(=O)N(C(=O)CC)C1CCCC1</smiles>

$N$-cyclopentyl- $N$-propionylpropionamide (52): Colorless oil, $33 \mathrm{mg}, 42 \%$ yield, $\mathrm{R}_{\mathrm{f}}=0.2$ (PE/EtOAc 20/1). ${ }^{1} \mathbf{H}$ NMR (400 MHz, $\left.\mathrm{CDCl}_{3}\right) \delta 4.25(\mathrm{p}, \mathrm{J}=8.3 \mathrm{~Hz}, 1 \mathrm{H}), 2.65$ (q, J = 7.3 Hz, 4H), $2.04-1.87(\mathrm{~m}, 4 \mathrm{H}), 1.86-1.73(\mathrm{~m}, 2 \mathrm{H}), 1.61-1.47(\mathrm{~m}, 2 \mathrm{H}), 1.16(\mathrm{t}, \mathrm{J}=7.3 \mathrm{~Hz}, 6 \mathrm{H}) .{ }^{13} \mathbf{C}$ NMR $\left(100 \mathrm{MHz}, \mathrm{CDCl}_{3}\right) \delta 178.0,57.9,31.9,29.5,25.6,9.5$. HRMS calculated for $\mathrm{C}_{11} \mathrm{H}_{19} \mathrm{NO}_{2}[\mathrm{M}+\mathrm{Na}]^{+}$: 220.1308 , found 220.1307 .<smiles>CCC(=O)N(C(=O)CC)c1ccc(C(=O)OC2CC(C)CCC2C(C)C)cc1</smiles>

(2S,5R)-2-isopropyl-5-methylcyclohexyl $\quad 4$-( $N$-propionylpropionamido)benzoate $\quad(56)$ : Colorless oil, $133 \mathrm{mg}, 86 \%$ yield, $\mathrm{R}_{\mathrm{f}}=0.2(\mathrm{PE} / \mathrm{EtOAc} 20 / 1) .{ }^{\mathbf{1}} \mathbf{H} \mathbf{N M R}\left(400 \mathrm{MHz}, \mathrm{CDCl}_{3}\right) \delta 8.14$ $(\mathrm{d}, \mathrm{J}=8.2 \mathrm{~Hz}, 2 \mathrm{H}), 7.22(\mathrm{~d}, \mathrm{~J}=8.1 \mathrm{~Hz}, 2 \mathrm{H}), 4.96(\mathrm{td}, \mathrm{J}=10.9,4.3 \mathrm{~Hz}, 1 \mathrm{H}), 2.58(\mathrm{q}, \mathrm{J}=7.3 \mathrm{~Hz}, 4 \mathrm{H})$, $2.17-2.08(\mathrm{~m}, 1 \mathrm{H}), 1.96(\mathrm{pd}, \mathrm{J}=7.1,2.4 \mathrm{~Hz}, 1 \mathrm{H}), 1.81-1.68(\mathrm{~m}, 2 \mathrm{H}), 1.65-1.49(\mathrm{~m}, 2 \mathrm{H}), 1.18-$ $1.08(\mathrm{~m}, 8 \mathrm{H}), 0.99-0.88(\mathrm{~m}, 7 \mathrm{H}), 0.80(\mathrm{~d}, \mathrm{~J}=6.9 \mathrm{~Hz}, 3 \mathrm{H}) .{ }^{13} \mathbf{C} \mathbf{N M R}\left(100 \mathrm{MHz}, \mathrm{CDCl}_{3}\right) \delta 176.2$, 165.1, 143.1, 131.2, $131.0,129.1,75.3,47.3,40.9,34.3,32.2,31.5,26.5,23.6,22.0,20.8,16.5,8.9$. HRMS calculated for $\mathrm{C}_{23} \mathrm{H}_{33} \mathrm{NO}_{4}[\mathrm{M}+\mathrm{H}]^{+}: 388.2482$, found 388.2484 .<smiles>CCC(=O)N(C(=O)CC)c1ccc(C(=O)OCC23OCC4OC(C)(C)OC4[C@H](OC2(C)C)O3)cc1</smiles>

((3aR,5aS,8aS,8bR)-2,2,7,7-tetramethyltetrahydro-3aH-bis([1,3]dioxolo)[4,5-b:4',5'-d]pyra n-3a-yl)methyl 4-( $N$-propionylpropionamido)benzoate (57): Colorless oil, $184 \mathrm{mg}, 94 \%$ yield, 
$\mathrm{R}_{\mathrm{f}}=0.2(\mathrm{PE} / \mathrm{EtOAc} 10 / 1) .{ }^{1} \mathbf{H}$ NMR $\left(400 \mathrm{MHz}, \mathrm{CDCl}_{3}\right) \delta 8.18(\mathrm{~d}, \mathrm{~J}=8.4 \mathrm{~Hz}, 2 \mathrm{H}), 7.23(\mathrm{~d}, \mathrm{~J}=8.5$ $\mathrm{Hz}, 2 \mathrm{H}), 4.71-4.62(\mathrm{~m}, 2 \mathrm{H}), 4.44(\mathrm{~d}, \mathrm{~J}=2.6 \mathrm{~Hz}, 1 \mathrm{H}), 4.37(\mathrm{~d}, \mathrm{~J}=11.8 \mathrm{~Hz}, 1 \mathrm{H}), 4.30-4.25(\mathrm{~m}, 1 \mathrm{H})$, $3.96(\mathrm{dd}, \mathrm{J}=13.0,1.9 \mathrm{~Hz}, 1 \mathrm{H}), 3.81(\mathrm{dd}, \mathrm{J}=13.0,0.8 \mathrm{~Hz}, 1 \mathrm{H}), 2.57(\mathrm{q}, \mathrm{J}=7.3 \mathrm{~Hz}, 4 \mathrm{H}), 1.56(\mathrm{~s}, 3 \mathrm{H})$, $1.46(\mathrm{~s}, 3 \mathrm{H}), 1.40(\mathrm{~s}, 3 \mathrm{H}), 1.35$ (s, 3H), $1.11(\mathrm{t}, \mathrm{J}=7.3 \mathrm{~Hz}, 6 \mathrm{H}) .{ }^{13} \mathbf{C ~ N M R}\left(100 \mathrm{MHz}, \mathrm{CDCl}_{3}\right) \delta$ 176.2, 165.0, 143.5, 131.2, 130.3, 129.2, 109.2, 108.9, 101.5, 70.7, 70.6, 70.1, 65.9, 61.4, 32.2, 26.5, 25.9, 25.5, 24.0, 8.9. HRMS calcula ted for $\mathrm{C}_{25} \mathrm{H}_{33} \mathrm{NO}_{9}[\mathrm{M}+\mathrm{H}]^{+} 492.2228$, found 492.2212 .

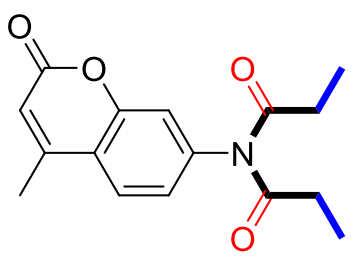

$N$-(4-methyl-2-oxo-2H-chromen-7-yl)- $N$-propionylpropionamide (58): White solid, $46 \mathrm{mg}$, $40 \%$ yield, $\mathrm{R}_{\mathrm{f}}=0.2(\mathrm{PE} / \mathrm{EtOAc} 10 / 1) .{ }^{1} \mathbf{H}$ NMR $\left(400 \mathrm{MHz} \mathrm{CDCl}_{3}\right) \delta 7.71(\mathrm{~d}, \mathrm{~J}=8.2 \mathrm{~Hz}, 1 \mathrm{H})$, $7.15(\mathrm{~s}, 1 \mathrm{H}), 7.11(\mathrm{~d}, \mathrm{~J}=8.4 \mathrm{~Hz}, 1 \mathrm{H}), 6.35(\mathrm{~s}, 1 \mathrm{H}), 2.61(\mathrm{q}, \mathrm{J}=7.2 \mathrm{~Hz}, 4 \mathrm{H}), 2.47(\mathrm{~s}, 3 \mathrm{H}), 1.12(\mathrm{t}, \mathrm{J}=$ $7.2 \mathrm{~Hz}, 6 \mathrm{H}) .{ }^{13} \mathrm{C}$ NMR $\left(100 \mathrm{MHz}, \mathrm{CDCl}_{3}\right) \delta 176.1,156.0,154.1,151.6,142.1,125.8,125.1,120.3$, $117.8,115.9,32.2,18.7,8.9$. HRMS calculated for $\mathrm{C}_{16} \mathrm{H}_{17} \mathrm{NO}_{4}[\mathrm{M}+\mathrm{H}]^{+}$: 288.1230 , found 288.1219 .

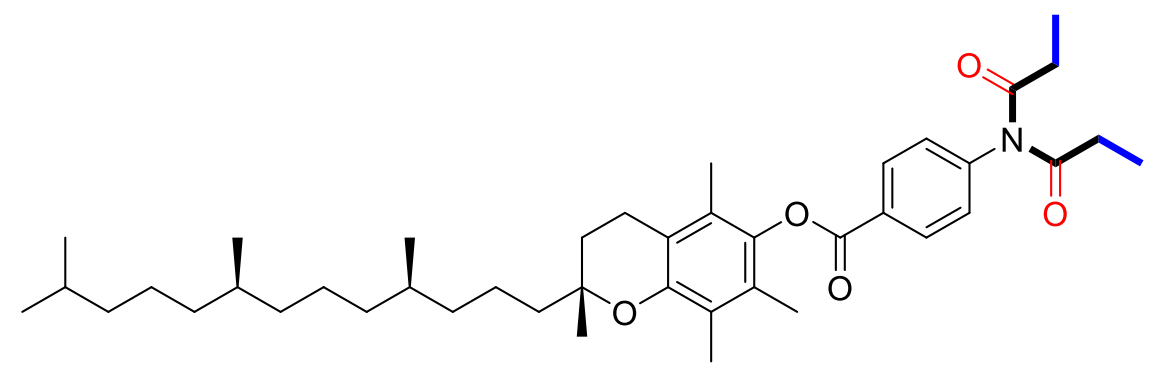

(R)-2,5,7,8-tetramethyl-2-((4R,8R)-4,8,12-trimethyltridecyl)chroman-6-yl

4-(N-propionylpropionamido)benzoate (59): Colorless oil, $198 \mathrm{mg}, 75 \%$ yield, $\mathrm{R}_{\mathrm{f}}=0.2$ (PE/EtOAc 10/1). ${ }^{1} \mathbf{H}$ NMR (400 MHz, $\left.\mathrm{CDCl}_{3}\right) \delta 8.34(\mathrm{~d}, \mathrm{~J}=8.0 \mathrm{~Hz}, 2 \mathrm{H}), 7.30(\mathrm{~d}, \mathrm{~J}=8.1 \mathrm{~Hz}, 2 \mathrm{H})$, $2.62(\mathrm{q}, \mathrm{J}=7.2 \mathrm{~Hz}, 6 \mathrm{H}), 2.13(\mathrm{~s}, 3 \mathrm{H}), 2.07(\mathrm{~s}, 3 \mathrm{H}), 2.02(\mathrm{~s}, 3 \mathrm{H}), 1.90-1.73(\mathrm{~m}, 2 \mathrm{H}), 1.66-1.35(\mathrm{~m}$, $7 \mathrm{H}), 1.35-1.20(\mathrm{~m}, 11 \mathrm{H}), 1.19-1.02(\mathrm{~m}, 12 \mathrm{H}), 0.94-0.80(\mathrm{~m}, 12 \mathrm{H}) .{ }^{13} \mathbf{C ~ N M R}\left(100 \mathrm{MHz}, \mathrm{CDCl}_{3}\right)$ $\delta$ 176.2, 164.2, 149.6, 143.8, 140.5, 131.6, 130.1, 129.4, 126.8, 125.1, 123.2, 117.6, 75.2, 39.4, 37.6, 37.6, 37.5, 37.4, 37.3, 32.8, 32.8, 32.7, 32.3, 28.0, 24.9, 24.8, 24.5, $22.8,22.7,21.1,20.7,19.8,19.7$, $19.7,19.7,19.7,13.1,12.3,11.9,8.9$. HRMS calculated for $\mathrm{C}_{42} \mathrm{H}_{63} \mathrm{NO}_{5}[\mathrm{M}+\mathrm{H}]^{+}: 662.4779$, found 662.4777 .<smiles>CCC(=O)N1C(=O)CCC(CC)(c2ccc(N(C(=O)CC)C(=O)CC)cc2)C1=O</smiles>

$\mathrm{N}$-(4-(3-ethyl-2,6-dioxopiperidin-3-yl)phenyl)- $\mathrm{N}$-propionylpropionamide (60): Yellow oil, $114 \mathrm{mg}, 71 \%$ yield, $\mathrm{R}_{\mathrm{f}}=0.2(\mathrm{PE} / \mathrm{EtOAc} 10 / 1) .{ }^{1} \mathbf{H}$ NMR $\left(400 \mathrm{MHz}, \mathrm{CDCl}_{3}\right) \delta 7.39(\mathrm{~d}, \mathrm{~J}=8.6 \mathrm{~Hz}$, $2 \mathrm{H}), 7.18(\mathrm{~d}, \mathrm{~J}=8.5 \mathrm{~Hz}, 2 \mathrm{H}), 2.81-2.65(\mathrm{~m}, 3 \mathrm{H}), 2.58(\mathrm{q}, \mathrm{J}=7.2 \mathrm{~Hz}, 4 \mathrm{H}), 2.54-2.26(\mathrm{~m}, 3 \mathrm{H}), 2.09$ $(\mathrm{dq}, \mathrm{J}=14.7,7.4 \mathrm{~Hz}, 1 \mathrm{H}), 1.95(\mathrm{dq}, \mathrm{J}=14.6,7.4 \mathrm{~Hz}, 1 \mathrm{H}), 1.22(\mathrm{t}, \mathrm{J}=7.2 \mathrm{~Hz}, 3 \mathrm{H}), 1.11(\mathrm{t}, \mathrm{J}=7.2 \mathrm{~Hz}$, $6 \mathrm{H}), 0.89(\mathrm{t}, \mathrm{J}=7.4 \mathrm{~Hz}, 3 \mathrm{H}) .{ }^{13} \mathbf{C}$ NMR $\left(100 \mathrm{MHz}, \mathrm{CDCl}_{3}\right) \delta 179.0,176.5,174.2,171.0,139.3$, 
138.6, 129.7, 127.5, 51.3, 34.5, 33.1, 32.2, 29.6, 26.5, 9.1, 8.9, 7.8. HRMS calculated for $\mathrm{C}_{22} \mathrm{H}_{28} \mathrm{~N}_{2} \mathrm{O}_{5}[\mathrm{M}+\mathrm{H}]^{+}: 401.2071$, found 401.2070 .

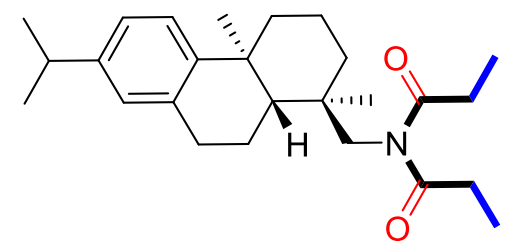

$N$-(((1R,4aS,10aR)-7-isopropyl-1,4a-dimethyl-1,2,3,4,4a,9,10,10a-octahydrophenanthren-1yl)methyl)- $N$-propionylpropionamide (61): Colorless oil, $82 \mathrm{mg}, 52 \%$ yield, $\mathrm{R}_{\mathrm{f}}=0.2$ (PE/EtOAc 20/1). ${ }^{1} \mathbf{H}$ NMR $\left(400 \mathrm{MHz}, \mathrm{CDCl}_{3}\right) \delta 7.14(\mathrm{~d}, \mathrm{~J}=8.1 \mathrm{~Hz}, 1 \mathrm{H}), 6.98(\mathrm{~d}, \mathrm{~J}=8.1 \mathrm{~Hz}, 1 \mathrm{H})$, $6.89(\mathrm{~s}, 1 \mathrm{H}), 3.76(\mathrm{~s}, 2 \mathrm{H}), 3.00-2.76(\mathrm{~m}, 3 \mathrm{H}), 2.66(\mathrm{q}, \mathrm{J}=7.5 \mathrm{~Hz}, 4 \mathrm{H}), 2.26(\mathrm{~d}, \mathrm{~J}=12.7 \mathrm{~Hz}, 1 \mathrm{H})$, $2.00(\mathrm{dd}, \mathrm{J}=13.5,6.9 \mathrm{~Hz}, 1 \mathrm{H}), 1.86-1.58(\mathrm{~m}, 3 \mathrm{H}), 1.39(\mathrm{~d}, \mathrm{~J}=12.5 \mathrm{~Hz}, 3 \mathrm{H}), 1.22(\mathrm{~d}, \mathrm{~J}=8.3 \mathrm{~Hz}$, $10 \mathrm{H}), 1.14(\mathrm{t}, \mathrm{J}=7.2 \mathrm{~Hz}, 6 \mathrm{H}), 0.93(\mathrm{~s}, 3 \mathrm{H}) .{ }^{13} \mathrm{C}$ NMR $\left(100 \mathrm{MHz}, \mathrm{CDCl}_{3}\right) \delta$ 178.3, 1472, 145.7, 134.6, 126.9, 123.9, 123.8, 53.6, 46.6, 39.8, 38.1, 37.9, 37.2, 33.5, 31.3, 30.1, 25.8, 24.0, 24.0, 19.6, 18.5, 18.4, 9.6. HRMS calculated for $\mathrm{C}_{26} \mathrm{H}_{39} \mathrm{NO}_{2}[\mathrm{M}+\mathrm{H}]^{+}: 398.3054$, found 398.3052 .<smiles>CCC(=O)Oc1ccccc1</smiles>

Phenyl propionate (62) $)^{6}$ : Colorless oil, $57 \mathrm{mg}, 95 \%$ yield, $\mathrm{R}_{\mathrm{f}}=0.2(\mathrm{PE} / \mathrm{EtOAc} 20 / 1) .{ }^{1} \mathbf{H}$ NMR $\left(400 \mathrm{MHz}, \mathrm{CDCl}_{3}\right) \delta 7.36(\mathrm{t}, \mathrm{J}=7.7 \mathrm{~Hz}, 2 \mathrm{H}), 7.21(\mathrm{t}, \mathrm{J}=7.5 \mathrm{~Hz}, 1 \mathrm{H}), 7.08(\mathrm{~d}, \mathrm{~J}=7.9 \mathrm{~Hz}, 2 \mathrm{H}), 2.58$ $(\mathrm{q}, \mathrm{J}=7.5 \mathrm{~Hz}, 2 \mathrm{H}), 1.26(\mathrm{t}, \mathrm{J}=7.5 \mathrm{~Hz}, 3 \mathrm{H}) .{ }^{13} \mathbf{C} \mathbf{N M R}\left(100 \mathrm{MHz}, \mathrm{CDCl}_{3}\right) \delta 173.0,150.8,129.4$, $125.7,121.6,27.8,9.1$.<smiles>CCC(=O)Oc1ccc(OC)cc1</smiles>

4-Methoxyphenyl propionate (63) ${ }^{7}$ : Colorless oil, $64 \mathrm{mg}$, 89\% yield, $\mathrm{R}_{\mathrm{f}}=0.2$ (PE/EtOAc 10/1). ${ }^{1} \mathbf{H}$ NMR $\left(400 \mathrm{MHz}, \mathrm{CDCl}_{3}\right) \delta 6.99(\mathrm{~d}, \mathrm{~J}=8.6 \mathrm{~Hz}, 2 \mathrm{H}), 6.87(\mathrm{~d}, \mathrm{~J}=8.6 \mathrm{~Hz}, 2 \mathrm{H}), 3.76(\mathrm{~s}, 3 \mathrm{H}), 2.55$ $(\mathrm{q}, \mathrm{J}=7.5 \mathrm{~Hz}, 2 \mathrm{H}), 1.24(\mathrm{t}, \mathrm{J}=7.5 \mathrm{~Hz}, 3 \mathrm{H}) .{ }^{13} \mathbf{C} \mathbf{N M R}\left(100 \mathrm{MHz}, \mathrm{CDCl}_{3}\right) \delta 173.3,157.2,144.3$, $122.3,114.4,55.6,27.7,9.1$.<smiles>CCC(=O)Oc1ccccc1I</smiles>

2-Iodophenyl propionate (64): Colorless oil, $90 \mathrm{mg}$, 82\% yield, $\mathrm{R}_{\mathrm{f}}=0.2(\mathrm{PE} / \mathrm{EtOAc} 20 / 1) .{ }^{1} \mathbf{H}$ NMR $\left(400 \mathrm{MHz}, \mathrm{CDCl}_{3}\right) \delta 7.81(\mathrm{~d}, \mathrm{~J}=7.9 \mathrm{~Hz}, 1 \mathrm{H}), 7.34(\mathrm{t}, \mathrm{J}=7.7 \mathrm{~Hz}, 1 \mathrm{H}), 7.09(\mathrm{~d}, \mathrm{~J}=8.0 \mathrm{~Hz}$, $1 \mathrm{H}), 6.96(\mathrm{t}, \mathrm{J}=7.6 \mathrm{~Hz}, 1 \mathrm{H}), 2.65(\mathrm{q}, \mathrm{J}=7.5 \mathrm{~Hz}, 2 \mathrm{H}), 1.31(\mathrm{t}, \mathrm{J}=7.6 \mathrm{~Hz}, 3 \mathrm{H}),{ }^{13} \mathbf{C} \mathbf{N M R}(100 \mathrm{MHz}$, $\left.\mathrm{CDCl}_{3}\right) \delta 172.0,151.2,139.4,129.5,127.6,123.1,90.6,28.0,9.1$. HRMS calculated for $\mathrm{C}_{9} \mathrm{H}_{9} \mathrm{IO}_{2}$ $\left[\mathrm{M}+\mathrm{NH}_{4}\right]^{+}: 293.9985$, found 293.9979 .

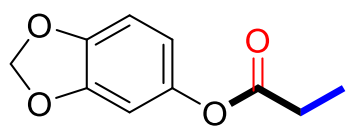

Benzo[d][1,3]dioxol-5-yl propionate $(\mathbf{6 5})^{8}$ : Colorless oil, $72 \mathrm{mg}, 93 \%$ yield, $\mathrm{R}_{\mathrm{f}}=0.2$ (PE/EtOAc 10/1). ${ }^{1}$ H NMR (400 MHz, CDCl $\left.{ }_{3}\right) \delta 6.75(\mathrm{~d}, \mathrm{~J}=8.4 \mathrm{~Hz}, 1 \mathrm{H}), 6.59(\mathrm{~s}, 1 \mathrm{H}), 6.51(\mathrm{~d}, \mathrm{~J}=$ $8.4 \mathrm{~Hz}, 1 \mathrm{H}), 5.95$ (s, 2H), 2.54 (q, J = $7.5 \mathrm{~Hz}, 2 \mathrm{H}), 1.32-1.19$ (m, 3H). ${ }^{13} \mathbf{C}$ NMR $(100 \mathrm{MHz}$, $\left.\mathrm{CDCl}_{3}\right) \delta 173.2,148.0,145.3,145.1,113.9,107.9,103.7,101.7,27.6,9.1$. 


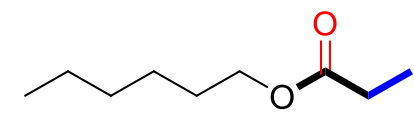

Hexyl propionate (66) ${ }^{9}$ : Colorless oil, $42 \mathrm{mg}, 76 \%$ yield, $\mathrm{R}_{\mathrm{f}}=0.2(\mathrm{PE} / \mathrm{EtOAc} 20 / 1) .{ }^{\mathbf{1}} \mathbf{H}$ NMR $\left(400 \mathrm{MHz}, \mathrm{CDCl}_{3}\right) \delta 4.07$ (t, J = 6.7 Hz, 2H), $2.32(\mathrm{q}, \mathrm{J}=7.6 \mathrm{~Hz}, 2 \mathrm{H}), 1.62(\mathrm{p}, \mathrm{J}=6.9 \mathrm{~Hz}, 2 \mathrm{H}), 1.42$ $-1.24(\mathrm{~m}, 6 \mathrm{H}), 1.14(\mathrm{t}, \mathrm{J}=7.6 \mathrm{~Hz}, 3 \mathrm{H}), 0.89(\mathrm{t}, \mathrm{J}=6.5 \mathrm{~Hz}, 3 \mathrm{H}) .{ }^{13} \mathbf{C} \mathbf{N M R}\left(100 \mathrm{MHz}, \mathrm{CDCl}_{3}\right) \delta$ 174.6, 64.5,31.4, 28.6, 27.6, 25.6, 22.5, 14.0,9.2.<smiles>CCC(=O)OCc1ccccc1</smiles>

Benzyl propionate (67) ${ }^{10}$ : Colorless oil, $59 \mathrm{mg}, 90 \%$ yield, $\mathrm{R}_{\mathrm{f}}=0.2(\mathrm{PE} / \mathrm{EtOAc} 20 / 1) .{ }^{\mathbf{1}} \mathbf{H}$ NMR $\left(400 \mathrm{MHz}, \mathrm{CDCl}_{3}\right) \delta 7.41-7.27(\mathrm{~m}, 5 \mathrm{H}), 5.11(\mathrm{~s}, 2 \mathrm{H}), 2.37(\mathrm{q}, \mathrm{J}=7.5 \mathrm{~Hz}, 2 \mathrm{H}), 1.16(\mathrm{t}, \mathrm{J}=7.6 \mathrm{~Hz}$, $3 \mathrm{H}) .{ }^{13} \mathrm{CNMR}\left(100 \mathrm{MHz}, \mathrm{CDCl}_{3}\right) \delta 174.3,136.2,128.6,128.2,66.2,27.6,9.1$.

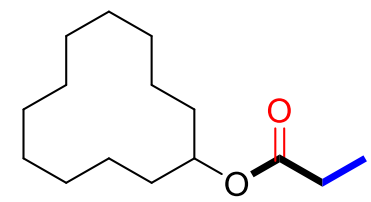

Cyclododecyl propionate (68) ${ }^{11}$ : Colorless oil, $92 \mathrm{mg}, 96 \%$ yield, $\mathrm{R}_{\mathrm{f}}=0.2(\mathrm{PE} / \mathrm{EtOAc} 20 / 1) .{ }^{1} \mathbf{H}$ NMR $\left(400 \mathrm{MHz}, \mathrm{CDCl}_{3}\right) \delta 5.02(\mathrm{t}, \mathrm{J}=6.2 \mathrm{~Hz}, 1 \mathrm{H}), 2.29$ (q, J = $\left.7.5 \mathrm{~Hz}, 2 \mathrm{H}\right), 1.76-1.64(\mathrm{~m}, 2 \mathrm{H})$, $1.56-1.42(\mathrm{~m}, 4 \mathrm{H}), 1.42-1.29(\mathrm{~m}, 16 \mathrm{H}), 1.13(\mathrm{t}, \mathrm{J}=7.5 \mathrm{~Hz}, 3 \mathrm{H}) .{ }^{13} \mathbf{C ~ N M R}\left(100 \mathrm{MHz}, \mathrm{CDCl}_{3}\right) \delta$ 174.1,71.9,29.1,27.9,24.1,23.8,23.4,23.2, 20.9,9.2.

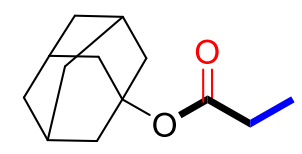

(5S,10R,13S,14S,17S)-4,4,10,13,14-pentamethyl-17-((S)-6-methylhept-5-en-2-yl)-2,3,4,5,6,7, $10,11,12,13,14,15,16,17$-tetradecahydro-1H-cyclopenta[a]phenanthren-3-yl propionate (69) $^{12}$ : Colorless oil, $67 \mathrm{mg}, 81 \%$ yield, $\mathrm{R}_{\mathrm{f}}=0.2(\mathrm{PE} / \mathrm{EtOAc} 20 / 1) .{ }^{1} \mathbf{H} \mathbf{~ N M R}\left(700 \mathrm{MHz}, \mathrm{CDCl}_{3}\right)$ $\delta 2.23(\mathrm{q}, \mathrm{J}=7.6 \mathrm{~Hz}, 2 \mathrm{H}), 2.16(\mathrm{~s}, 3 \mathrm{H}), 2.11(\mathrm{~s}, 6 \mathrm{H}), 1.71-1.60(\mathrm{~m}, 6 \mathrm{H}), 1.08(\mathrm{t}, \mathrm{J}=7.6 \mathrm{~Hz}, 3 \mathrm{H}) .{ }^{13} \mathrm{C}$ NMR $\left(176 \mathrm{MHz}, \mathrm{CDCl}_{3}\right) \delta 173.7,80.0,41.4,36.2,30.8,28.9,9.2$.

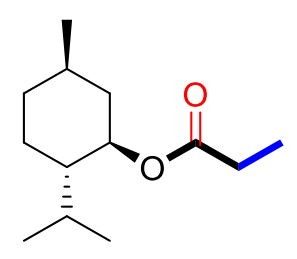

(1R,2S,5R)-2-isopropyl-5-methylcyclohexyl propionate (70) ${ }^{13}$ : Colorless oil, $80 \mathrm{mg}$, 94\% yield, $\mathrm{R}_{\mathrm{f}}=0.4(\mathrm{PE} / \mathrm{EtOAc} 20 / 1) .{ }^{1} \mathbf{H}$ NMR $\left(700 \mathrm{MHz}, \mathrm{CDCl}_{3}\right) \delta 4.68(\mathrm{td}, \mathrm{J}=10.9,4.4 \mathrm{~Hz}, 1 \mathrm{H})$, $2.30(\mathrm{qd}, \mathrm{J}=7.7,2.5 \mathrm{~Hz}, 2 \mathrm{H}), 1.98(\mathrm{dtd}, \mathrm{J}=12.1,4.0,1.9 \mathrm{~Hz}, 1 \mathrm{H}), 1.86(\mathrm{pd}, \mathrm{J}=7.0,2.7 \mathrm{~Hz}, 1 \mathrm{H})$, 1.68 (ddq, J = 13.2, 10.1, 3.4, 2.9 Hz, 2H), 1.49 (tdp, J = 12.7, 6.6, $3.1 \mathrm{~Hz}, 1 \mathrm{H}$ ), 1.37 (ddt, J = 14.2, 11.1, $3.2 \mathrm{~Hz}, 1 \mathrm{H}), 1.14(\mathrm{t}, \mathrm{J}=7.6 \mathrm{~Hz}, 3 \mathrm{H}), 1.10-1.02(\mathrm{~m}, 1 \mathrm{H}), 0.96(\mathrm{q}, \mathrm{J}=11.8 \mathrm{~Hz}, 1 \mathrm{H}), 0.92-0.84$ $(\mathrm{m}, 7 \mathrm{H}), 0.76(\mathrm{~d}, \mathrm{~J}=7.0 \mathrm{~Hz}, 3 \mathrm{H}) .{ }^{13} \mathbf{C} \mathbf{~ N M R}\left(176 \mathrm{MHz}, \mathrm{CDCl}_{3}\right) \delta 174.1,73.9,47.1,41.0,34.3,31.4$, 28.0,26.3, 23.5, 22.0, 20.8, 16.4,9.3. 


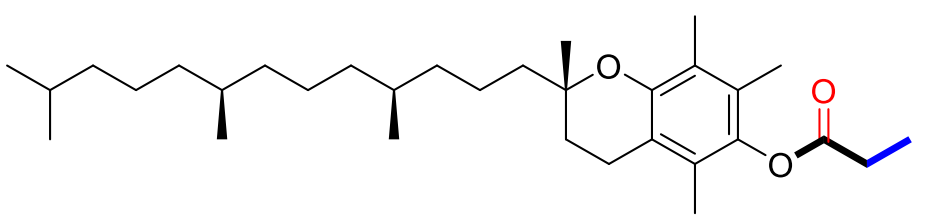

(S)-2,5,7,8-tetramethyl-2-((4S,8S)-4,8,12-trimethyltridecyl)chroman-6-yl propionate $(\mathbf{7 1})^{14}$ : Colorless oil, $181 \mathrm{mg}, 93 \%$ yield, $\mathrm{R}_{\mathrm{f}}=0.4(\mathrm{PE} / \mathrm{EtOAc} 20 / 1) .{ }^{1} \mathbf{H} \mathbf{N M R}\left(400 \mathrm{MHz}, \mathrm{CDCl}_{3}\right) \delta 2.67$ $-2.54(\mathrm{~m}, 4 \mathrm{H}), 2.09(\mathrm{~s}, 3 \mathrm{H}), 2.01(\mathrm{~s}, 3 \mathrm{H}), 1.96(\mathrm{~s}, 3 \mathrm{H}), 1.87-1.69(\mathrm{~m}, 2 \mathrm{H}), 1.38$ (ddd, J = 11.1, 7.0, $3.8 \mathrm{~Hz}, 7 \mathrm{H}), 1.35-1.19(\mathrm{~m}, 14 \mathrm{H}), 1.17-1.02(\mathrm{~m}, 6 \mathrm{H}), 0.91-0.80(\mathrm{~m}, 12 \mathrm{H}) .{ }^{13} \mathbf{C}$ NMR $(100 \mathrm{MHz}$, $\left.\mathrm{CDCl}_{3}\right) \delta 173.0,149.4,140.51,126.7,124.9,123.0,117.4,75.0,39.4,37.5,37.5,37.5,37.4,32.8$, $32.7,28.0,27.6,24.9,24.5,22.8,22.7,21.1,20.7,19.8,19.7,19.7,19.7,19.7,13.0,12.1,11.9,9.5$.<smiles>CCC(=O)OC/C=C(/C)CCC=C(C)C</smiles>

(Z)-3,7-dimethylocta-2,6-dien-1-yl propionate (72) ${ }^{15}$ : Colorless oil, $79 \mathrm{mg}, 94 \%$ yield, $\mathrm{R}_{\mathrm{f}}=0.2$ (PE/EtOAc 20/1). ${ }^{1} \mathbf{H}$ NMR (400 MHz, $\left.\mathrm{CDCl}_{3}\right) \delta 5.36(\mathrm{t}, \mathrm{J}=7.4 \mathrm{~Hz}, 1 \mathrm{H}), 5.10(\mathrm{t}, \mathrm{J}=6.6 \mathrm{~Hz}, 1 \mathrm{H})$, 4.57 (d, J = 7.2 Hz, 2H), 2.32 (q, J = 7.5 Hz, 2H), $2.16-2.04$ (m, 4H), 1.77 (s, 3H), 1.68 (s, 3H), 1.60 $(\mathrm{s}, 3 \mathrm{H}), 1.14$ (t, J = 7.6 Hz, 3H). ${ }^{13} \mathbf{C}$ NMR $\left(100 \mathrm{MHz}, \mathrm{CDCl}_{3}\right) \delta 174.4,142.4,132.1,123.6,119.3$, $60.9,32.2,27.6,26.6,25.6,23.5,17.6,9.1$.

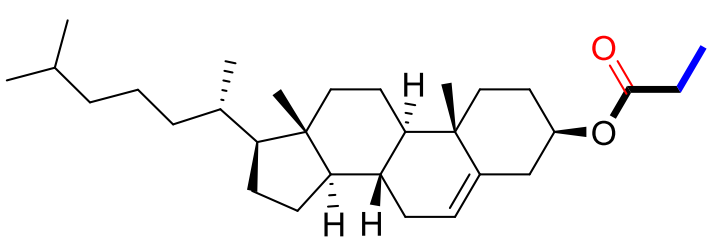

(3R,8R,9R,10S,13S,14R,17S)-10,13-dimethyl-17-((S)-6-methylheptan-2-yl)-2,3,4,7,8,9,10,11, $\mathbf{1 2 , 1 3 , 1 4 , 1 5 , 1 6 , 1 7 - t e t r a d e c a h y d r o - 1 H}$-cyclopenta[a]phenanthren-3-yl propionate $(\mathbf{7 3})^{16}$ : White solid, $139 \mathrm{mg}, 79 \%$ yield, $\mathrm{R}_{\mathrm{f}}=0.2(\mathrm{PE} / \mathrm{EtOAc} 20 / 1) .{ }^{1} \mathbf{H} \mathbf{~ N M R}\left(400 \mathrm{MHz}, \mathrm{CDCl}_{3}\right) \delta 5.29$ $(\mathrm{d}, \mathrm{J}=4.9 \mathrm{~Hz}, 1 \mathrm{H}), 4.61-4.48(\mathrm{~m}, 1 \mathrm{H}), 2.22(\mathrm{q}, \mathrm{J}=7.8 \mathrm{~Hz}, 4 \mathrm{H}), 1.98-1.85(\mathrm{~m}, 2 \mathrm{H}), 1.84-1.69(\mathrm{~m}$, $3 \mathrm{H}), 1.54-1.35(\mathrm{~m}, 7 \mathrm{H}), 1.32-1.17(\mathrm{~m}, 4 \mathrm{H}), 1.14-0.98(\mathrm{~m}, 10 \mathrm{H}), 0.98-0.88(\mathrm{~m}, 6 \mathrm{H}), 0.84(\mathrm{~d}, \mathrm{~J}=$ $6.4 \mathrm{~Hz}, 3 \mathrm{H}), 0.80$ (s, 3H), 0.78 (s, 3H), 0.61 (s, 3H). ${ }^{13} \mathbf{C}$ NMR (100 MHz, CDCl $) \delta$ 173.8, 139.7, $122.6,73.7,56.7,56.2,50.0,42.3,39.8,39.5,38.2,37.0,36.6,36.2,35.8,31.9,31.9,28.3,28.0,27.9$, $27.8,24.3,23.9,22.8,22.6,21.1,19.3,18.7,11.9,9.2$.<smiles>CCC(=O)N(C(C)=O)c1ccccc1</smiles>

$N$-acetyl- $N$-phenylpropionamide (74) ${ }^{17}$ : Colorless oil, $66 \mathrm{mg}, 86 \%$ yield, $\mathrm{R}_{\mathrm{f}}=0.2(\mathrm{PE} / \mathrm{EtOAc}$ 10/1). ${ }^{1} \mathbf{H}$ NMR $\left(700 \mathrm{MHz}, \mathrm{CDCl}_{3}\right) \delta 7.46(\mathrm{t}, \mathrm{J}=7.6 \mathrm{~Hz}, 2 \mathrm{H}), 7.41(\mathrm{t}, \mathrm{J}=7.4 \mathrm{~Hz}, 1 \mathrm{H}), 7.14(\mathrm{~d}, \mathrm{~J}=$ $7.5 \mathrm{~Hz}, 2 \mathrm{H}), 2.49$ (q, J = 7.3 Hz, 2H), 2.36 (s, 3H), 1.09 (t, J = 7.3 Hz, 3H). ${ }^{13} \mathrm{C}$ NMR (176 MHz, $\left.\mathrm{CDCl}_{3}\right) \delta 176.6,173.1,139.2,129.8,128.8,128.8,32.0,27.2,8.9$. 


\section{References}

1. Lurie, I. S.; Berrier, A. L.; Casale, J. F.; lio, R.; Bozenko, J. S. Profiling of Illicit Fentanyl using UHPLC-MS/MS. Forensic Sci. Int. 2012, 220(1), 191-196.

2. Tong, H.-R.; Zheng, W.; Lv, X.; He, G.; Liu, P.; Chen, G. Asymmetric Synthesis of $\beta$-Lactam via Palladium-Catalyzed Enantioselective Intramolecular C(sp3)-H Amidation. ACS Catal. 2020, 10 (1), 114-120.

3. Li, J.; Subramaniam, K.; Smith, D.; Qiao, J. X.; Li, J. J.; Qian-Cutrone, J.; Kadow, J. F.; Vite, G. D.; Chen, B.-C. AlMe3-Promoted Formation of Amides from Acids and Amines. Org. Lett. 2012, 14 (1), 214-217.

4. Castanheiro, T.; Suffert, J.; Gulea, M.; Donnard, M. Aerobic Copper-Mediated Domino Three-Component Approach to 2-Aminobenzothiazole Derivatives. Org. Lett. 2016, 18 (11), 2588-2591.

5. Mayer, B. P.; DeHope, A. J.; Mew, D. A.; Spackman, P. E.; Williams, A. M. Chemical Attribution of Fentanyl Using Multivariate Statistical Analysis of Orthogonal Mass Spectral Data. Anal. Chem. 2016, $88(8), 4303-4310$.

6. Ben Halima, T.; Zhang, W.; Yalaoui, I.; Hong, X.; Yang, Y.-F.; Houk, K. N.; Newman, S. G. Palladium-Catalyzed Suzuki-Miyaura Coupling of Aryl Esters. J. Am. Chem. Soc. 2017, 139 (3), 1311-1318.

7. Iranpoor, N.; Firouzabadi, H.; Khalili, D.; Motevalli, S. Easily Prepared Azopyridines As Potent and Recyclable Reagents for Facile Esterification Reactions. An Efficient Modified Mitsunobu Reaction. J. Org. Chem. 2008, 73 (13), 4882-4887.

8. Damiens, A.; Alebrahim, M. T.; Léonard, E.; Fayeulle, A.; Furman, C.; Hilbert, J.-L.; Siah, A.; Billamboz, M. Sesamol-based Terpenoids as Promising Bio-sourced Crop Protection Compounds Against the Wheat Pathogen Zymoseptoria Tritici. Pest Manag. Sci. 2021, 77(5), 2403-2414.

9. Closson, W. D.; Orenski, P. J.; Goldschmidt, B. M. Effect of Conformation on the Electronic Absorption Spectrum of the Carboxylate Group. J. Org. Chem. 1967, 32 (10), 3160-3163.

10. Li, G.; Garcia-Borràs, M.; Fürst, M. J. L. J.; Ilie, A.; Fraaije, M. W.; Houk, K. N.; Reetz, M. T. Overriding Traditional Electronic Effects in Biocatalytic Baeyer-Villiger Reactions by Directed Evolution. J. Am. Chem. Soc. 2018, 140(33), 10464-10472.

11. Michel, D.; Schlosser, M. Odor-Structure Relationships Using Fluorine as a Probe. Tetrahedron 2000, 56 (25), 4253-4260.

12. Hatano, M.; Furuya, Y.; Shimmura, T.; Moriyama, K.; Kamiya, S.; Maki, T.; Ishihara, K. Ligand-Assisted Rate Acceleration in Lanthanum(III) Isopropoxide Catalyzed Transesterification of Carboxylic Esters. Org. Lett. 2011, 13(3), 426-429.

13. Birman, V. B.; Li, X. Homobenzotetramisole: An Effective Catalyst for Kinetic Resolution of Aryl-Cycloalkanols. Org. Lett. 2008, 10(6), 1115-1118.

14. Woollard, D. C.; Bensch, A.; Indyk, H.; McMahon, A. Determination of Vitamin A and Vitamin E esters in Infant Formulae and Fortified Milk Powders by HPLC: Use of Internal Standardisation. Food Chem. 2016, 197, 457-465.

15. Zhang, Q.; Catti, L.; Pleiss, J.; Tiefenbacher, K. Terpene Cyclizations inside a Supramolecular Catalyst: Leaving-Group-Controlled Product Selectivity and Mechanistic Studies. J. Am. Chem. Soc. 2017, 139(33), 11482-11492.

16. Frank, D. J.; Madrona, Y.; Ortiz de Montellano, P. R. Cholesterol Ester Oxidation by Mycobacterial Cytochrome P450*. J. Biol. Chem. 2014, 289 (44), 30417-30425. 
17. Kikukawa, K.; Kono, K.; Wada, F; Matsuda, T. Reaction of Arenediazonium Salts with Nitriles in the Presence of Sodium Carboxylates. A Convenient Synthesis of Unsymmetrical N-Aryl Acyclic Imides. B. Chem. Soc. Jpn 1982, 55 (11), 3671-3672. 


\section{NMR Spectra of the Products}

csk-2-101-9.11. fid

PROTON CDCI3 \{D:INMR400ldnl0604\} nmr-new 22
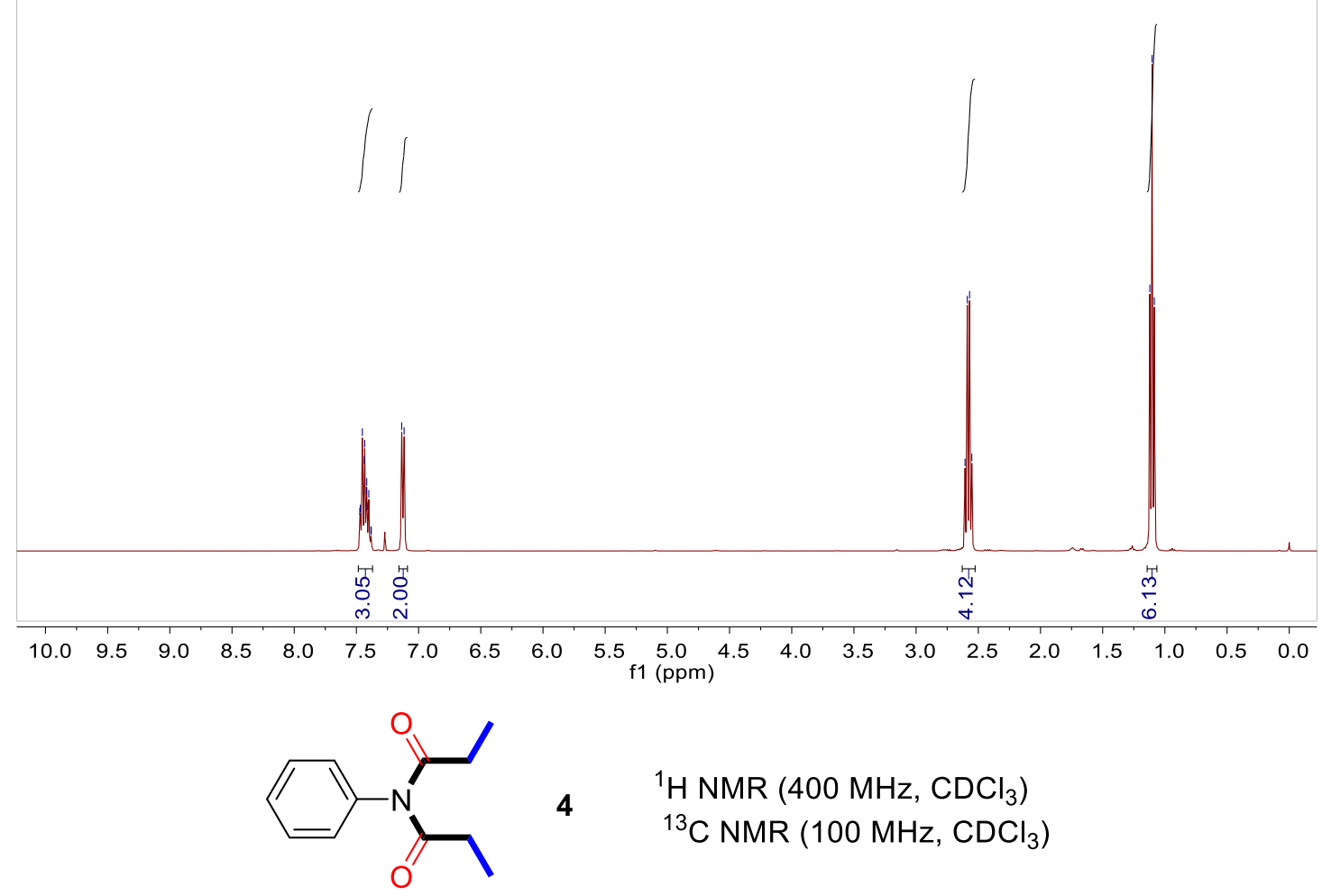

$4 \quad{ }^{1} \mathrm{H}$ NMR $\left(400 \mathrm{MHz}, \mathrm{CDCl}_{3}\right)$

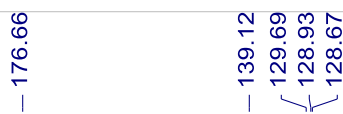

${ }^{13} \mathrm{C}$ NMR $\left(100 \mathrm{MHz}, \mathrm{CDCl}_{3}\right)$

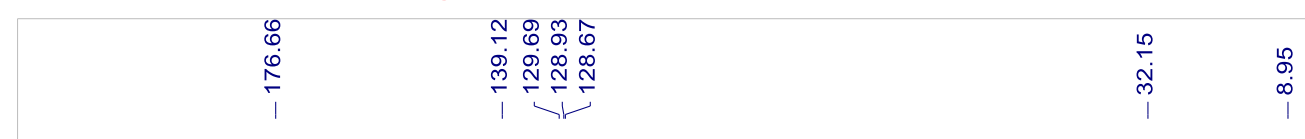

csk-2-101-9.12.fid

C13CPD CDCI3 \{D:INMR400ldnl0604\} nmr-new 22

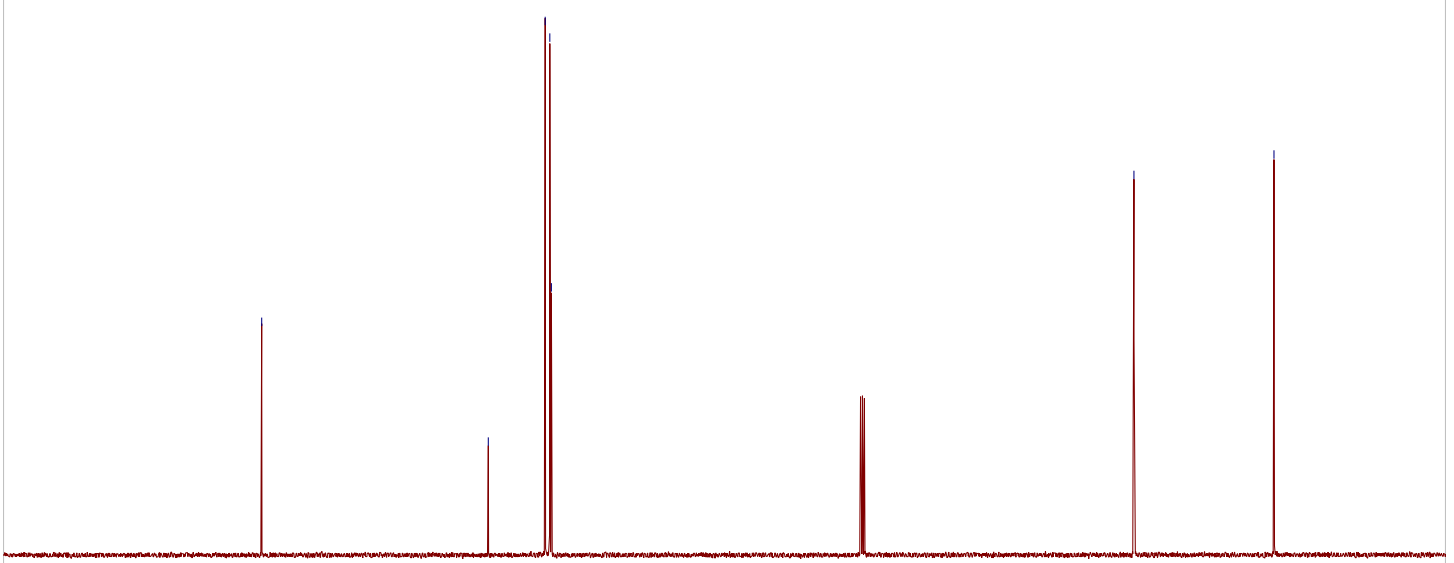

$\begin{array}{llllllllllllllllllllll}210 & 200 & 190 & 180 & 170 & 160 & 150 & 140 & 130 & 120 & 110 \\ \mathrm{f} 1 & 100 & 90 & 80 & 80 & 70 & 60 & 50 & 40 & 30 & 20 & 10 & 0 & -10\end{array}$ 
csk-2-101-4.10.fid

PROTON CDCI3 \{D:INMR400IDNL0604\} nmr-new 50

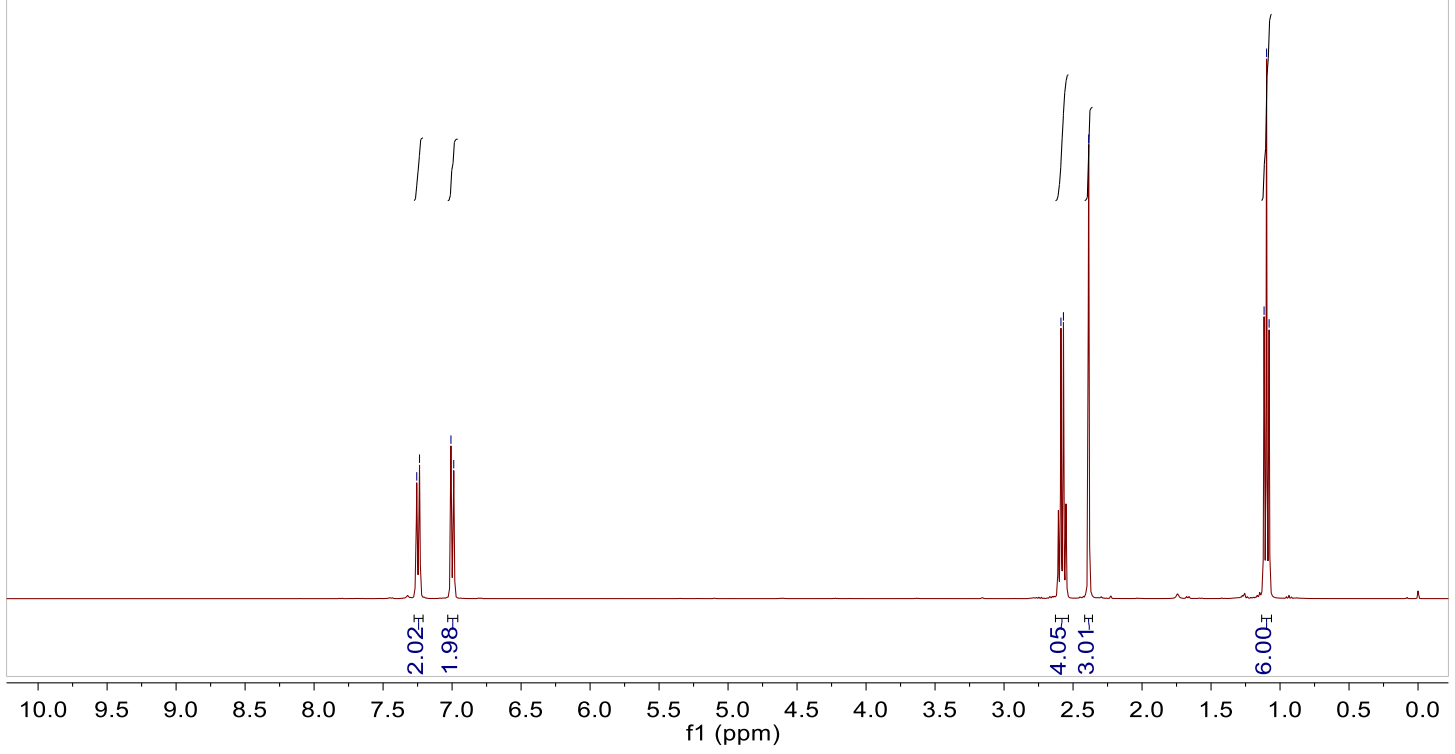

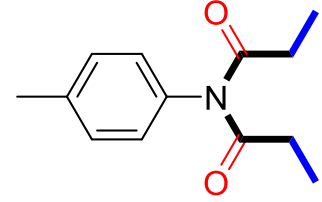

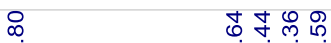

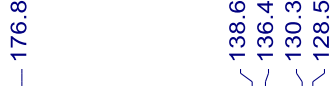

sk-2-101-4.11.fid

C13CPD CDCI3 \{D:INMR400IDNL0604\} nmr-new 50
${ }^{1} \mathrm{H}$ NMR $\left(400 \mathrm{MHz}, \mathrm{CDCl}_{3}\right)$

\section{$5 \quad{ }^{13} \mathrm{C} \mathrm{NMR}\left(100 \mathrm{MHz}, \mathrm{CDCl}_{3}\right)$}

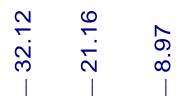

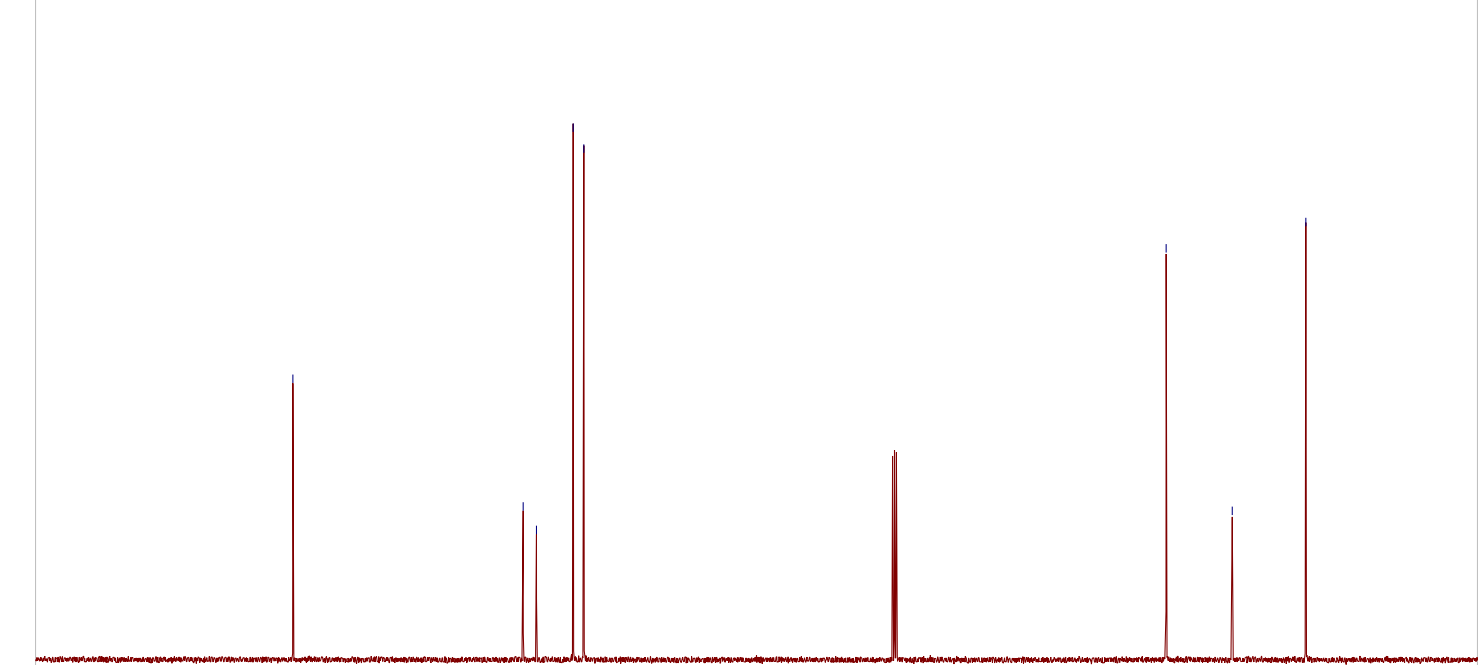

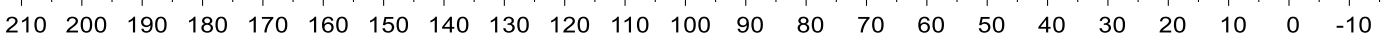
f1 (ppm) 
csk-3-5-8.10.fid

PROTON CDCI3 \{D:INMR400Idnl0604\} nmr-new 19
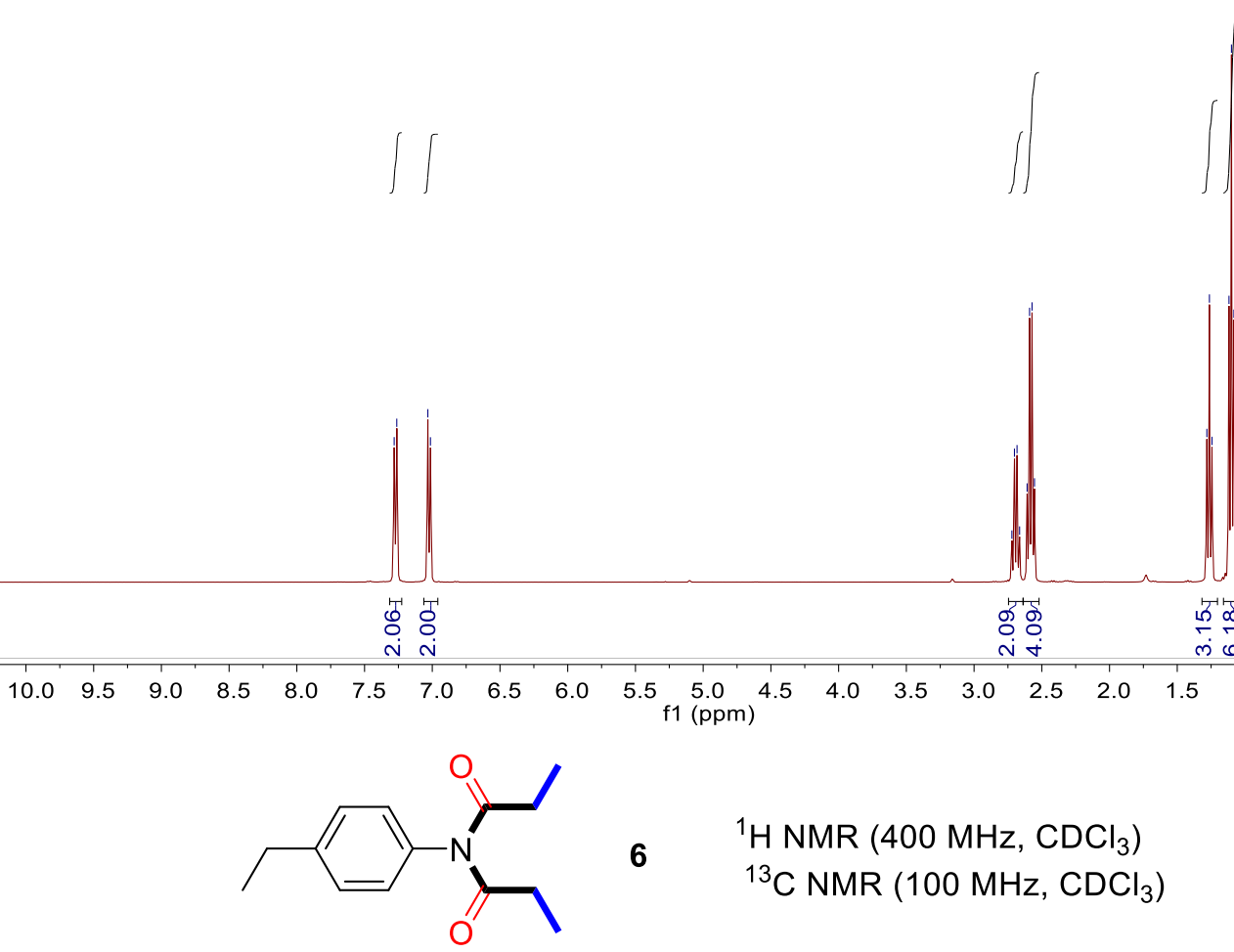

6

${ }^{1} \mathrm{H} \mathrm{NMR}\left(400 \mathrm{MHz}, \mathrm{CDCl}_{3}\right)$

${ }^{13} \mathrm{C}$ NMR $\left(100 \mathrm{MHz}, \mathrm{CDCl}_{3}\right)$

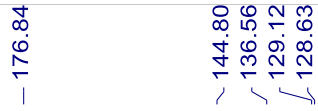

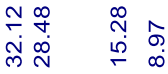

sk-3-5-8.11.fid

C13CPD CDCl3 \{D:INMR400ldnl0604\} nmr-new 19

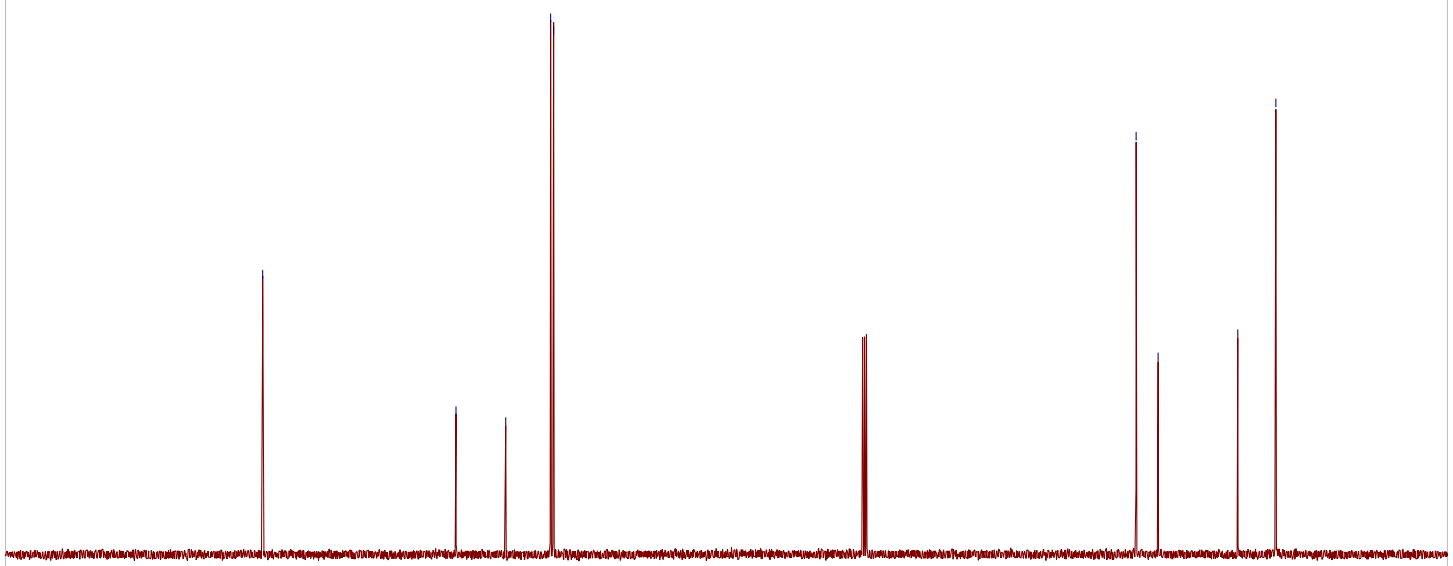

$\begin{array}{lllllllllllllllllllllll}210 & 200 & 190 & 180 & 170 & 160 & 150 & 140 & 130 & 120 & 110 & 100 & 90 & 80 & 70 & 60 & 50 & 40 & 30 & 20 & 10 & 0 & -10\end{array}$ 
csk-3-5-6.10.fid

PROTON CDCI3 \{D:INMR400ldnl0604\} nmr-new 8
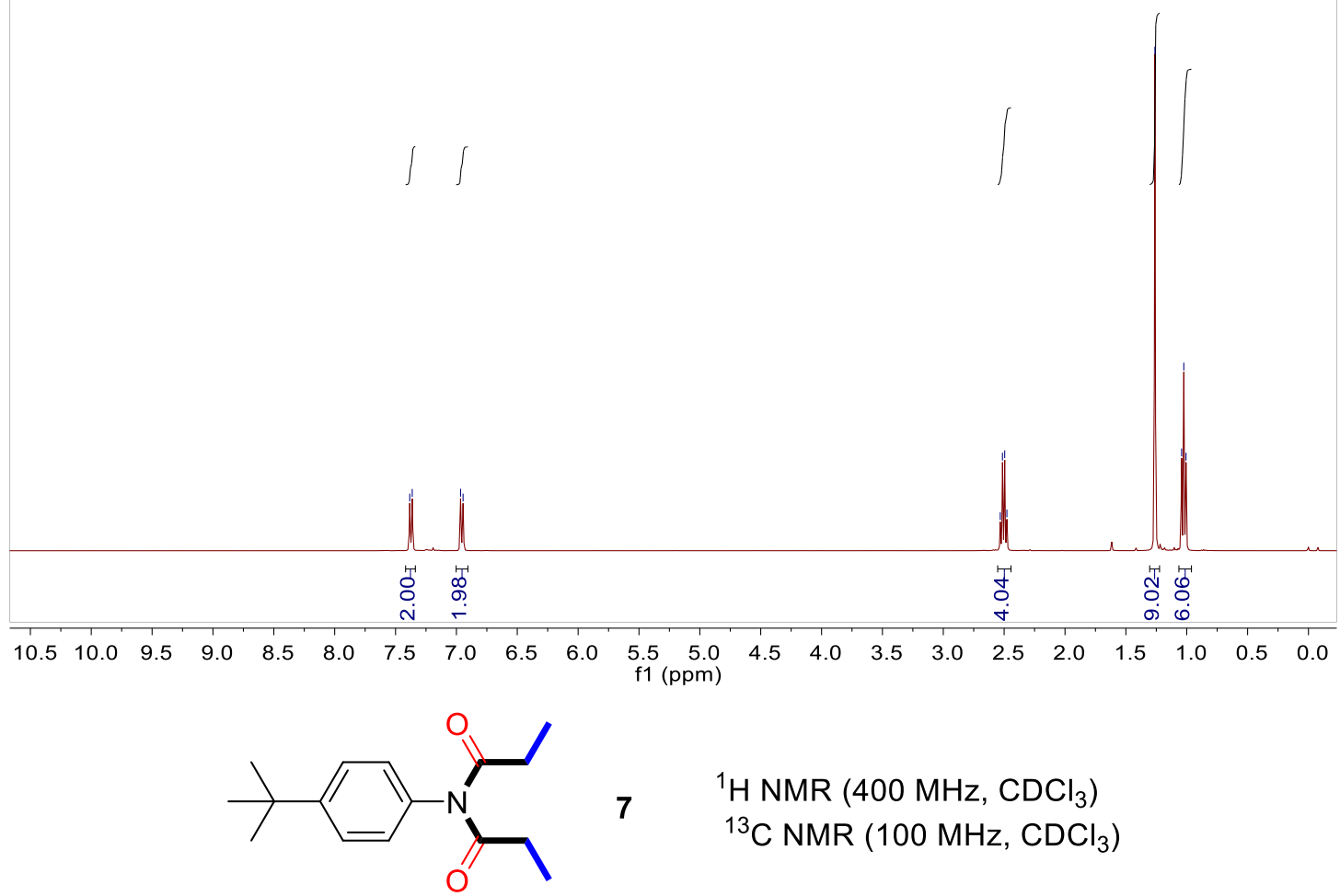

$7{ }^{1} \mathrm{H} \mathrm{NMR}\left(400 \mathrm{MHz}, \mathrm{CDCl}_{3}\right)$ ${ }^{13} \mathrm{C}$ NMR $\left(100 \mathrm{MHz}, \mathrm{CDCl}_{3}\right)$

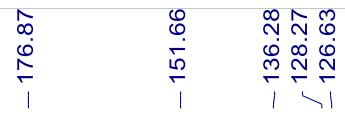

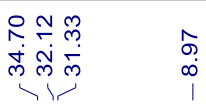

sk-3-5-6.11.fid

C13CPD CDCI3 \{D:INMR400ldn10604\} nmr-new 8

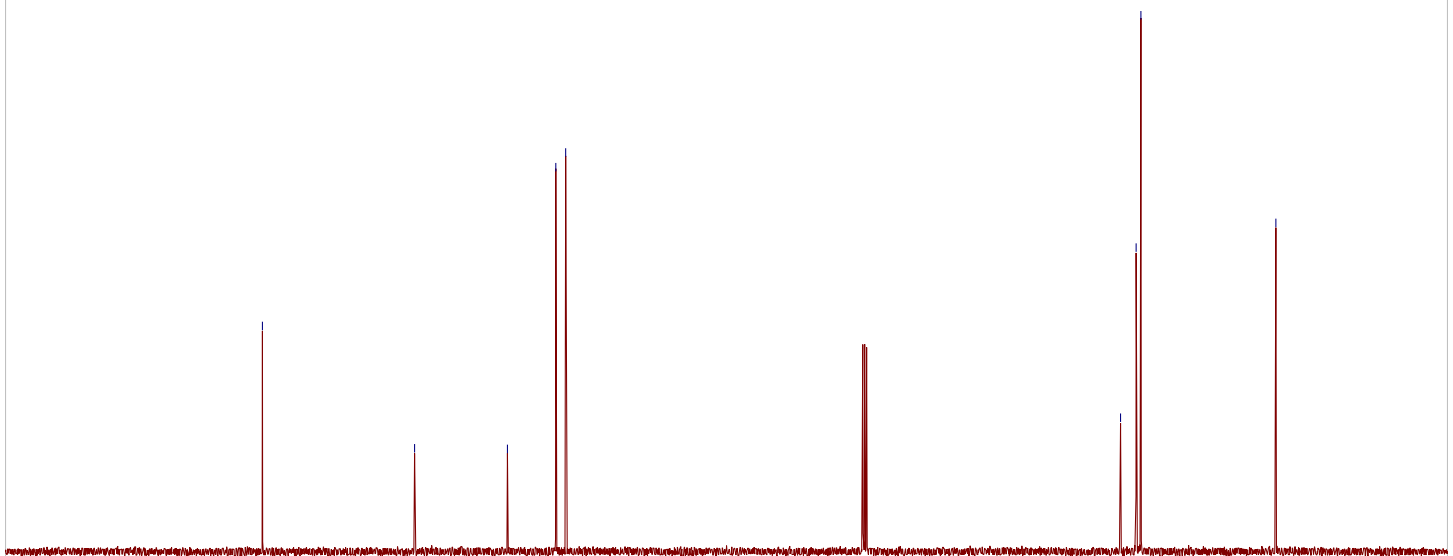

$\begin{array}{lllllllllllllllllllllll}210 & 200 & 190 & 180 & 170 & 160 & 150 & 140 & 130 & 120 & 110 & 100 & 90 & 80 & 70 & 60 & 50 & 40 & 30 & 20 & 10 & 0 & -10\end{array}$ 


\begin{tabular}{|c|c|c|}
\hline 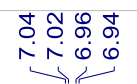 & $\begin{array}{c}m \\
\infty \\
\infty \\
1 \\
1\end{array}$ & 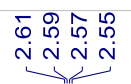 \\
\hline
\end{tabular}

csk-2-101-1.10.fid

PROTON CDCI3 \{D:INMR400IDNL0604\} nmr-new 47

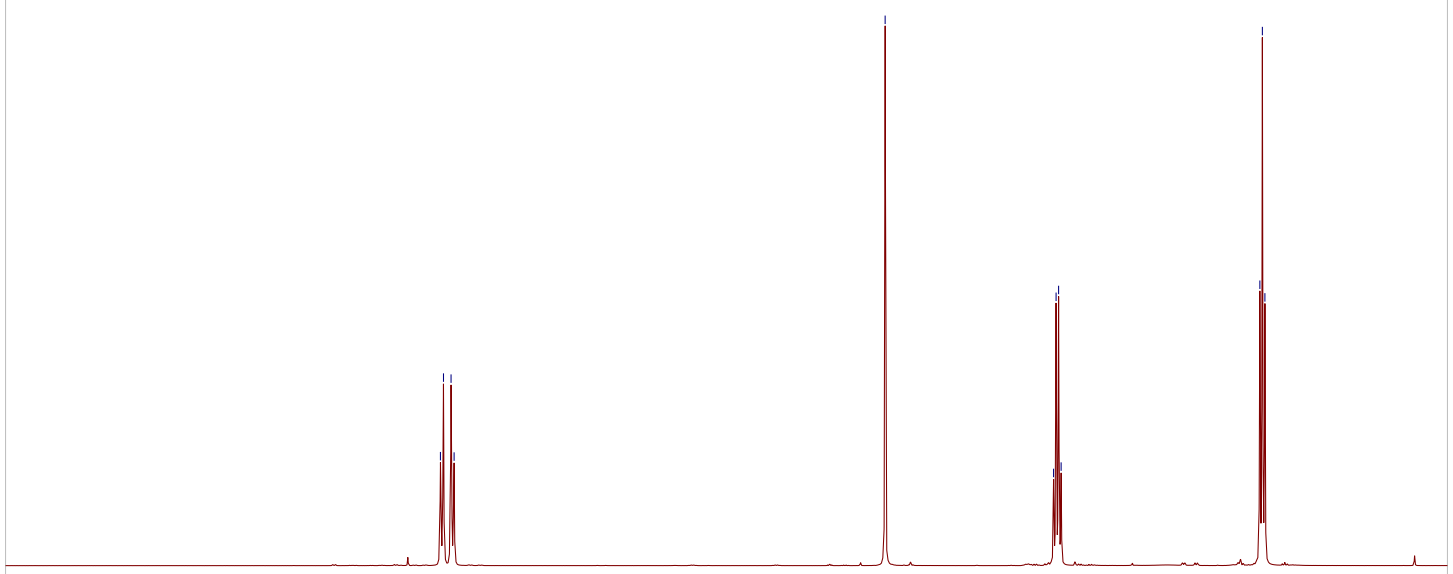

$\begin{array}{llllllllllllllllllllll}10.0 & 9.5 & 9.0 & 8.5 & 8.0 & 7.5 & 7.0 & 6.5 & 6.0 & 5.5 & \begin{array}{c}5.0 \\ (\mathrm{ppm})\end{array} & 4.5 & 4.0 & 3.5 & 3.0 & 2.5 & 2.0 & 1.5 & 1.0 & 0.5 & 0.0\end{array}$

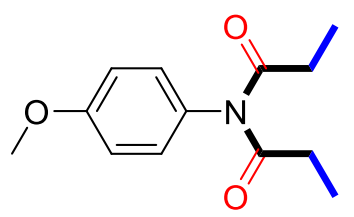

$8 \quad{ }^{1} \mathrm{H}$ NMR $\left(400 \mathrm{MHz}, \mathrm{CDCl}_{3}\right)$

${ }^{13} \mathrm{C} \mathrm{NMR}\left(100 \mathrm{MHz}, \mathrm{CDCl}_{3}\right)$

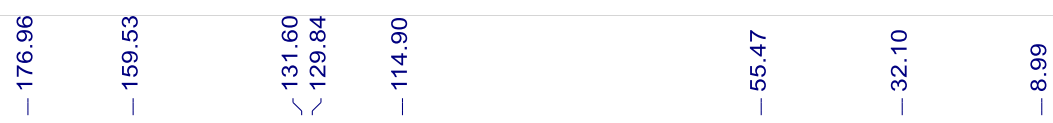

csk-2-101-1.11.fid

C13CPD CDCI3 \{D:INMR400IDNL0604\} nmr-new 47

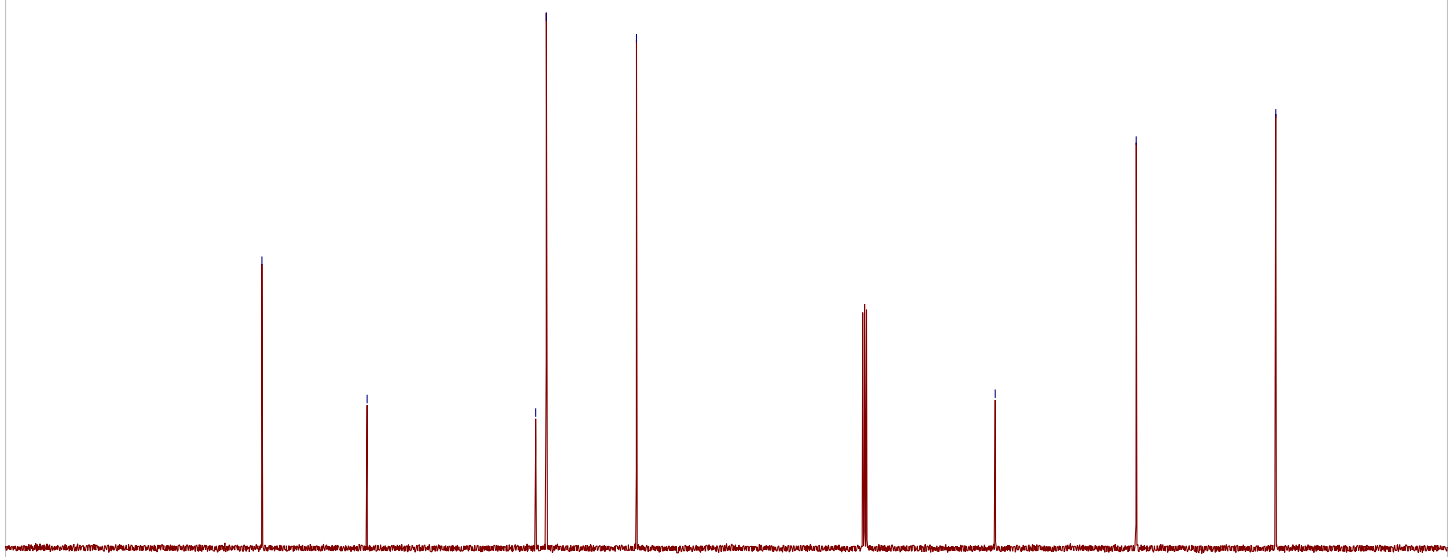

$\begin{array}{lllllllllllllllllllllll}210 & 200 & 190 & 180 & 170 & 160 & 150 & 140 & 130 & 120 & 110 & 100 & 90 & 80 & 70 & 60 & 50 & 40 & 30 & 20 & 10 & 0 & -10\end{array}$ f1 (ppm) 


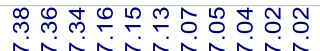 \\ ríñáñín}

ใั

in $\sin$

$\stackrel{m}{\leftarrow} \underset{\sim}{\leftarrow} \stackrel{i}{r}$

年

csk-3-19-5.10.fid

PROTON CDCI3 \{D:INMR400IDNL0604\} nmr-new 45
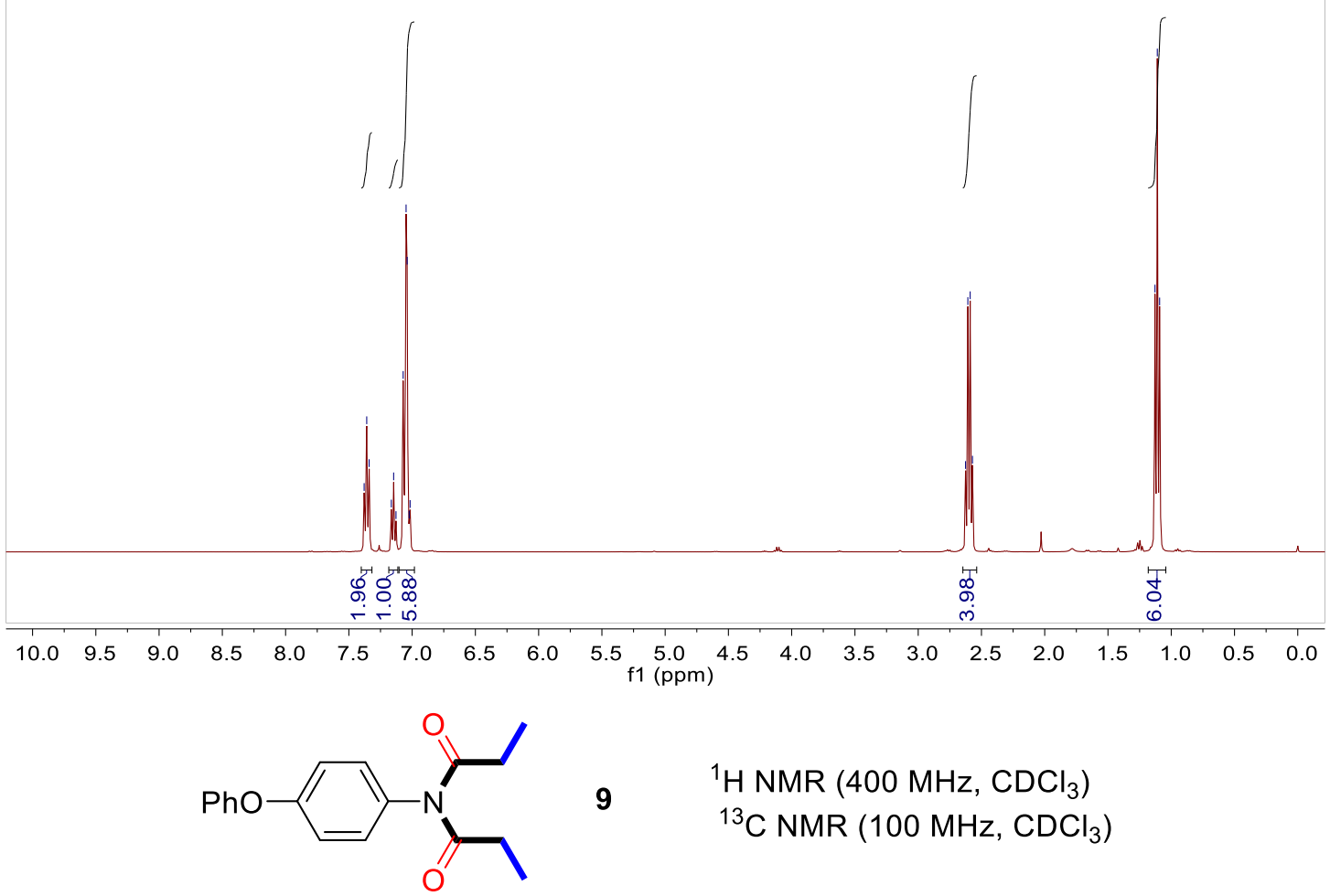

9

${ }^{1} \mathrm{H}$ NMR $\left(400 \mathrm{MHz}, \mathrm{CDCl}_{3}\right)$

${ }^{13} \mathrm{C} \mathrm{NMR}\left(100 \mathrm{MHz}, \mathrm{CDCl}_{3}\right)$

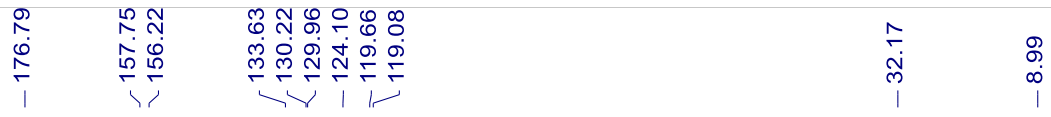

csk-3-19-5.11. fid

C13CPD CDCI3 \{D:INMR400IDNL0604\} nmr-new 45

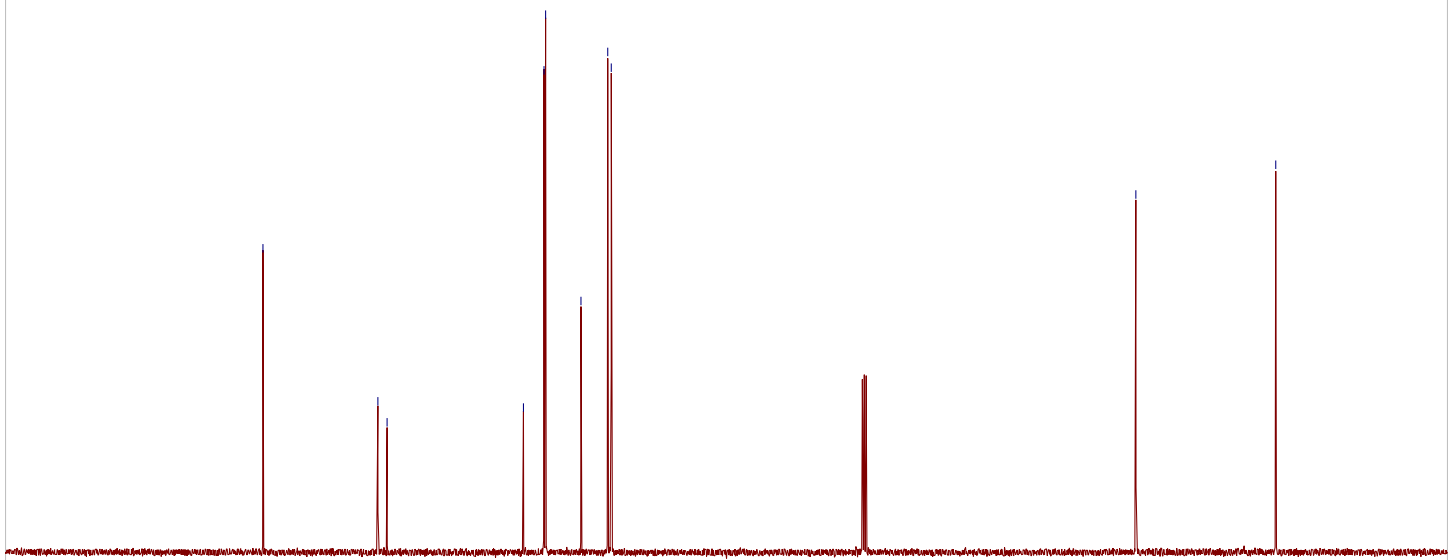

$\begin{array}{lllllllllllllllllllllll}210 & 200 & 190 & 180 & 170 & 160 & 150 & 140 & 130 & 120 & 110 & 100 & 90 & 80 & 70 & 60 & 50 & 40 & 30 & 20 & 10 & 0 & -10\end{array}$ 
csk-3-5-11.10.fid

PROTON CDCI3 \{D:INMR400Idnl0604\} nmr-new 20

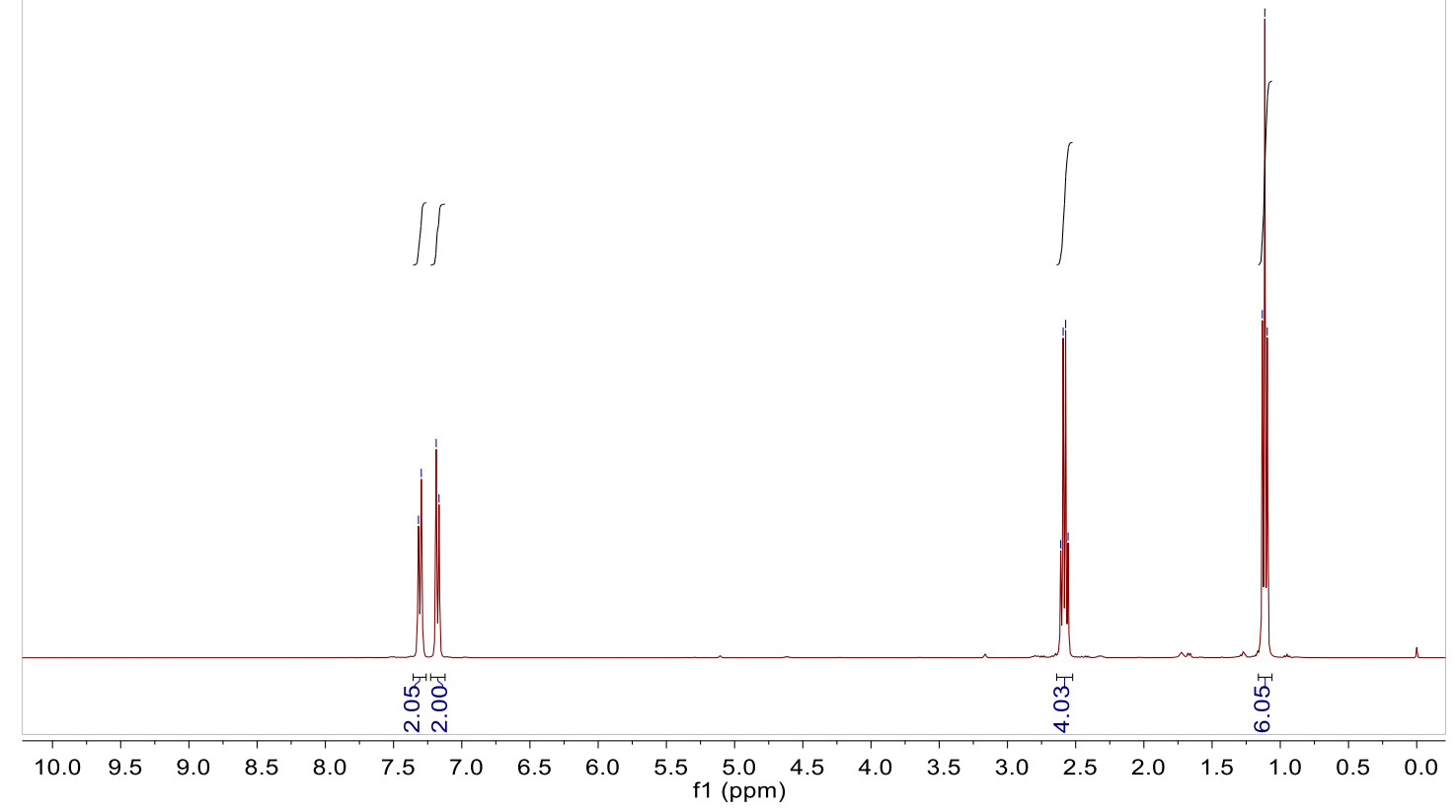<smiles>CCC(=O)N(C(=O)CC)c1ccc(OC(F)(F)F)cc1</smiles>

$10 \quad{ }^{1} \mathrm{H} \mathrm{NMR}\left(400 \mathrm{MHz}, \mathrm{CDCl}_{3}\right)$

${ }^{13} \mathrm{C} \mathrm{NMR}\left(100 \mathrm{MHz}, \mathrm{CDCl}_{3}\right)$

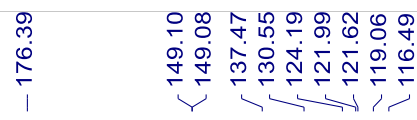

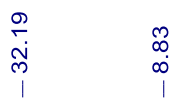

csk-3-5-11.11.fid

C13CPD CDCI3 \{D:INMR400ldnl0604\} nmr-new 20

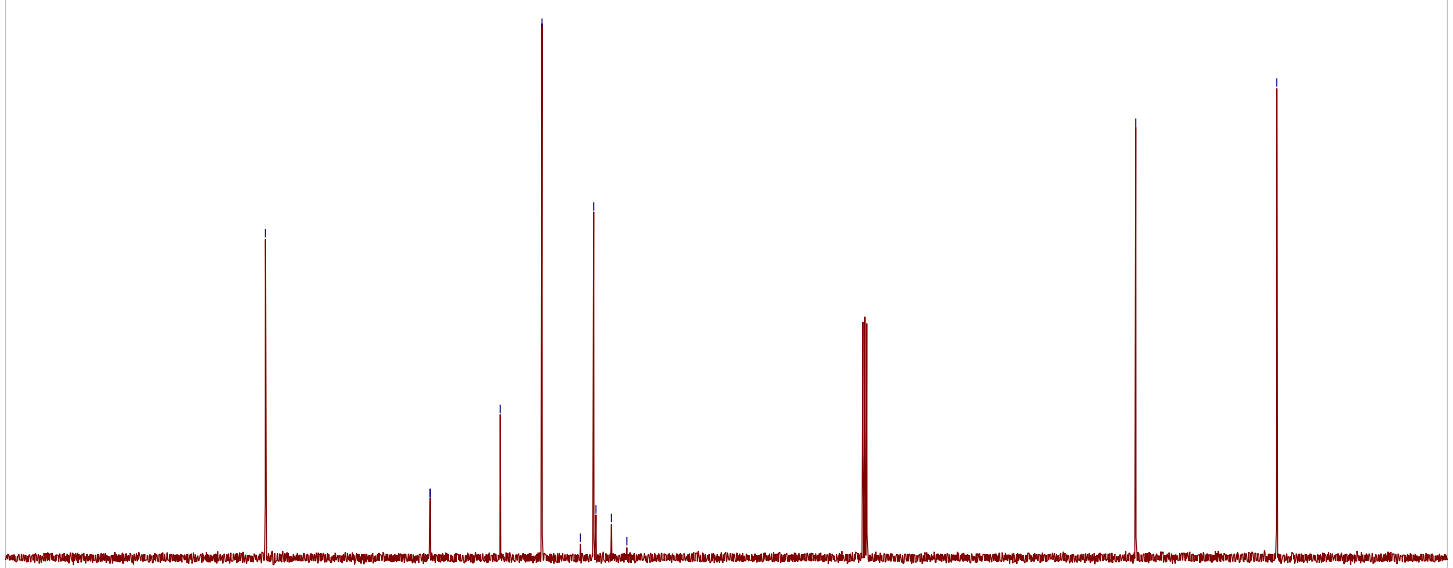

$\begin{array}{lllllllllllllllllllllll}210 & 200 & 190 & 180 & 170 & 160 & 150 & 140 & 130 & 120 & 110 & 100 & 90 & 80 & 70 & 60 & 50 & 40 & 30 & 20 & 10 & 0 & -10\end{array}$ 


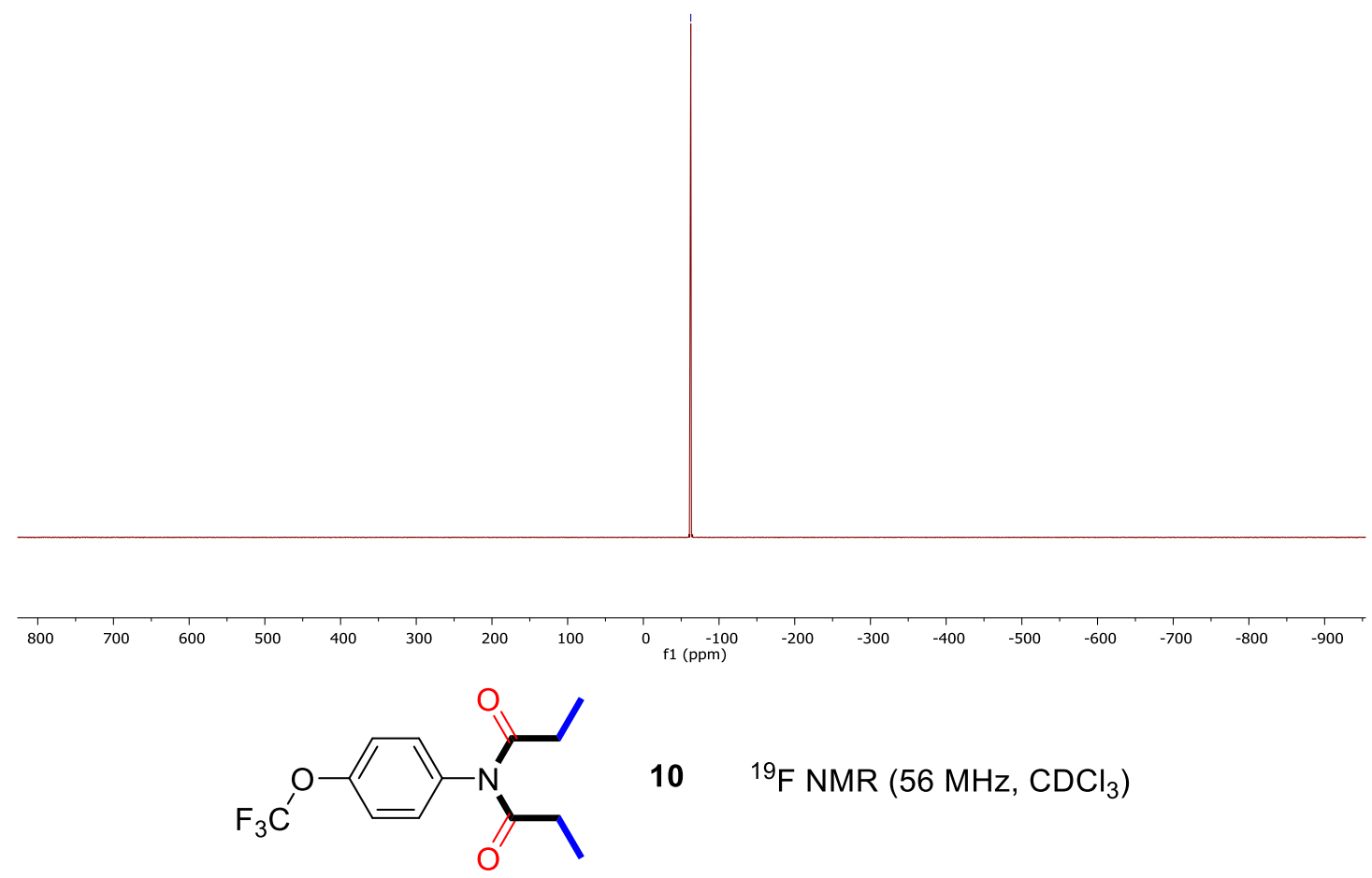


csk-3-12-11.10.fid

PROTON CDCI3 \{D:INMR400IDNL0604\} nmr-new 38

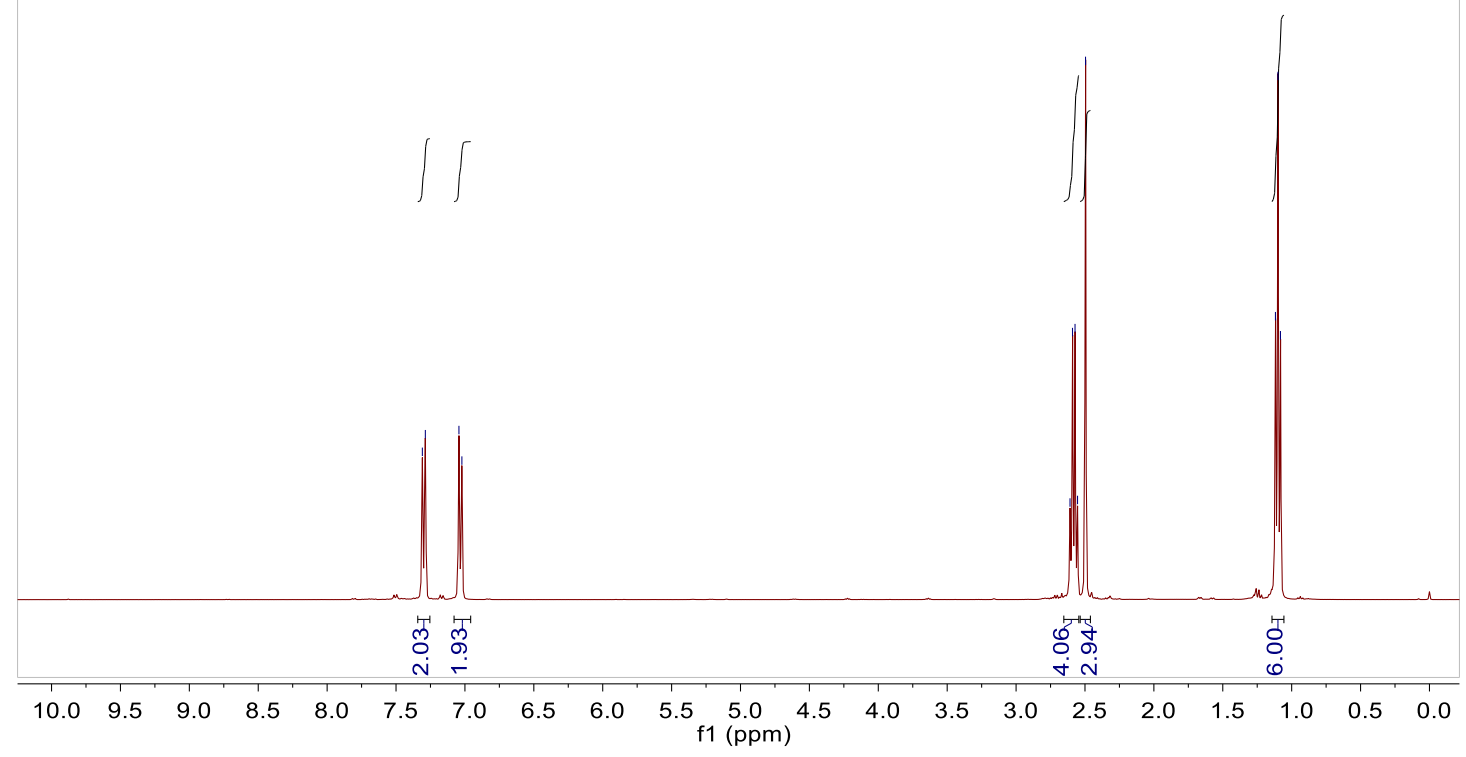

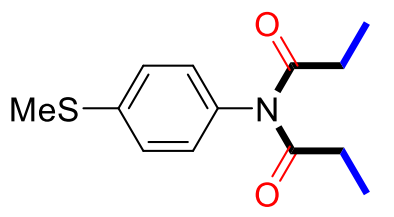

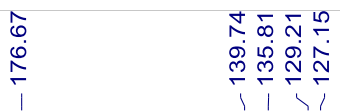

csk-3-12-11.11.fid

C13CPD CDCI3 \{D:INMR400IDNL0604\} nmr-new 38
11

${ }^{1} \mathrm{H}$ NMR (400 MHz, $\left.\mathrm{CDCl}_{3}\right)$

${ }^{13} \mathrm{C}$ NMR $\left(100 \mathrm{MHz}, \mathrm{CDCl}_{3}\right)$

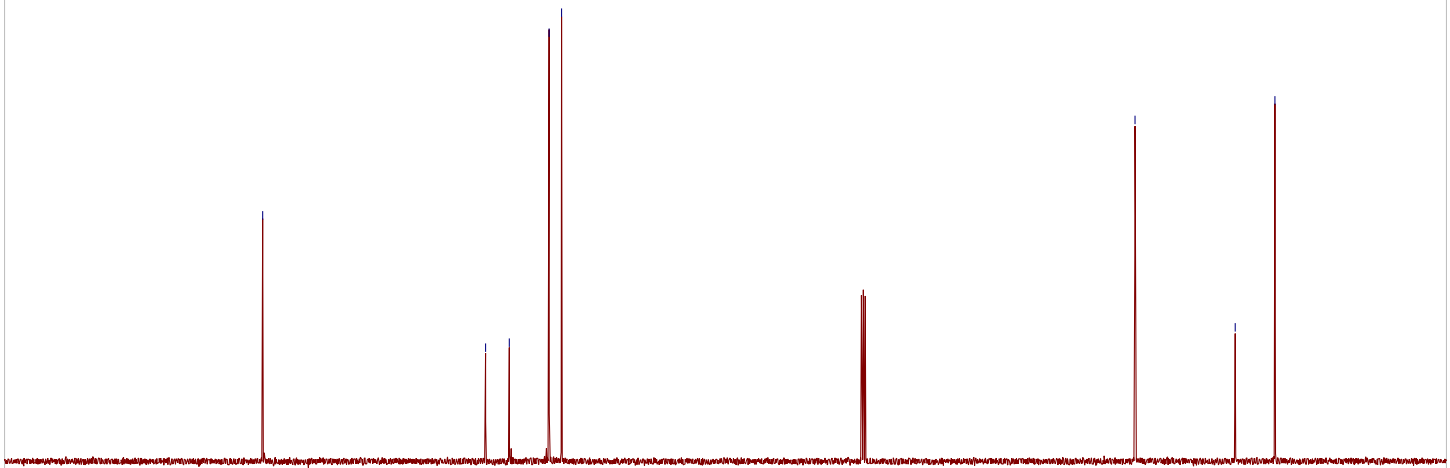

$\begin{array}{lllllllllllllllllllllll}210 & 200 & 190 & 180 & 170 & 160 & 150 & 140 & 130 & 120 & 110 & 100 & 90 & 80 & 70 & 60 & 50 & 40 & 30 & 20 & 10 & 0 & -10\end{array}$ 
csk-3-5-19.10.fid

PROTON CDCI3 \{D:INMR400Idnl0604\} nmr-new 35
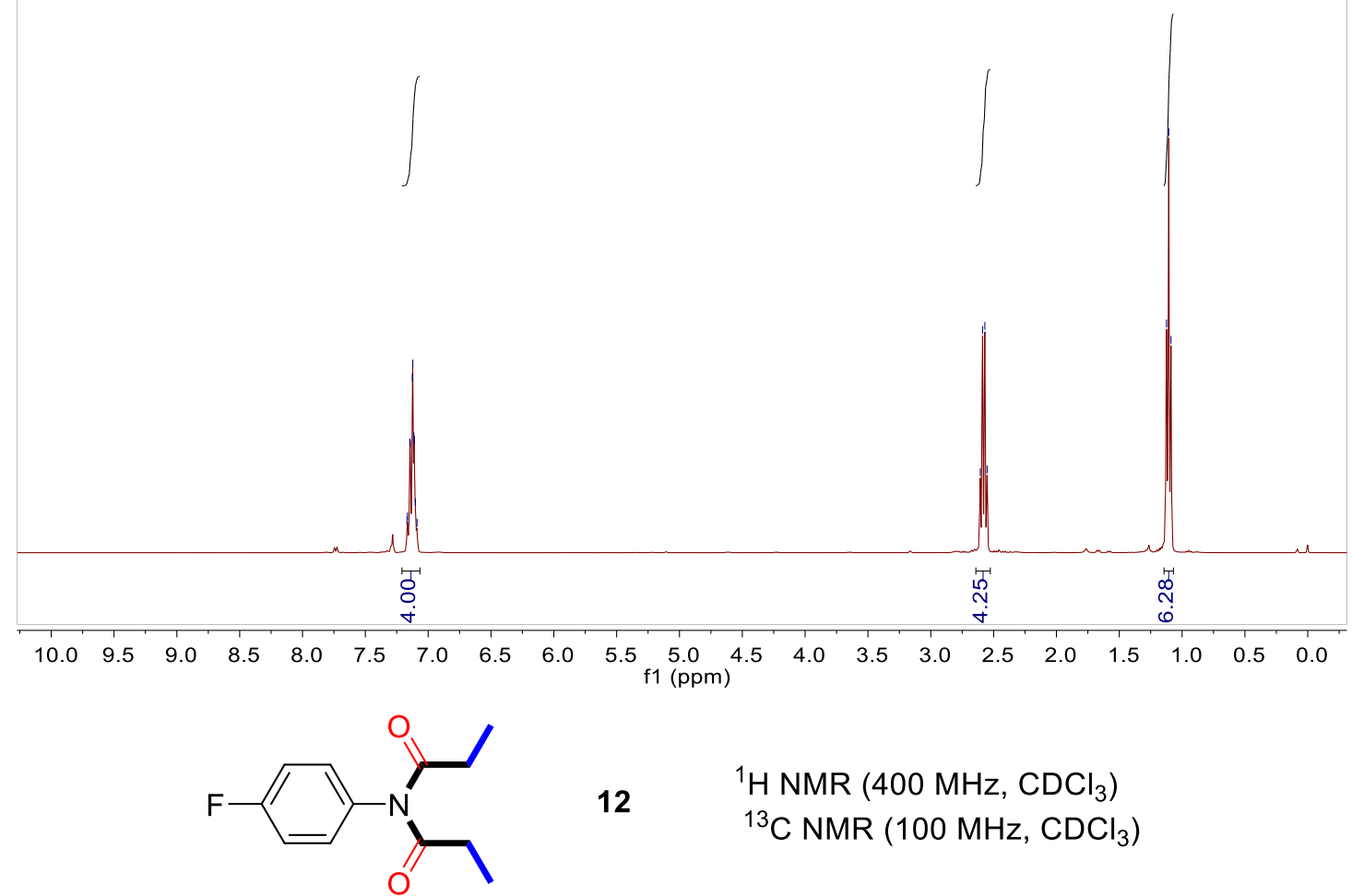

12

${ }^{1} \mathrm{H}$ NMR $\left(400 \mathrm{MHz}, \mathrm{CDCl}_{3}\right)$

${ }^{13} \mathrm{C}$ NMR $\left(100 \mathrm{MHz}, \mathrm{CDCl}_{3}\right)$

\begin{tabular}{|c|c|c|}
\hline 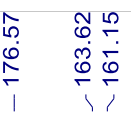 & 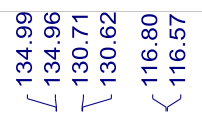 & $\stackrel{\circ}{\stackrel{\oplus}{i j}}$ \\
\hline
\end{tabular}

csk-3-5-19.11. fid

C13CPD CDCI3 \{D:INMR4001dn10604\} nmr-new 35

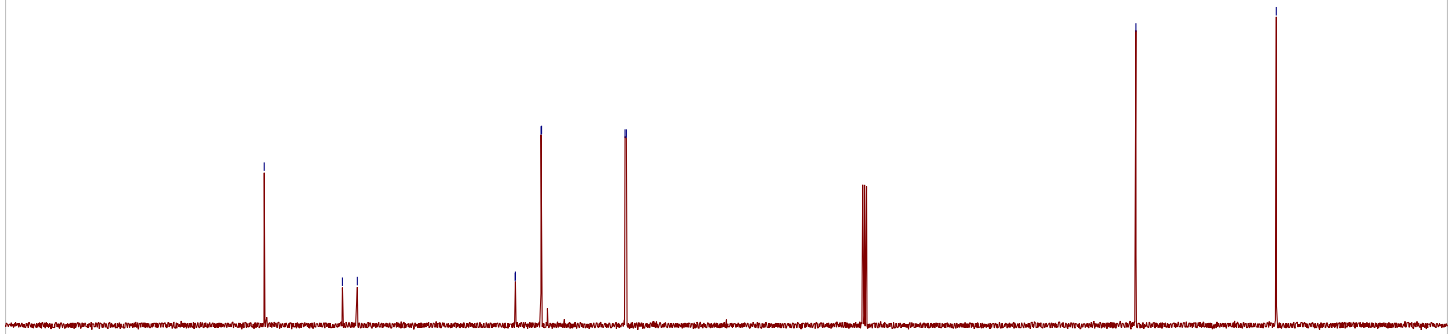

$\begin{array}{lllllllllllllllllllllll}210 & 200 & 190 & 180 & 170 & 160 & 150 & 140 & 130 & 120 & 110 & 100 & 90 & 80 & 70 & 60 & 50 & 40 & 30 & 20 & 10 & 0 & -10\end{array}$ 


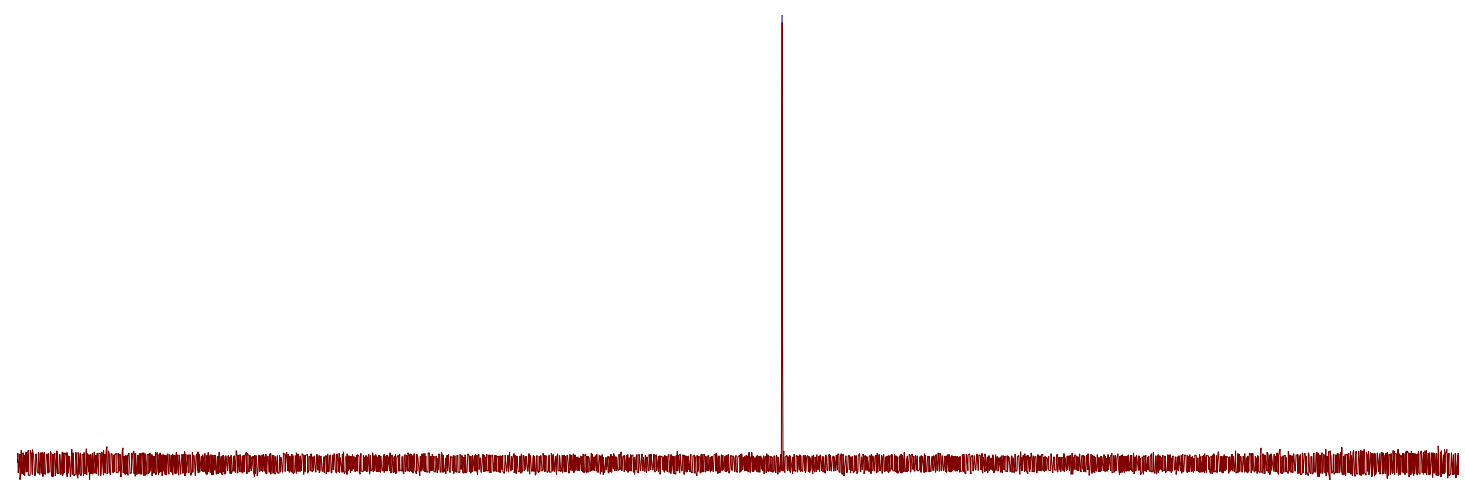

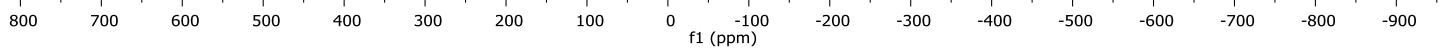<smiles>CCC(=O)N(C(=O)CC)c1ccc(F)cc1</smiles>

${ }^{19} \mathrm{~F}$ NMR $\left(56 \mathrm{MHz}, \mathrm{CDCl}_{3}\right)$ 
csk-2-101-3.10.fid

PROTON CDCI3 \{D:INMR400IDNL0604\} nmr-new 49

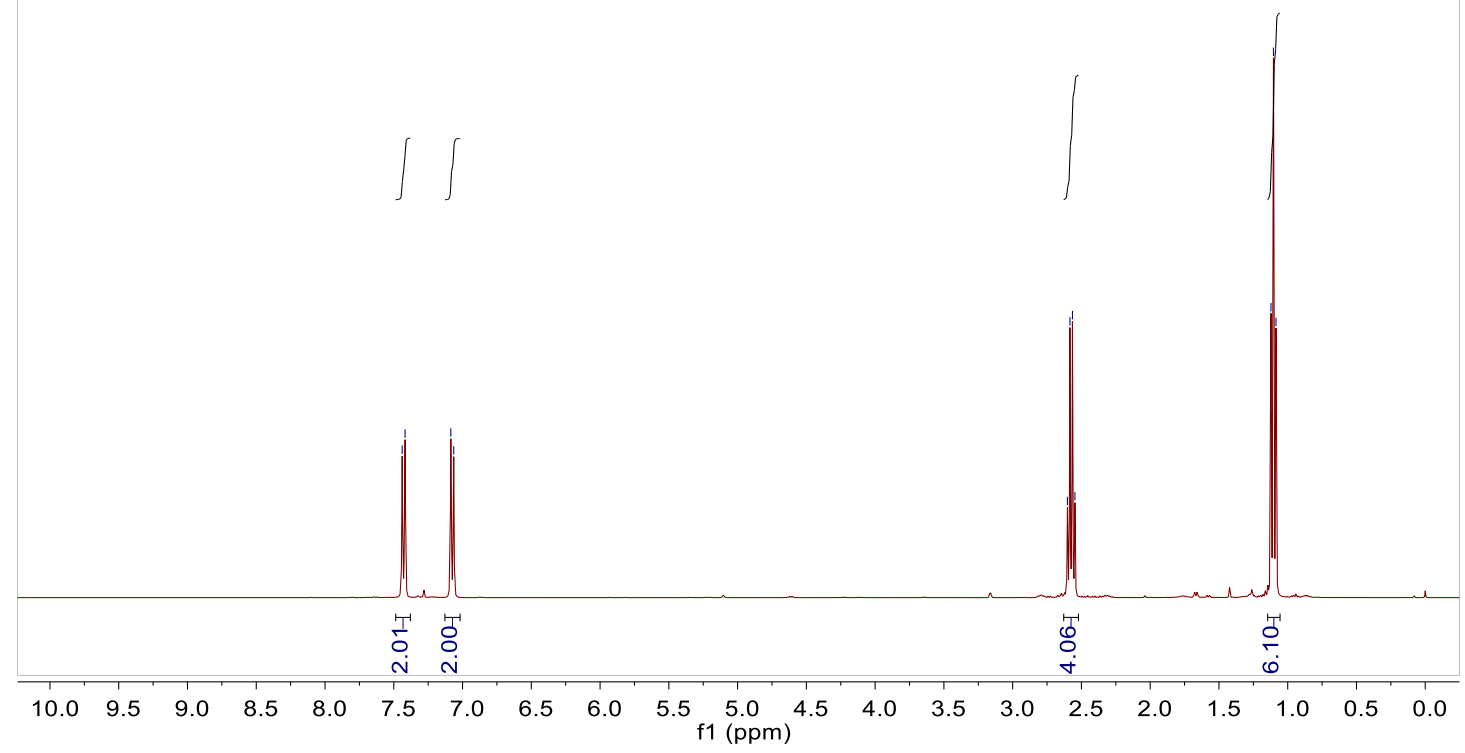

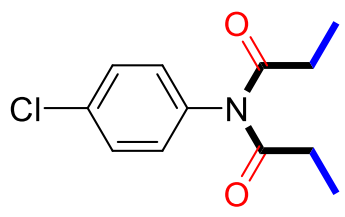

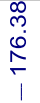

두ㅇㅛㅛ윰

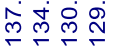

i
13

${ }^{1} \mathrm{H}$ NMR $\left(400 \mathrm{MHz}, \mathrm{CDCl}_{3}\right)$

${ }^{13} \mathrm{C} \mathrm{NMR}\left(100 \mathrm{MHz}, \mathrm{CDCl}_{3}\right)$

csk-2-101-3.11.fid

C13CPD CDCI3 \{D:INMR400IDNL0604\} nmr-new 49

\begin{tabular}{cc}
$\infty$ & \\
& \multicolumn{2}{c}{} \\
1 & $\infty$ \\
1 & 1
\end{tabular}

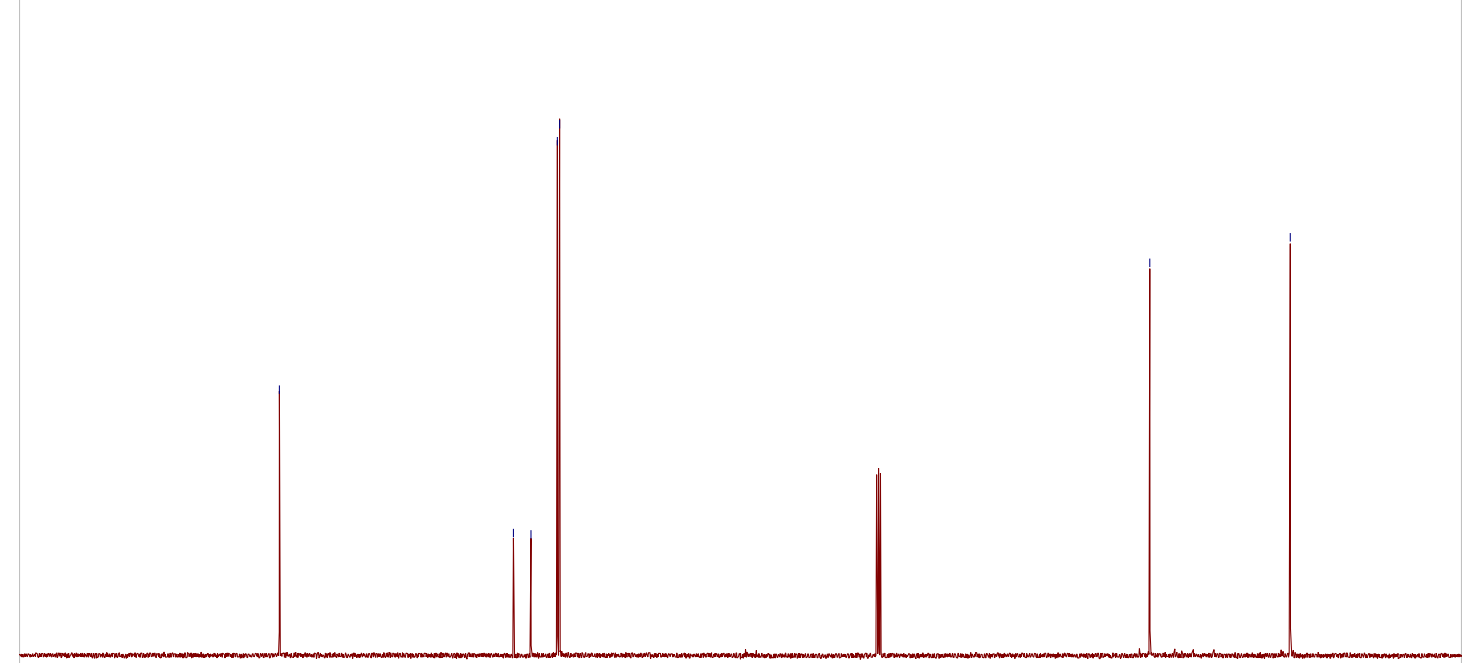

$\begin{array}{lllllllllllllllllllllll}210 & 200 & 190 & 180 & 170 & 160 & 150 & 140 & 130 & 120 & 110 & 100 & 90 & 80 & 70 & 60 & 50 & 40 & 30 & 20 & 10 & 0 & -10\end{array}$ 
csk-2-101-2.20.fid

PROTON CDCI3 \{D:INMR400ldnl0604\} nmr-new 1
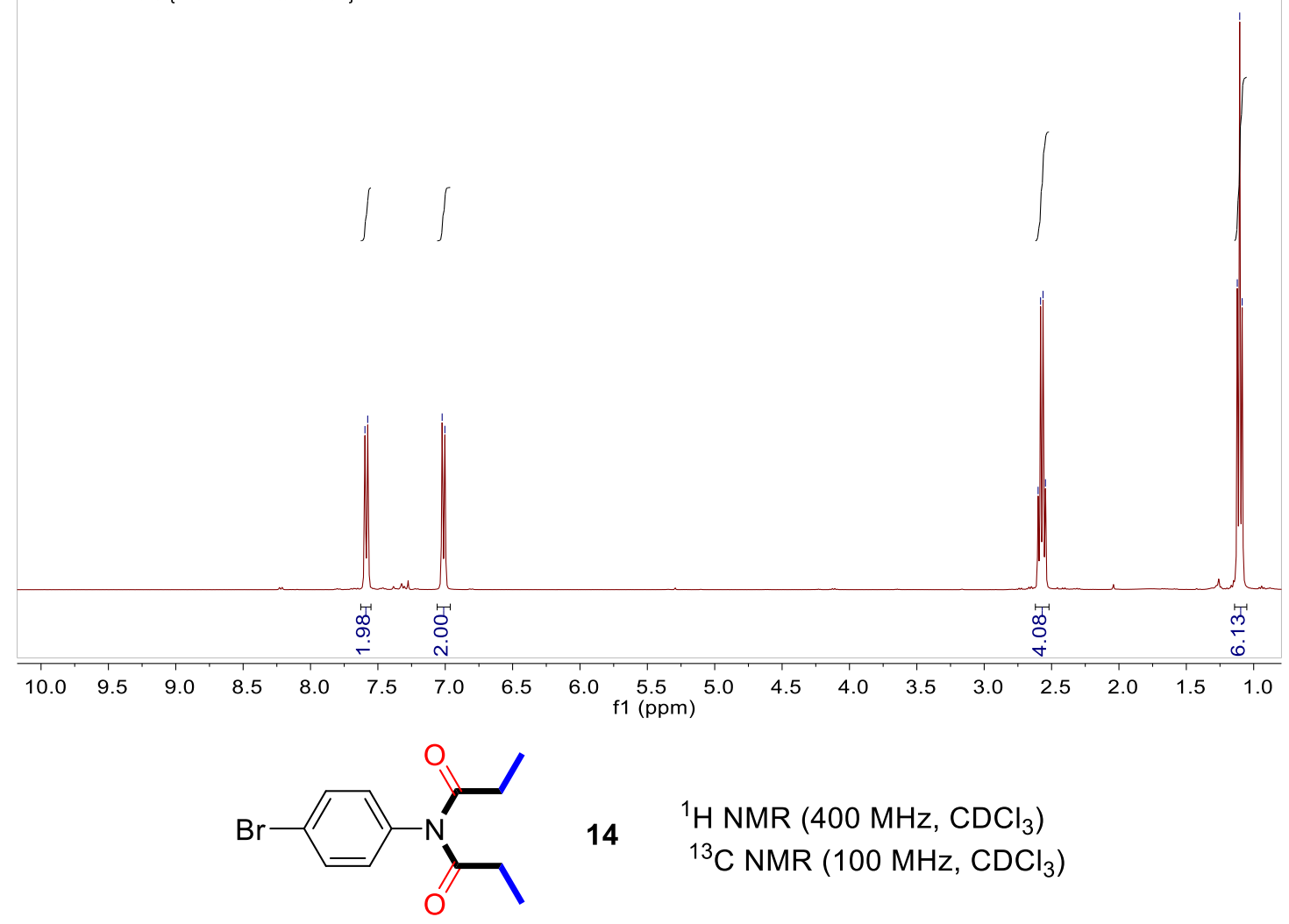

$14{ }^{1} \mathrm{H}$ NMR $\left(400 \mathrm{MHz}, \mathrm{CDCl}_{3}\right)$

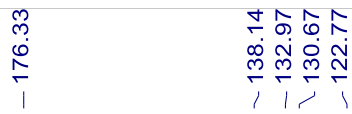

$\begin{array}{cc}\text { N } & 5 \\ \text { ले } & \text { o } \\ 1 & 1\end{array}$

csk-2-101-2.21.fid

C13CPD CDCI3 \{D:INMR400ldnl0604\} nmr-new 1

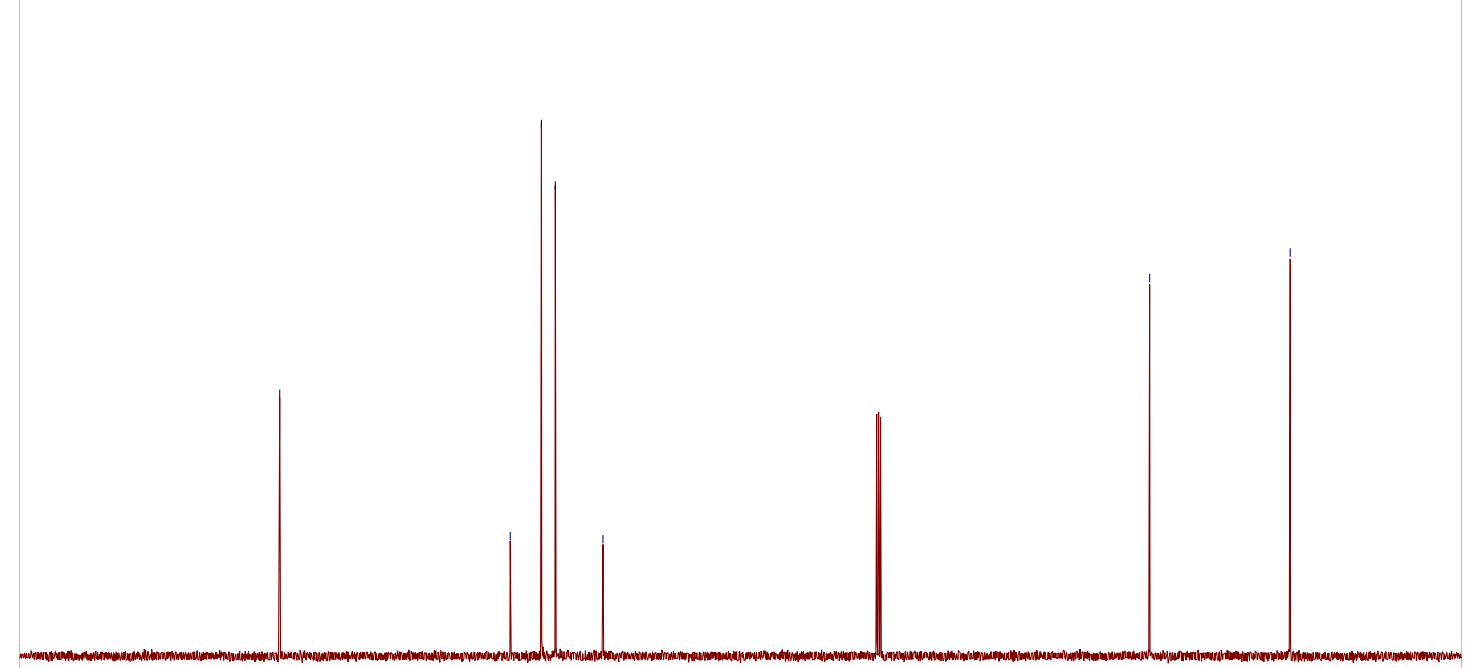

$\begin{array}{lllllllllllllllllllllll}210 & 200 & 190 & 180 & 170 & 160 & 150 & 140 & 130 & 120 & 110 & 100 & 90 & 80 & 70 & 60 & 50 & 40 & 30 & 20 & 10 & 0 & -10\end{array}$ 


\begin{tabular}{|c|c|}
\hline 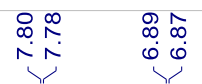 & نئ \\
\hline
\end{tabular}

csk-3-5-10.10.fid

PROTON CDCI3 \{D:INMR400ldnl0604\} nmr-new 2
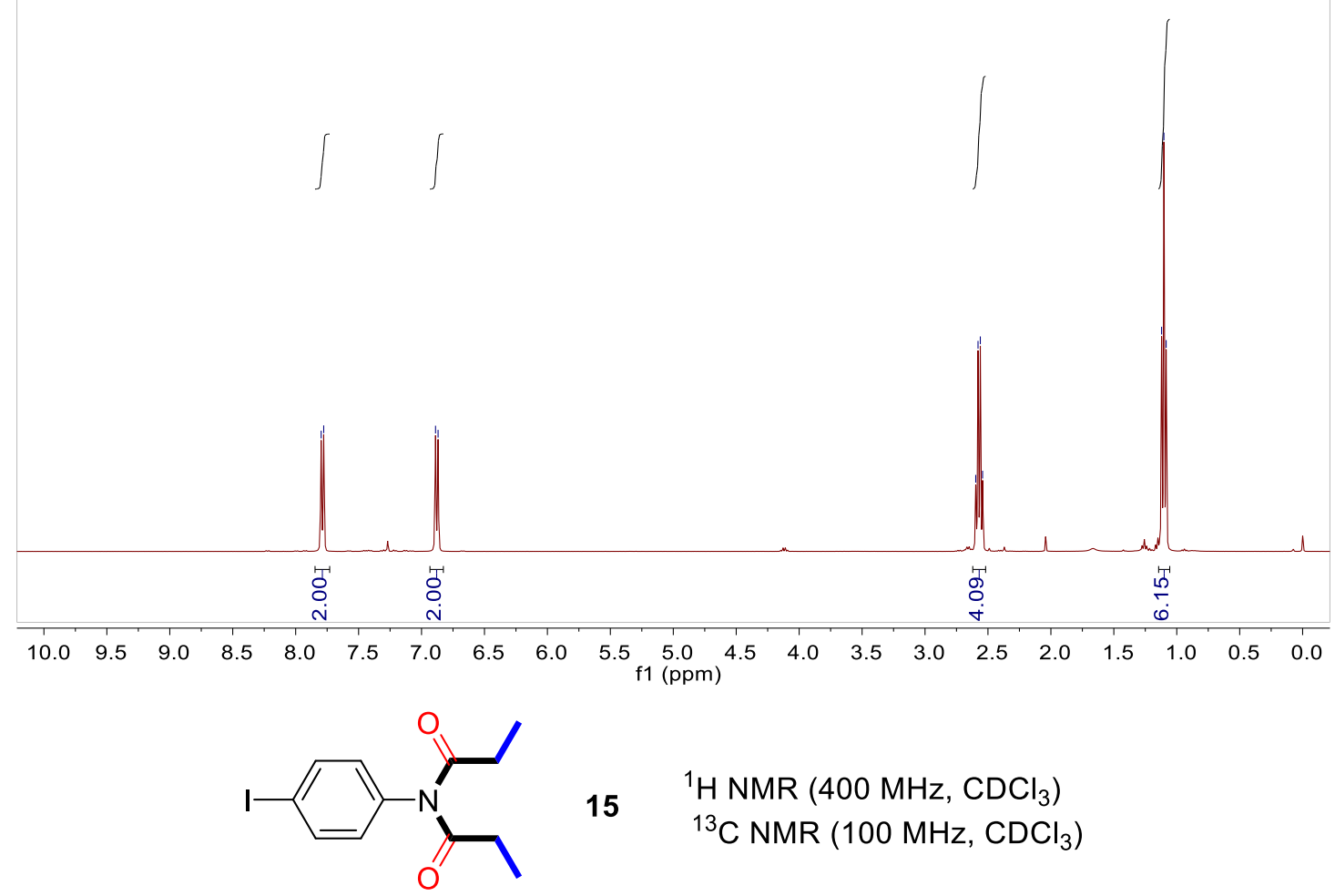

$15{ }^{1} \mathrm{H} \mathrm{NMR}\left(400 \mathrm{MHz}, \mathrm{CDCl}_{3}\right)$

${ }^{13} \mathrm{C}$ NMR $\left(100 \mathrm{MHz}, \mathrm{CDCl}_{3}\right)$

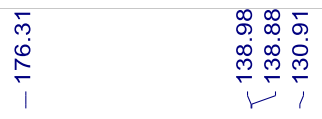

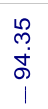

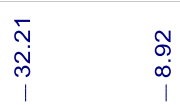

csk-3-5-10.11.fid

C13CPD CDCI3 \{D:INMR400ldnl0604\} nmr-new 16

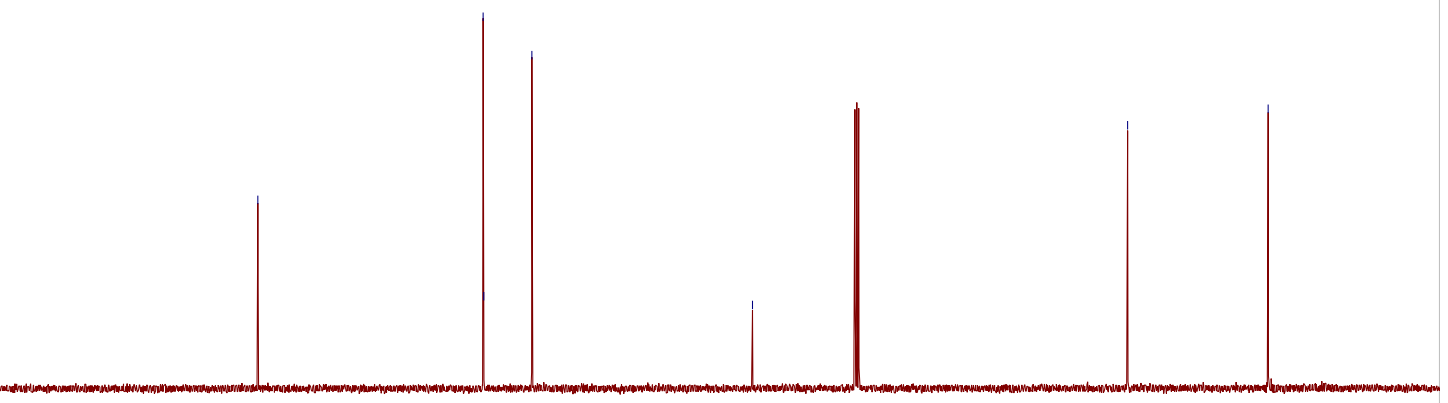

$\begin{array}{llllllllllllllllllllllll}210 & 200 & 190 & 180 & 170 & 160 & 150 & 140 & 130 & 120 & 110 & 100 & 90 & 80 & 70 & 60 & 50 & 40 & 30 & 20 & 10 & 0 & -10\end{array}$ 
csk-3-5-1.10.fid

PROTON CDCI3 \{D:INMR400ldnl0604\} nmr-new 3
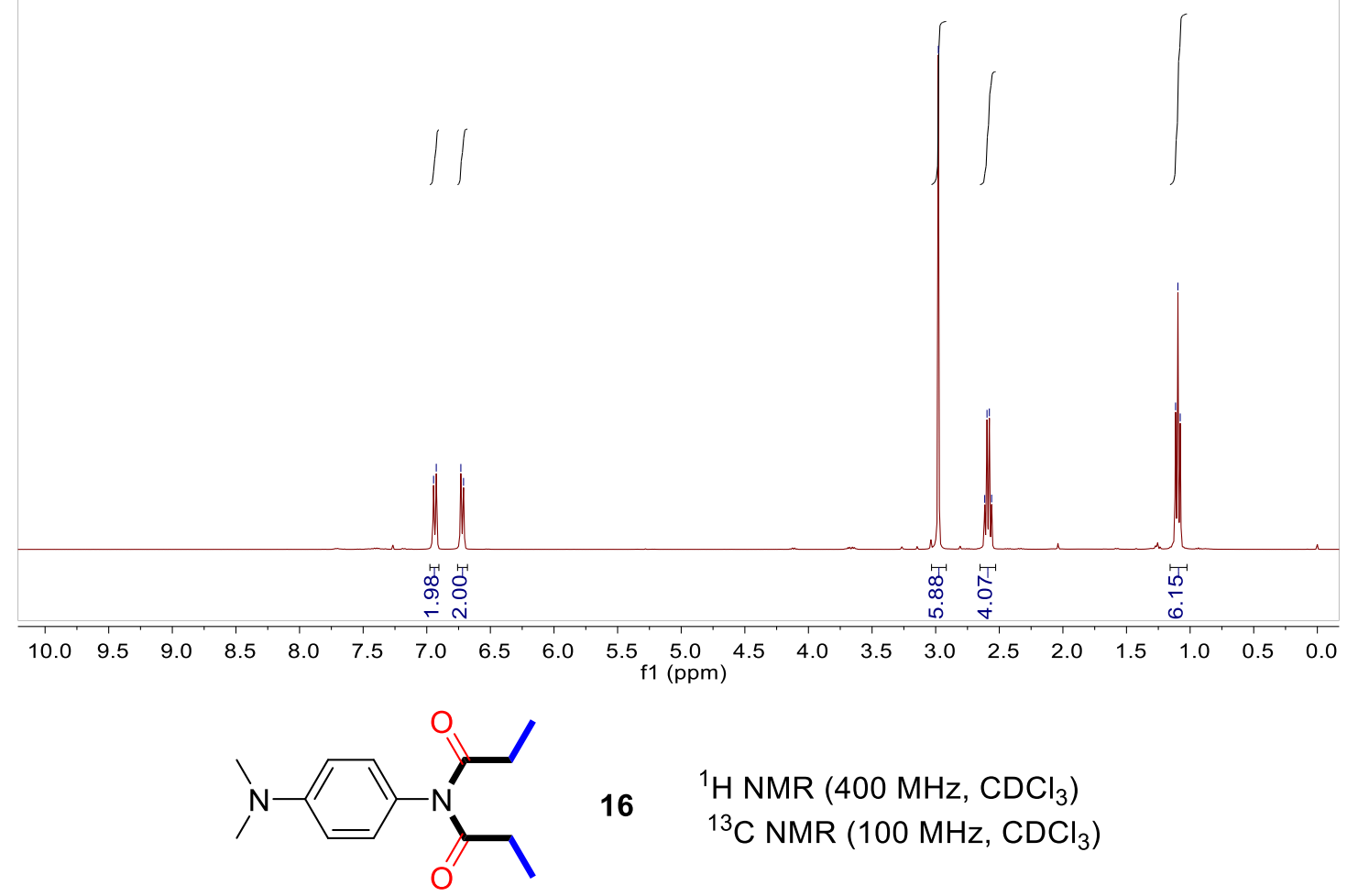

$16{ }^{1} \mathrm{H}$ NMR $\left(400 \mathrm{MHz}, \mathrm{CDCl}_{3}\right)$

${ }^{13} \mathrm{C}$ NMR (100 MHz, $\mathrm{CDCl}_{3}$ )

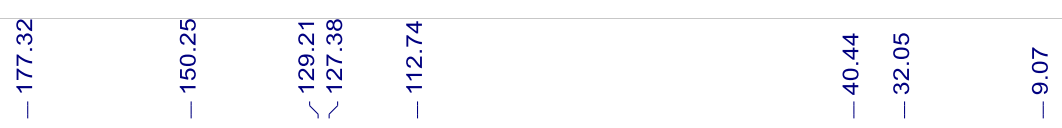

csk-3-5-1.11.fid

C13CPD CDCI3 \{D:INMR400ldn10604\} nmr-new 3

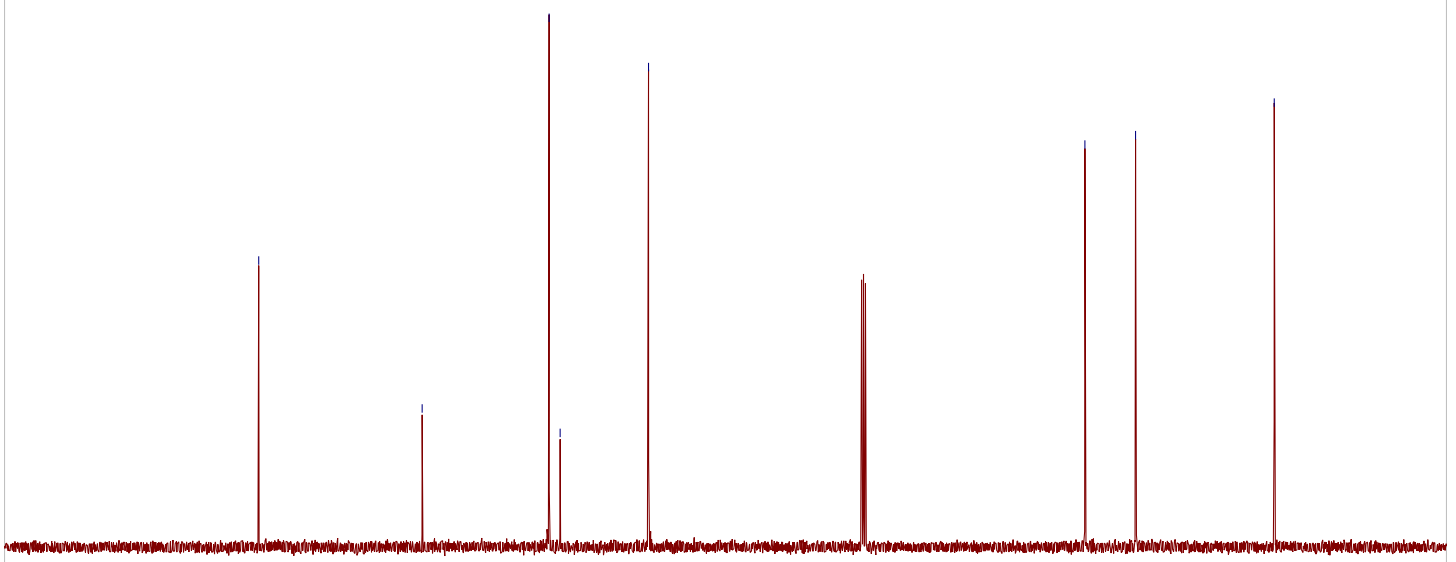

$\begin{array}{lllllllllllllllllllllll}210 & 200 & 190 & 180 & 170 & 160 & 150 & 140 & 130 & 120 & 110 & 100 & 90 & 80 & 70 & 60 & 50 & 40 & 30 & 20 & 10 & 0 & -10\end{array}$ f1 (ppm) 
csk-3-5-18.10.fid

PROTON CDCI3 \{D:INMR400ldn10604\} nmr-new 34
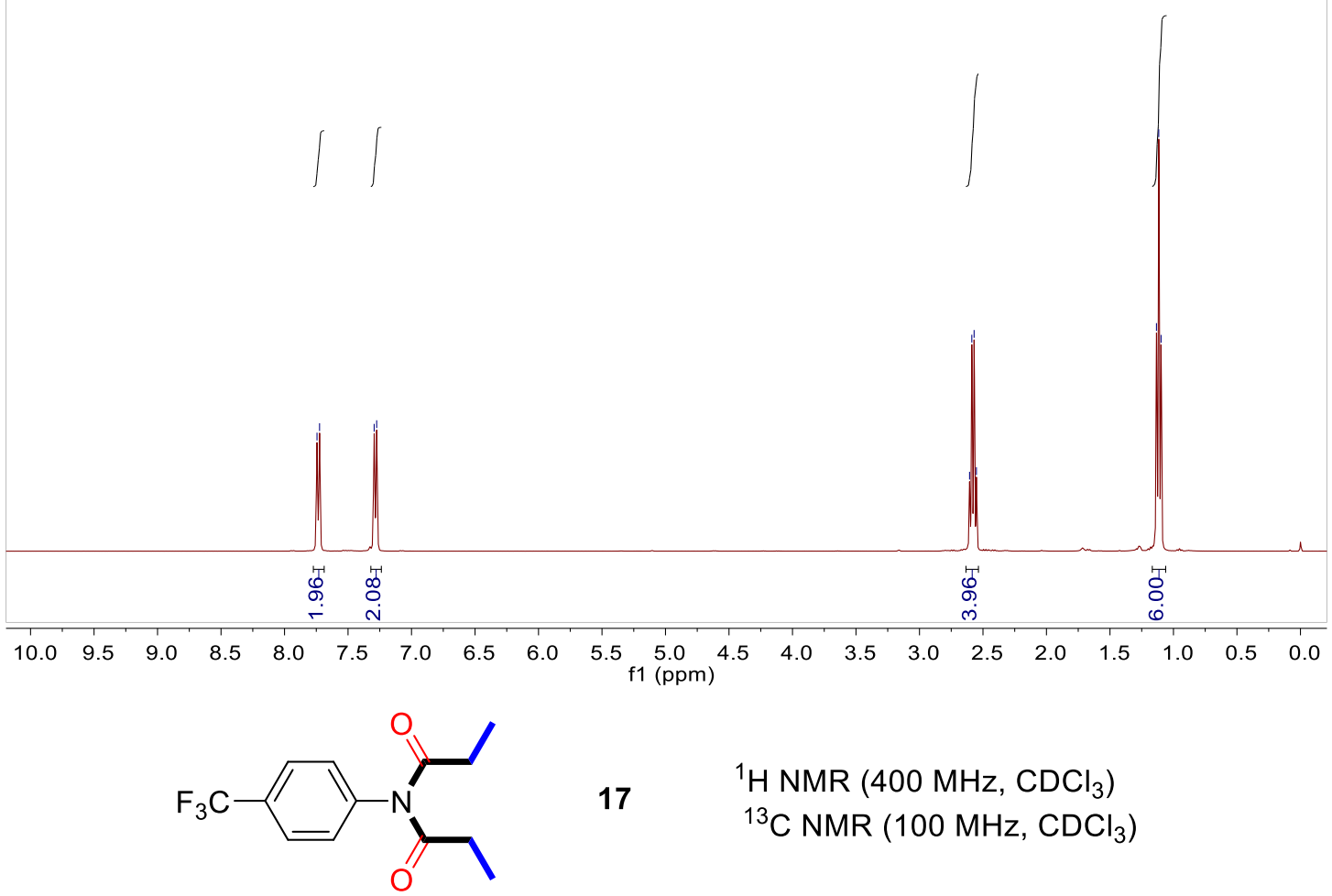

17

${ }^{1} \mathrm{H}$ NMR $\left(400 \mathrm{MHz}, \mathrm{CDCl}_{3}\right)$

${ }^{13} \mathrm{C}$ NMR $\left(100 \mathrm{MHz}, \mathrm{CDCl}_{3}\right)$

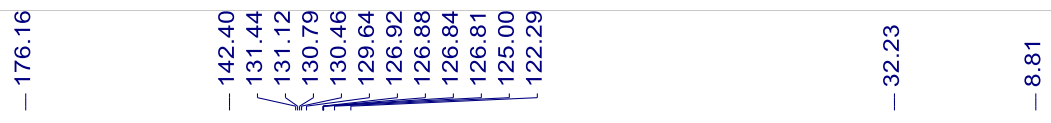

csk-3-5-18.11.fid

C13CPD CDCI3 \{D:INMR400ldn10604\} nmr-new 34

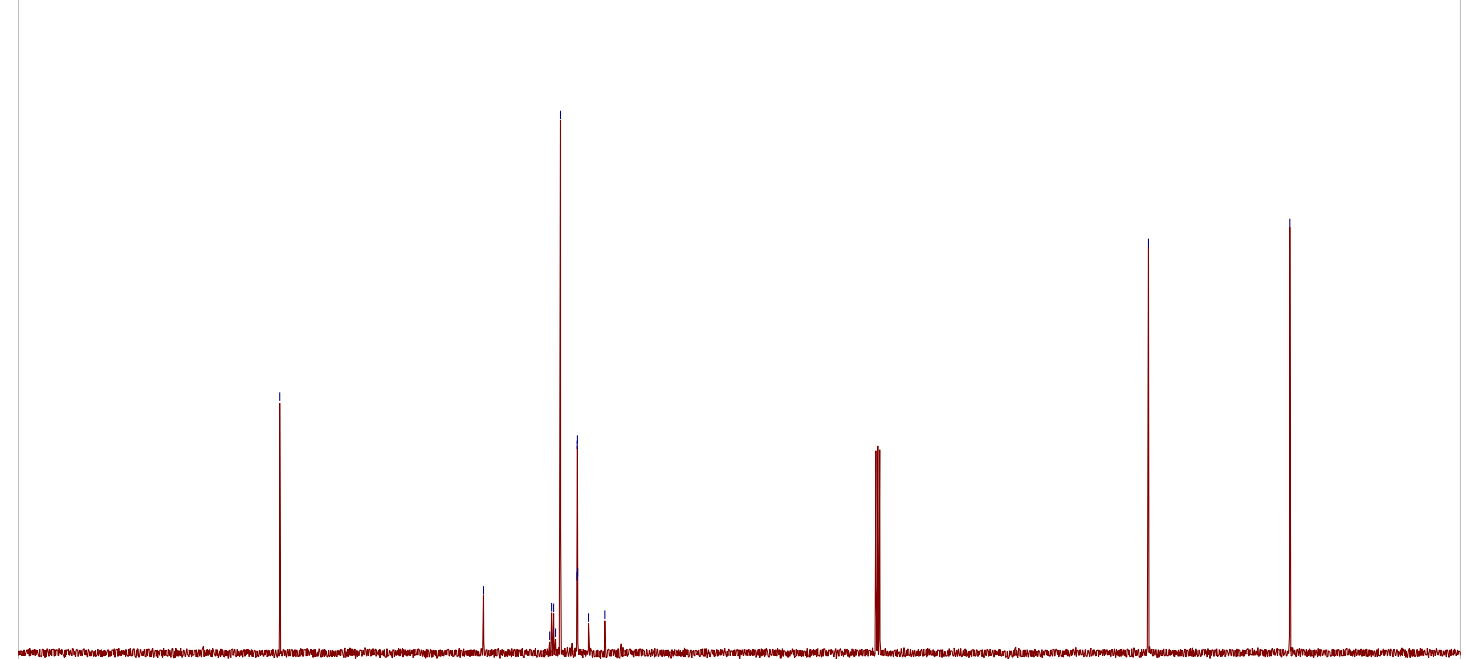

$\begin{array}{lllllllllllllllllllllll}210 & 200 & 190 & 180 & 170 & 160 & 150 & 140 & 130 & 120 & 110 & 100 & 90 & 80 & 70 & 60 & 50 & 40 & 30 & 20 & 10 & 0 & -10\end{array}$ 


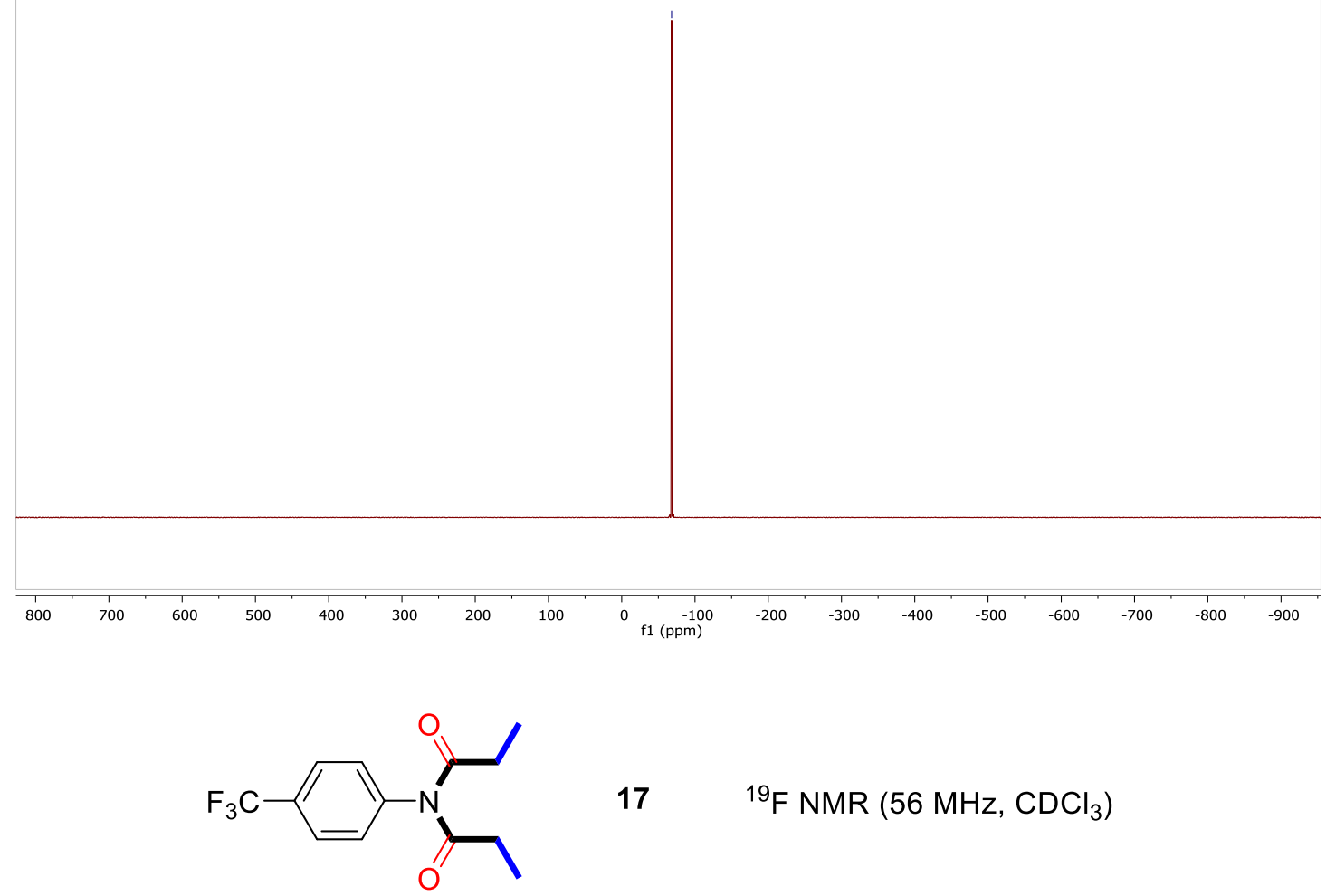



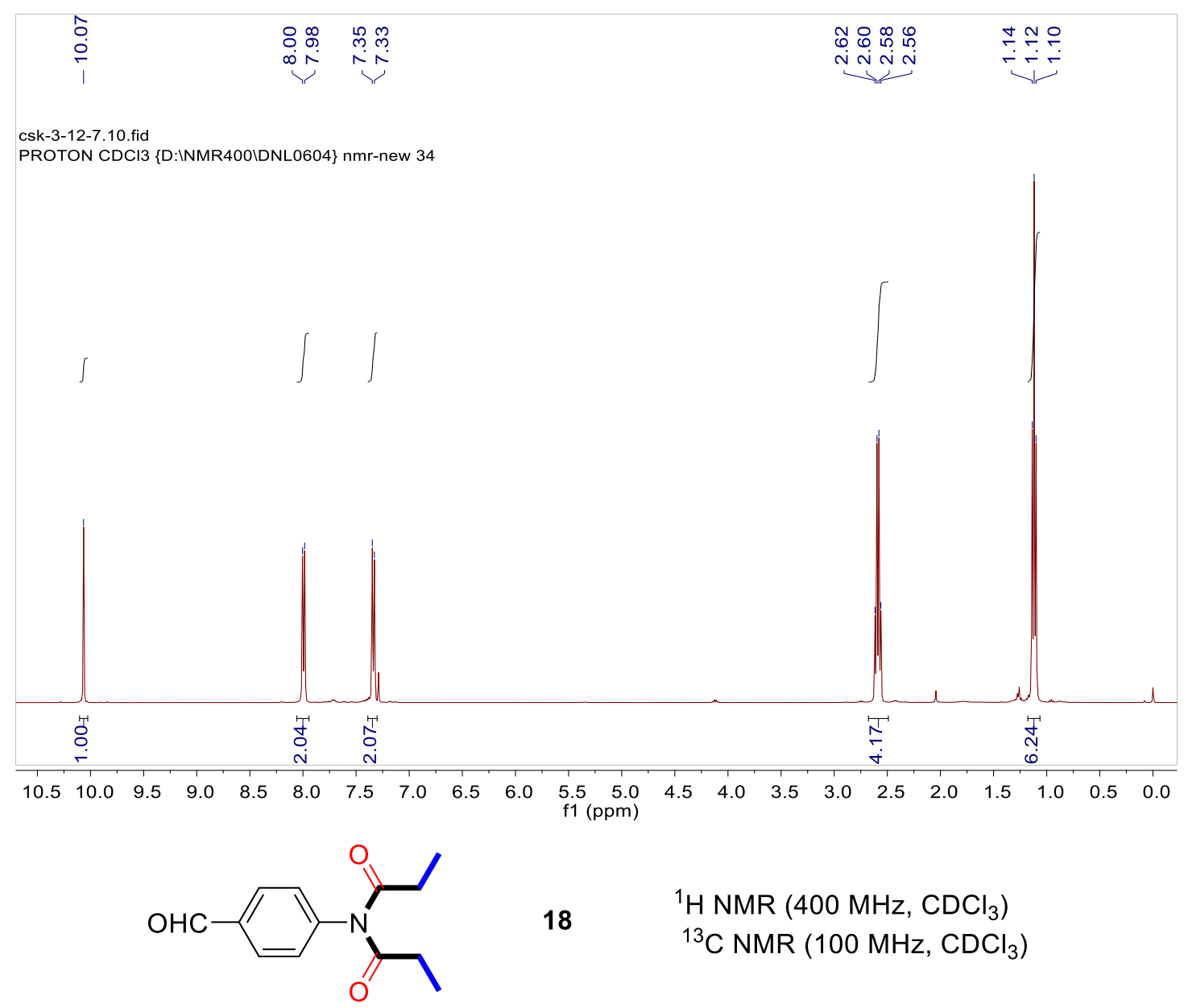

${ }^{13} \mathrm{C}$ NMR $\left(100 \mathrm{MHz}, \mathrm{CDCl}_{3}\right)$

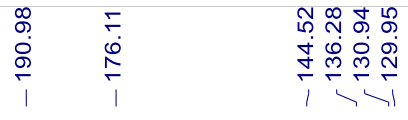

csk-3-12-7.11.fid

C13CPD CDCI3 \{D:INMR400IDNL0604\} nmr-new 34

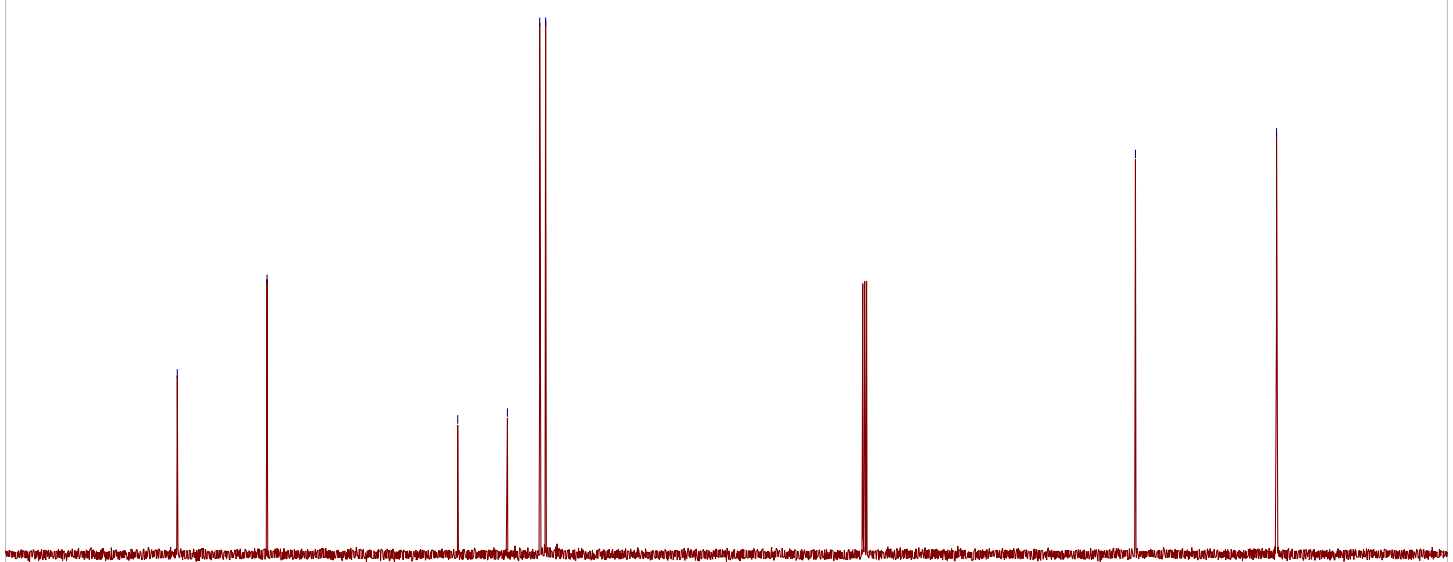

$\begin{array}{lllllllllllllllllllllll}210 & 200 & 190 & 180 & 170 & 160 & 150 & 140 & 130 & 120 & 110 & 100 & 90 & 80 & 70 & 60 & 50 & 40 & 30 & 20 & 10 & 0 & -10\end{array}$ 


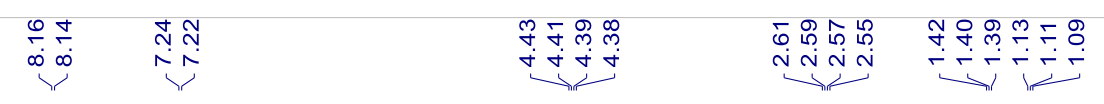

csk-3-12-9.10.fid

PROTON CDCI3 \{D:INMR400IDNL0604\} nmr-new 36
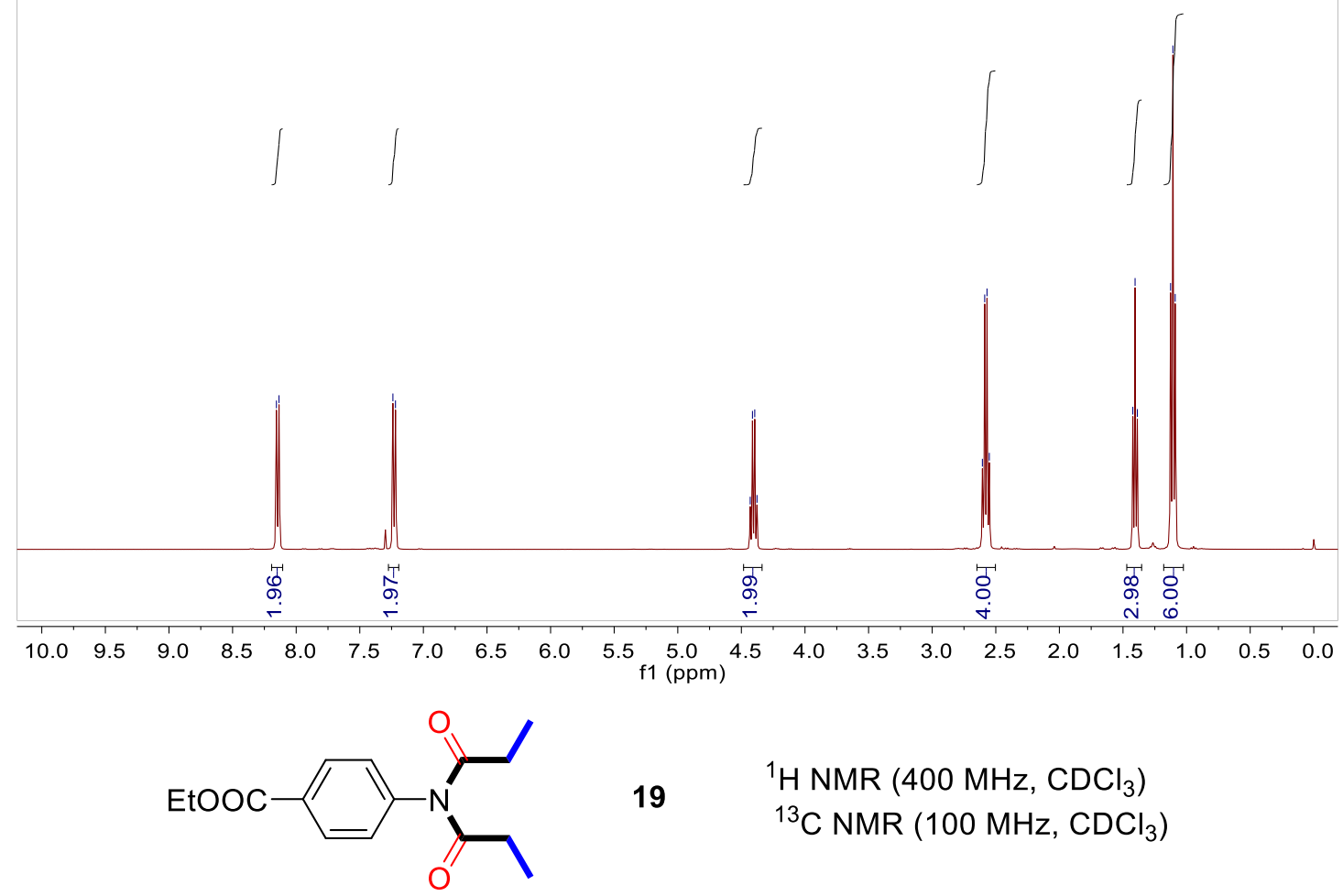

19

${ }^{1} \mathrm{H} \mathrm{NMR}\left(400 \mathrm{MHz}, \mathrm{CDCl}_{3}\right)$

${ }^{13} \mathrm{C} \mathrm{NMR}\left(100 \mathrm{MHz}, \mathrm{CDCl}_{3}\right)$

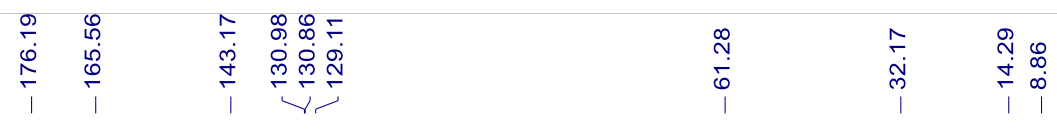

csk-3-12-9.11.fid

C13CPD CDCI3 \{D:INMR400IDNL0604\} nmr-new 36

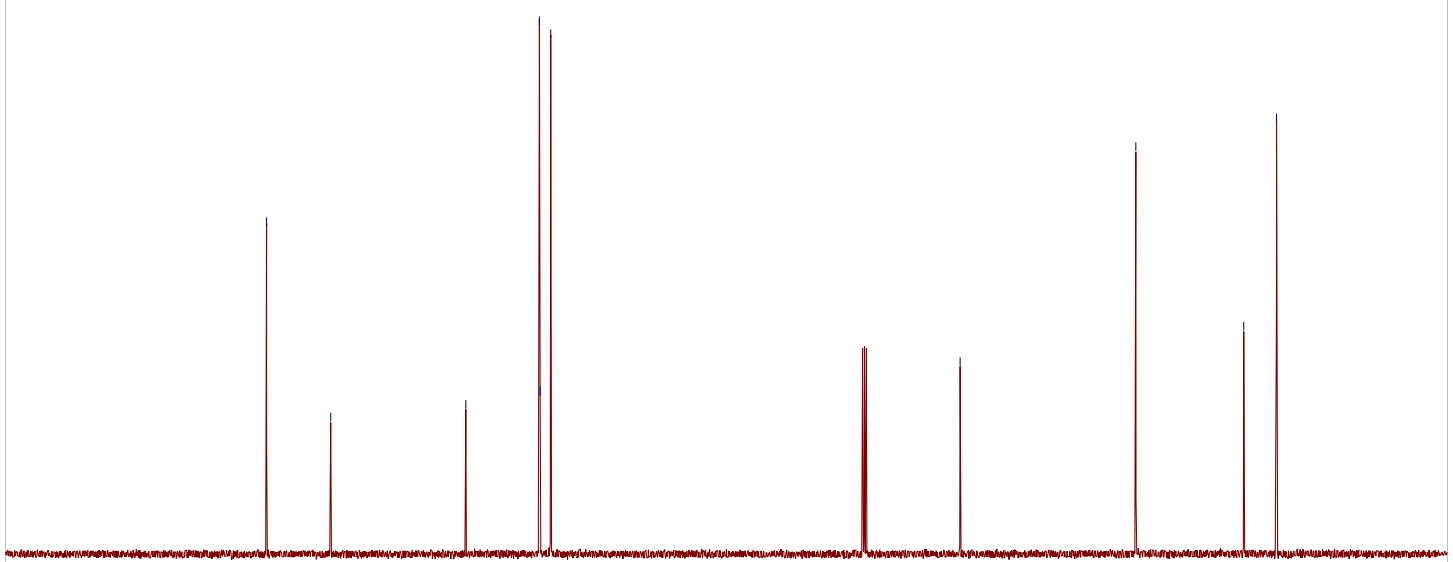

$\begin{array}{lllllllllllllllllllllll}210 & 200 & 190 & 180 & 170 & 160 & 150 & 140 & 130 & 120 & 110 & 100 & 90 & 80 & 70 & 60 & 50 & 40 & 30 & 20 & 10 & 0 & -10\end{array}$ 
csk-3-19-10.10.fid

PROTON CDCI3 \{D:INMR400IDNL0604\} nmr-new 12
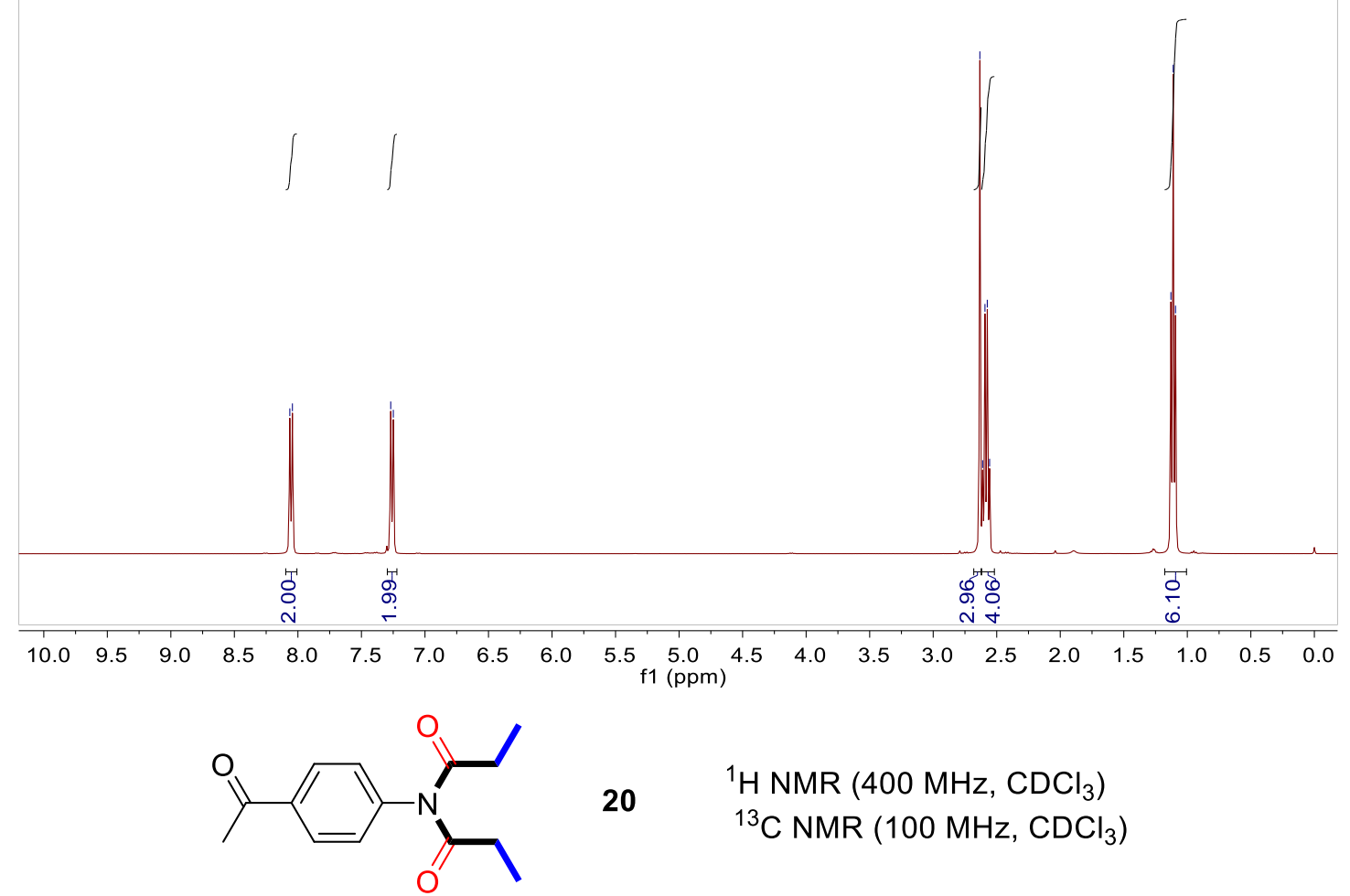

20

${ }^{1} \mathrm{H}$ NMR $\left(400 \mathrm{MHz}, \mathrm{CDCl}_{3}\right)$

${ }^{13} \mathrm{C}$ NMR $\left(100 \mathrm{MHz}, \mathrm{CDCl}_{3}\right)$

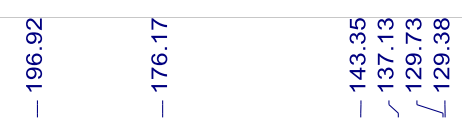

$\stackrel{\infty}{\circ} \mathbb{0}$

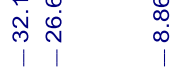

csk-3-19-10.11.fid

C13CPD CDCI3 \{D:INMR400IDNL0604\} nmr-new 12

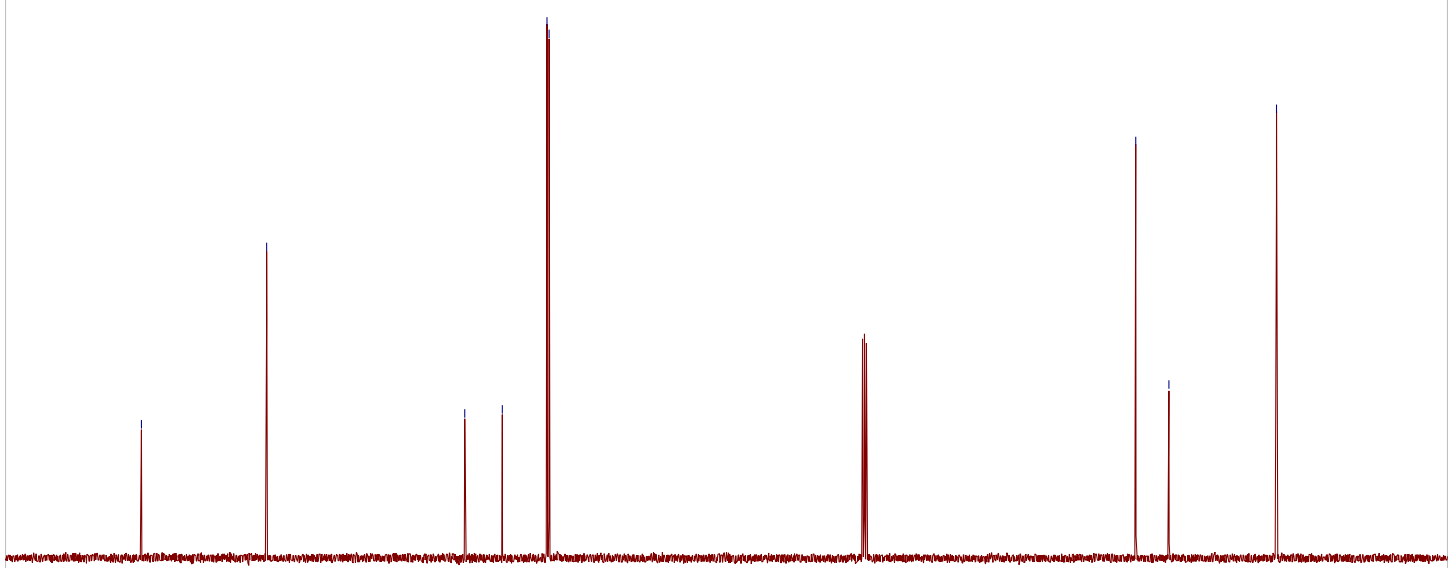

$\begin{array}{lllllllllllllllllllllllllll}210 & 200 & 190 & 180 & 170 & 160 & 150 & 140 & 130 & 120 & 110 & 100 & 90 & 80 & 70 & 60 & 50 & 40 & 30 & 20 & 10 & 0 & -10\end{array}$ 
csk-2-101-5.10.fid
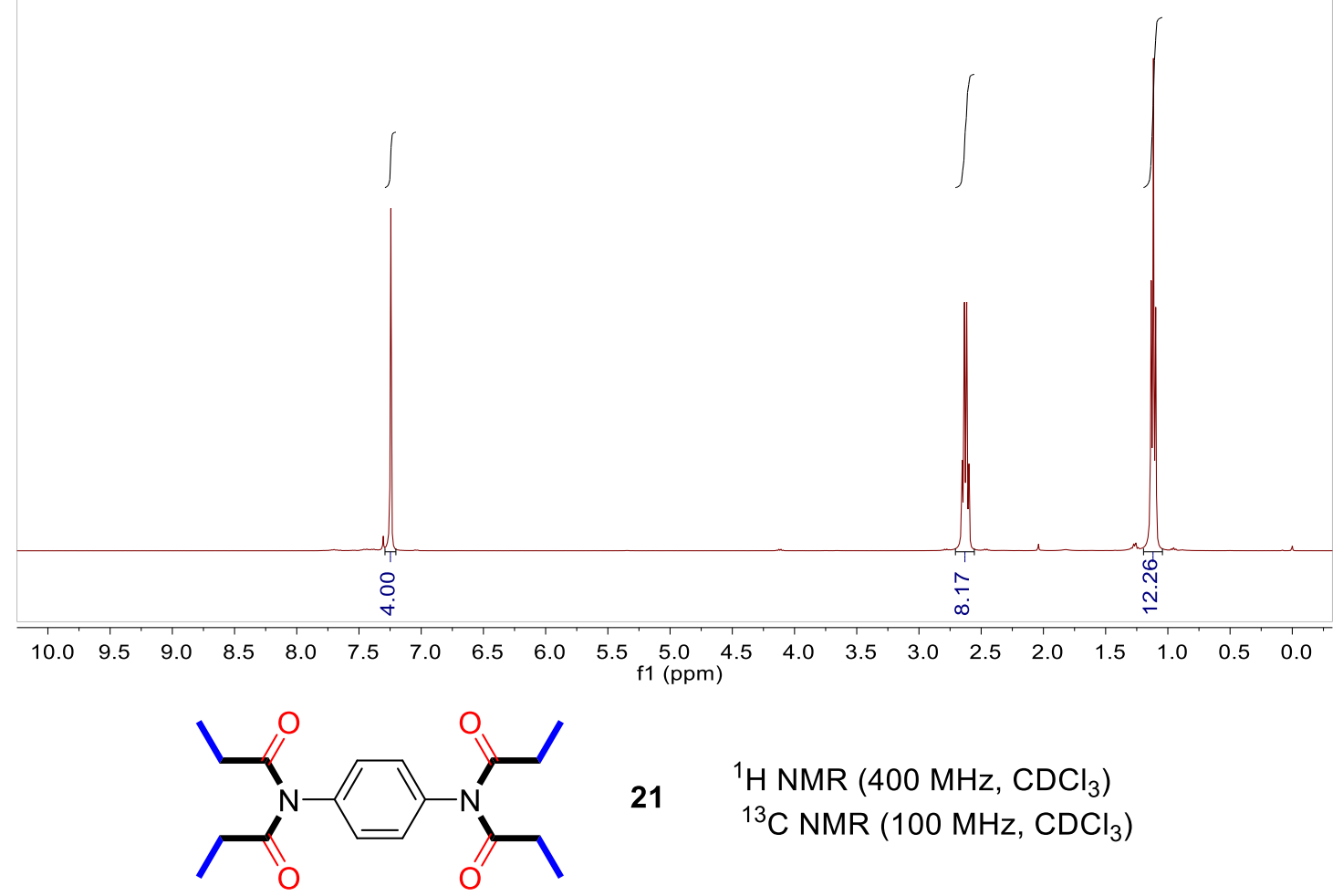

21

${ }^{1} \mathrm{H}$ NMR $\left(400 \mathrm{MHz}, \mathrm{CDCl}_{3}\right)$

${ }^{13} \mathrm{C} \mathrm{NMR}\left(100 \mathrm{MHz}, \mathrm{CDCl}_{3}\right)$
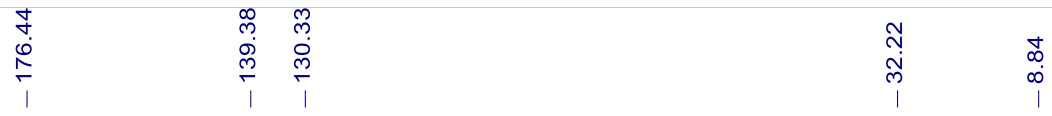

csk-2-101-5.11.fid

C13CPD CDCI3 \{D:INMR400ldnl0604\} nmr-new 20

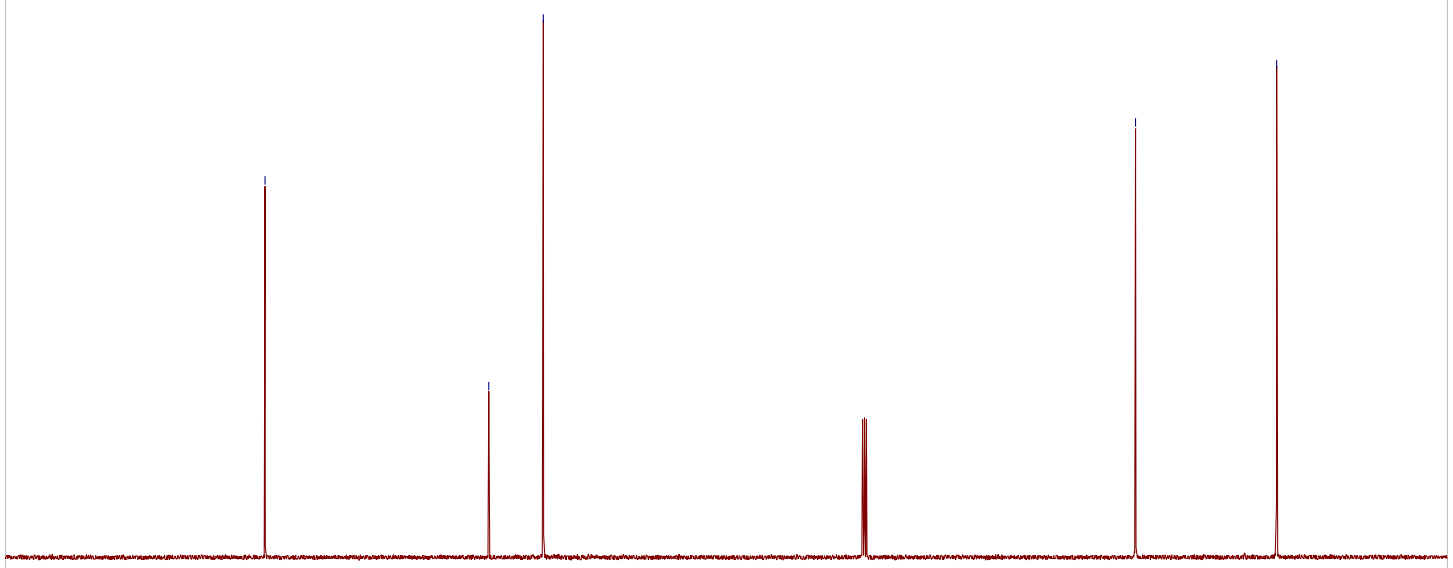

$\begin{array}{llllllllllllllllllllllll}210 & 200 & 190 & 180 & 170 & 160 & 150 & 140 & 130 & 120 & 110 & 100 & 90 & 80 & 70 & 60 & 50 & 40 & 30 & 20 & 10 & 0 & -10\end{array}$ 
csk-3-5-3.10.fid

PROTON CDCI3 \{D:INMR400ldnl0604\} nmr-new 5
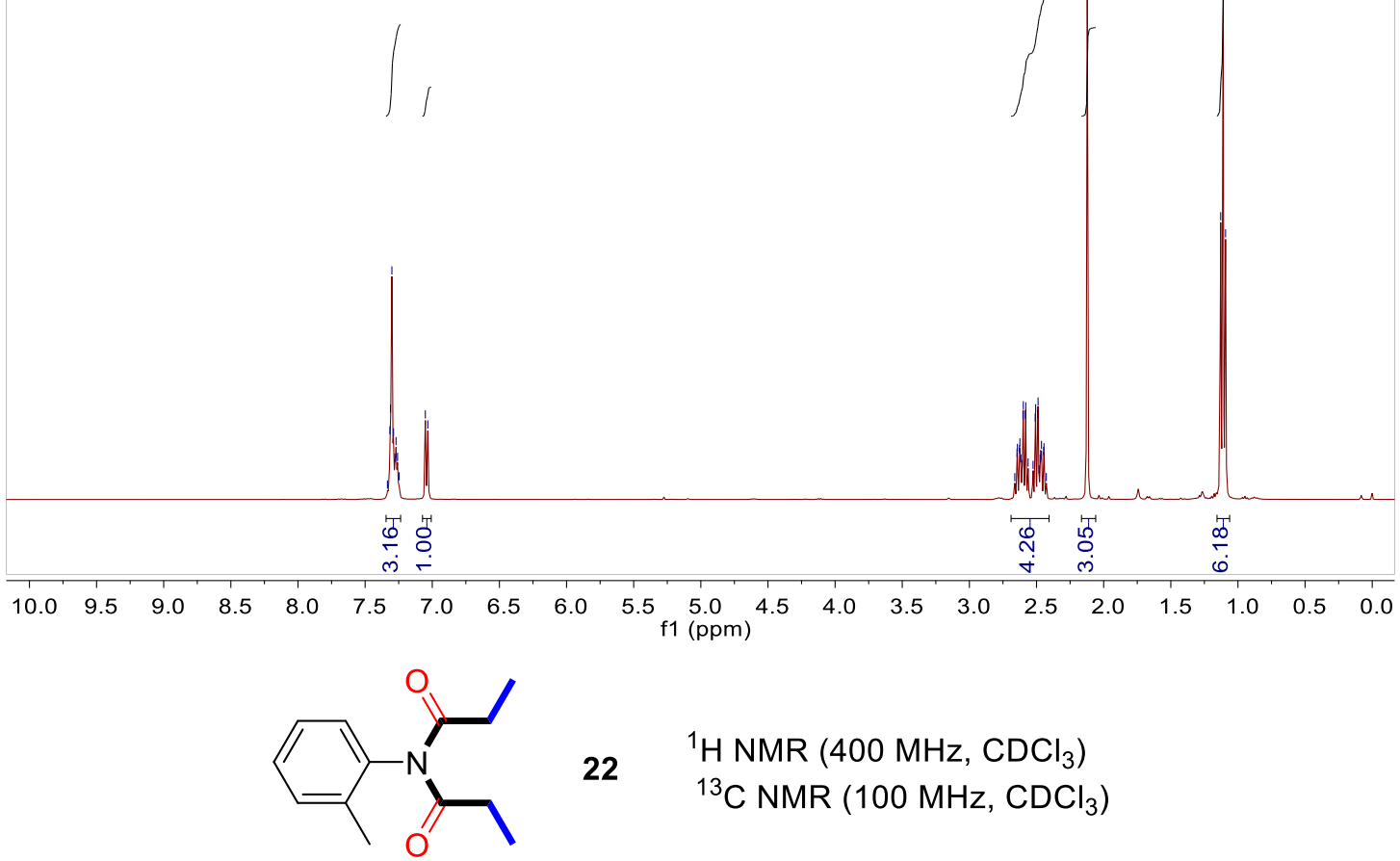

$22{ }^{1} \mathrm{H}$ NMR $\left(400 \mathrm{MHz}, \mathrm{CDCl}_{3}\right)$

${ }^{13} \mathrm{C} \mathrm{NMR}\left(100 \mathrm{MHz}, \mathrm{CDCl}_{3}\right)$

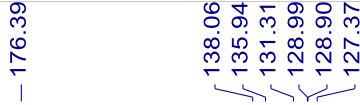

sk-3-5-3.11.fid

C13CPD CDCI3 \{D:INMR400Idnl0604\} nmr-new 5

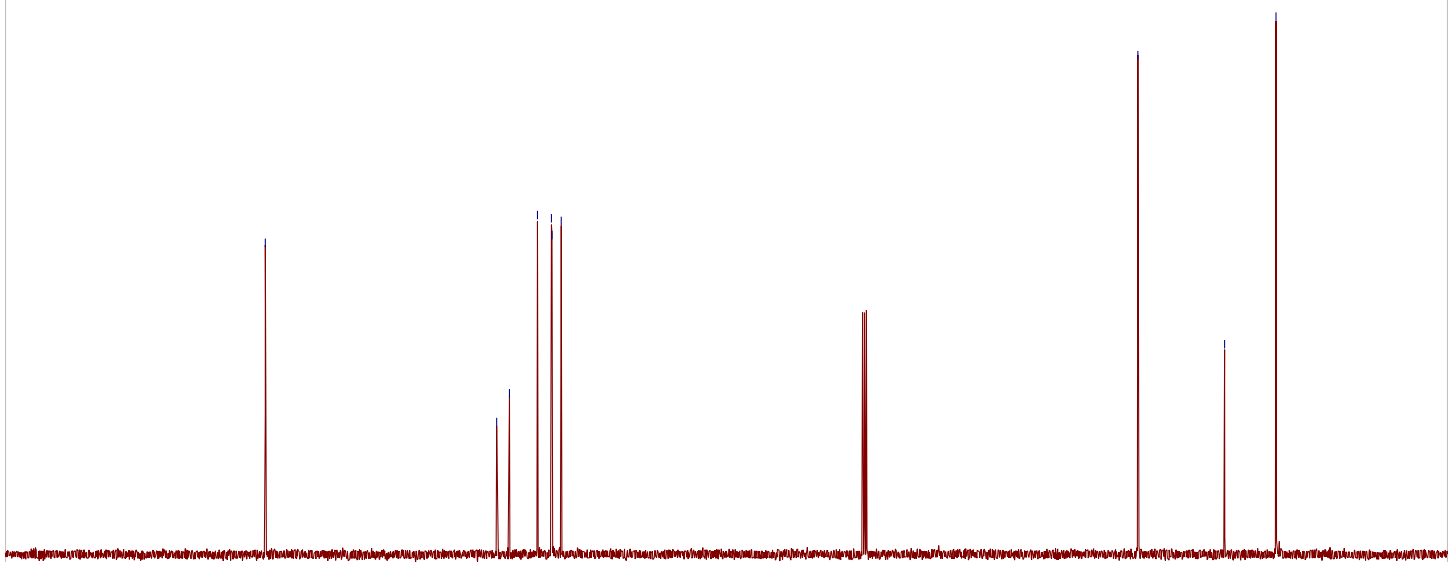

$\begin{array}{lllllllllllllllllllllll}210 & 200 & 190 & 180 & 170 & 160 & 150 & 140 & 130 & 120 & 110 & 100 & 90 & 80 & 70 & 60 & 50 & 40 & 30 & 20 & 10 & 0 & -10\end{array}$ 


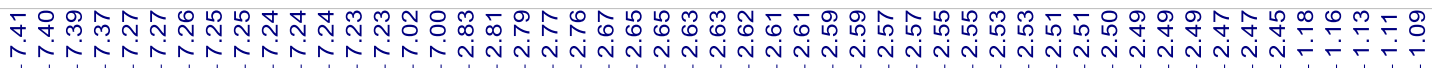

csk-3-5-7.10.fid

PROTON CDCI3 \{D:INMR400Idnl0604\} nmr-new 18

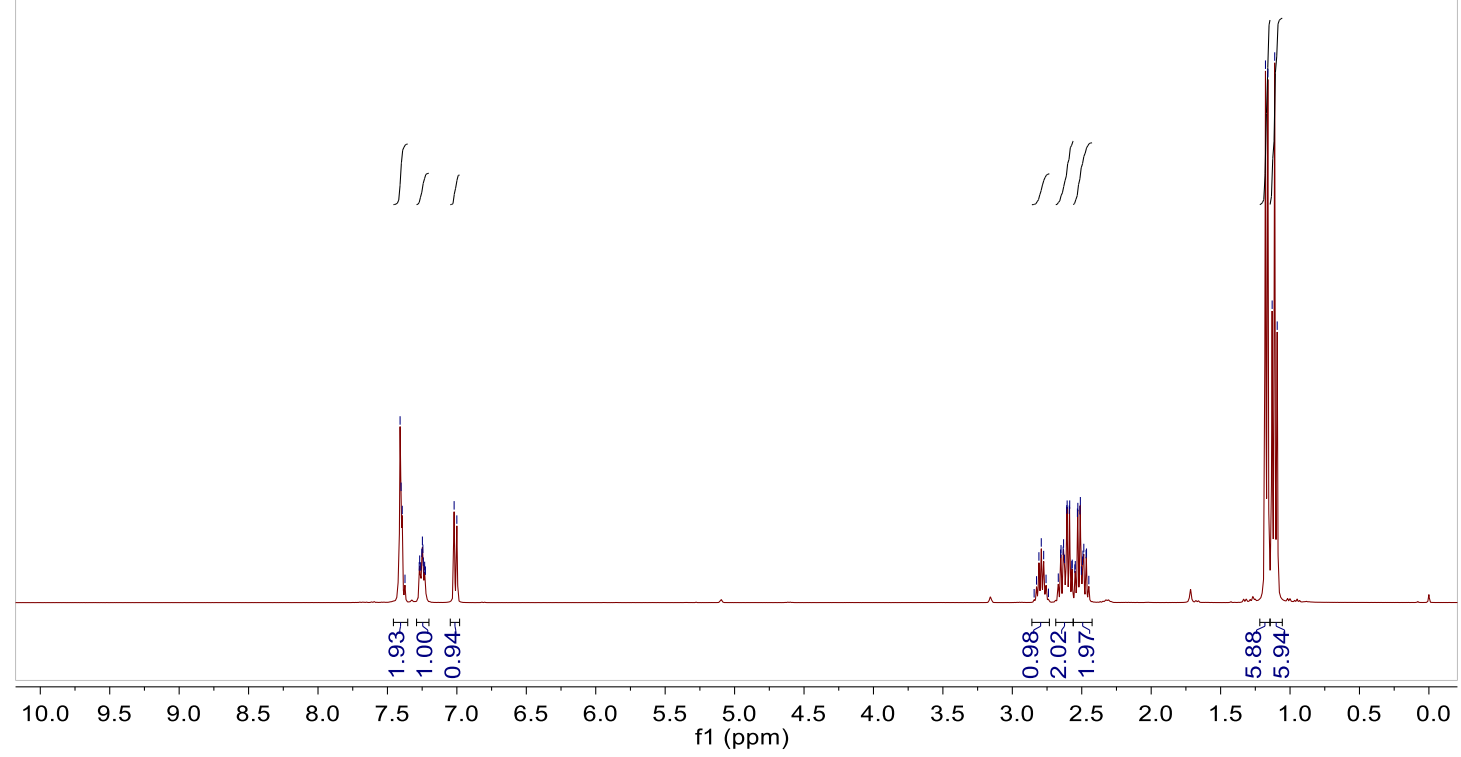

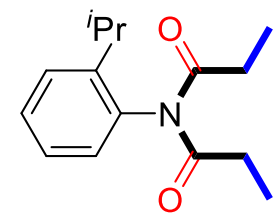

$\frac{i}{5}$

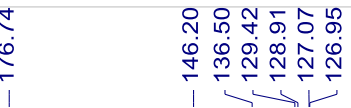

${ }^{1} \mathrm{H}$ NMR $\left(400 \mathrm{MHz}, \mathrm{CDCl}_{3}\right)$

${ }^{13} \mathrm{C}$ NMR $\left(100 \mathrm{MHz}, \mathrm{CDCl}_{3}\right)$

csk-3-5-7.11.fid

C13CPD CDCI3 \{D:INMR400ldnl0604\} nmr-new 18

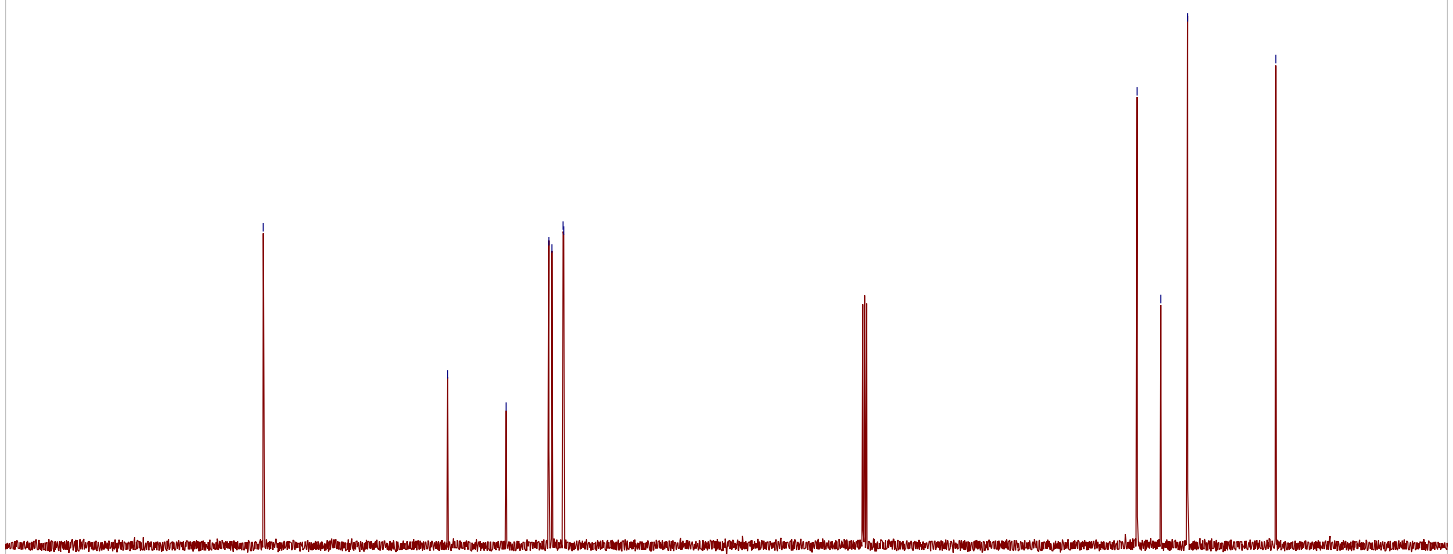

$\begin{array}{lllllllllllllllllllllll}210 & 200 & 190 & 180 & 170 & 160 & 150 & 140 & 130 & 120 & \begin{array}{c}110 \\ \mathrm{f} 1\end{array}(\mathrm{ppm})\end{array}$

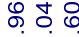

$\dot{m} \stackrel{\infty}{\sim} \stackrel{\dot{m}}{\sim}$

$\infty$
$\stackrel{\infty}{\infty}$
$\infty$

$\begin{array}{lllll}1 & 1 & 1\end{array}$ 
csk-3-5-14.10.fid

PROTON CDCI3 \{D:INMR400IDNL0604\} nmr-new 58
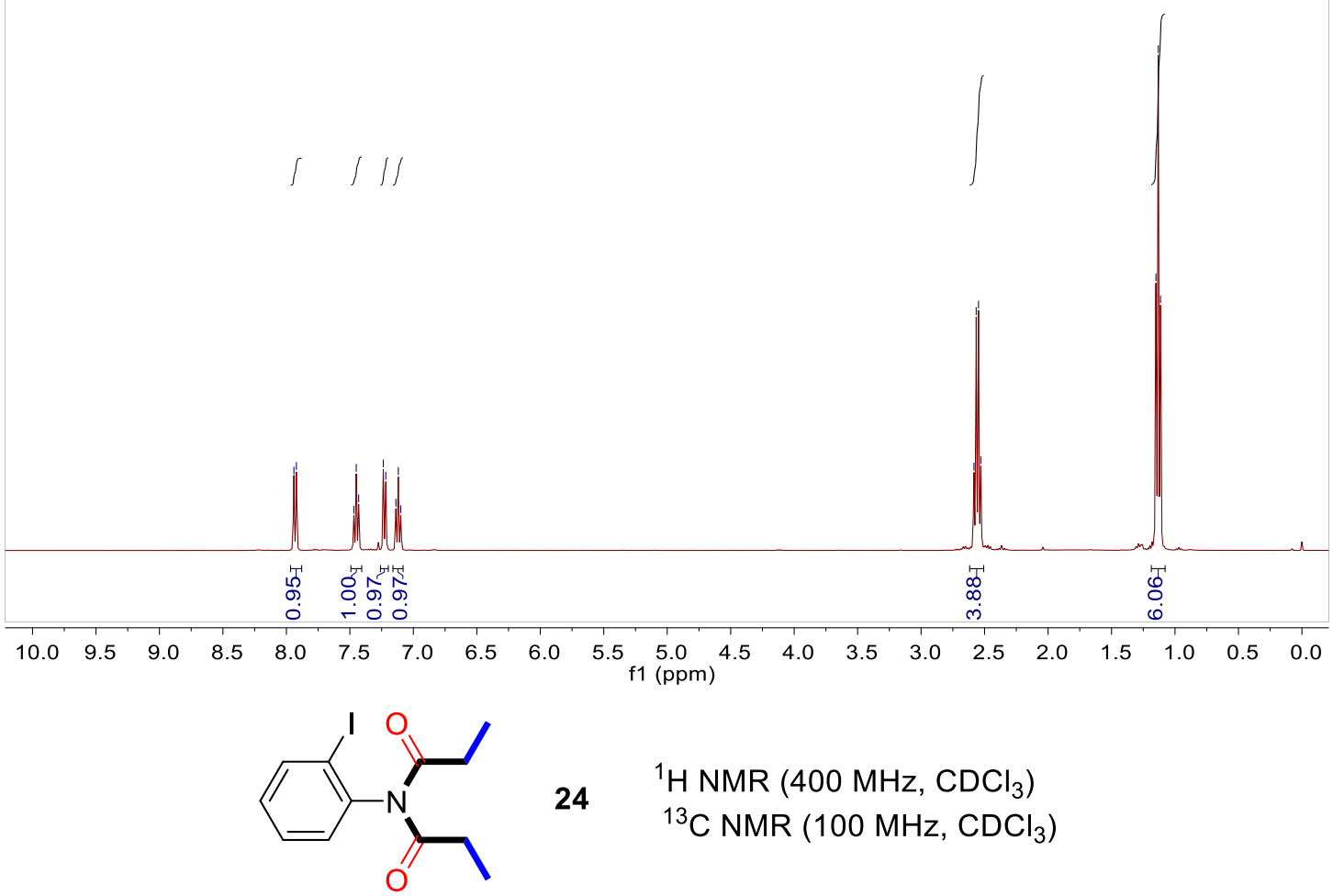

$24{ }^{1} \mathrm{H}$ NMR $\left(400 \mathrm{MHz}, \mathrm{CDCl}_{3}\right)$

${ }^{13} \mathrm{C} \mathrm{NMR}\left(100 \mathrm{MHz}, \mathrm{CDCl}_{3}\right)$

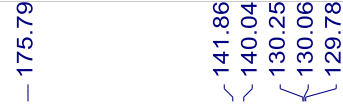

\begin{tabular}{l}
$\infty$ \\
$\infty$ \\
$\stackrel{0}{\circ}$ \\
\hline
\end{tabular}

\begin{tabular}{ll} 
N & \multirow{2}{*}{} \\
లె & $\infty$ \\
1 & 1
\end{tabular}

C13CPD CDCI3 \{D:INMR400IDNL0604\} nmr-new 58

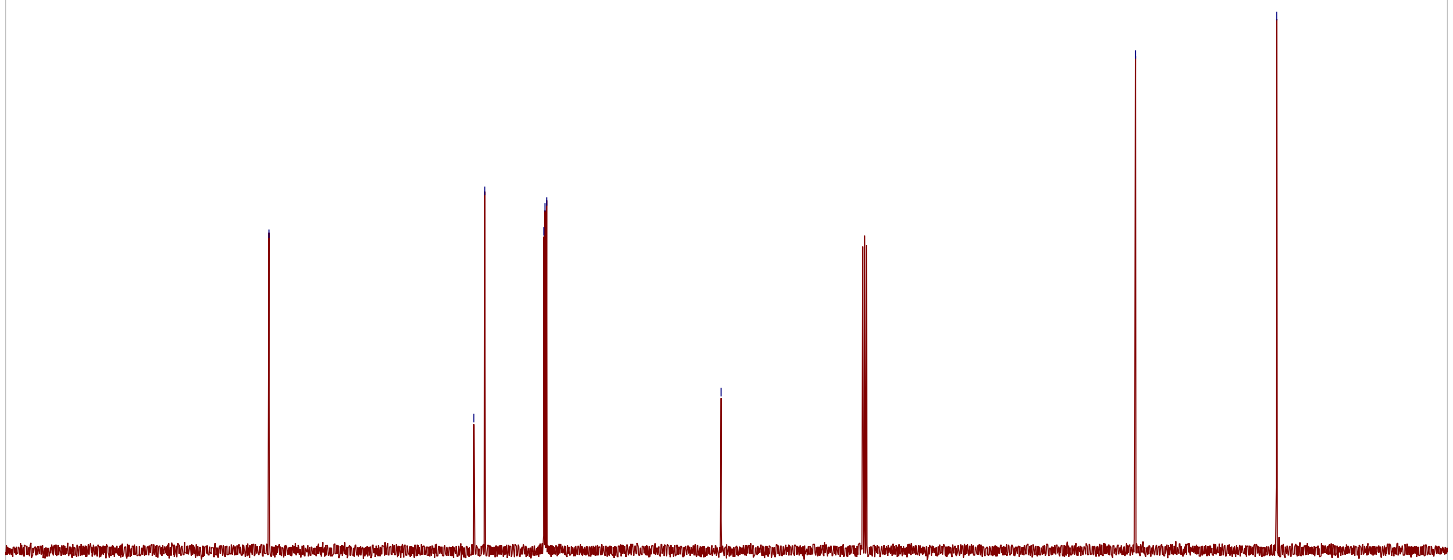

$\begin{array}{llllllllllllllllllllllll}210 & 200 & 190 & 180 & 170 & 160 & 150 & 140 & 130 & 120 & 110 & 100 & 90 & 80 & 70 & 60 & 50 & 40 & 30 & 20 & 10 & 0 & -10\end{array}$ f1 (ppm) 
csk-3-5-5.10.fid

PROTON CDCI3 \{D:INMR400ldnl0604\} nmr-new 7
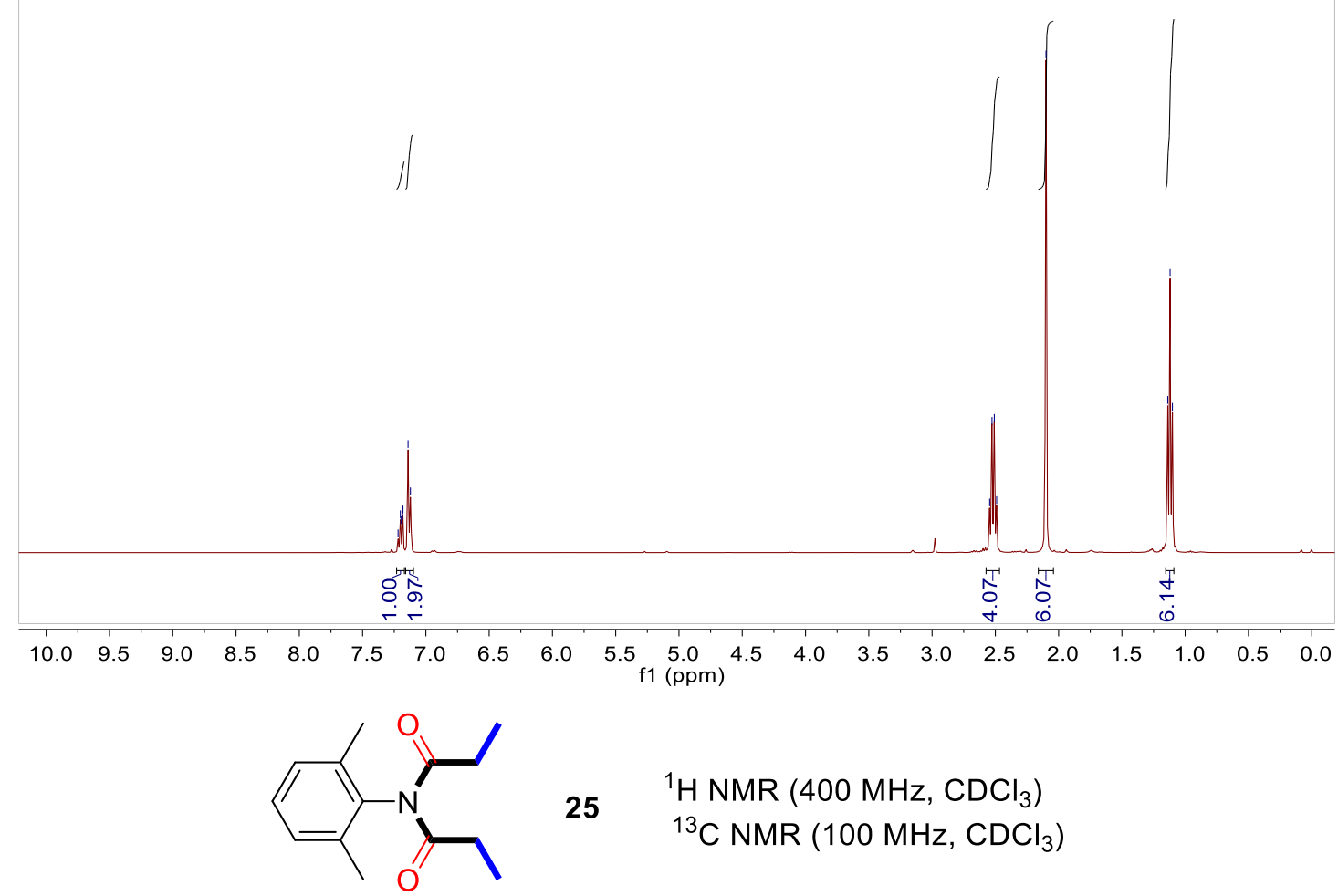

$25 \quad{ }^{1} \mathrm{H}$ NMR $\left(400 \mathrm{MHz}, \mathrm{CDCl}_{3}\right)$

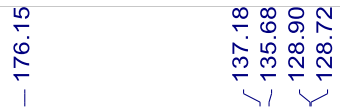

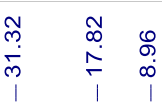

C13CPD CDCI3 \{D:INMR400Idnl0604\} nmr-new 7

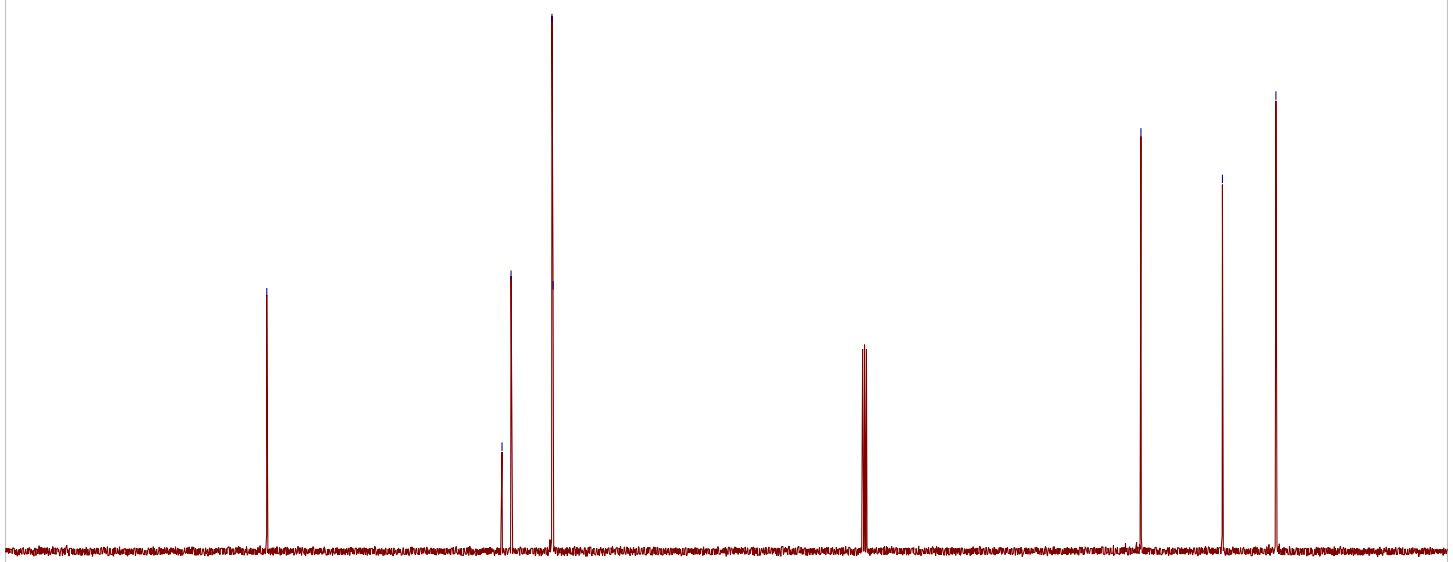

$\begin{array}{lllllllllllllllllllllll}210 & 200 & 190 & 180 & 170 & 160 & 150 & 140 & 130 & 120 & 110 & 100 & 90 & 80 & 70 & 60 & 50 & 40 & 30 & 20 & 10 & 0 & -10\end{array}$ 
csk-3-5-15.10.fid

PROTON CDCI3 \{D:INMR400Idnl0604\} nmr-new 31
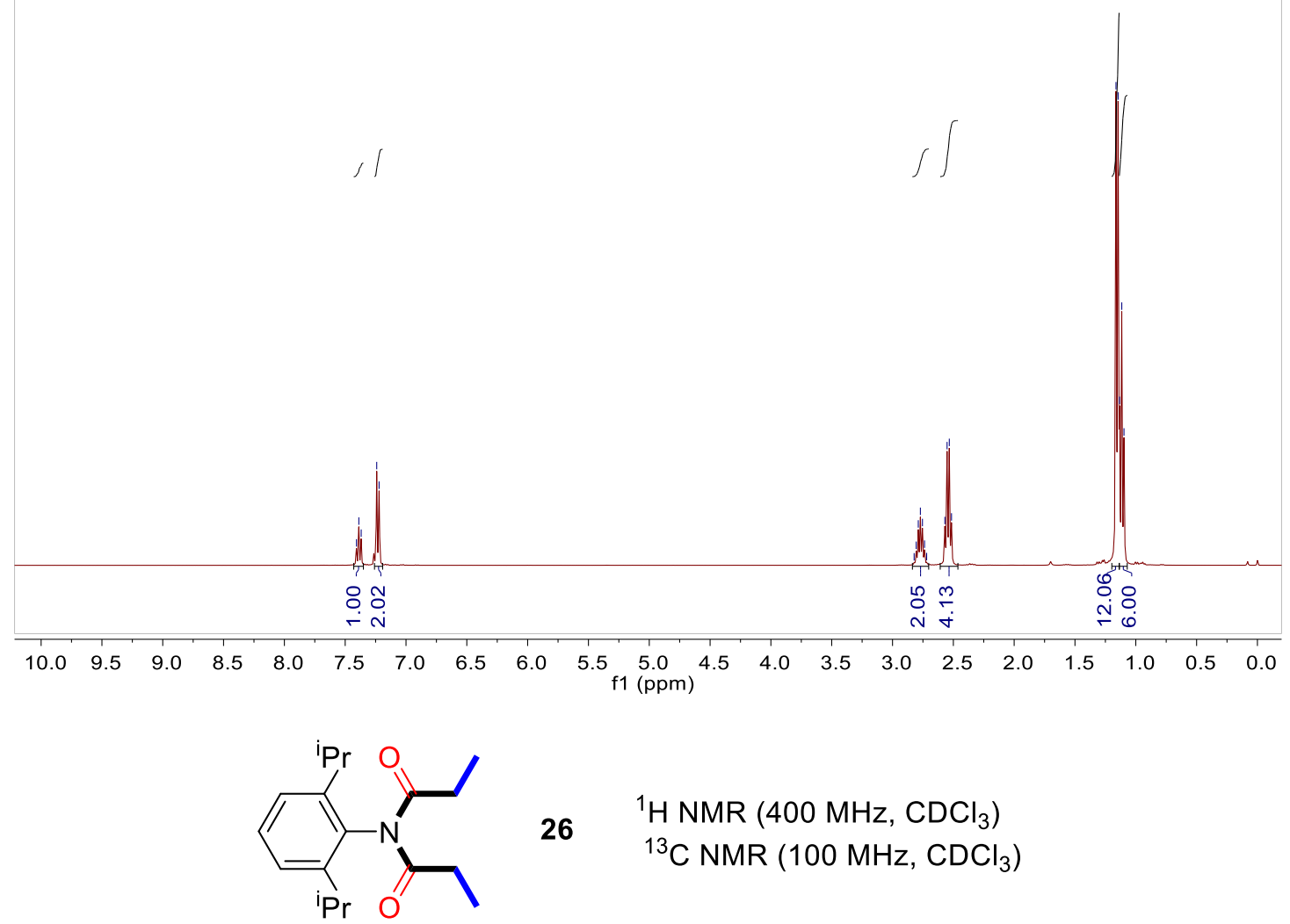

$26{ }^{1} \mathrm{H}$ NMR $\left(400 \mathrm{MHz}, \mathrm{CDCl}_{3}\right)$

${ }^{13} \mathrm{C}$ NMR $\left(100 \mathrm{MHz}, \mathrm{CDCl}_{3}\right)$

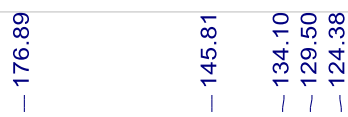

ํำ

$\dot{m} \stackrel{\infty}{\sim} \stackrel{\text { N }}{1}$

csk-3-5-15.11.fid

C13CPD CDCl3 \{D:INMR400ldn10604\} nmr-new 31

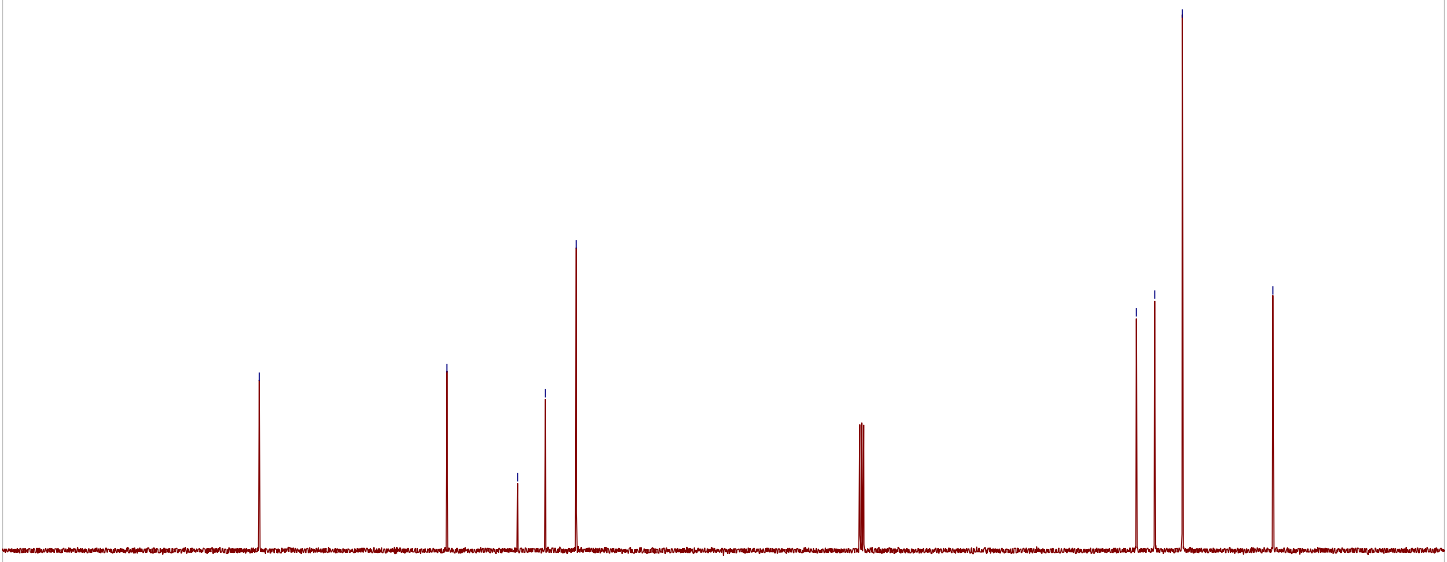

$\begin{array}{lllllllllllll}210 & 200 & 190 & 180 & 170 & 160 & 150 & 140 & 130 & 120 & 110 & 100 & 90\end{array}$ 
csk-2-101-6.10.fid

PROTON CDCI3 \{D:INMR400IDNL0604\} nmr-new 51

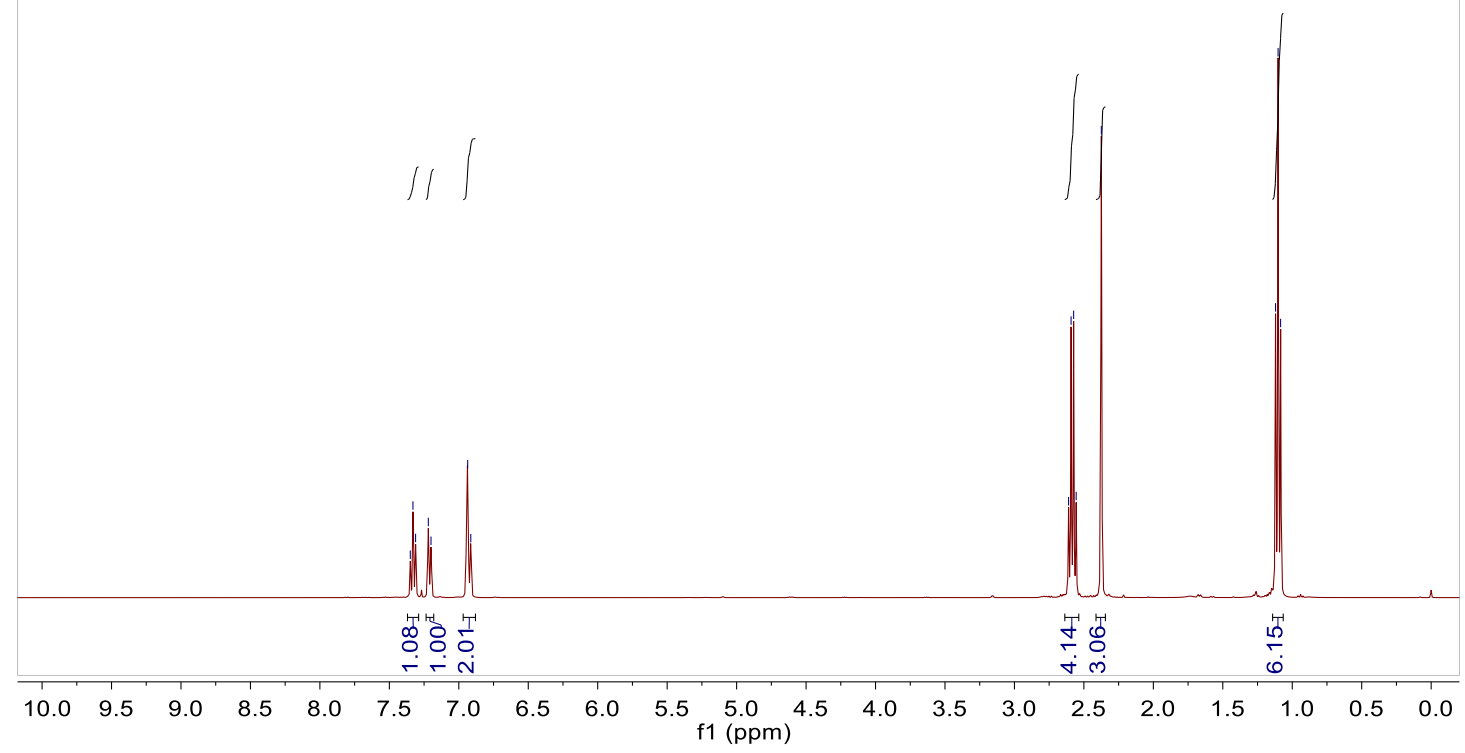

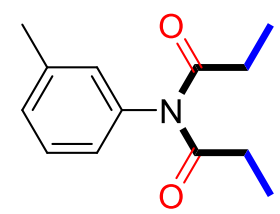

$\frac{1}{\stackrel{1}{0}}$

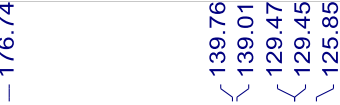

sk-2-101-6.11 fid

C13CPD CDCI3 \{D:INMR400IDNL0604\} nmr-new 51

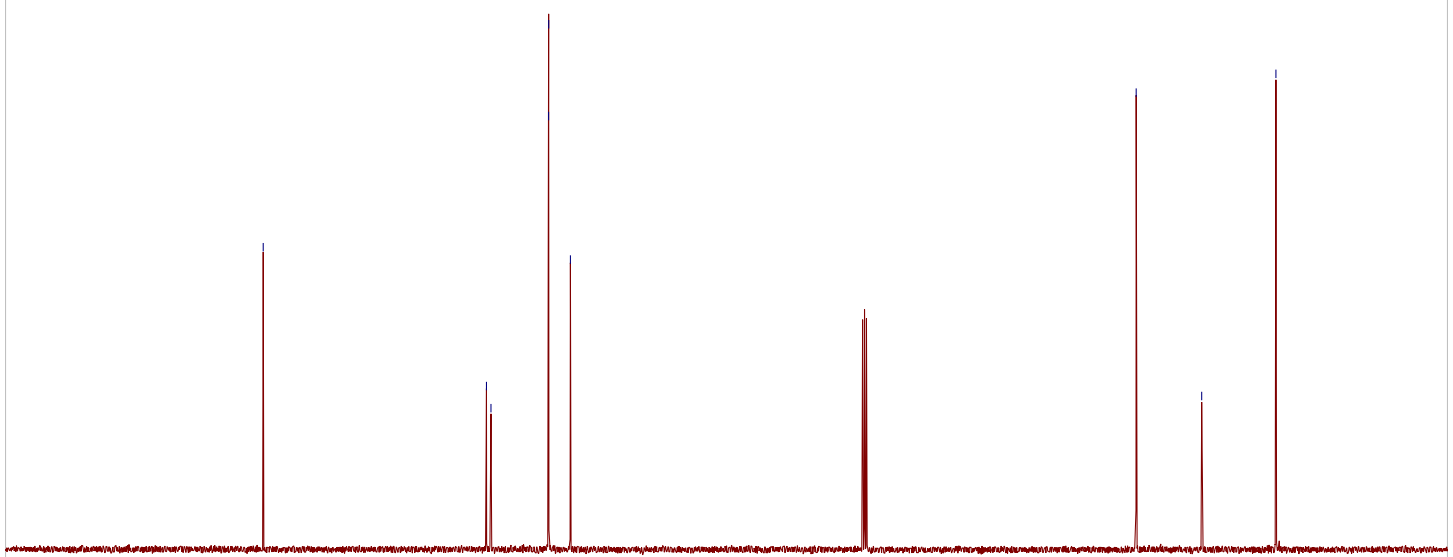

$\begin{array}{llllllllllllllllllllllll}210 & 200 & 190 & 180 & 170 & 160 & 150 & 140 & 130 & 120 & 110 & 100 & 90 & 80 & 70 & 60 & 50 & 40 & 30 & 20 & 10 & 0 & -10\end{array}$ $\mathrm{f1}(\mathrm{ppm})$ 


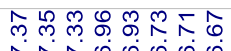

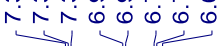

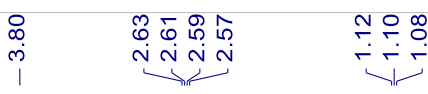

csk-3-19-2.10.fid

PROTON CDCI3 \{D:INMR400IDNL0604\} nmr-new 42
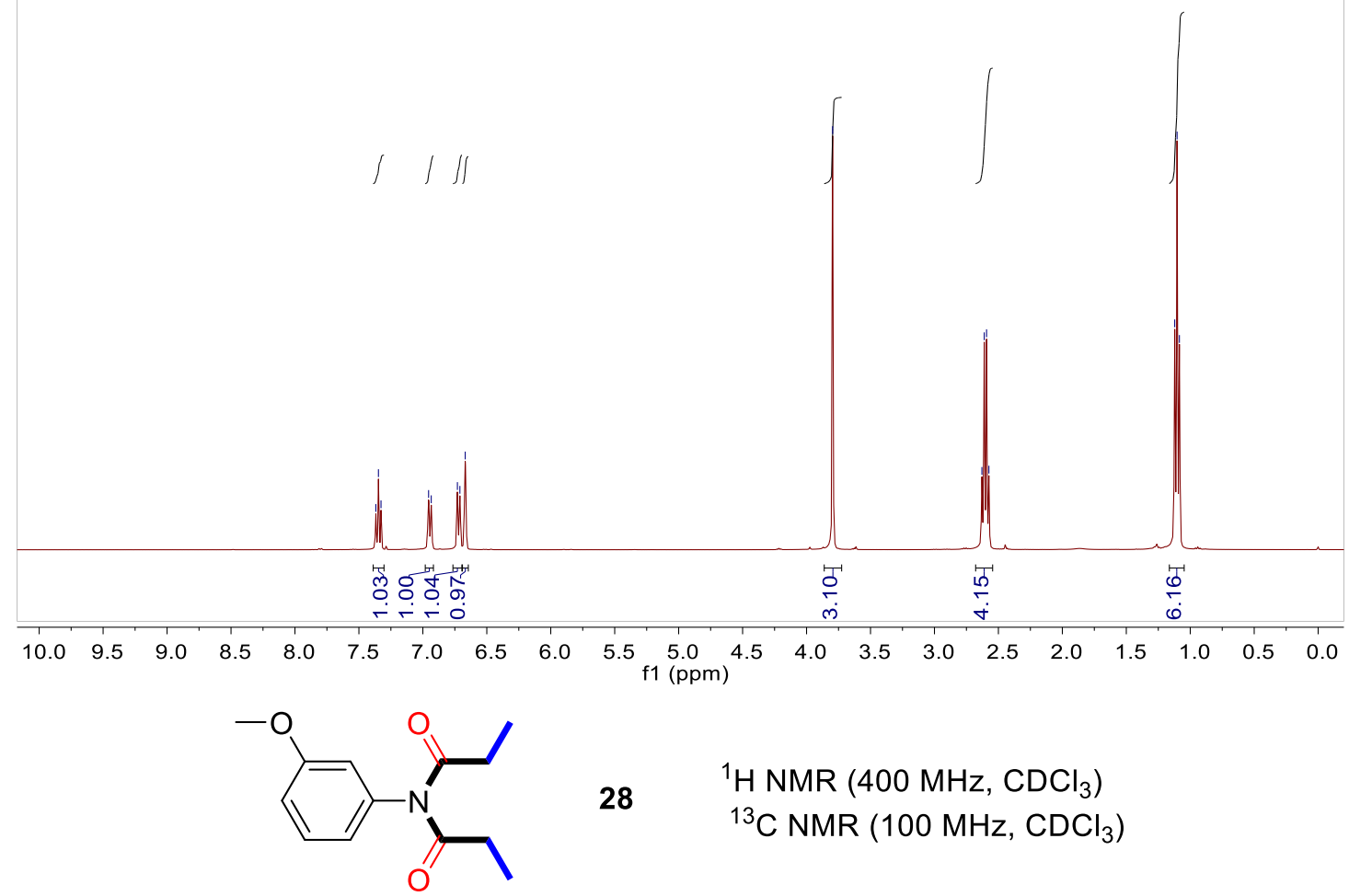

28

${ }^{1} \mathrm{H}$ NMR (400 MHz, $\left.\mathrm{CDCl}_{3}\right)$

${ }^{13} \mathrm{C}$ NMR (100 MHz, $\mathrm{CDCl}_{3}$ )

\begin{tabular}{|c|c|c|c|}
\hline $\begin{array}{ll}\stackrel{0}{L} & \infty \\
0 & 0 \\
0 & 0 \\
0 & 0 \\
& 0 \\
& 1\end{array}$ & 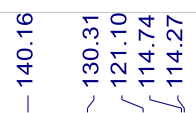 & 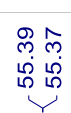 & $\stackrel{\infty}{\stackrel{\infty}{-}}$ \\
\hline
\end{tabular}

csk-3-19-2.11.fid

C13CPD CDCI3 \{D:INMR400IDNL0604\} nmr-new 42

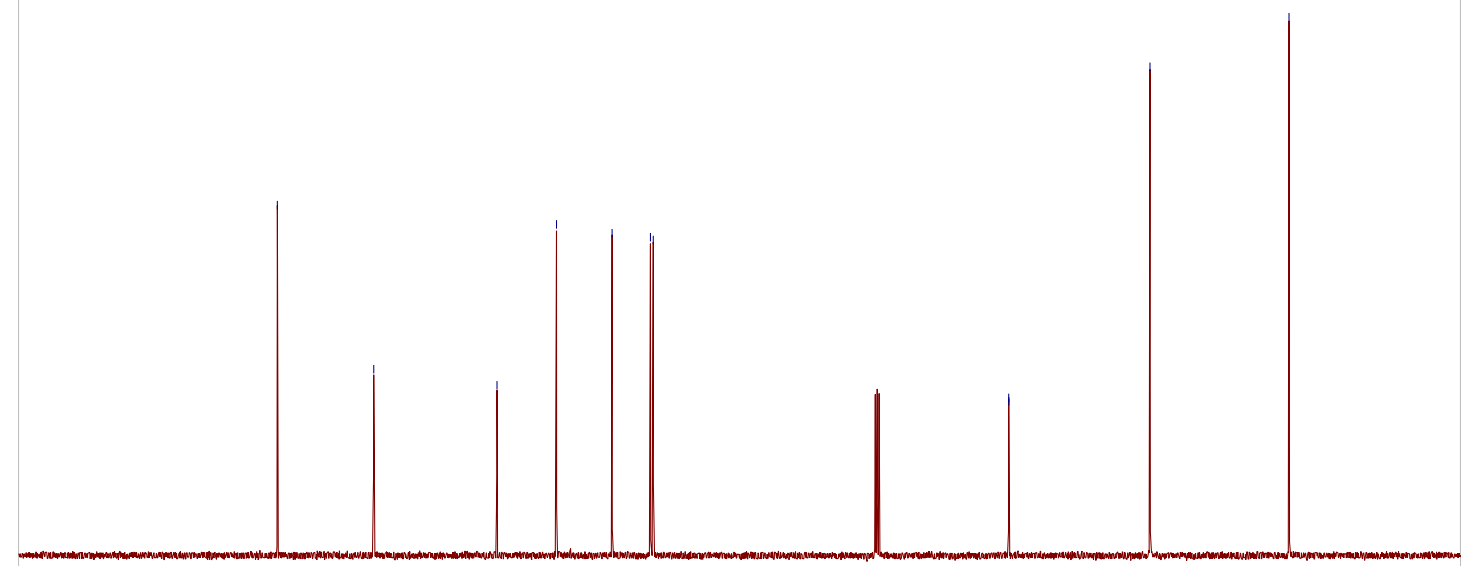

$\begin{array}{lllllllllllllllllllllll}210 & 200 & 190 & 180 & 170 & 160 & 150 & 140 & 130 & 120 & 110 & 100 & 90 & 80 & 70 & 60 & 50 & 40 & 30 & 20 & 10 & 0 & -10\end{array}$ 
csk-3-19-3.10.fid

PROTON CDCI3 \{D:INMR400IDNL0604\} nmr-new 43

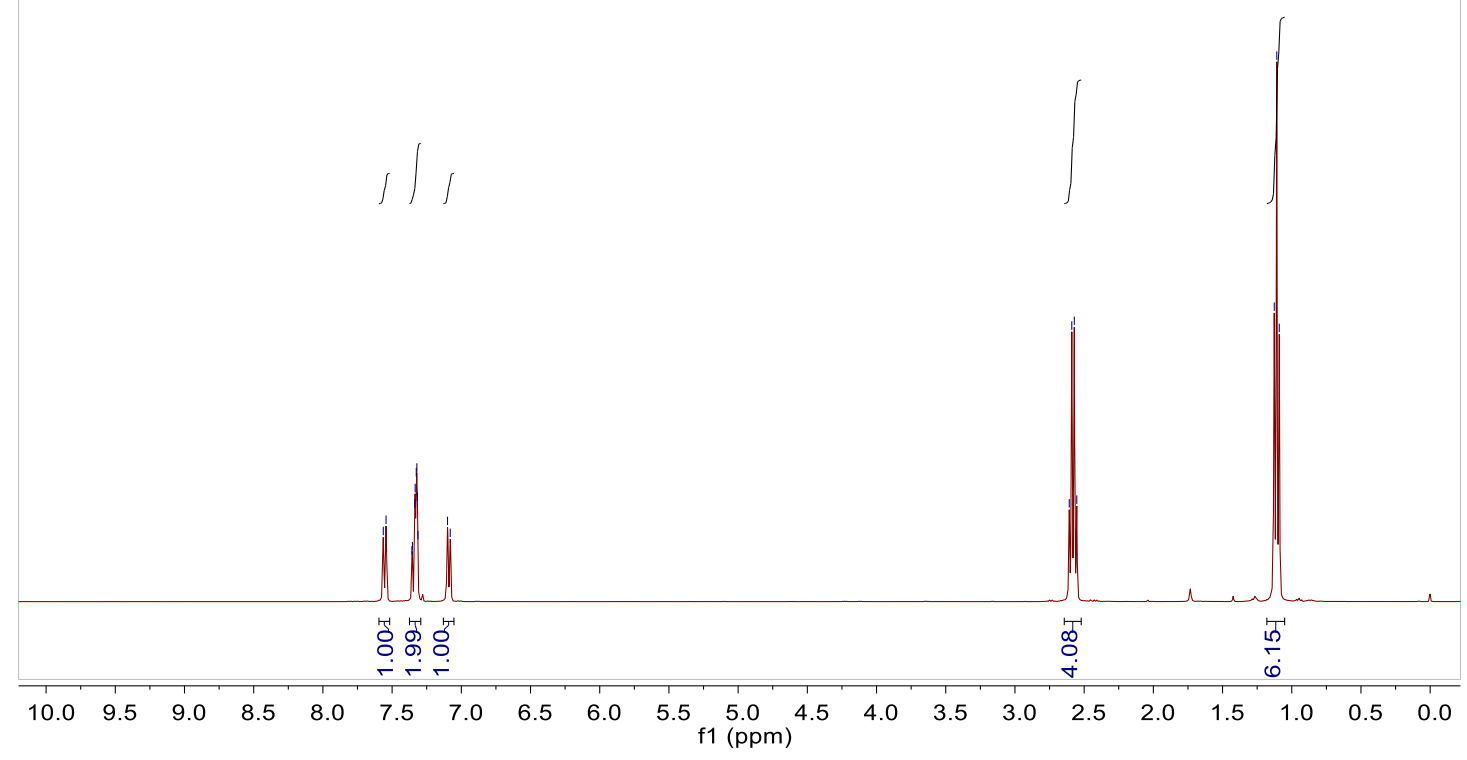

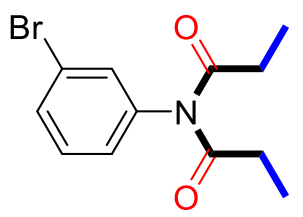

${ }^{1} \mathrm{H}$ NMR $\left(400 \mathrm{MHz}, \mathrm{CDCl}_{3}\right)$

${ }^{13} \mathrm{C}$ NMR $\left(100 \mathrm{MHz}, \mathrm{CDCl}_{3}\right)$

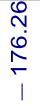

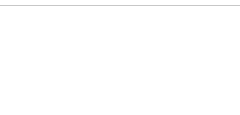

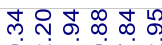

守

csk-3-19-3.11.fid

C13CPD CDCI3 \{D:INMR400IDNL0604\} nmr-new 43

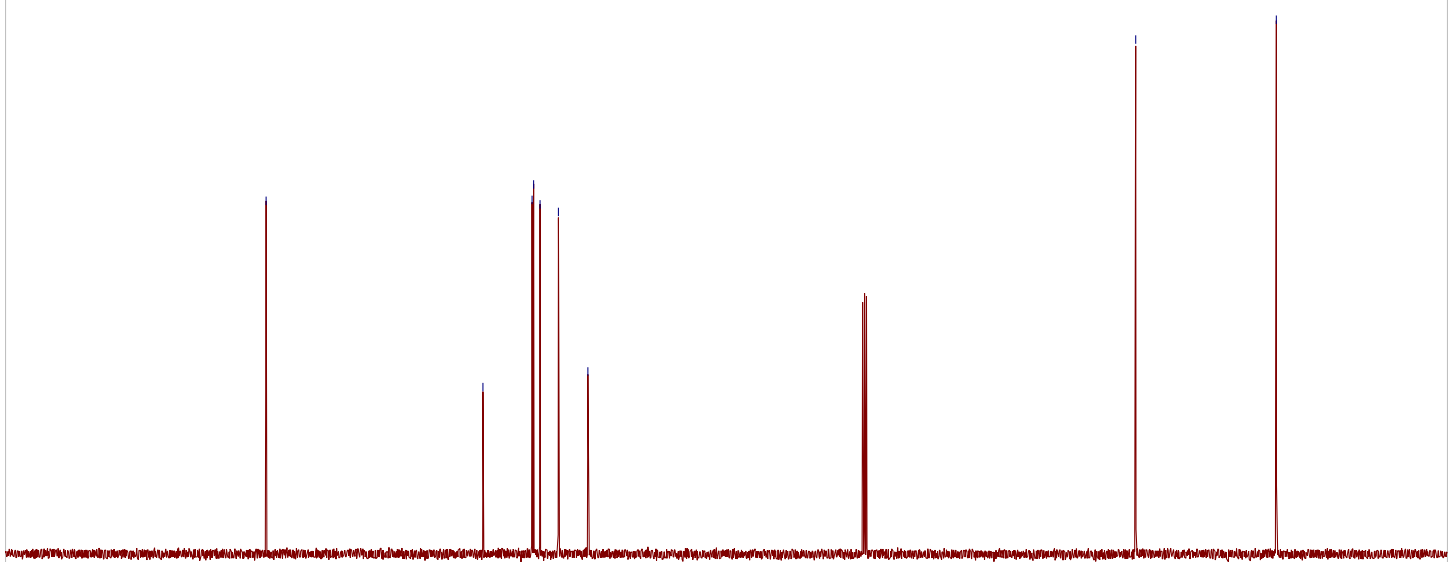

$\begin{array}{lllllllllllllllllllllll}210 & 200 & 190 & 180 & 170 & 160 & 150 & 140 & 130 & 120 & 110 & 100 & 90 & 80 & 70 & 60 & 50 & 40 & 30 & 20 & 10 & 0 & -10\end{array}$ 
csk-3-5-12.10.fid

PROTON CDCI3 \{D:INMR400IDNL0604\} nmr-new 57
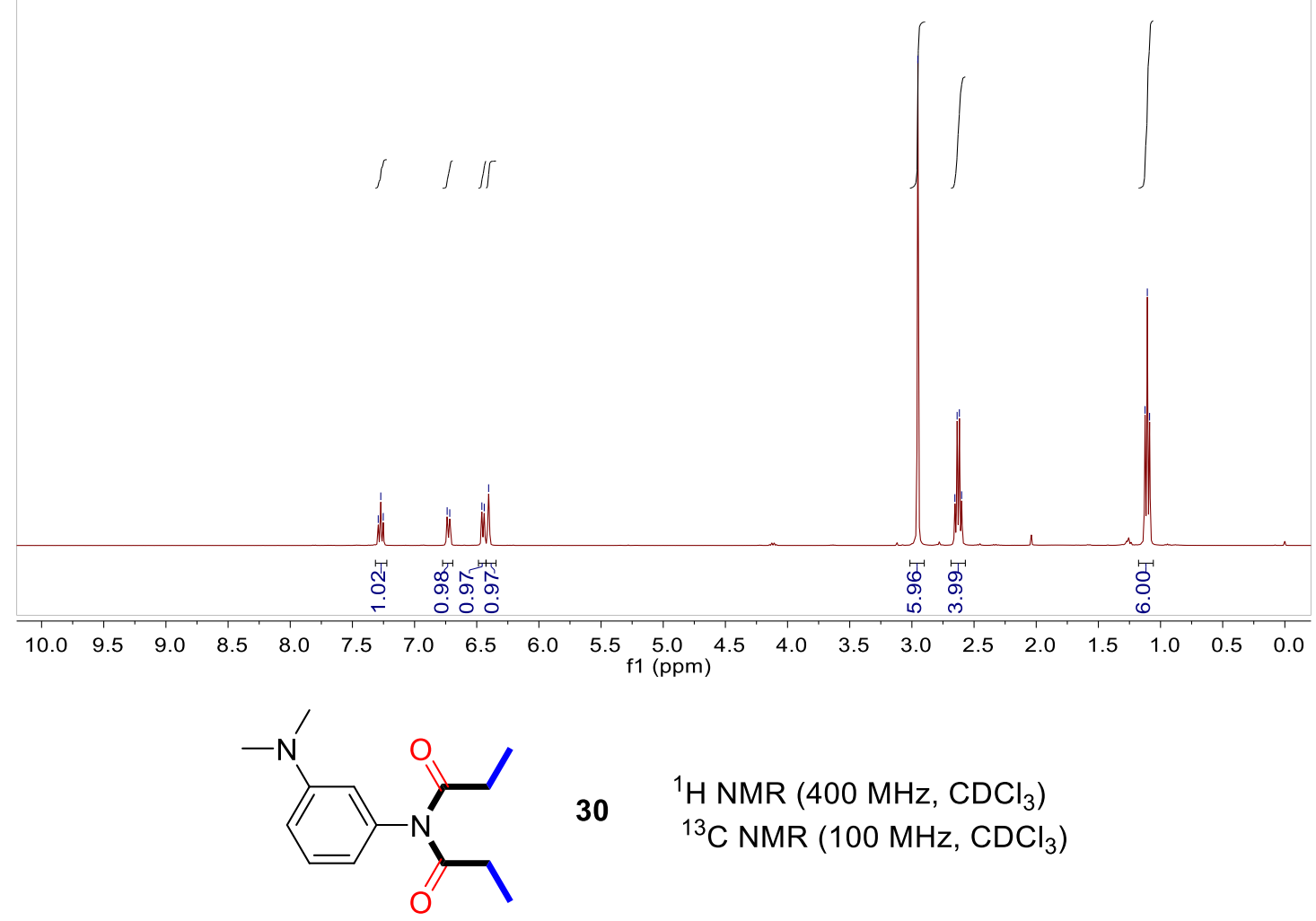

30

${ }^{1} \mathrm{H}$ NMR $\left(400 \mathrm{MHz}, \mathrm{CDCl}_{3}\right)$

${ }^{13} \mathrm{C} \mathrm{NMR}\left(100 \mathrm{MHz}, \mathrm{CDCl}_{3}\right)$

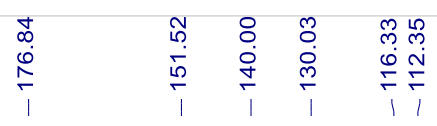

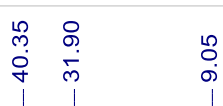

csk-3-5-12.11.fid

C13CPD CDCI3 \{D:INMR400IDNL0604\} nmr-new 57

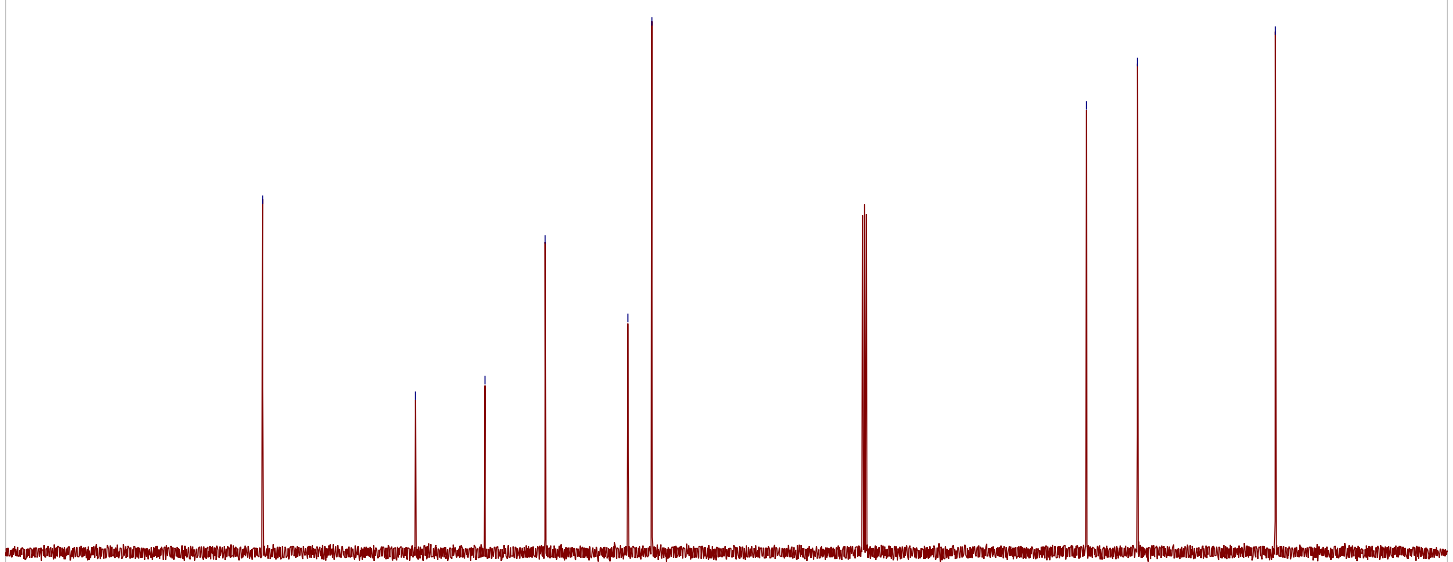

$\begin{array}{lllllllllllllllllllllll}210 & 200 & 190 & 180 & 170 & 160 & 150 & 140 & 130 & 120 & 110 & 100 & 90 & 80 & 70 & 60 & 50 & 40 & 30 & 20 & 10 & 0 & -10\end{array}$ 


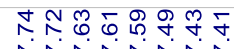 \\ NANNNN}

csk-3-19-1.10.fid

PROTON CDCI3 \{D:INMR400IDNL0604\} nmr-new 41
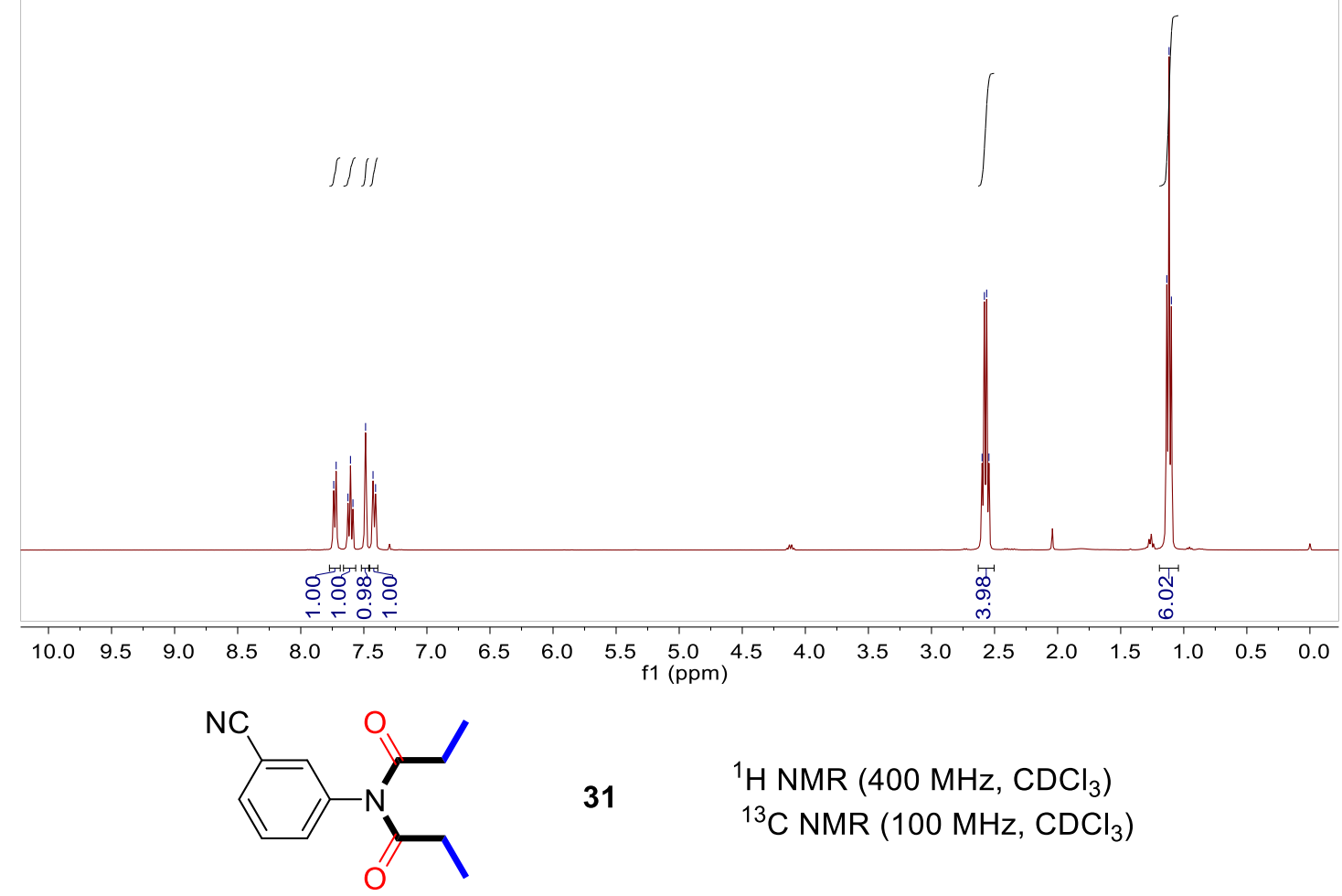

$31 \quad{ }^{1} \mathrm{H} \mathrm{NMR}\left(400 \mathrm{MHz}, \mathrm{CDCl}_{3}\right)$

${ }^{13} \mathrm{C}$ NMR $\left(100 \mathrm{MHz}, \mathrm{CDCl}_{3}\right)$

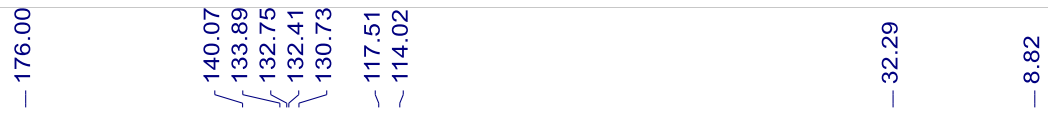

csk-3-19-1.11.fid

C13CPD CDCI3 \{D:INMR400IDNL0604\} nmr-new 41

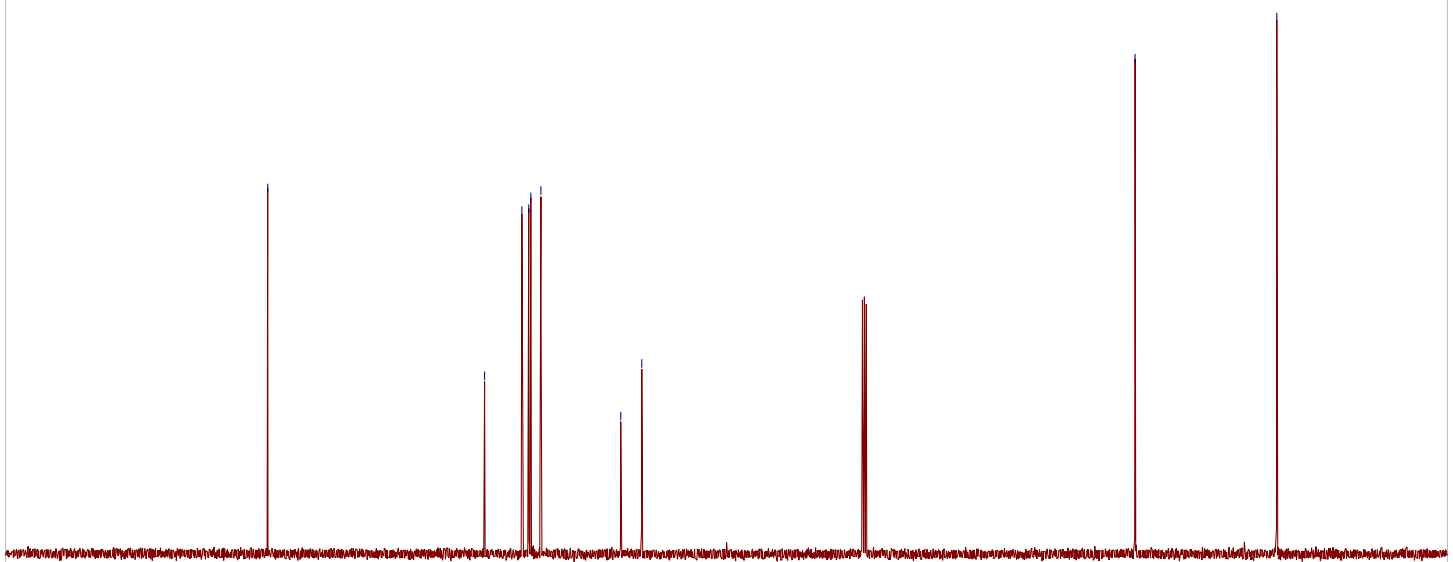

$\begin{array}{lllllllllllllllllllllll}210 & 200 & 190 & 180 & 170 & 160 & 150 & 140 & 130 & 120 & 110 & 100 & 90 & 80 & 70 & 60 & 50 & 40 & 30 & 20 & 10 & 0 & -10\end{array}$ 
csk-3-5-2.10.fid

PROTON CDCI3 \{D:INMR400ldnl0604\} nmr-new 4

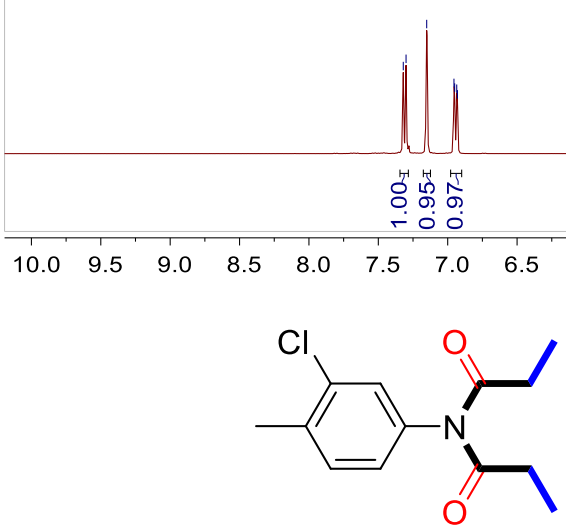

$\frac{1}{\check{1}}$

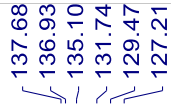

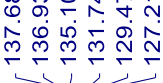

32
${ }^{1} \mathrm{H}$ NMR $\left(400 \mathrm{MHz}, \mathrm{CDCl}_{3}\right)$

${ }^{13} \mathrm{C} \mathrm{NMR}\left(100 \mathrm{MHz}, \mathrm{CDCl}_{3}\right)$

sk-3-5-2.11.fid

C13CPD CDCI3 \{D:INMR400ldnl0604\} nmr-new 4

$\begin{array}{lll}m & \circ & \circ \\ & \stackrel{\infty}{\circ} & \infty \\ 1 & 1 & 1\end{array}$

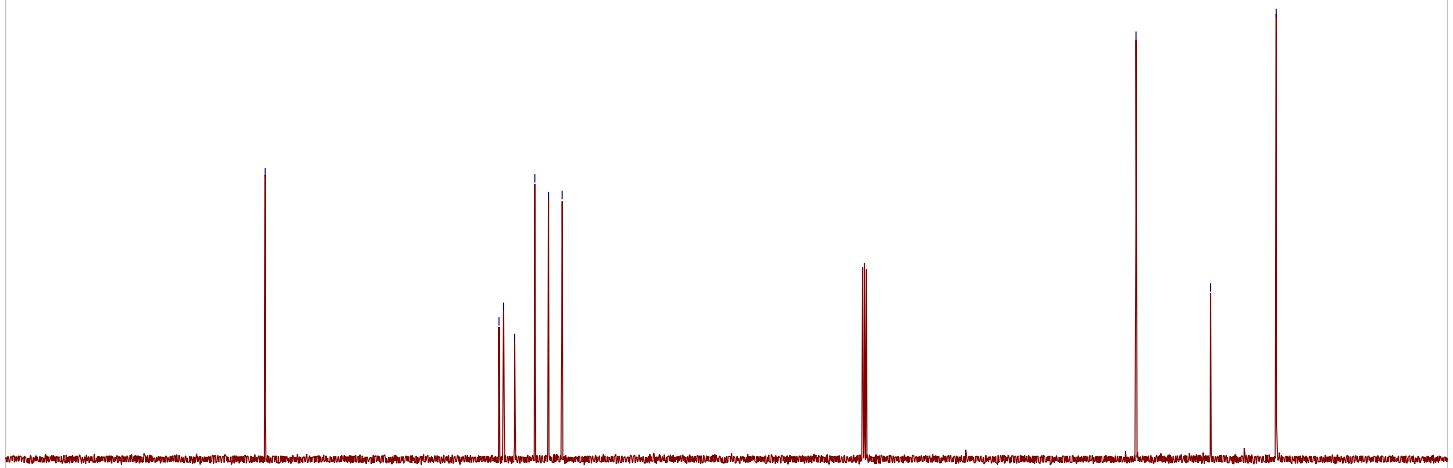

$\begin{array}{lllllllllllllllllllllll}210 & 200 & 190 & 180 & 170 & 160 & 150 & 140 & 130 & 120 & 110 & 100 & 90 & 80 & 70 & 60 & 50 & 40 & 30 & 20 & 10 & 0 & -10\end{array}$ 


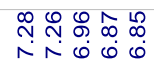

र

csk-3-19-8.10.fid

PROTON CDCI3 \{D:INMR400IDNL0604\} nmr-new 11

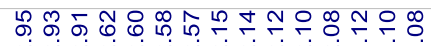

ง

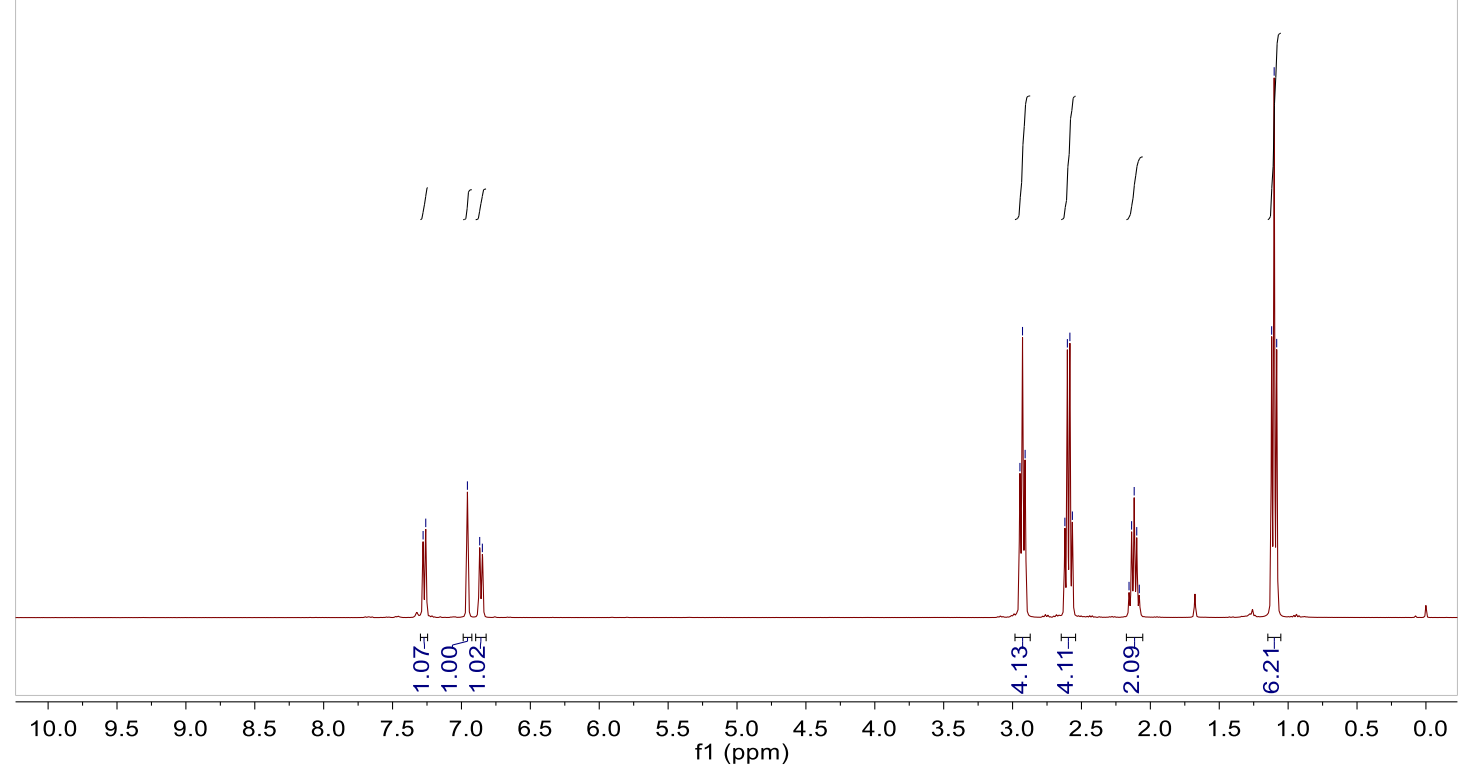

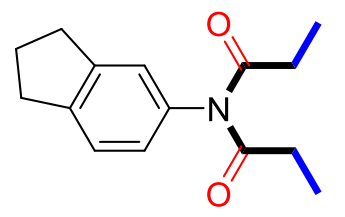

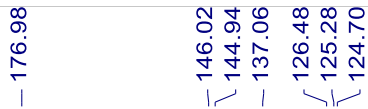

33

${ }^{1} \mathrm{H}$ NMR $\left(400 \mathrm{MHz}, \mathrm{CDCl}_{3}\right)$

${ }^{13} \mathrm{C} \mathrm{NMR}\left(100 \mathrm{MHz}, \mathrm{CDCl}_{3}\right)$

csk-3-19-8.11.fid

C13CPD CDCI3 \{D:INMR400IDNL0604\} nmr-new 11

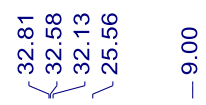

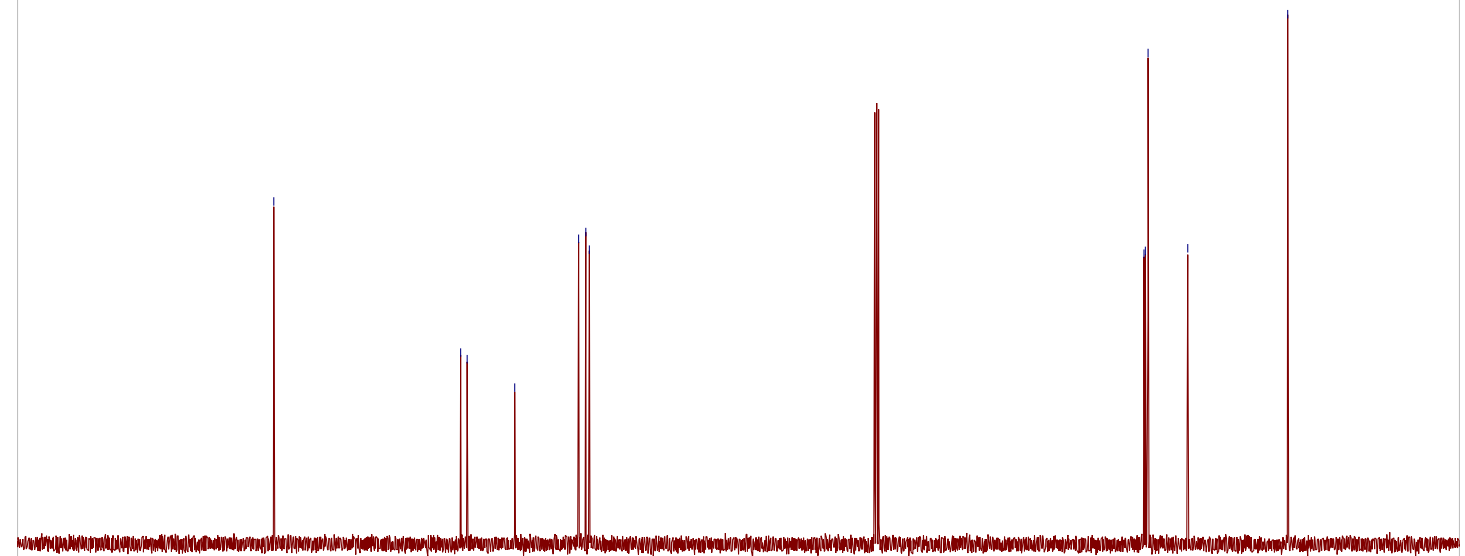

$\begin{array}{lllllllllllllllllllllll}210 & 200 & 190 & 180 & 170 & 160 & 150 & 140 & 130 & 120 & 110 & 100 & 90 & 80 & 70 & 60 & 50 & 40 & 30 & 20 & 10 & 0 & -10\end{array}$ f1 (ppm) 
csk-2-101-7.10.fid

PROTON CDCI3 \{D:INMR400IDNL0604\} nmr-new 52
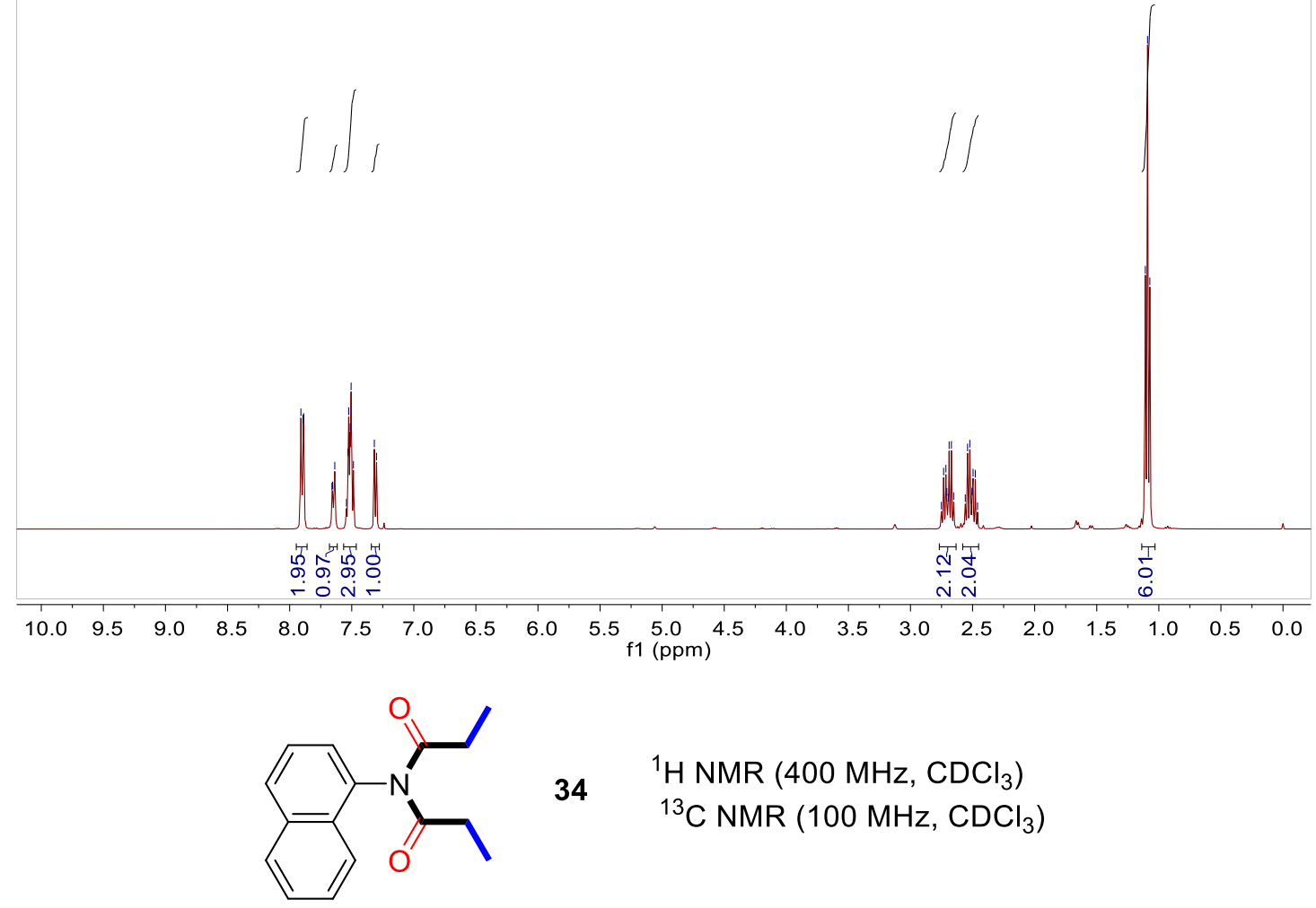

34

${ }^{1} \mathrm{H} \mathrm{NMR}\left(400 \mathrm{MHz}, \mathrm{CDCl}_{3}\right)$

${ }^{13} \mathrm{C} \mathrm{NMR}\left(100 \mathrm{MHz}, \mathrm{CDCl}_{3}\right)$

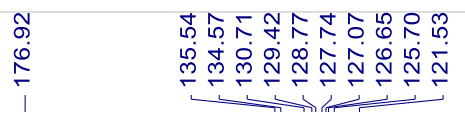

$\begin{array}{ll}\stackrel{\infty}{n} & m \\ i & 0\end{array}$

csk-2-101-7.11.fid

C13CPD CDCI3 \{D:INMR400IDNL0604\} nmr-new 52

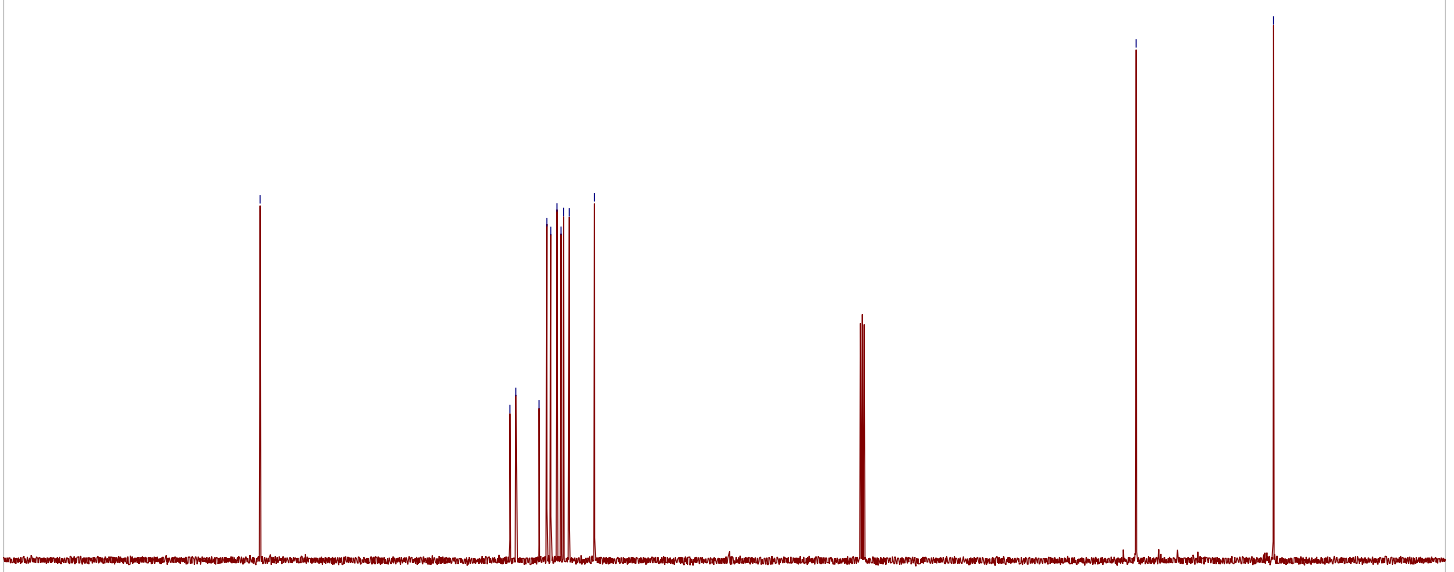

$\begin{array}{lllllllllllll}210 & 200 & 190 & 180 & 170 & 160 & 150 & 140 & 130 & 120 & 110 & 100 & 90\end{array}$ 
csk-3-5-4.10.fid

PROTON CDCl3 \{D:INMR400ldnl0604\} nmr-new 6
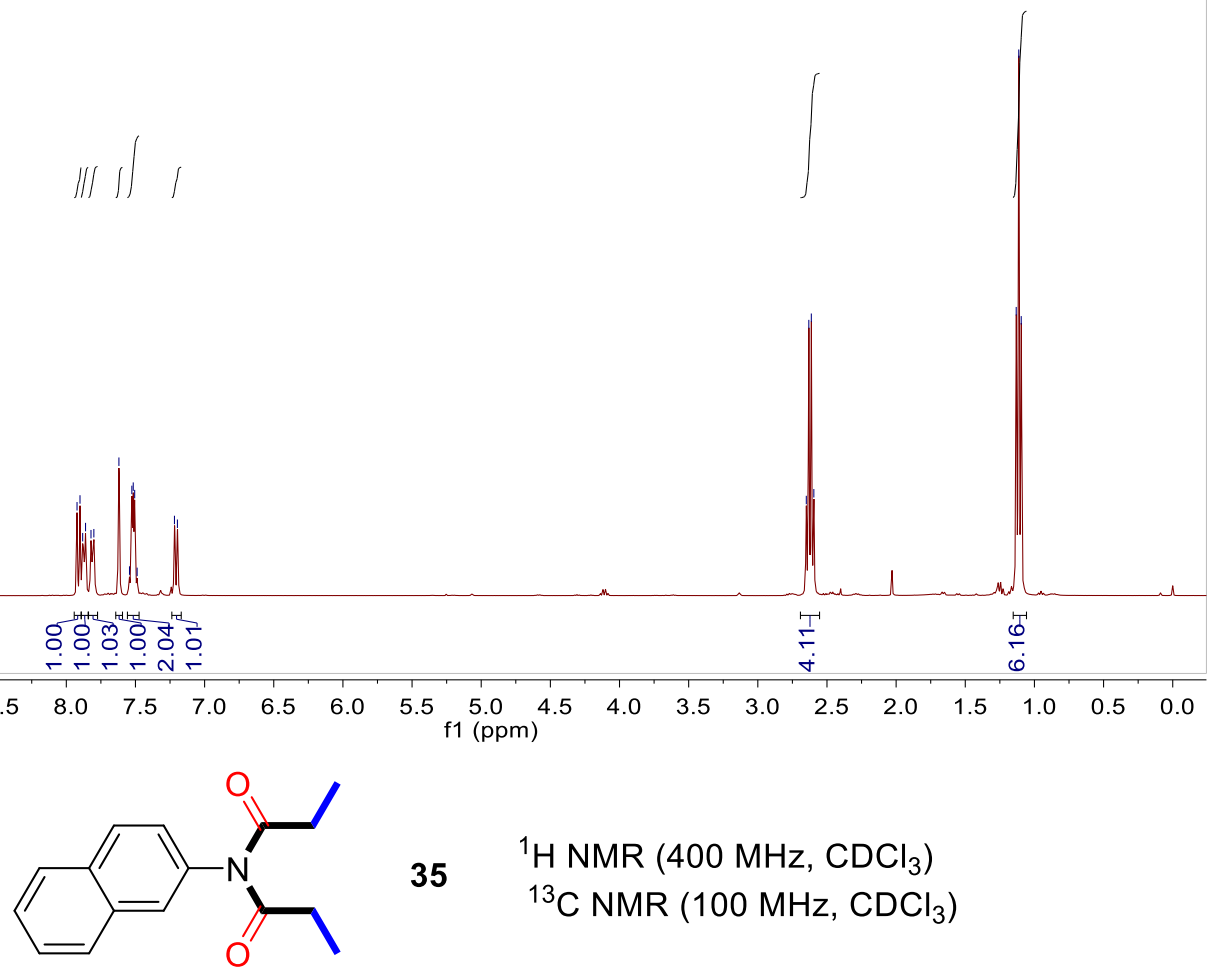

35

${ }^{1} \mathrm{H}$ NMR $\left(400 \mathrm{MHz}, \mathrm{CDCl}_{3}\right)$

${ }^{13} \mathrm{C}$ NMR (100 MHz, $\mathrm{CDCl}_{3}$ )

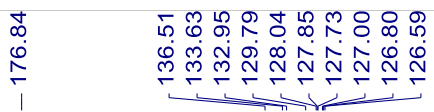

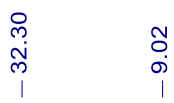

csk-3-5-4.11.fid

C13CPD CDCI3 \{D:INMR400ldnl0604\} nmr-new 6

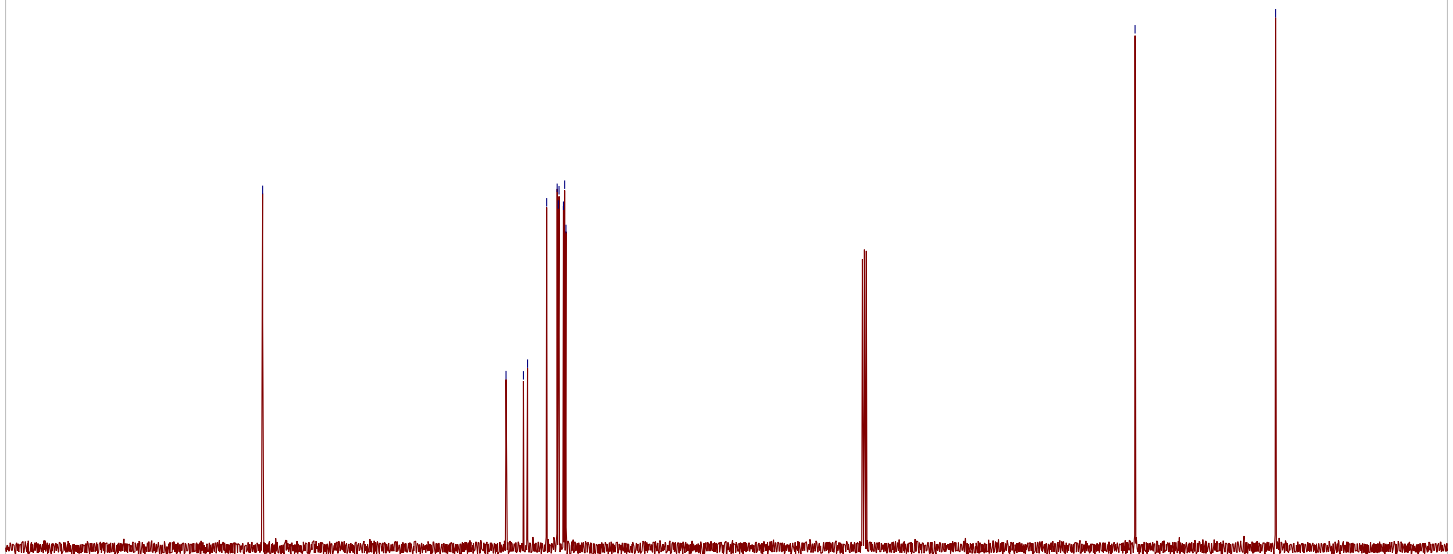

$\begin{array}{llllllllllllllllllllllll}210 & 200 & 190 & 180 & 170 & 160 & 150 & 140 & 130 & 120 & 110 & 100 & 90 & 80 & 70 & 60 & 50 & 40 & 30 & 20 & 10 & 0 & -10\end{array}$ f1 (ppm) 
csk-3-12-2.10.fid

PROTON CDCI3 \{D:INMR400ldnl0604\} nmr-new 3

\section{\|I川}
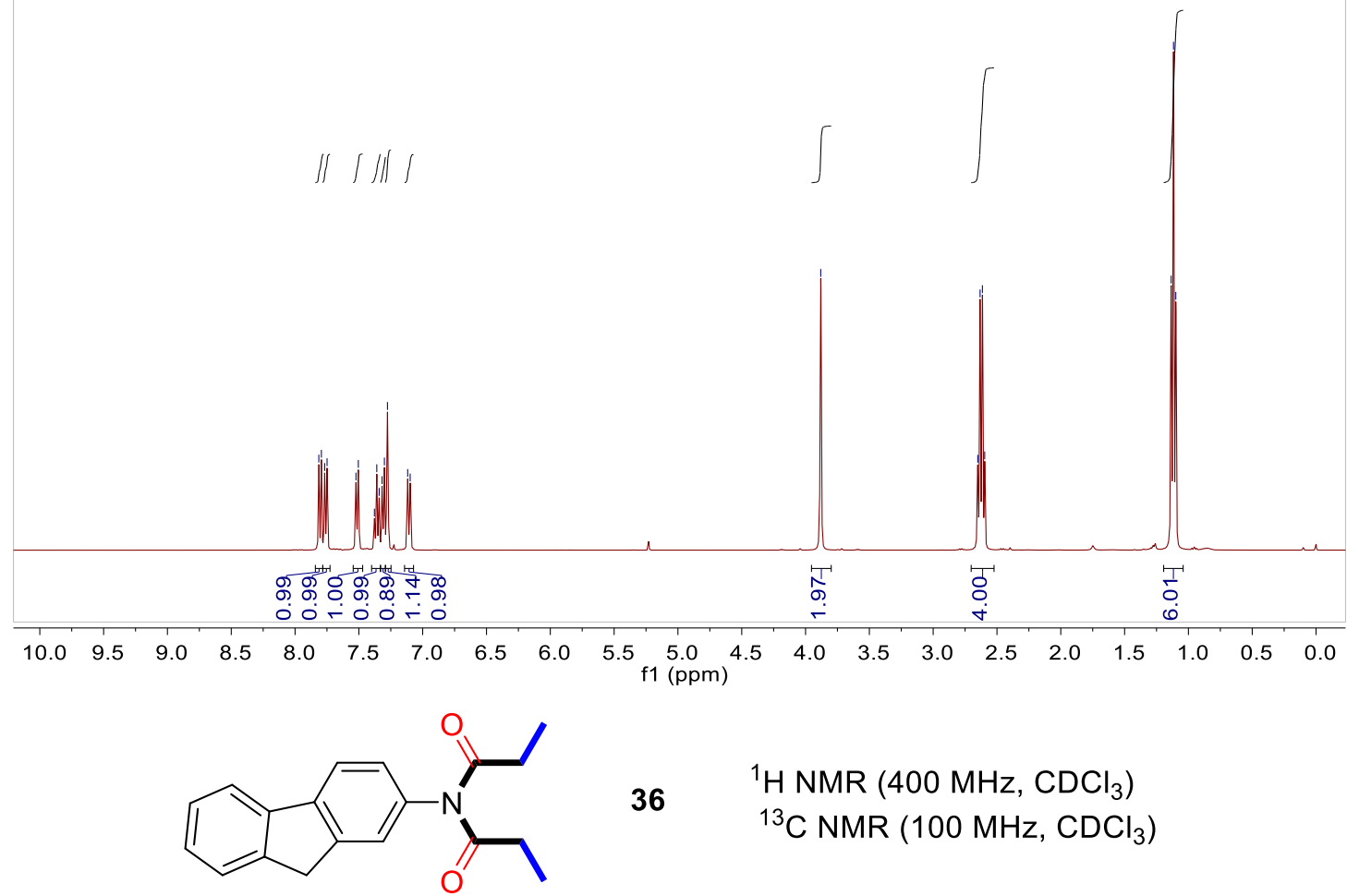

36

${ }^{1} \mathrm{H}$ NMR (400 MHz, $\mathrm{CDCl}_{3}$ )

${ }^{13} \mathrm{C}$ NMR (100 MHz, $\mathrm{CDCl}_{3}$ )

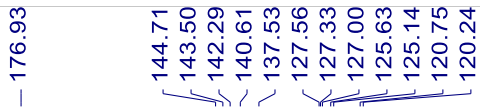

ฌ ్ำ

लंल

$\stackrel{0}{o}$

csk-3-12-2.11 fid

C13CPD CDCI3 \{D:INMR400ldnI0604\} nmr-new 3

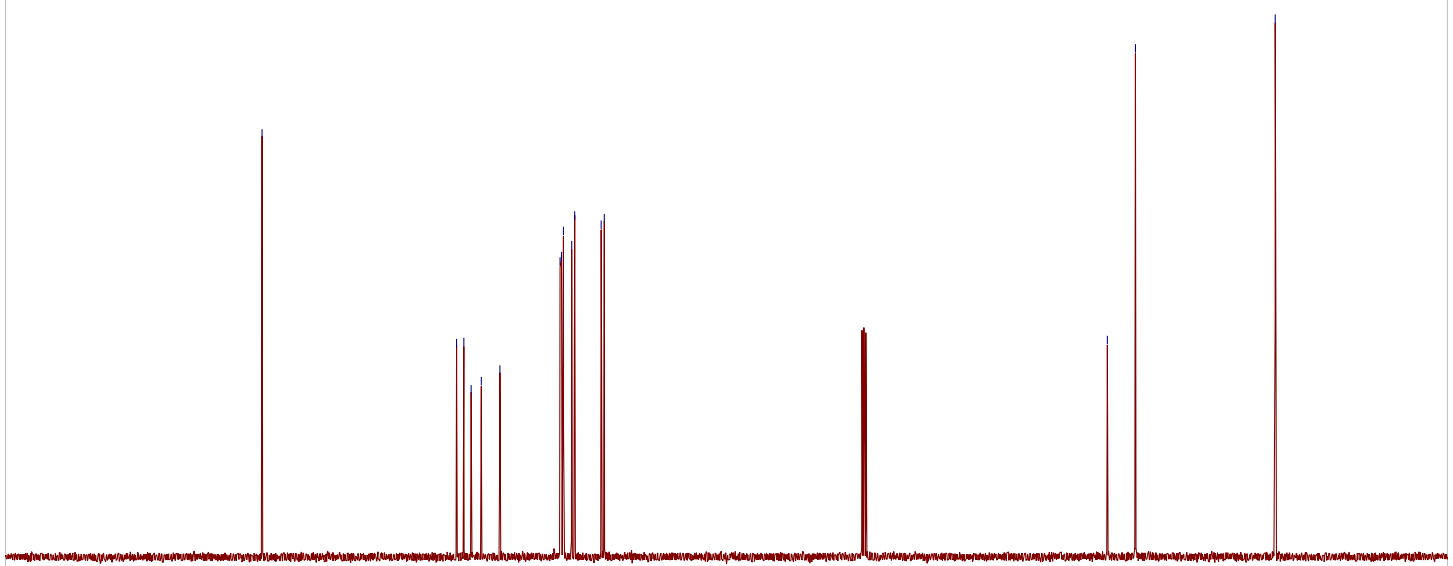

$\begin{array}{llllllllllllllllllllllll}210 & 200 & 190 & 180 & 170 & 160 & 150 & 140 & 130 & 120 & 110 & 100 & 90 & 80 & 70 & 60 & 50 & 40 & 30 & 20 & 10 & 0 & -10\end{array}$ 
sk-3-18-5.10.fid

PROTON CDCI3 \{D:INMR400ldnl0604\} nmr-new 6
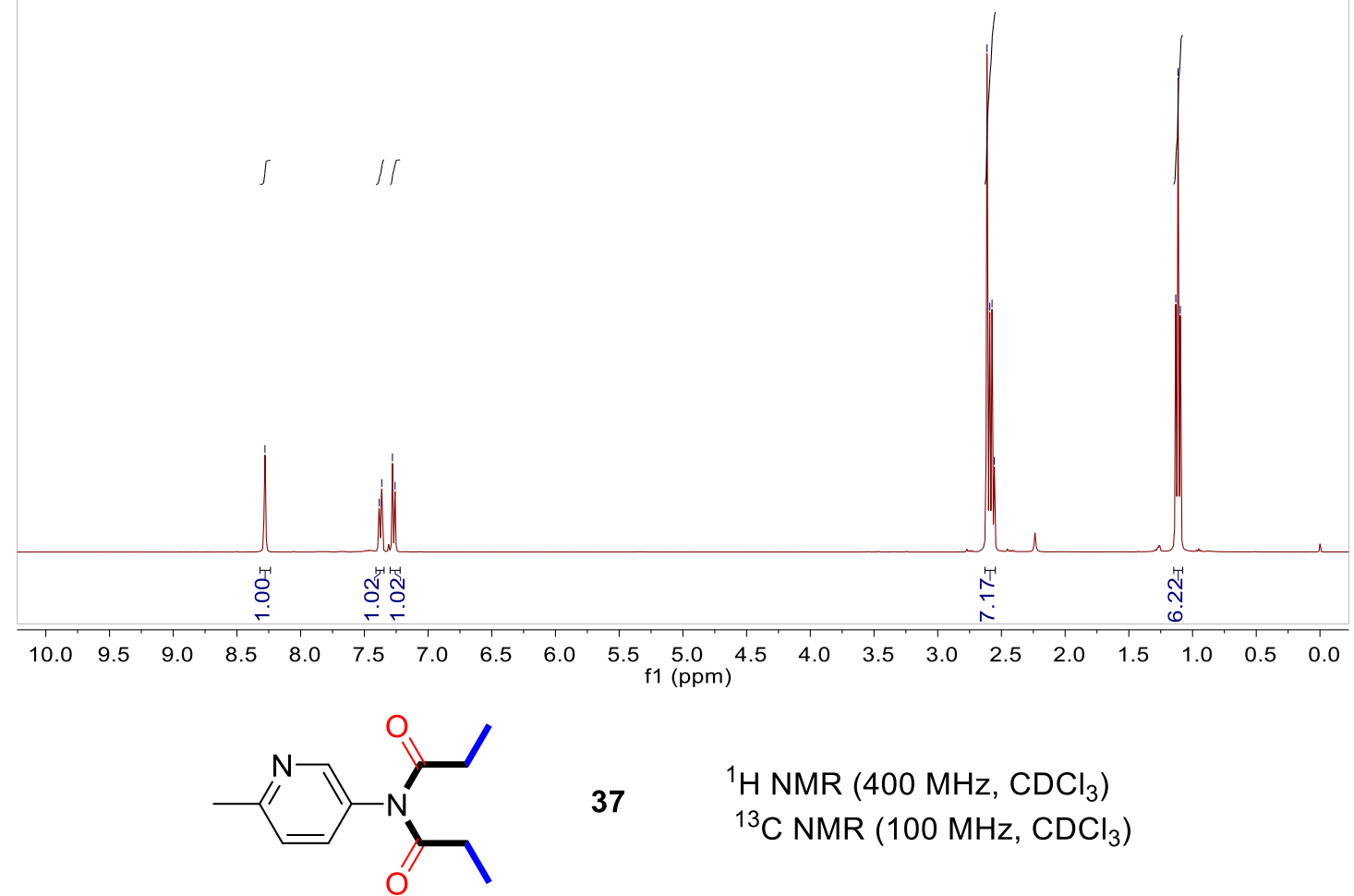

37

${ }^{1} \mathrm{H}$ NMR $\left(400 \mathrm{MHz}, \mathrm{CDCl}_{3}\right)$

${ }^{13} \mathrm{C}$ NMR $\left(100 \mathrm{MHz}, \mathrm{CDCl}_{3}\right)$

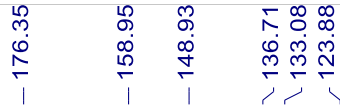

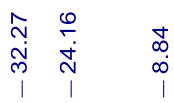

csk-3-18-5.11.fid

C13CPD CDCI3 \{D:INMR400ldn10604\} nmr-new 6

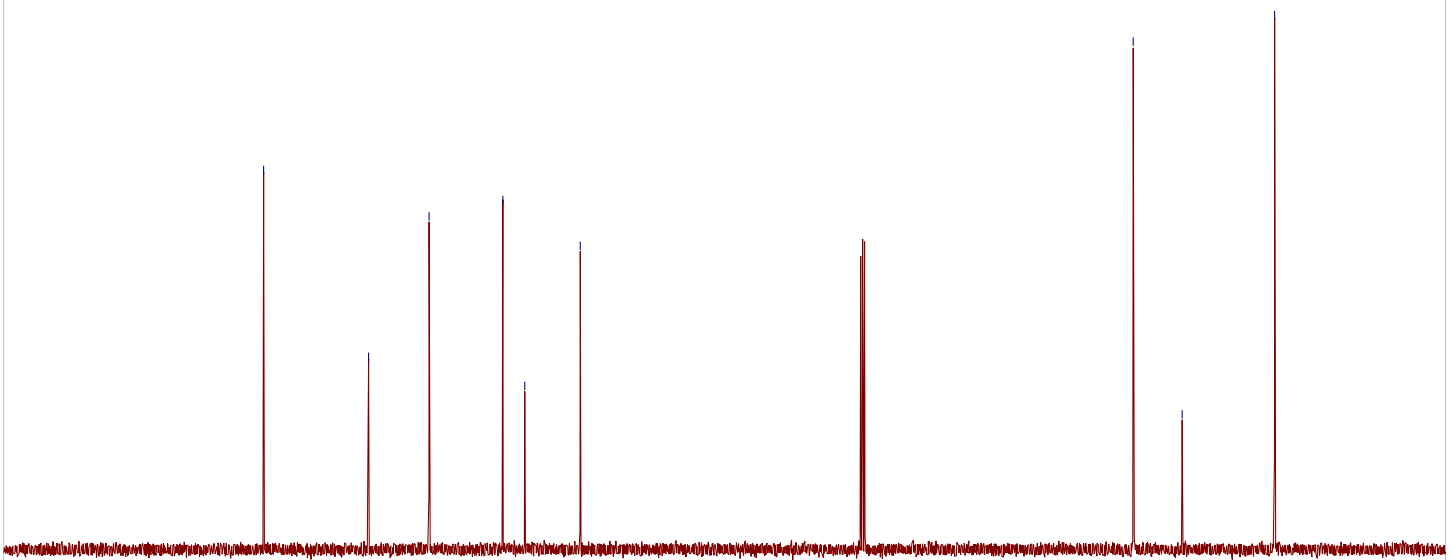

$\begin{array}{llllllllllllllllllllllll}210 & 200 & 190 & 180 & 170 & 160 & 150 & 140 & 130 & 120 & 110 & 100 & 90 & 80 & 70 & 60 & 50 & 40 & 30 & 20 & 10 & 0 & -10\end{array}$ f1 (ppm) 


舀

csk-3-18-4.10.fid

PROTON CDCI3 \{D:INMR400ldn10604\} nmr-new 5
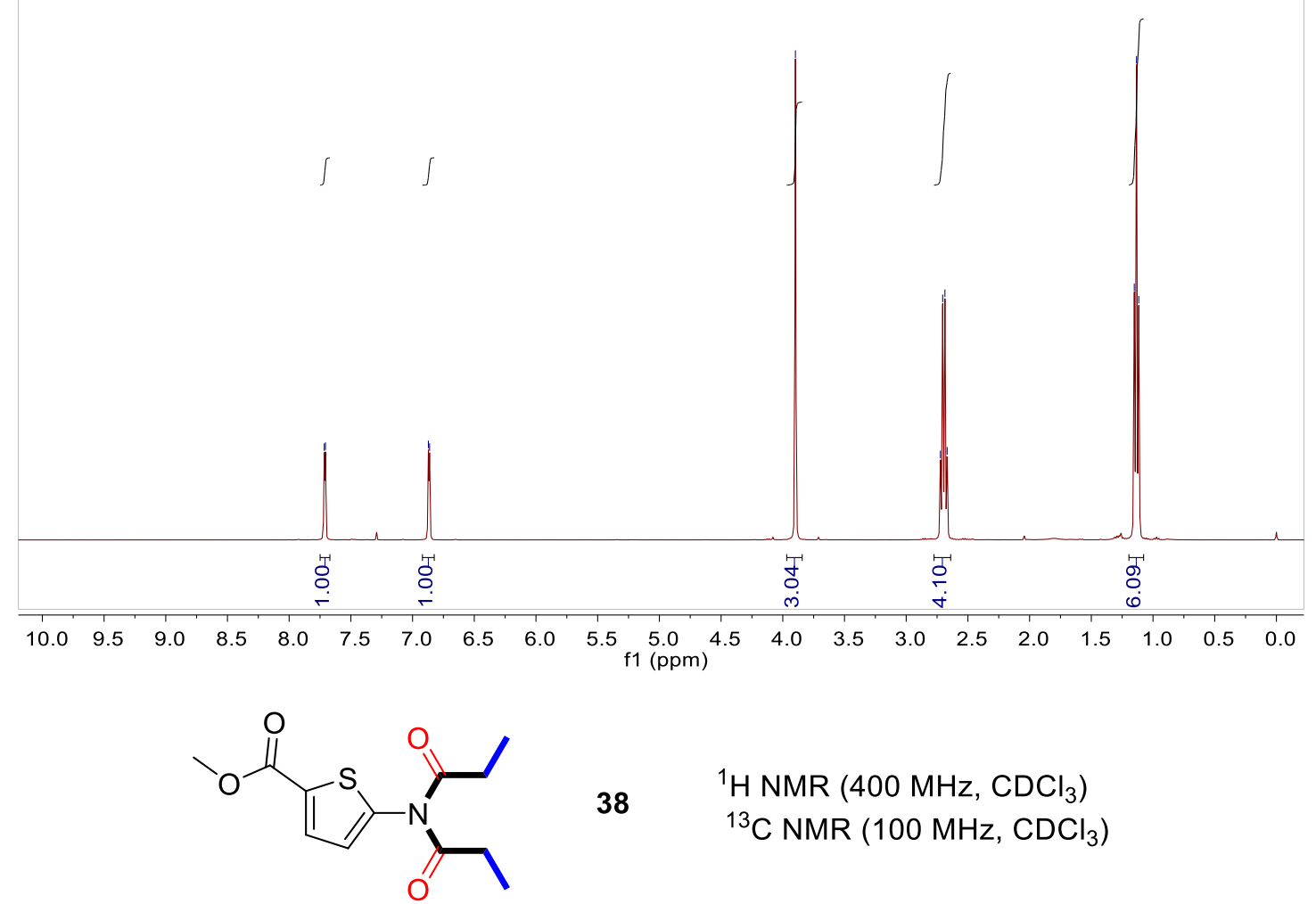

38

${ }^{1} \mathrm{H} \mathrm{NMR}\left(400 \mathrm{MHz}, \mathrm{CDCl}_{3}\right)$

${ }^{13} \mathrm{C}$ NMR $\left(100 \mathrm{MHz}, \mathrm{CDCl}_{3}\right)$

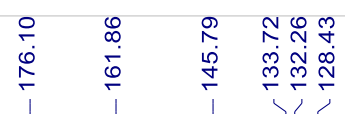

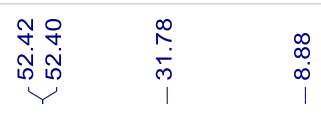

csk-3-18-4.11.fid

C13CPD CDCI3 \{D:INMR400ldnl0604\} nmr-new 5

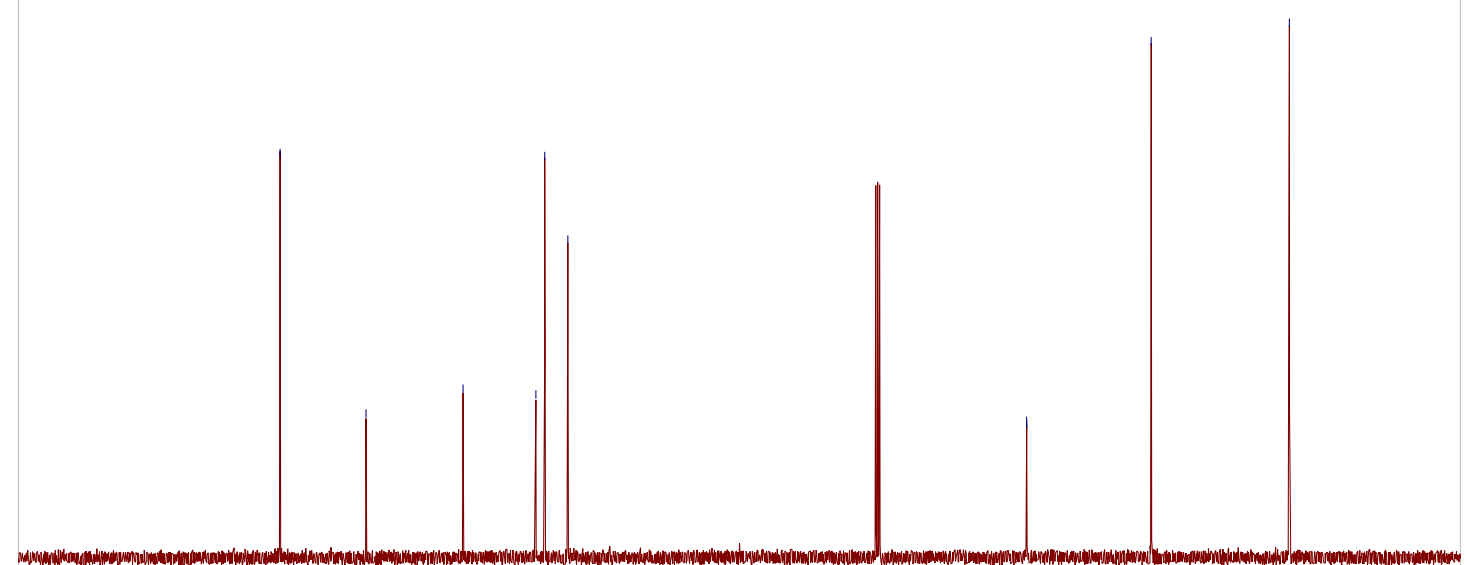

$\begin{array}{lllllllllllllllllllllll}210 & 200 & 190 & 180 & 170 & 160 & 150 & 140 & 130 & 120 & 110 & 100 & 90 & 80 & 70 & 60 & 50 & 40 & 30 & 20 & 10 & 0 & -10\end{array}$ f1 (ppm) 
csk-3-18-3.12.fid

PROTON CDCI3 \{D:INMR400Idnl0604\} nmr-new 47

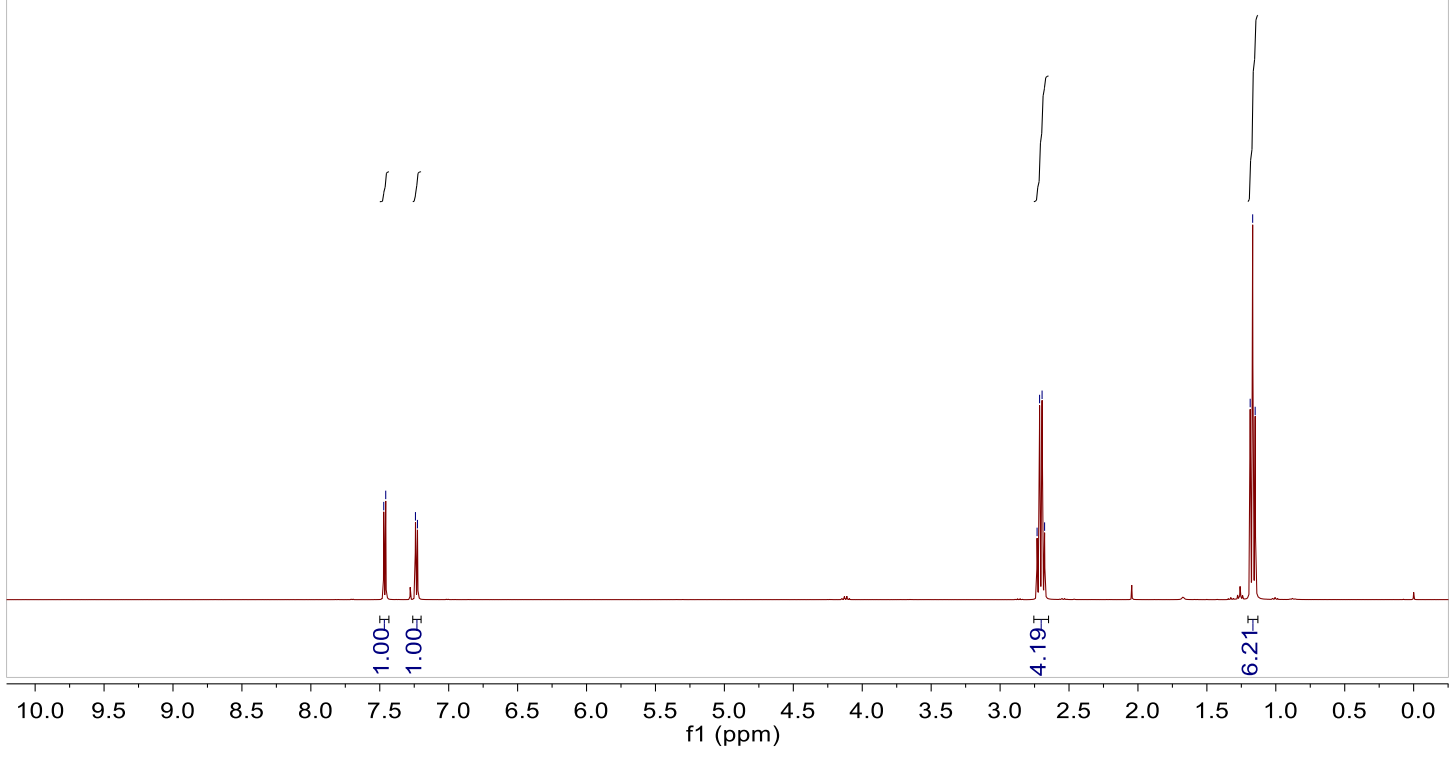<smiles>CCC(=O)N(C(=O)CC)c1sccc1C#N</smiles>

${ }^{13} \mathrm{C}$ NMR $\left(100 \mathrm{MHz}, \mathrm{CDCl}_{3}\right)$

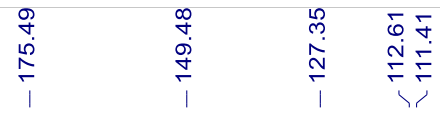

$\begin{array}{ll}m & 0 \\ \stackrel{n}{m} & \infty \\ 1 & \infty \\ 1 & 1\end{array}$

csk-3-18-3.13.fid

C13CPD CDCI3 \{D:INMR400ldn10604\} nmr-new 47

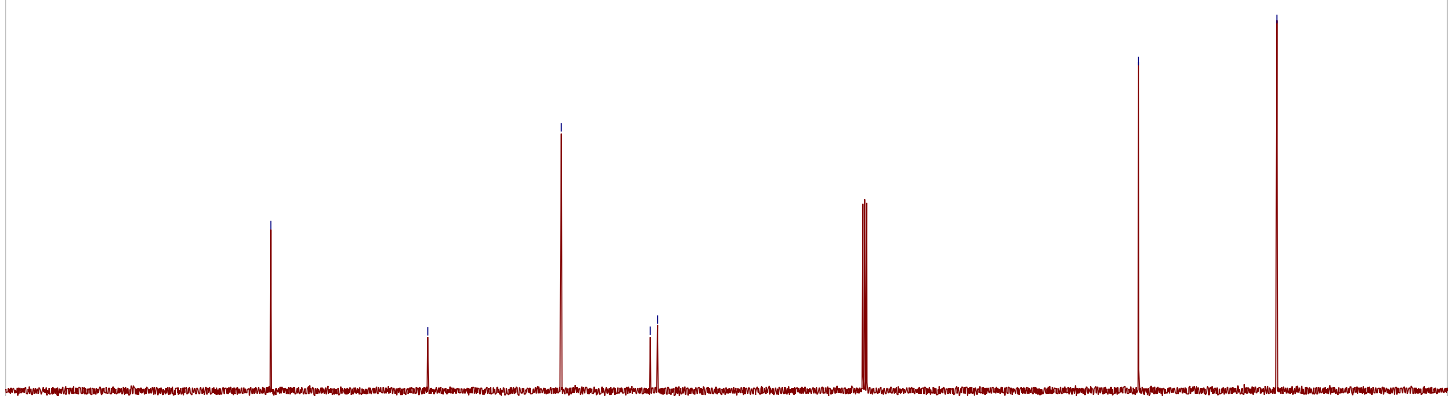

$\begin{array}{lllllllllllllllllllllll}210 & 200 & 190 & 180 & 170 & 160 & 150 & 140 & 130 & 120 & 110 & 100 & 90 & 80 & 70 & 60 & 50 & 40 & 30 & 20 & 10 & 0 & -10\end{array}$ 
csk-3-5-17.10.fid

PROTON CDCI3 \{D:INMR400Idnl0604\} nmr-new 33
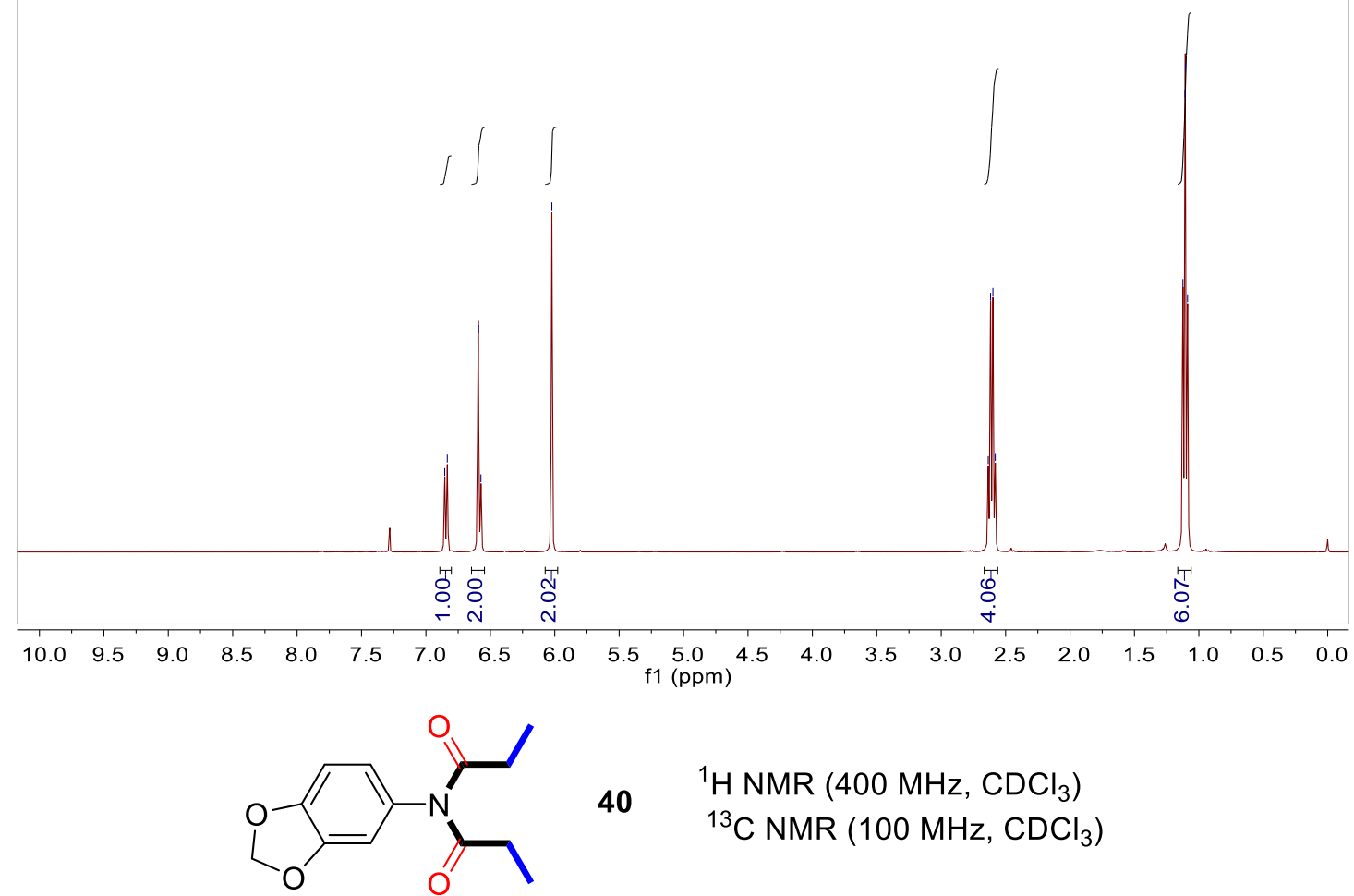

40

${ }^{1} \mathrm{H}$ NMR $\left(400 \mathrm{MHz}, \mathrm{CDCl}_{3}\right)$

${ }^{13} \mathrm{C}$ NMR (100 MHz, $\mathrm{CDCl}_{3}$ )

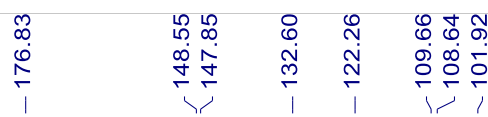

$\begin{array}{ll}\text { के } & \hat{n} \\ \text { ले } & \infty \\ 1 & 1\end{array}$

sk-3-5-17.11.fid

C13CPD CDCI3 \{D:INMR400ldnl0604\} nmr-new 33

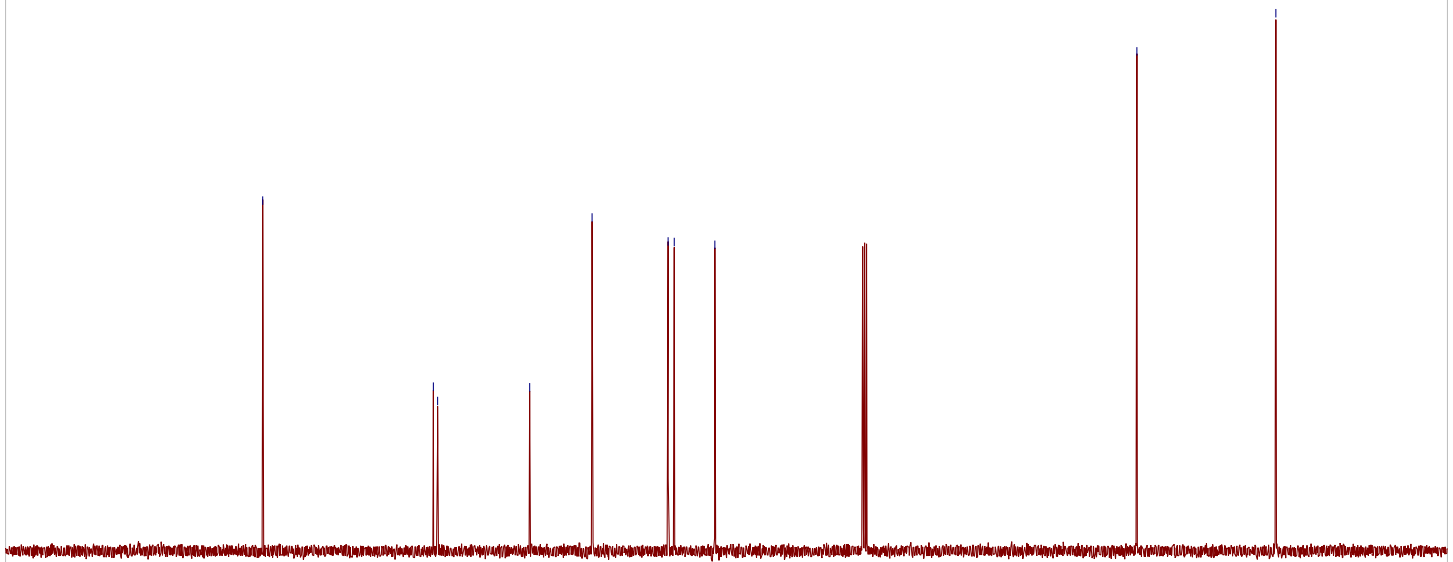

$\begin{array}{llllllllllllllllllllllll}210 & 200 & 190 & 180 & 170 & 160 & 150 & 140 & 130 & 120 & 110 & 100 & 90 & 80 & 70 & 60 & 50 & 40 & 30 & 20 & 10 & 0 & -10\end{array}$ f1 (ppm) 
csk-3-12-8.10.fid

PROTON CDCI3 \{D:INMR400IDNL0604\} nmr-new 35
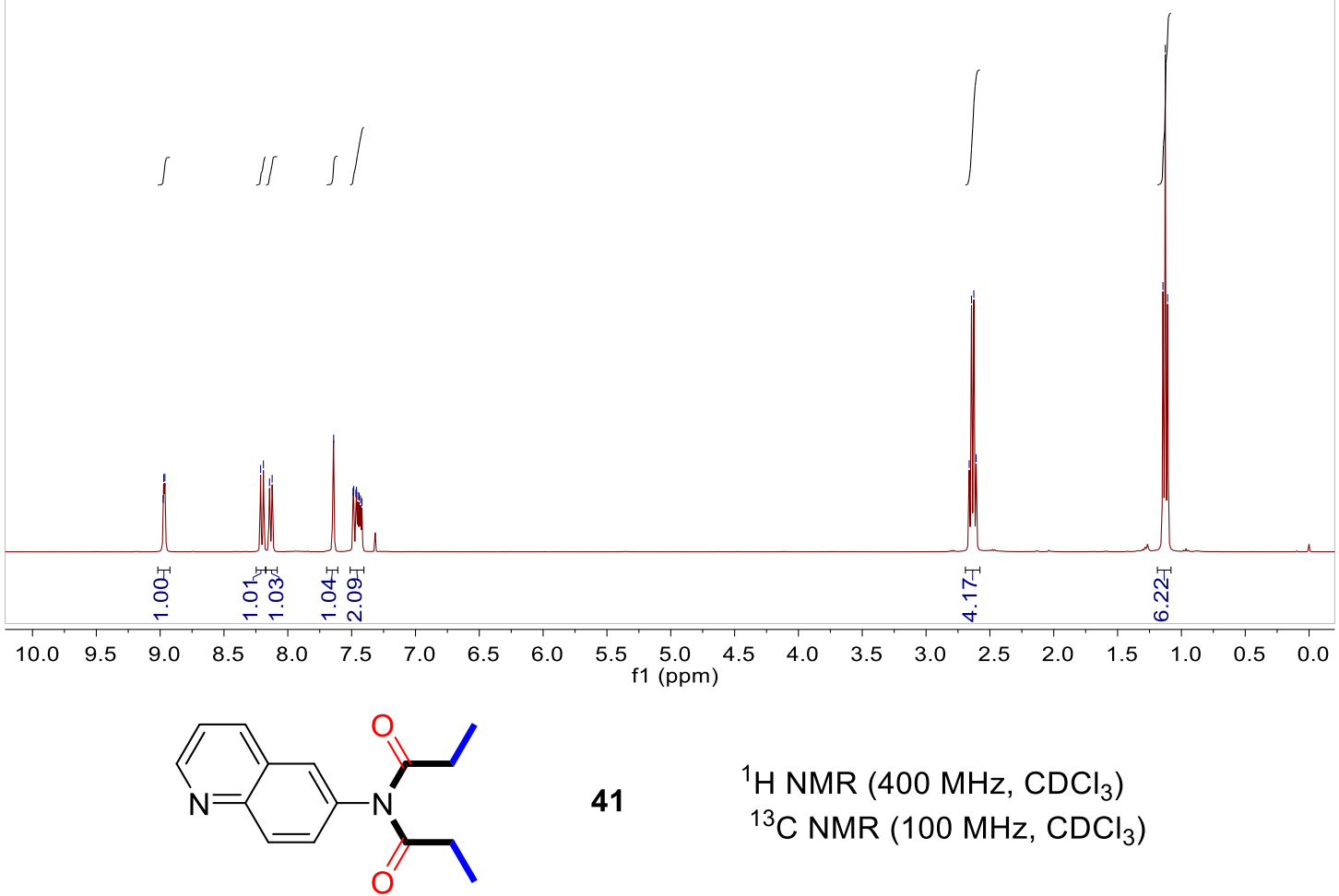

41

${ }^{1} \mathrm{H}$ NMR (400 MHz, $\left.\mathrm{CDCl}_{3}\right)$

${ }^{13} \mathrm{C}$ NMR $\left(100 \mathrm{MHz}, \mathrm{CDCl}_{3}\right)$

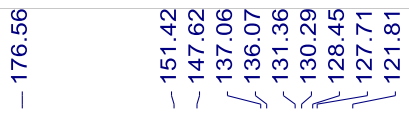

csk-3-12-8.11. fid

C13CPD CDCI3 \{D:INMR400IDNL0604\} nmr-new 35

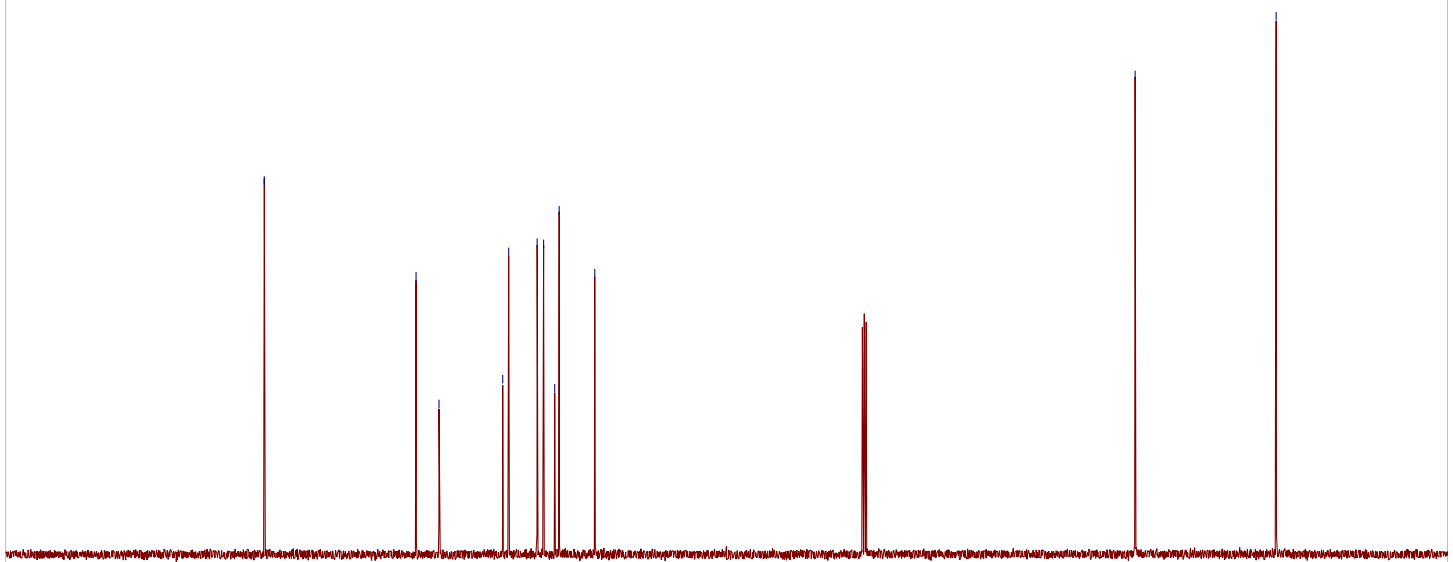

$\begin{array}{lllllllllllllllllllllll}210 & 200 & 190 & 180 & 170 & 160 & 150 & 140 & 130 & 120 & 110 & 100 & 90 & 80 & 70 & 60 & 50 & 40 & 30 & 20 & 10 & 0 & -10\end{array}$ 
csk-3-18-6.10.fid

PROTON CDCI3 \{D:INMR400Idnl0604\} nmr-new 7
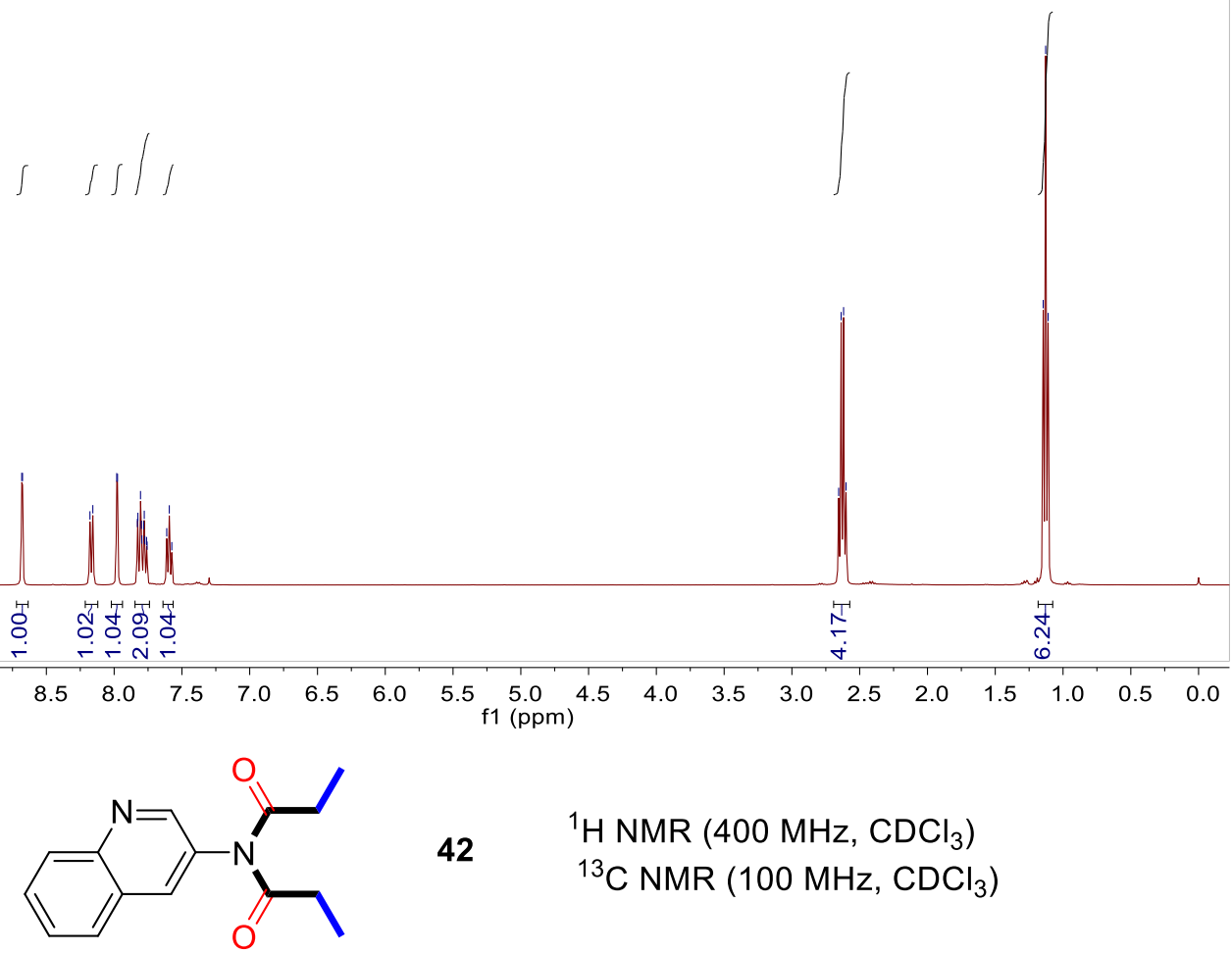

42

${ }^{1} \mathrm{H}$ NMR (400 MHz, $\left.\mathrm{CDCl}_{3}\right)$

${ }^{13} \mathrm{C}$ NMR $\left(100 \mathrm{MHz}, \mathrm{CDCl}_{3}\right)$

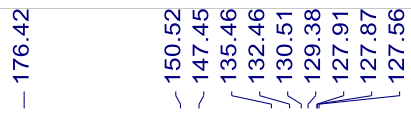

\begin{tabular}{ll}
\multirow{+}{*}{} & $\infty$ \\
& $\infty$ \\
1 & $\infty$ \\
1 & 1
\end{tabular}

csk-3-18-6.11.fid

C13CPD CDCI3 \{D:INMR400Idnl0604\} nmr-new 7

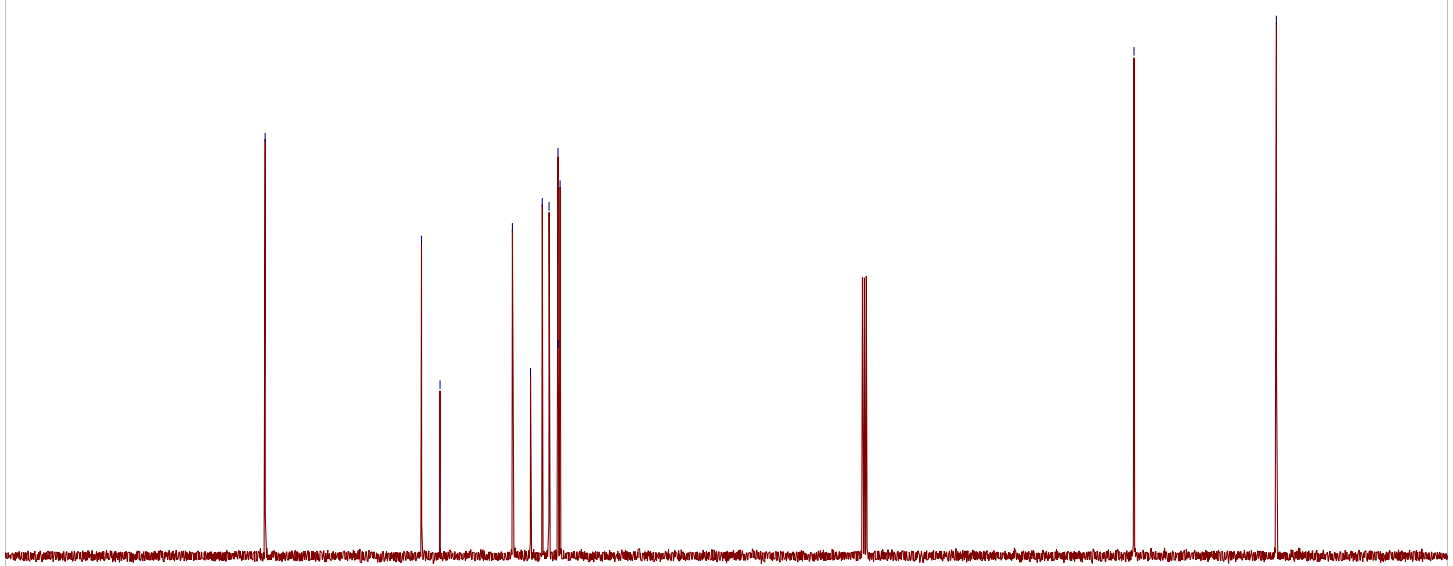

$\begin{array}{llllllllllllllllllllllll}210 & 200 & 190 & 180 & 170 & 160 & 150 & 140 & 130 & 120 & 110 & 100 & 90 & 80 & 70 & 60 & 50 & 40 & 30 & 20 & 10 & 0 & -10\end{array}$ 
csk-3-18-2.10.fid

PROTON CDCI3 \{D:INMR400Idnl0604\} nmr-new 3
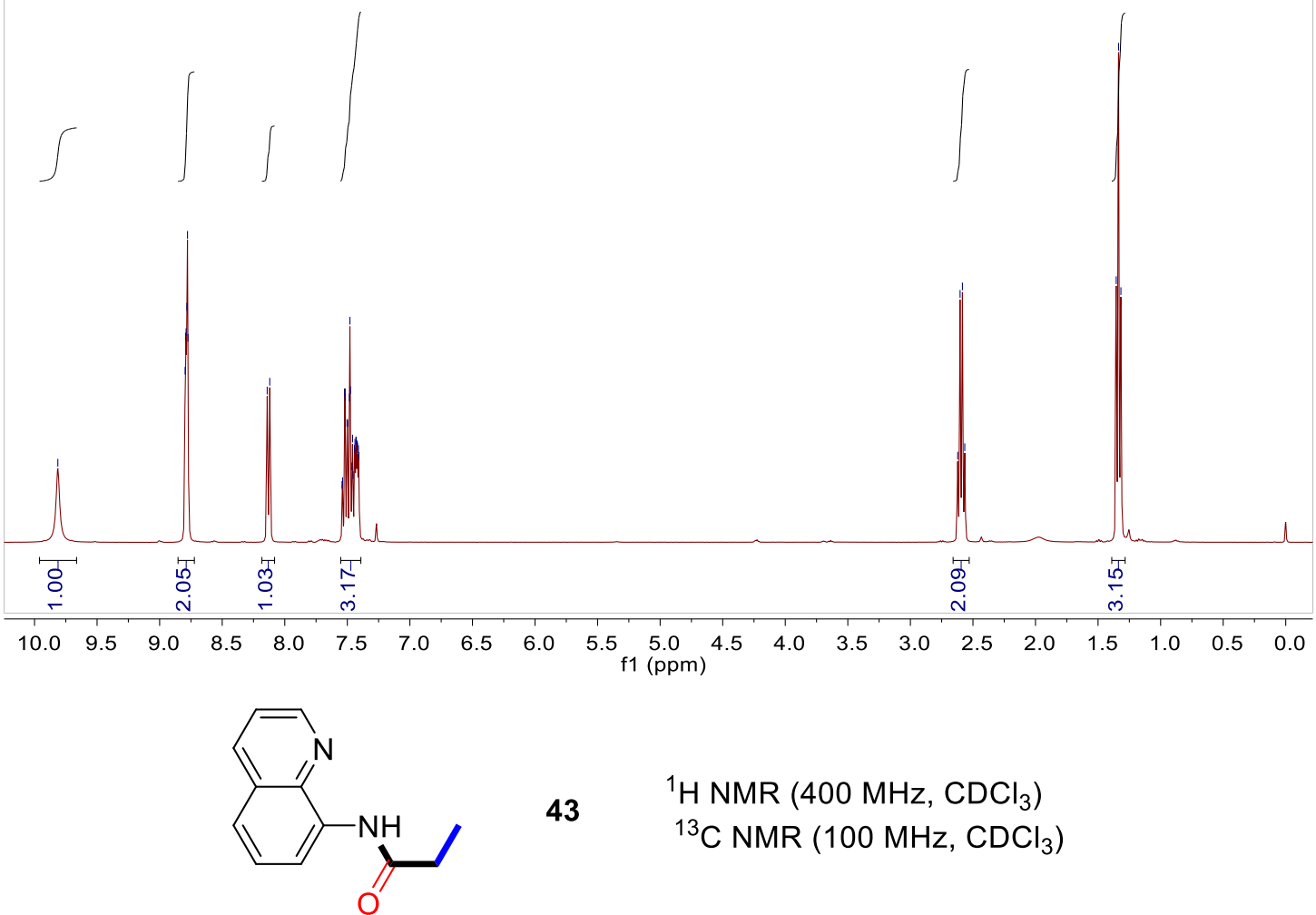

43

${ }^{1} \mathrm{H}$ NMR $\left(400 \mathrm{MHz}, \mathrm{CDCl}_{3}\right)$

${ }^{13} \mathrm{C}$ NMR $\left(100 \mathrm{MHz}, \mathrm{CDCl}_{3}\right)$

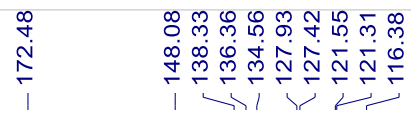

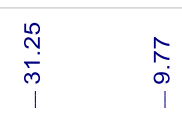

csk-3-18-2.11.fid

C13CPD CDCI3 \{D:INMR400ldn10604\} nmr-new 3

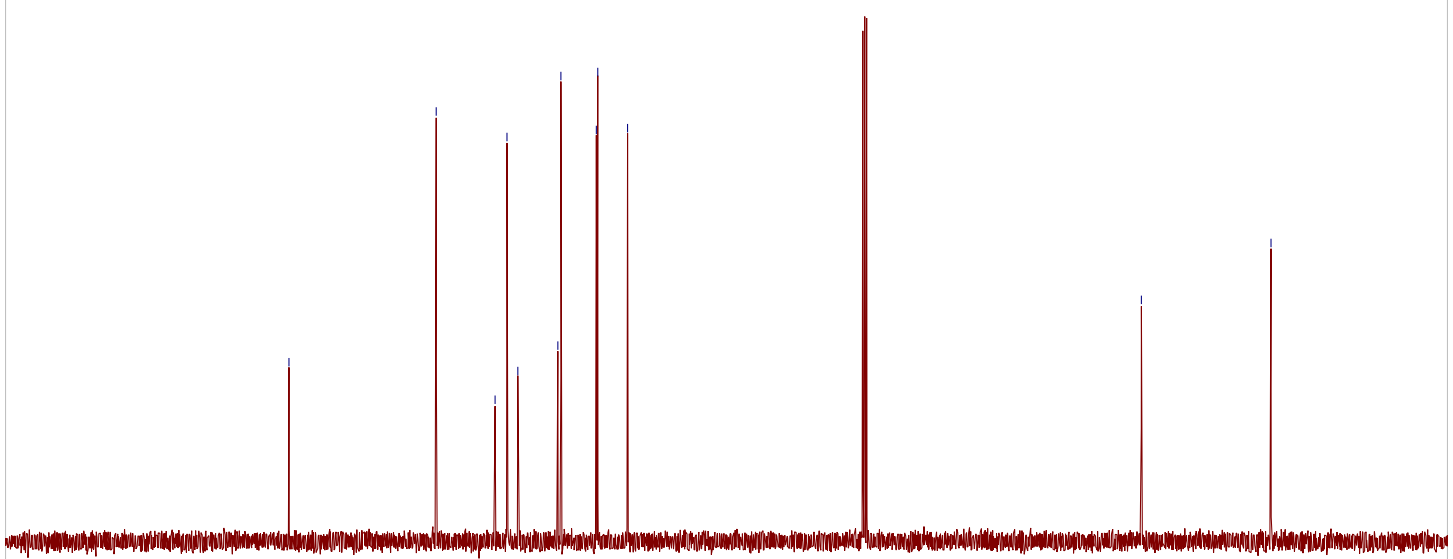

$\begin{array}{lllllllllllllllllllllll}210 & 200 & 190 & 180 & 170 & 160 & 150 & 140 & 130 & 120 & 110 & 100 & 90 & 80 & 70 & 60 & 50 & 40 & 30 & 20 & 10 & 0 & -10\end{array}$ f1 (ppm) 
csk-3-12-3-s.10.fid

PROTON CDCI3 \{D:INMR400ldnl0604\} nmr-new 4

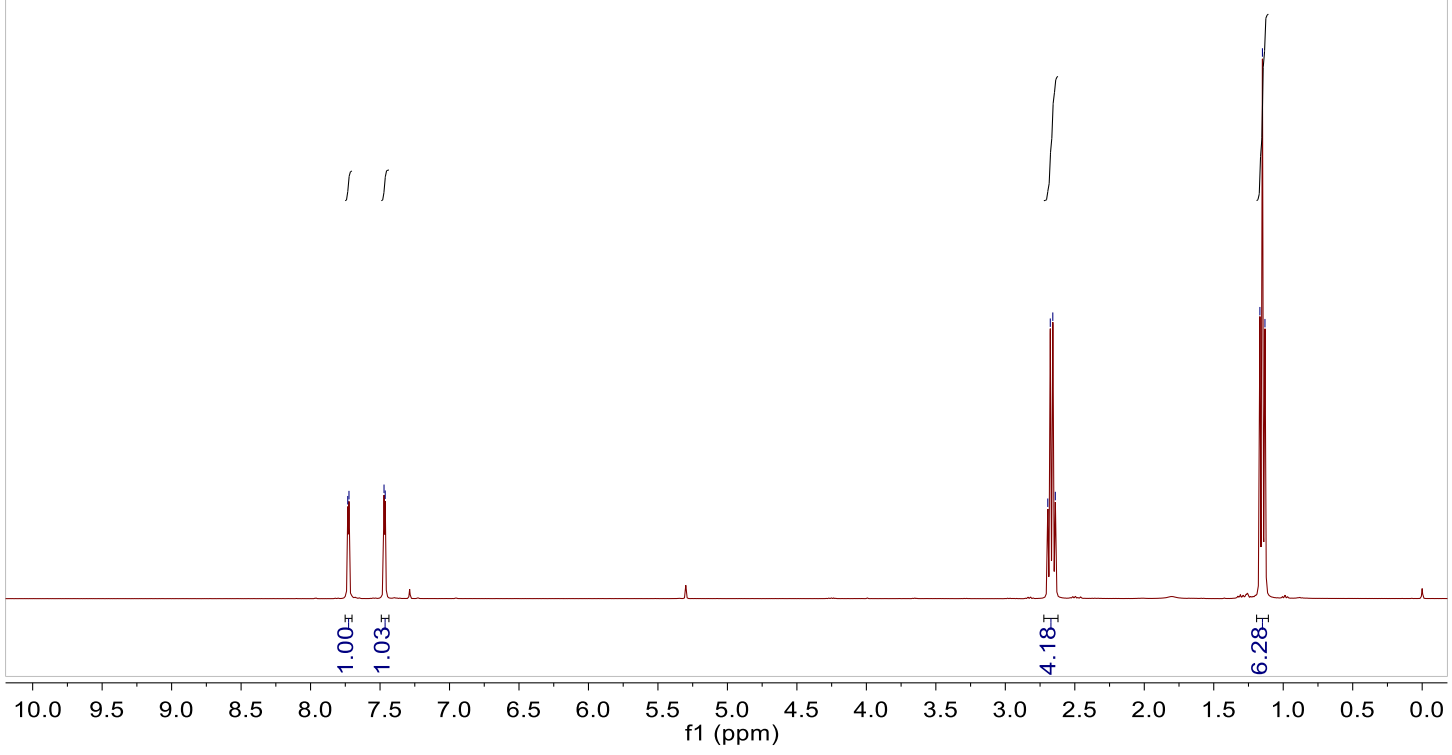<smiles>CCC(=O)N(C(=O)CC)c1nccs1</smiles>

${ }^{13} \mathrm{C}$ NMR $\left(100 \mathrm{MHz}, \mathrm{CDCl}_{3}\right)$

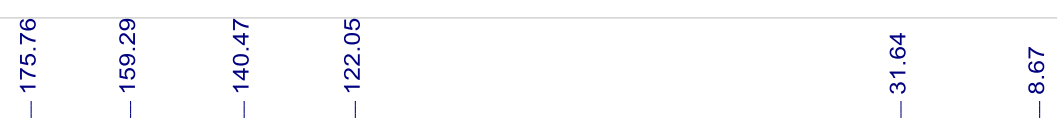

csk-3-12-3-s.11.fid

C13CPD CDCI3 \{D:INMR400ldn10604\} nmr-new 4

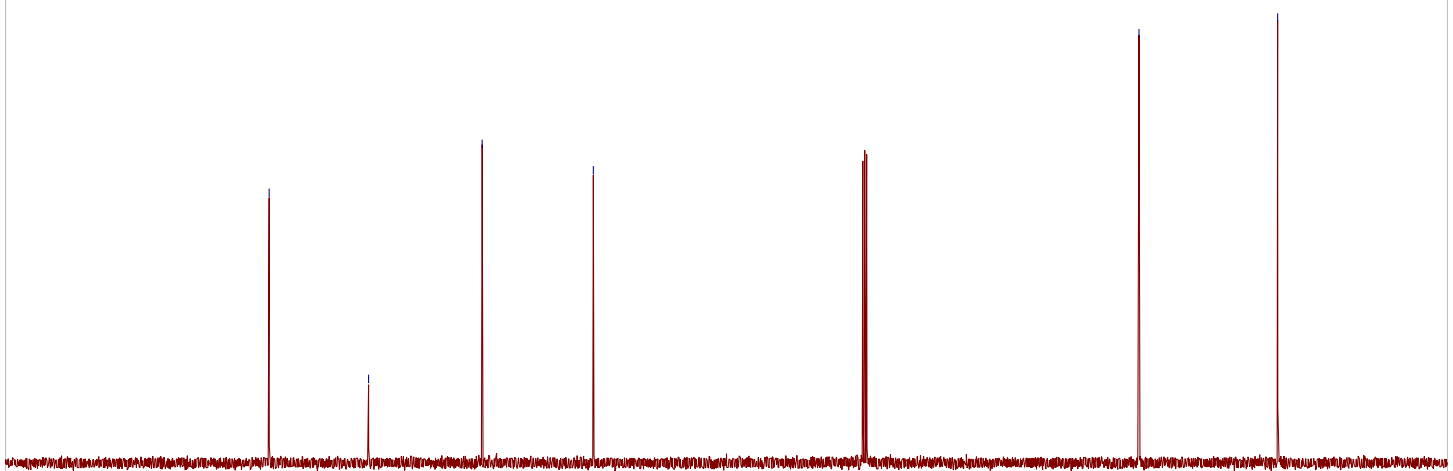

$\begin{array}{lllllllllllll}210 & 200 & 190 & 180 & 170 & 160 & 150 & 140 & 130 & 120 & 110 & 100 & 90\end{array}$ 

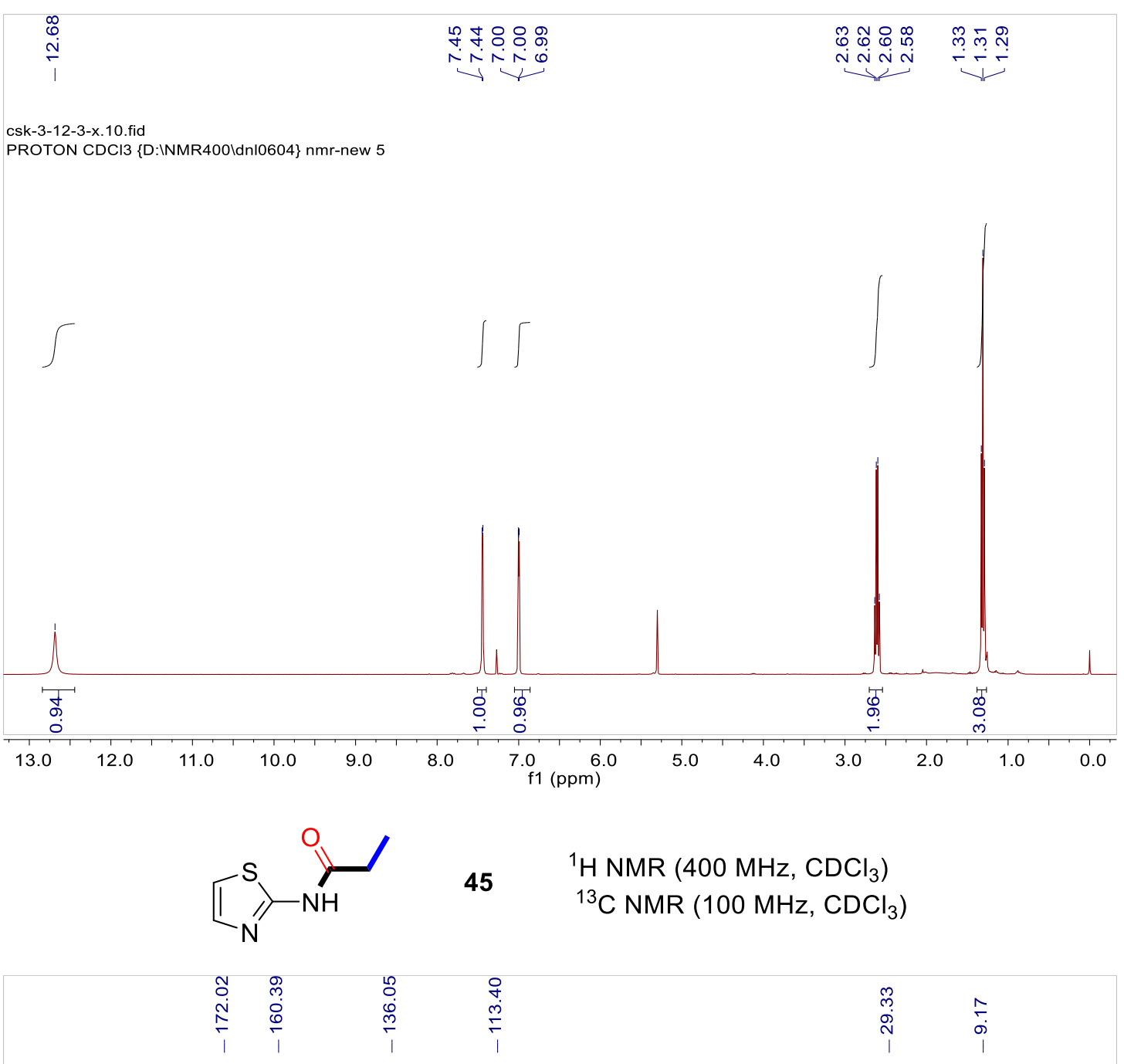

csk-3-12-3-x.11.fid

C13CPD CDCI3 \{D:INMR400ldnl0604\} nmr-new 5

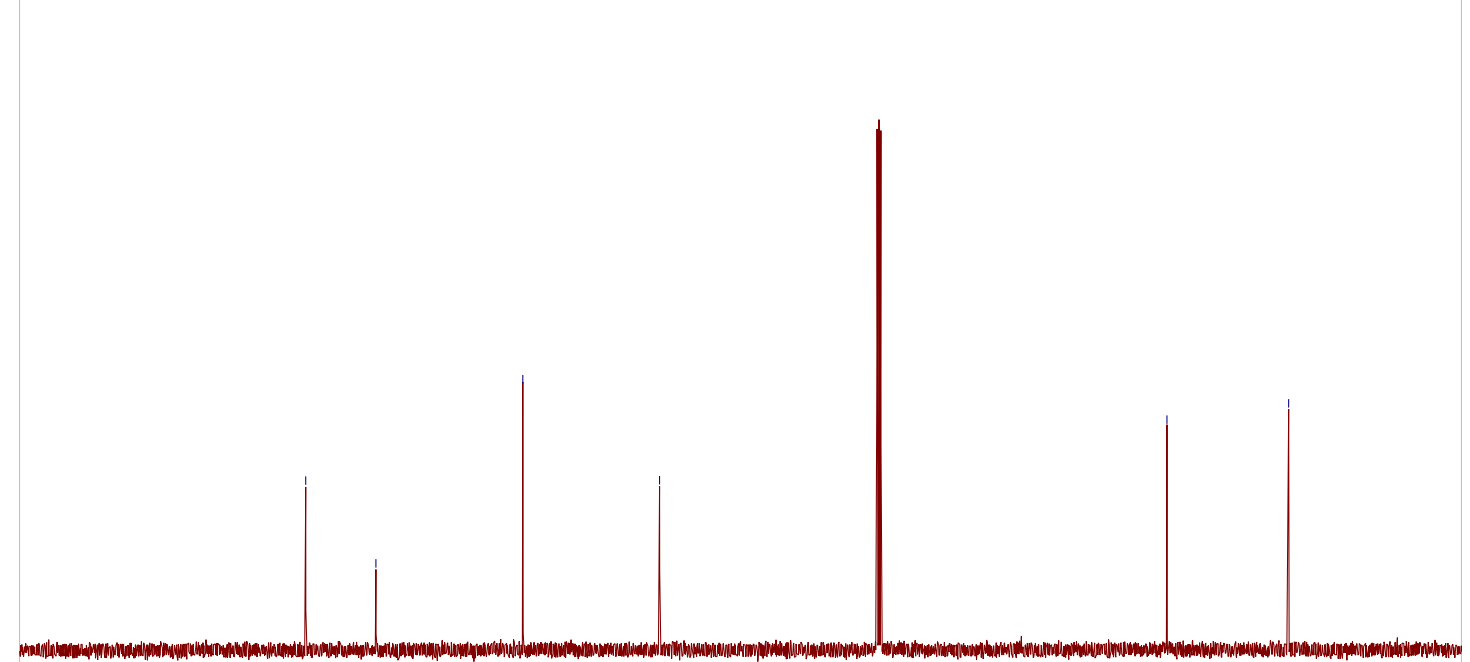

$\begin{array}{lllllllllllllllllllllllllll}210 & 200 & 190 & 180 & 170 & 160 & 150 & 140 & 130 & 120 & 110 & 100 & 90 & 80 & 70 & 60 & 50 & 40 & 30 & 20 & 10 & 0 & -10\end{array}$ 
csk-3-12-1-s.10.fid

PROTON CDCI3 \{D:INMR400ldnl0604\} nmr-new 1

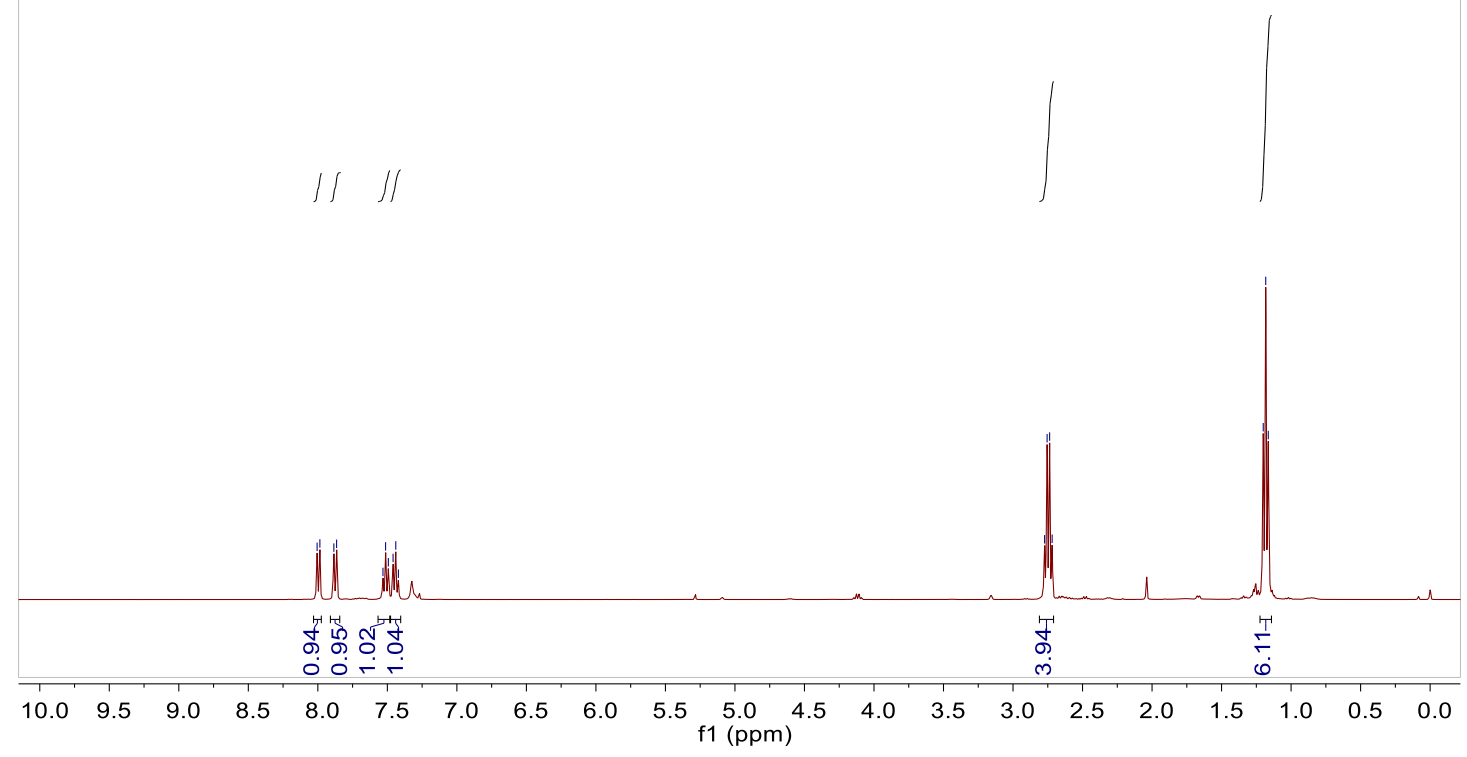<smiles>CCC(=O)N(C(=O)CC)c1nc2ccccc2s1</smiles>

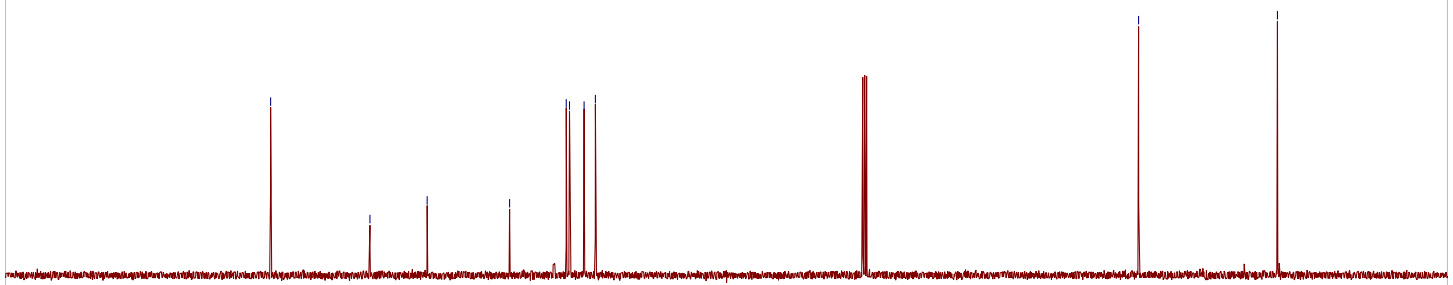

$\begin{array}{llllllllllll}210 & 200 & 190 & 180 & 170 & 160 & 150 & 140 & 130 & 120 & 110 & 100\end{array}$ 


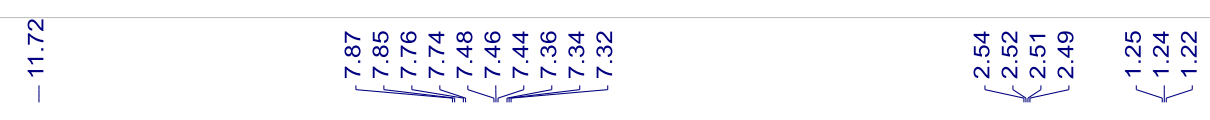

csk-3-12-1-x.10.fid

PROTON CDCI3 \{D:INMR400ldnl0604\} nmr-new 2
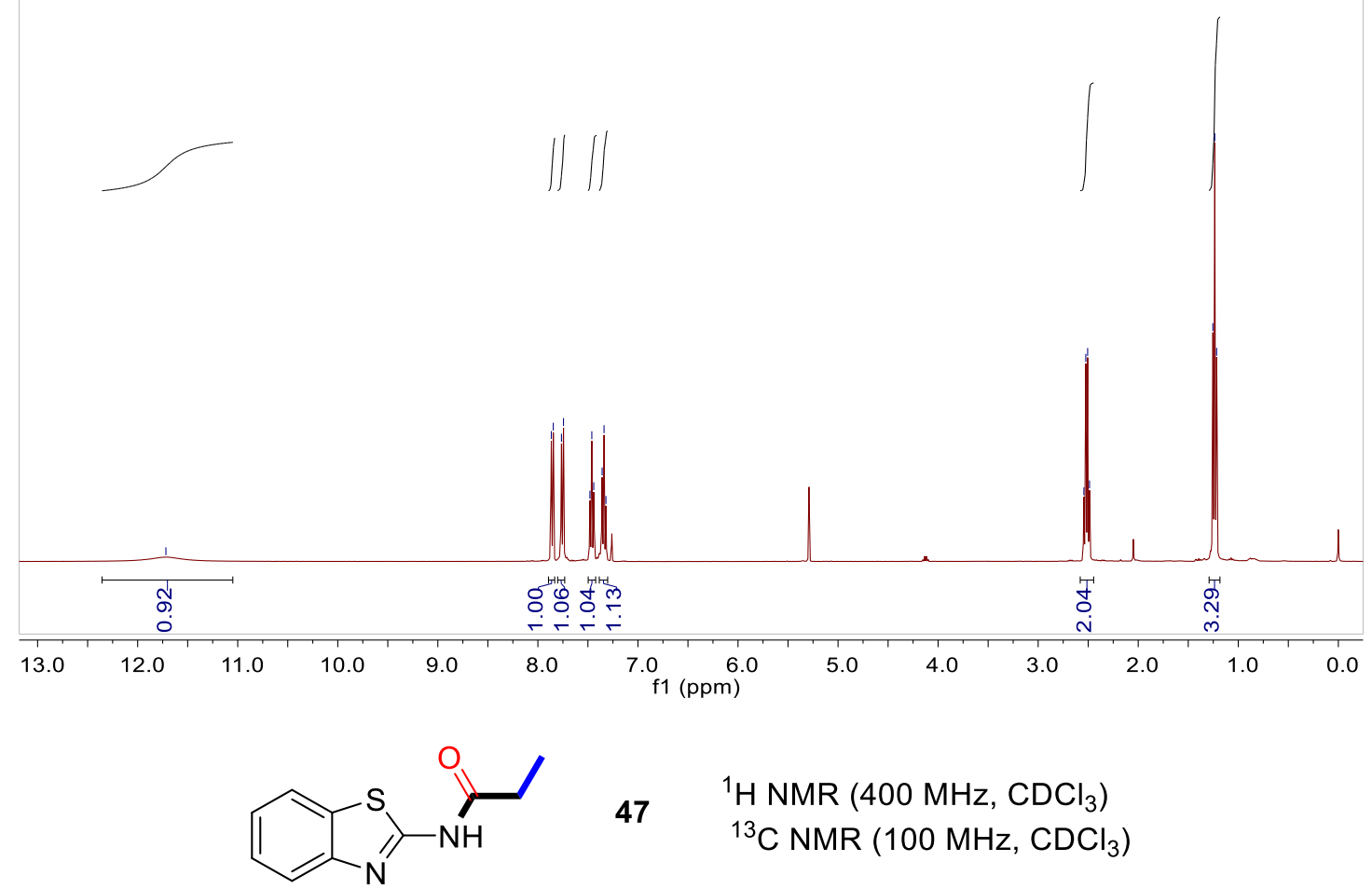

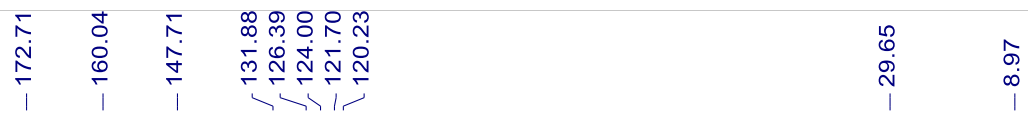

csk-3-12-1-x.11.fid

C13CPD CDCI3 \{D:INMR400ldn10604\} nmr-new 2

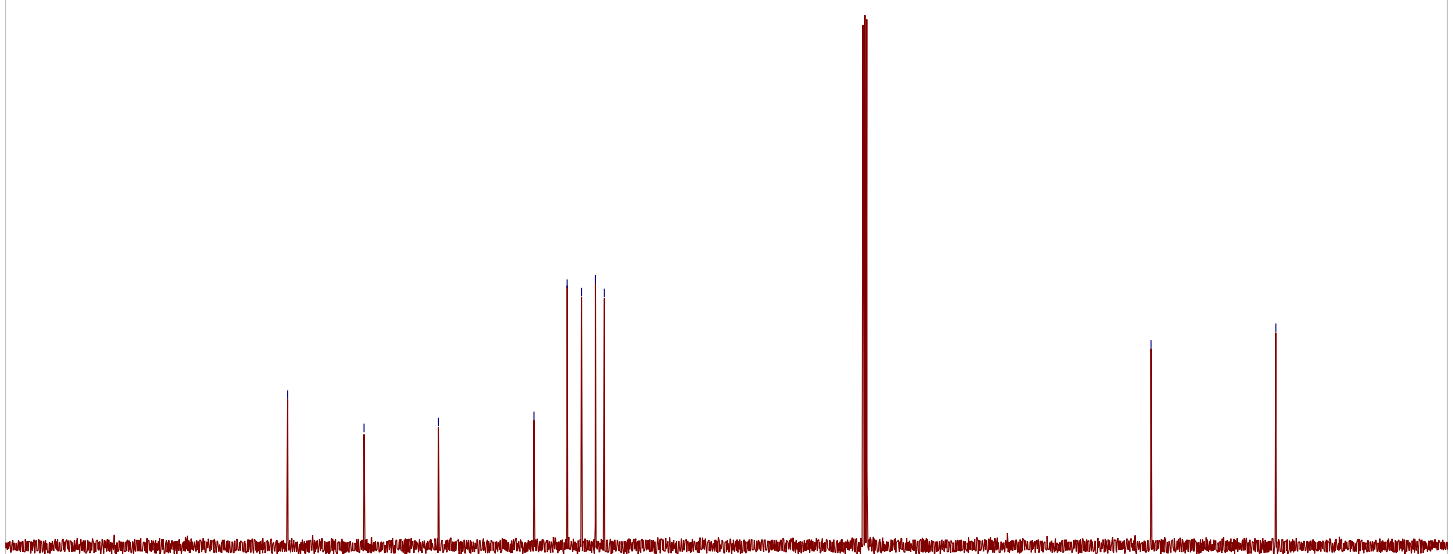

$\begin{array}{llllllllllllllllllllllll}210 & 200 & 190 & 180 & 170 & 160 & 150 & 140 & 130 & 120 & 110 & 100 & 90 & 80 & 70 & 60 & 50 & 40 & 30 & 20 & 10 & 0 & -10\end{array}$ 
csk-3-23-1.10.fid

PROTON CDCI3 \{D:INMR400IDNL0604\} nmr-new 60
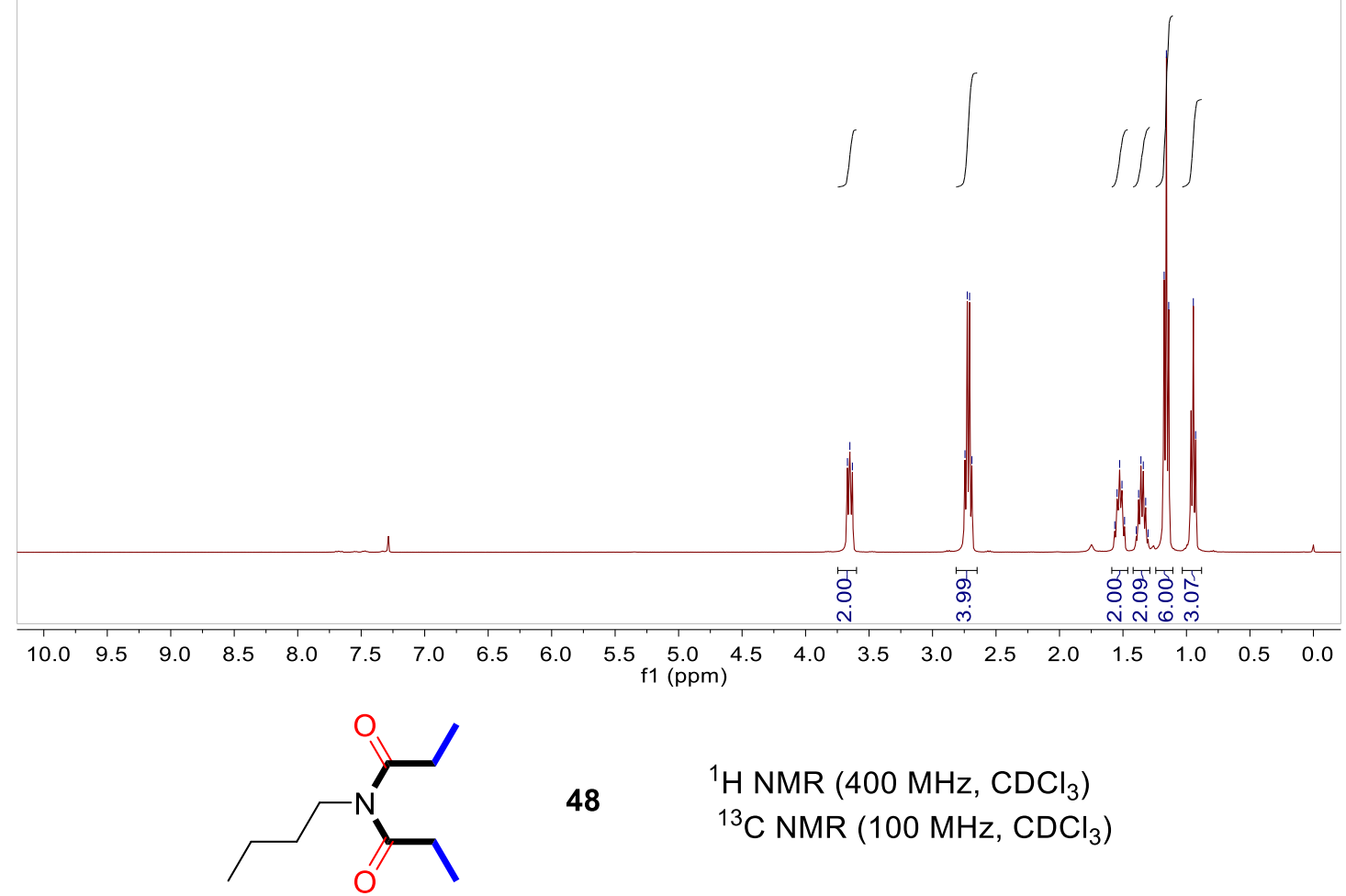

48

${ }^{1} \mathrm{H}$ NMR $\left(400 \mathrm{MHz}, \mathrm{CDCl}_{3}\right)$

${ }^{13} \mathrm{C}$ NMR $\left(100 \mathrm{MHz}, \mathrm{CDCl}_{3}\right)$

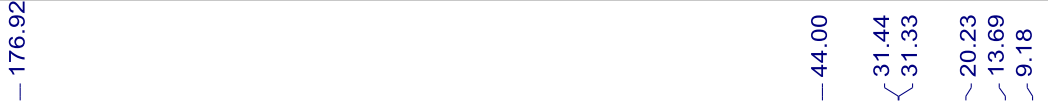

csk-3-23-1.11.fid

C13CPD CDCI3 \{D:INMR400IDNL0604\} nmr-new 60

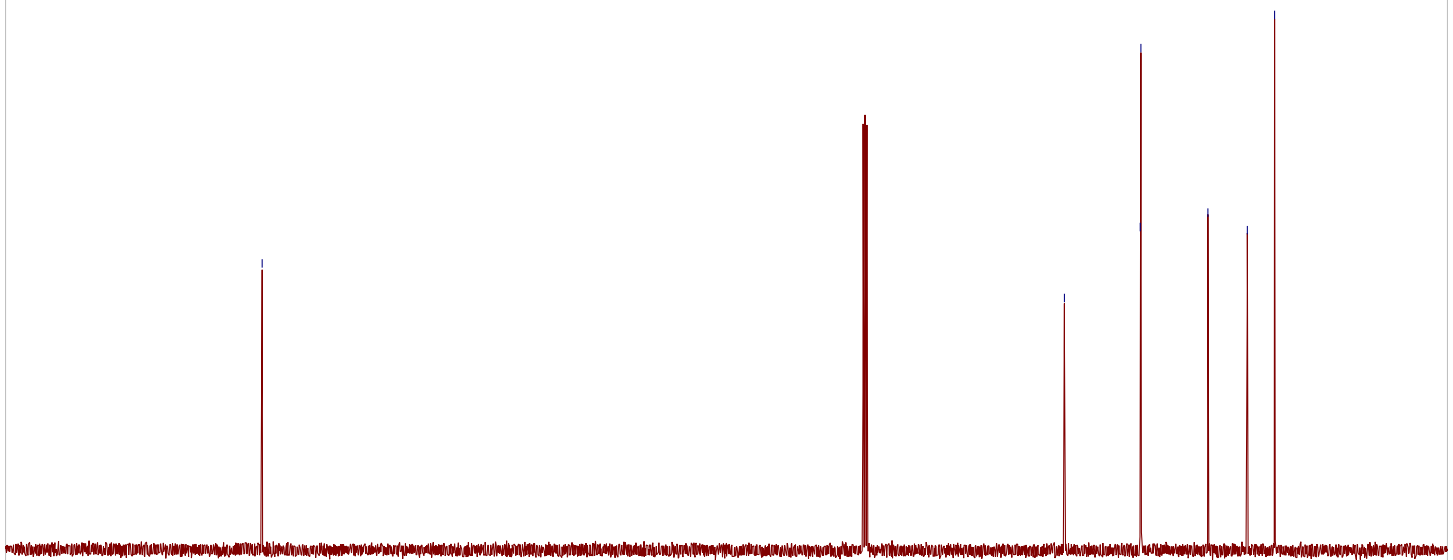

$\begin{array}{lllllllllllllllllllllll}210 & 200 & 190 & 180 & 170 & 160 & 150 & 140 & 130 & 120 & 110 & 100 & 90 & 80 & 70 & 60 & 50 & 40 & 30 & 20 & 10 & 0 & -10\end{array}$ 
csk-3-23-4.10.fid

PROTON CDCI3 \{D:INMR400IDNL0604\} nmr-new 23

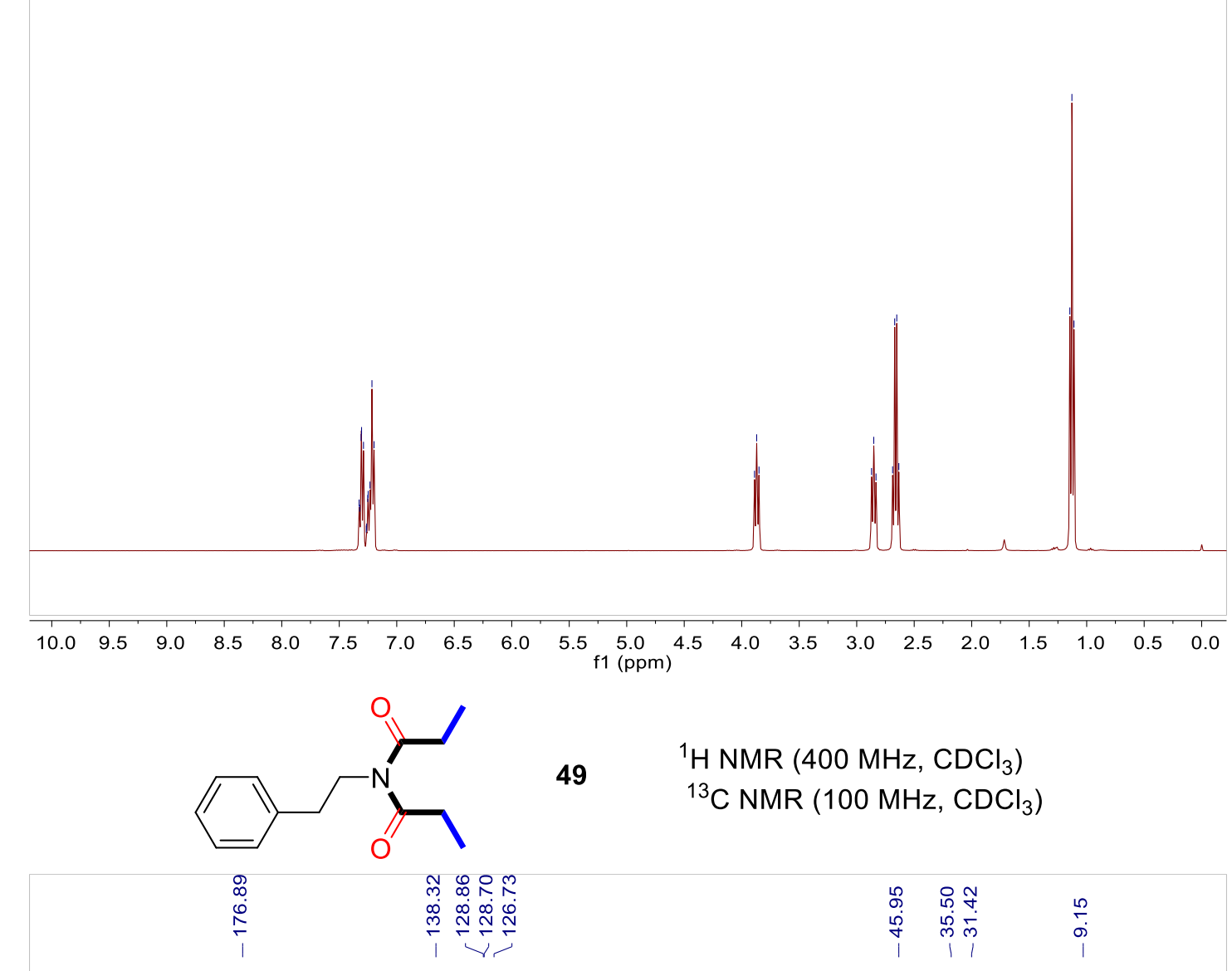

csk-3-23-4.11.fid

C13CPD CDCI3 \{D:INMR400IDNL0604\} nmr-new 23

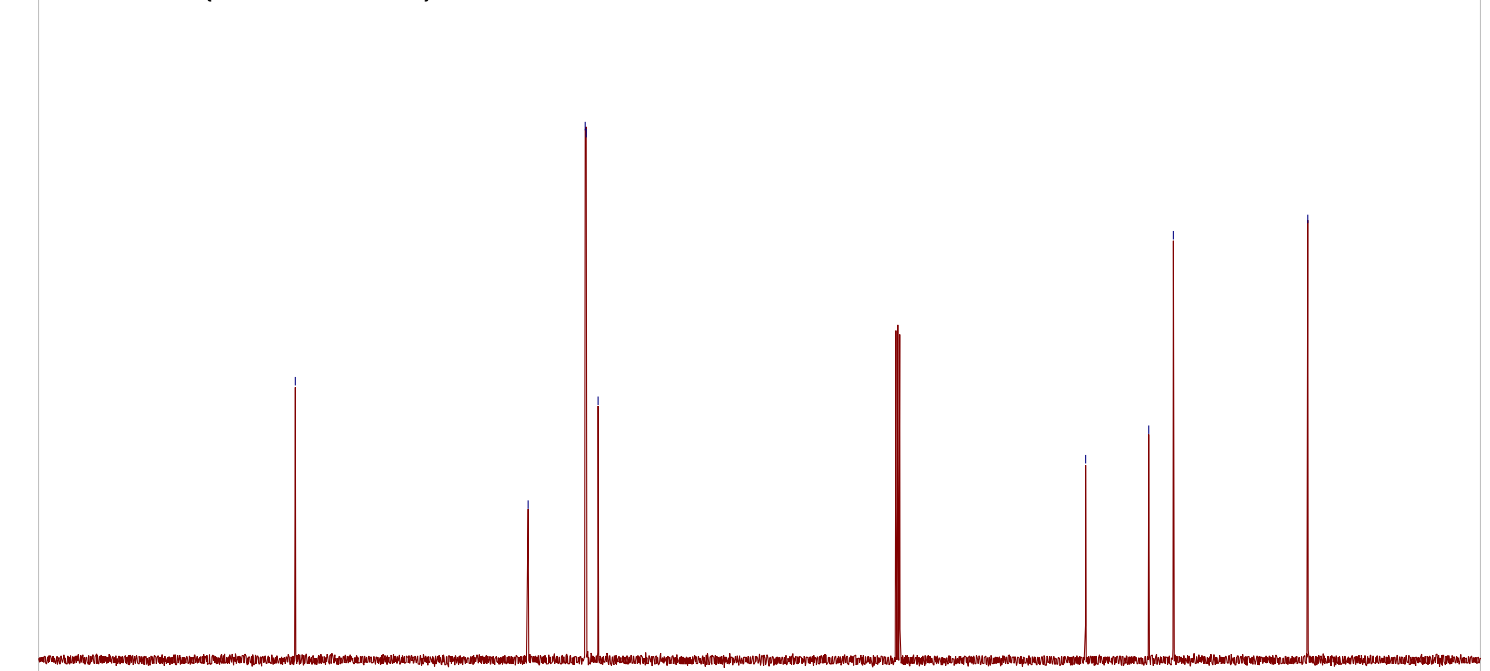

$\begin{array}{lllllllllllllllllllllllllll}210 & 200 & 190 & 180 & 170 & 160 & 150 & 140 & 130 & 120 & 110 & 100 & 90 & 80 & 70 & 60 & 50 & 40 & 30 & 20 & 10 & 0 & -10\end{array}$ 
csk-3-23-3.10.fid

PROTON CDCI3 \{D:INMR400IDNL0604\} nmr-new 22
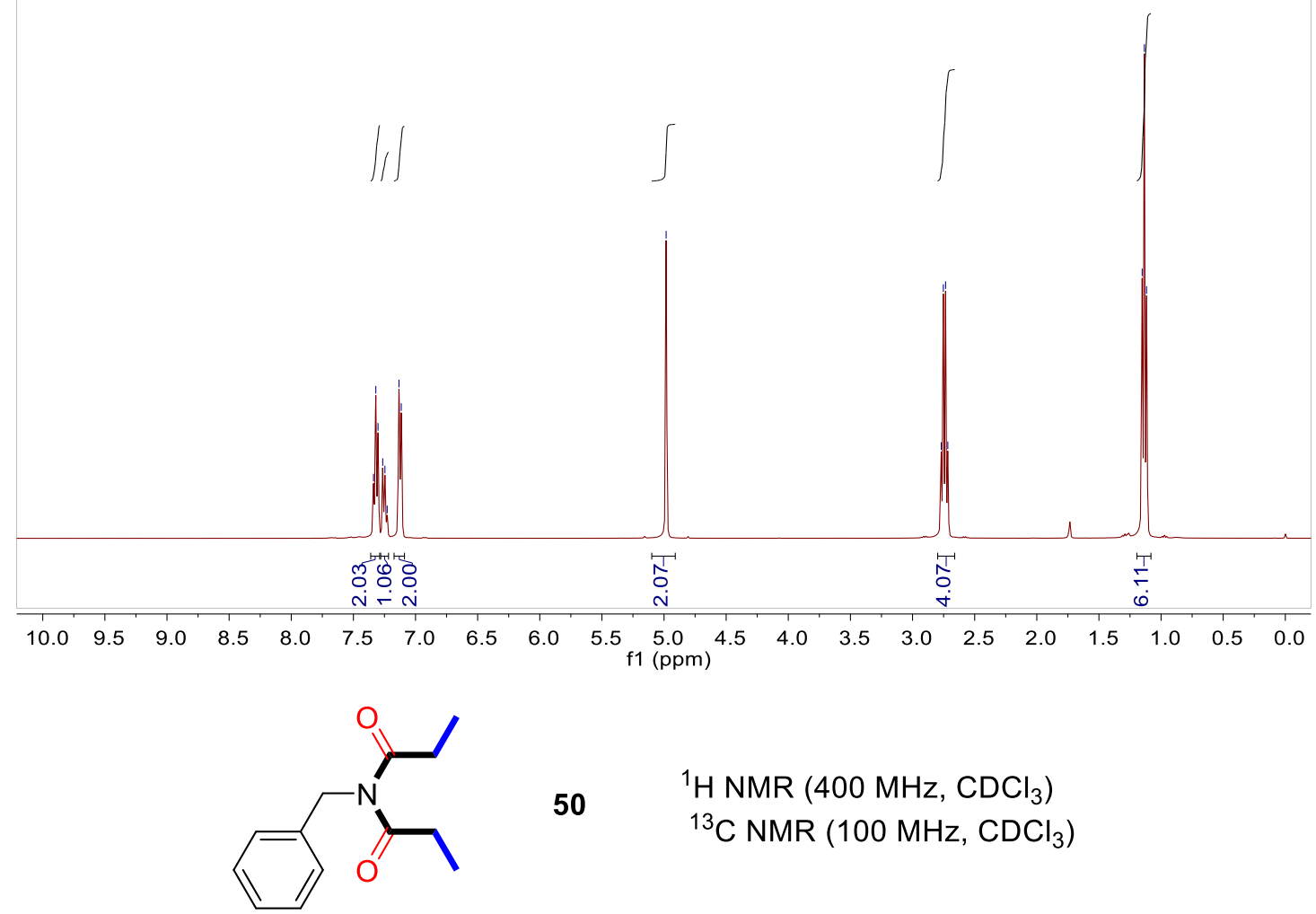

50

${ }^{1} \mathrm{H}$ NMR $\left(400 \mathrm{MHz}, \mathrm{CDCl}_{3}\right)$

${ }^{13} \mathrm{C}$ NMR $\left(100 \mathrm{MHz}, \mathrm{CDCl}_{3}\right)$

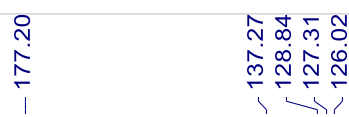

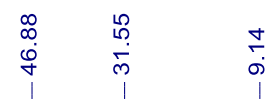

csk-3-23-3.11.fid

C13CPD CDCI3 \{D:INMR400IDNL0604\} nmr-new 22

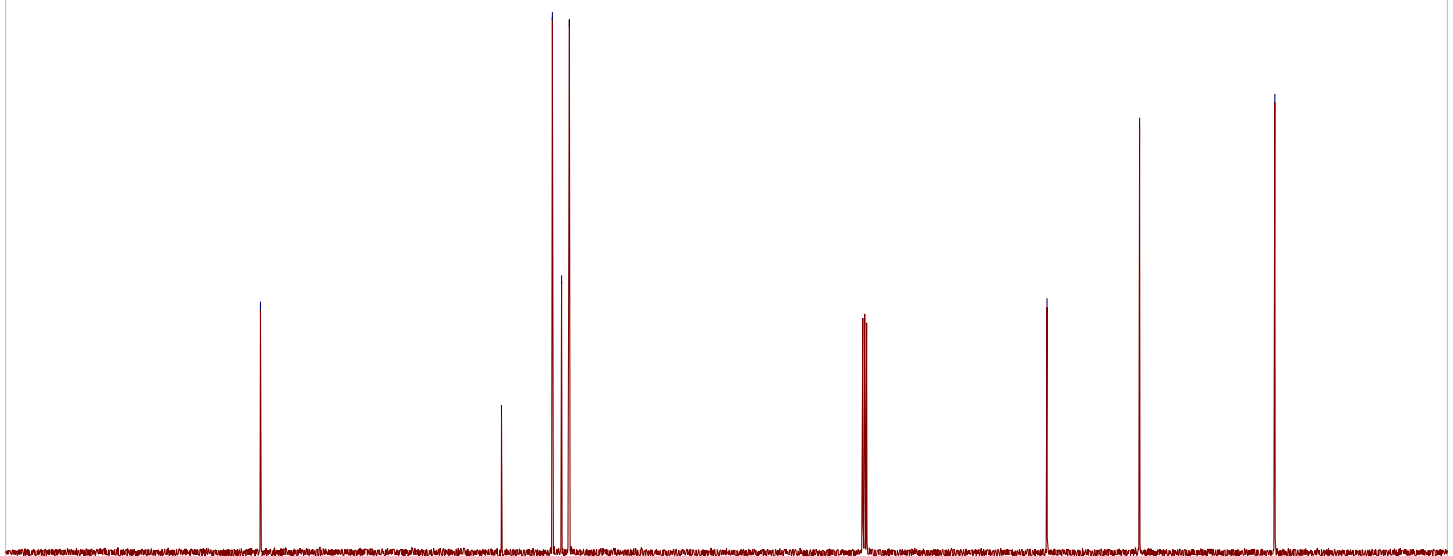

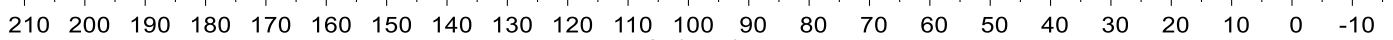
f1 (ppm) 


\begin{tabular}{|c|c|c|}
\hline ల్mల్ & 只 & 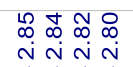 \\
\hline
\end{tabular}

csk-3-23-5.10.fid

PROTON CDCI3 \{D:INMR400IDNL0604\} nmr-new 27
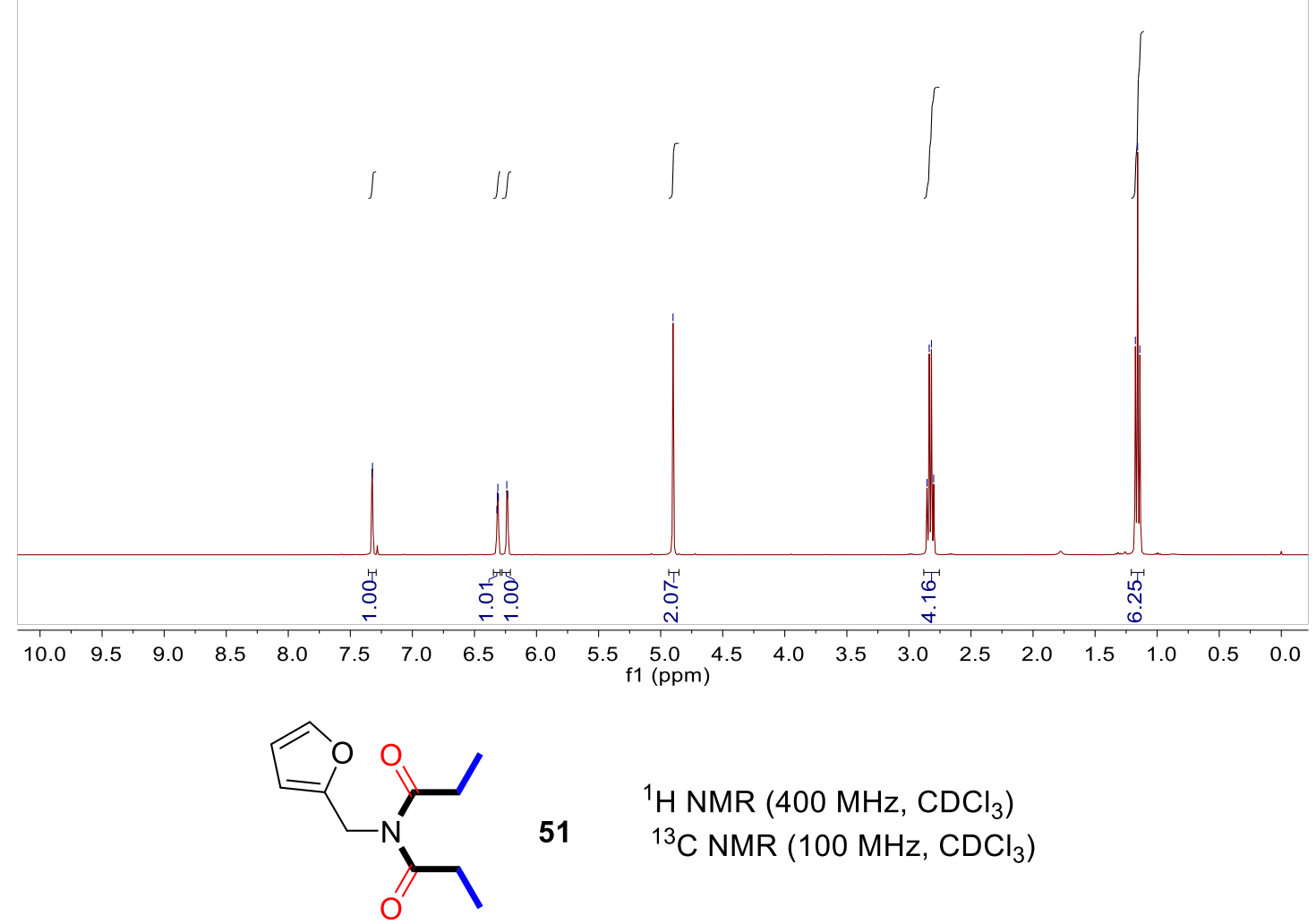

${ }^{1} \mathrm{H}$ NMR $\left(400 \mathrm{MHz}, \mathrm{CDCl}_{3}\right)$

$51 \quad{ }^{13} \mathrm{C} \mathrm{NMR}\left(100 \mathrm{MHz}, \mathrm{CDCl}_{3}\right)$
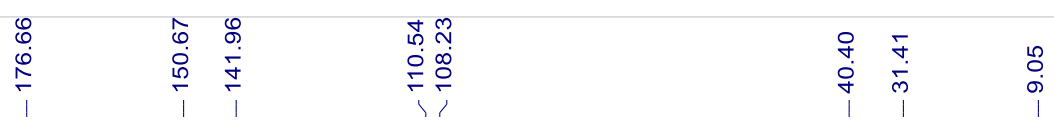

csk-3-23-5.11.fid

C13CPD CDCI3 \{D:INMR400IDNL0604\} nmr-new 27

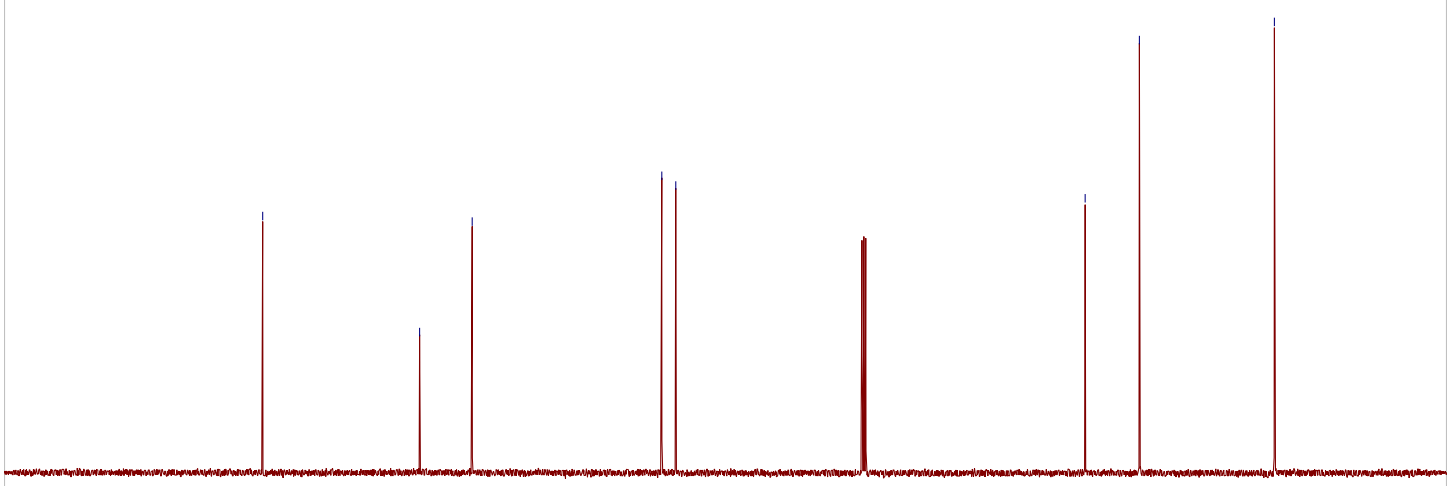

$\begin{array}{lllllllllllllllllllllll}210 & 200 & 190 & 180 & 170 & 160 & 150 & 140 & 130 & 120 & 110 & 100 & 90 & 80 & 70 & 60 & 50 & 40 & 30 & 20 & 10 & 0 & -10\end{array}$ f1 (ppm) 
csk-3-23-8.10.fid

PROTON CDCI3 \{D:INMR400Idnl0604\} nmr-new 58

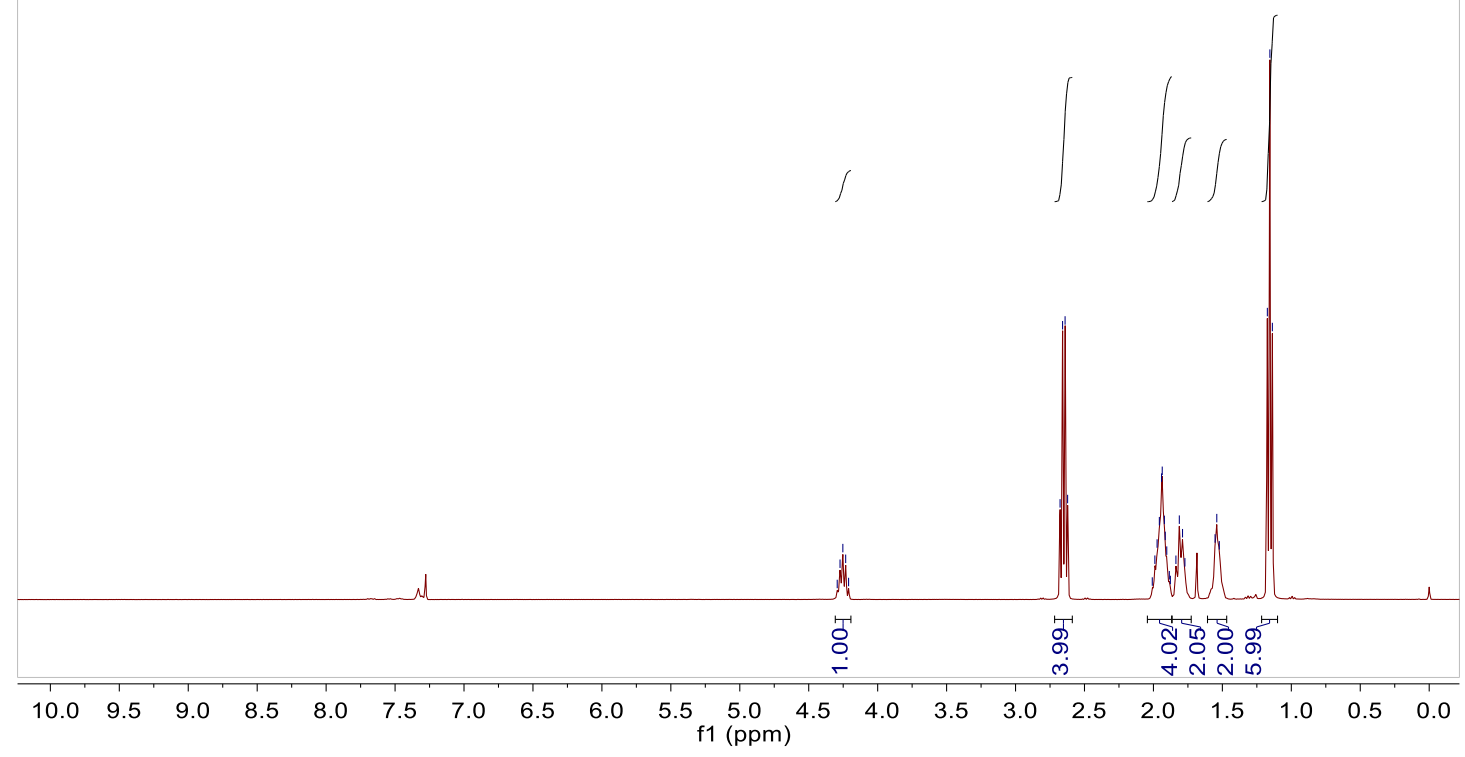<smiles>CCC(=O)N(C(=O)CC)C1CCCC1</smiles>

${ }^{13} \mathrm{C} \mathrm{NMR}\left(100 \mathrm{MHz}, \mathrm{CDCl}_{3}\right)$

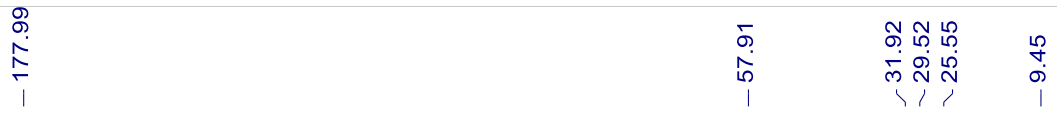

csk-3-23-8.11.fid

C13CPD CDCI3 \{D:INMR400ldn10604\} nmr-new 58

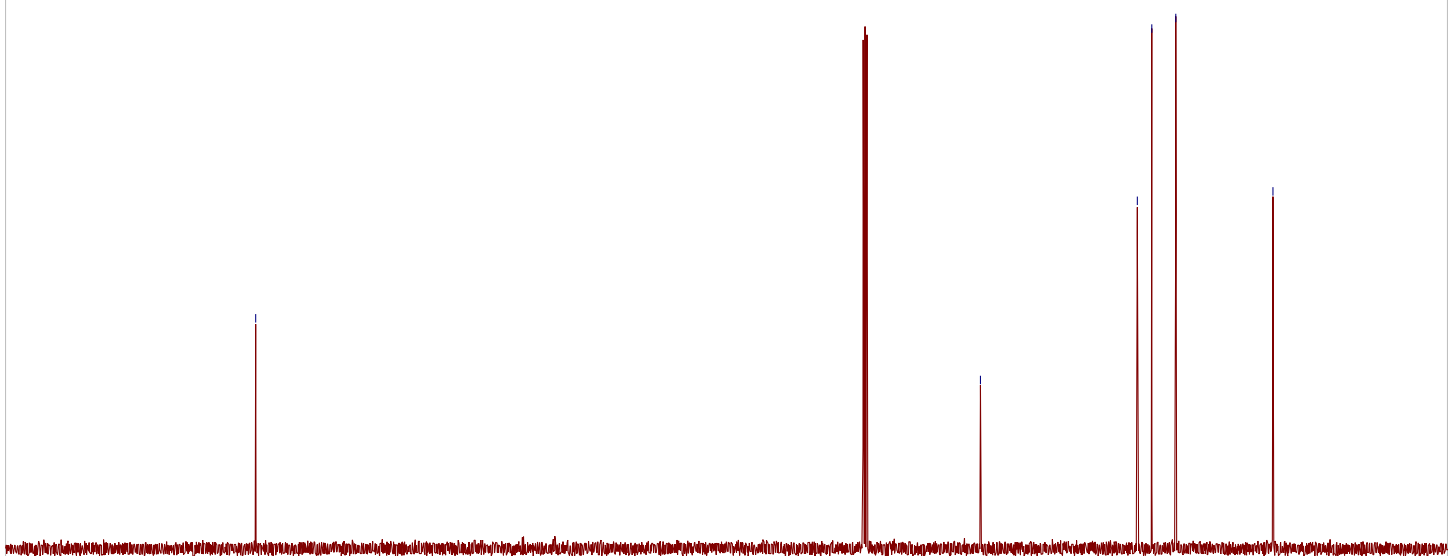

$\begin{array}{lllllllllllllllllllllll}210 & 200 & 190 & 180 & 170 & 160 & 150 & 140 & 130 & 120 & 110 & 100 & 90 & 80 & 70 & 60 & 50 & 40 & 30 & 20 & 10 & 0 & -10\end{array}$ 
csk-3-23-13.10.fid

PROTON CDCI3 \{D:INMR400IDNL0604\} nmr-new 50
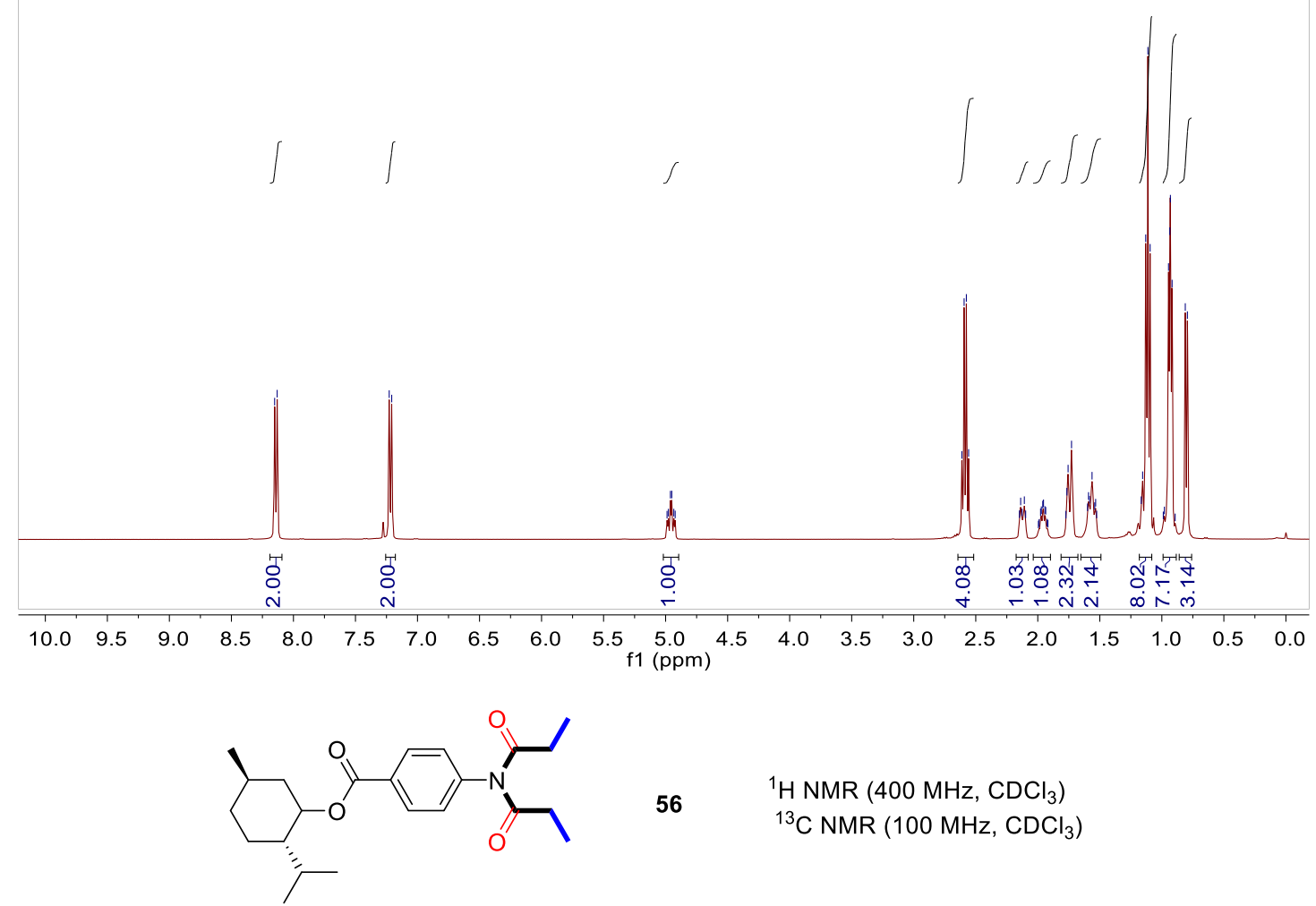

$56 \quad{ }^{1} \mathrm{H} \mathrm{NMR}\left(400 \mathrm{MHz}, \mathrm{CDCl}_{3}\right)$

${ }^{13} \mathrm{C} \mathrm{NMR}\left(100 \mathrm{MHz}, \mathrm{CDCl}_{3}\right)$

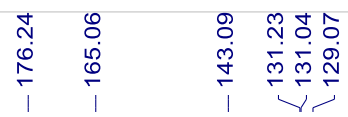

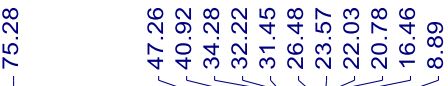

csk-3-23-13.11.fid

C13CPD CDCI3 \{D:INMR400IDNL0604\} nmr-new 50

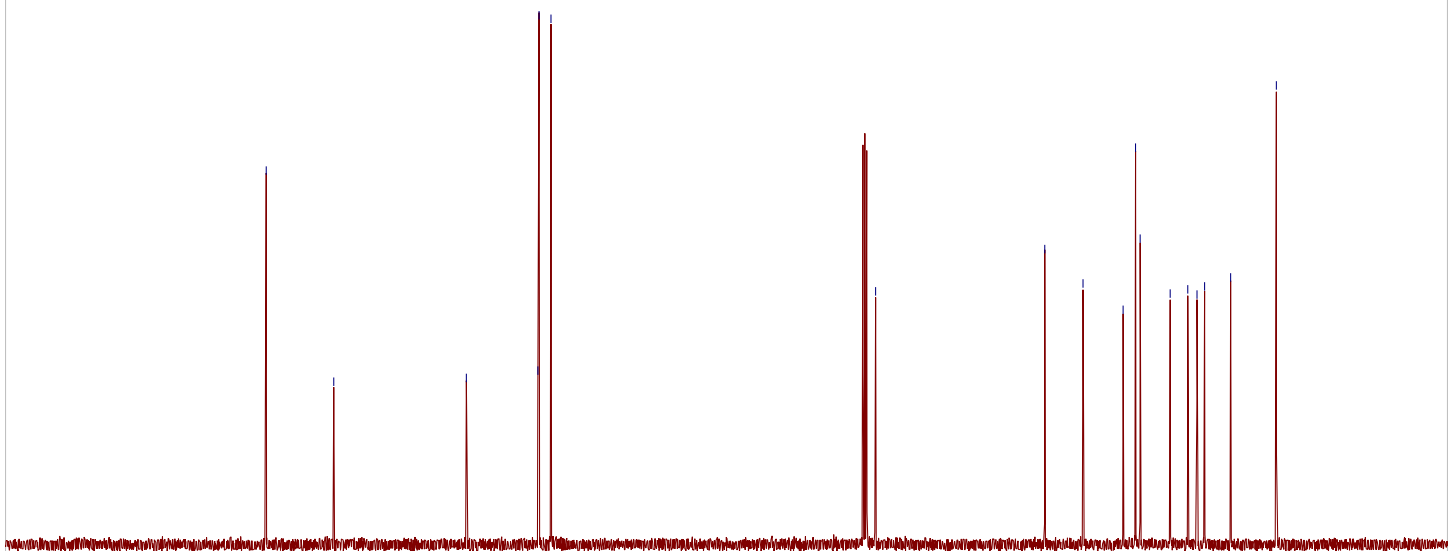

$\begin{array}{lllllllllllllllllllllll}210 & 200 & 190 & 180 & 170 & 160 & 150 & 140 & 130 & 120 & \begin{array}{c}110 \\ \mathrm{f} 1\end{array}\left(\begin{array}{ll}100 \\ (\mathrm{ppm})\end{array}\right. & 90 & 80 & 70 & 60 & 50 & 40 & 30 & 20 & 10 & 0 & -10\end{array}$ 
csk-3-23-14.14.fid

PROTON CDCI3 \{D:INMR400ldnl0604\} nmr-new 48
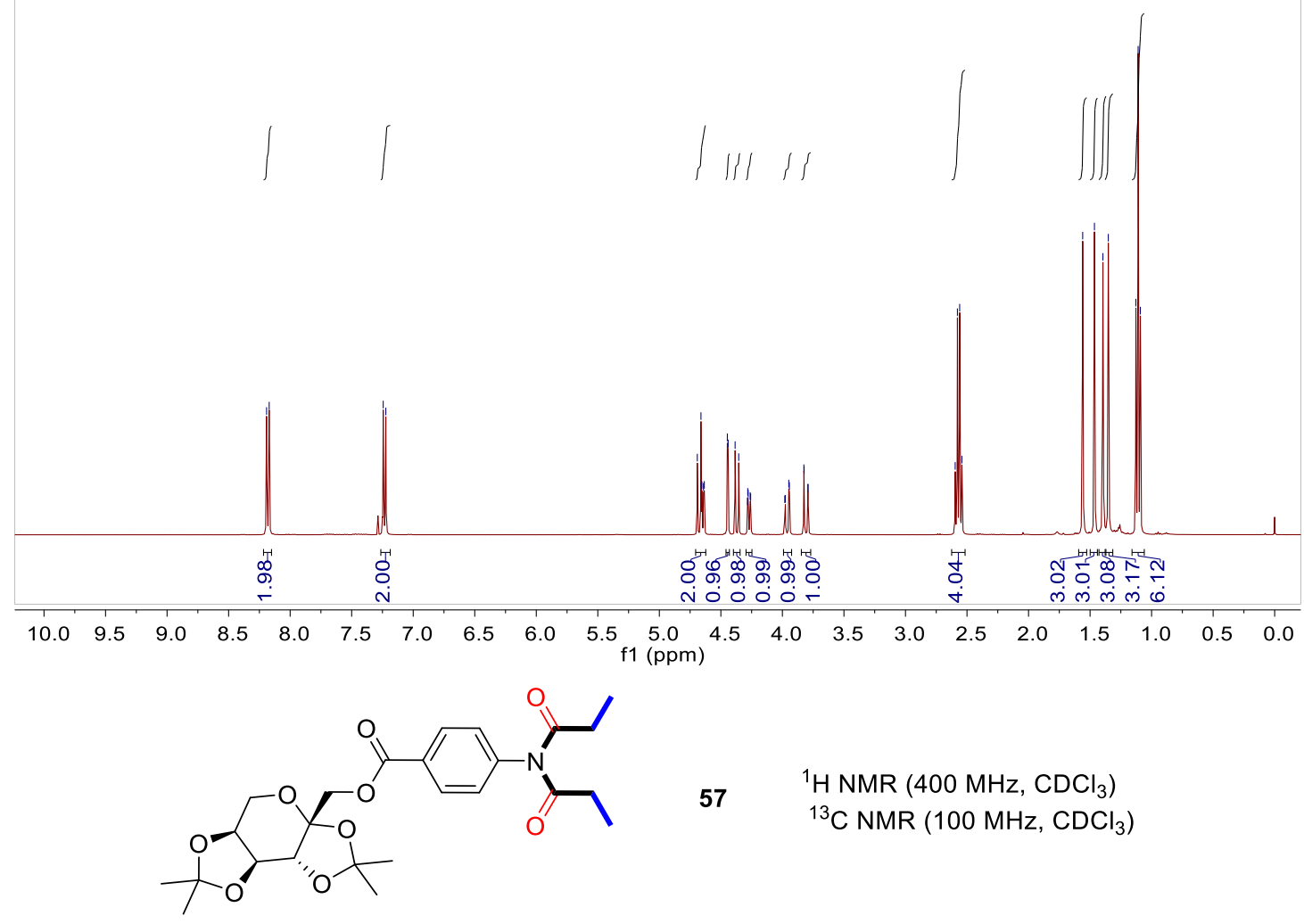

$57 \quad{ }^{1} \mathrm{H}$ NMR $\left(400 \mathrm{MHz}, \mathrm{CDCl}_{3}\right)$

\begin{tabular}{|c|c|c|c|c|}
\hline $\begin{array}{l}N \\
0 \\
0 \\
0 \\
\end{array}$ & 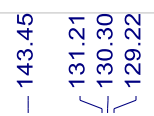 & 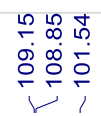 & 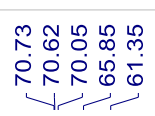 & 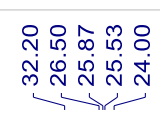 \\
\hline
\end{tabular}

csk-3-23-14.15.fid

C13CPD CDCI3 \{D:INMR400ldnl0604\} nmr-new 48

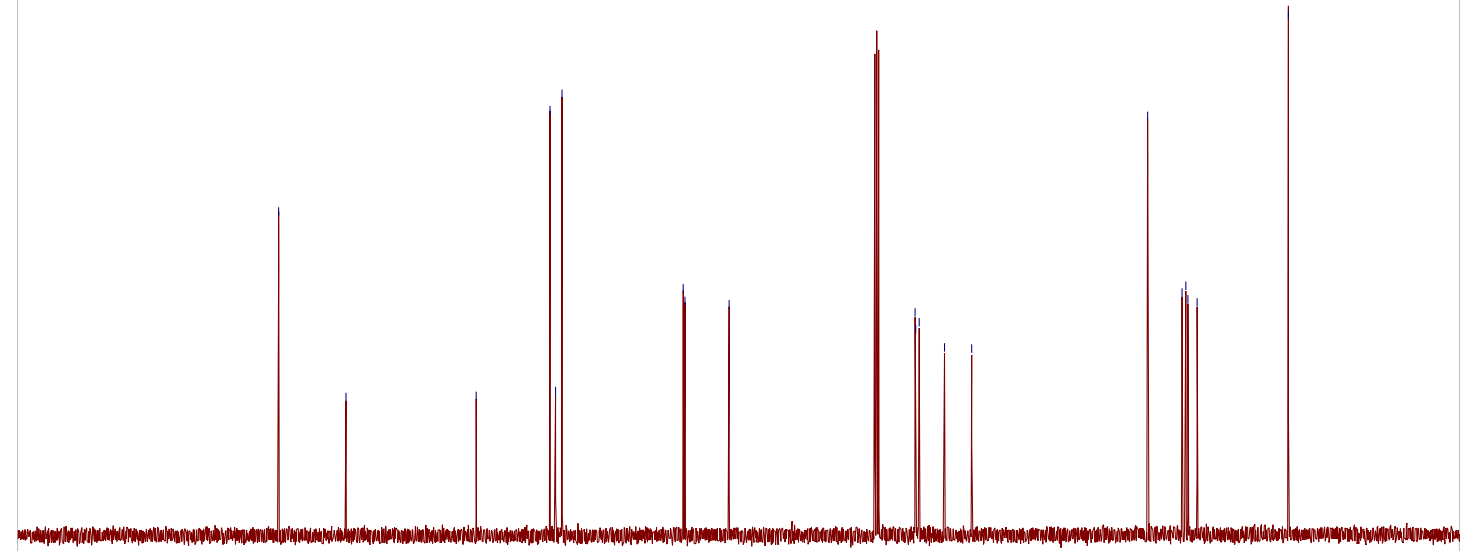

$\begin{array}{lllllllllllllllllllllll}210 & 200 & 190 & 180 & 170 & 160 & 150 & 140 & 130 & 120 & 110 & 100 & 90 & 80 & 70 & 60 & 50 & 40 & 30 & 20 & 10 & 0 & -10\end{array}$ 


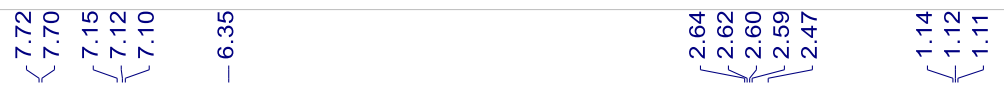

csk-3-12-5.10.fid

PROTON CDCI3 \{D:INMR400IDNL0604\} nmr-new 32
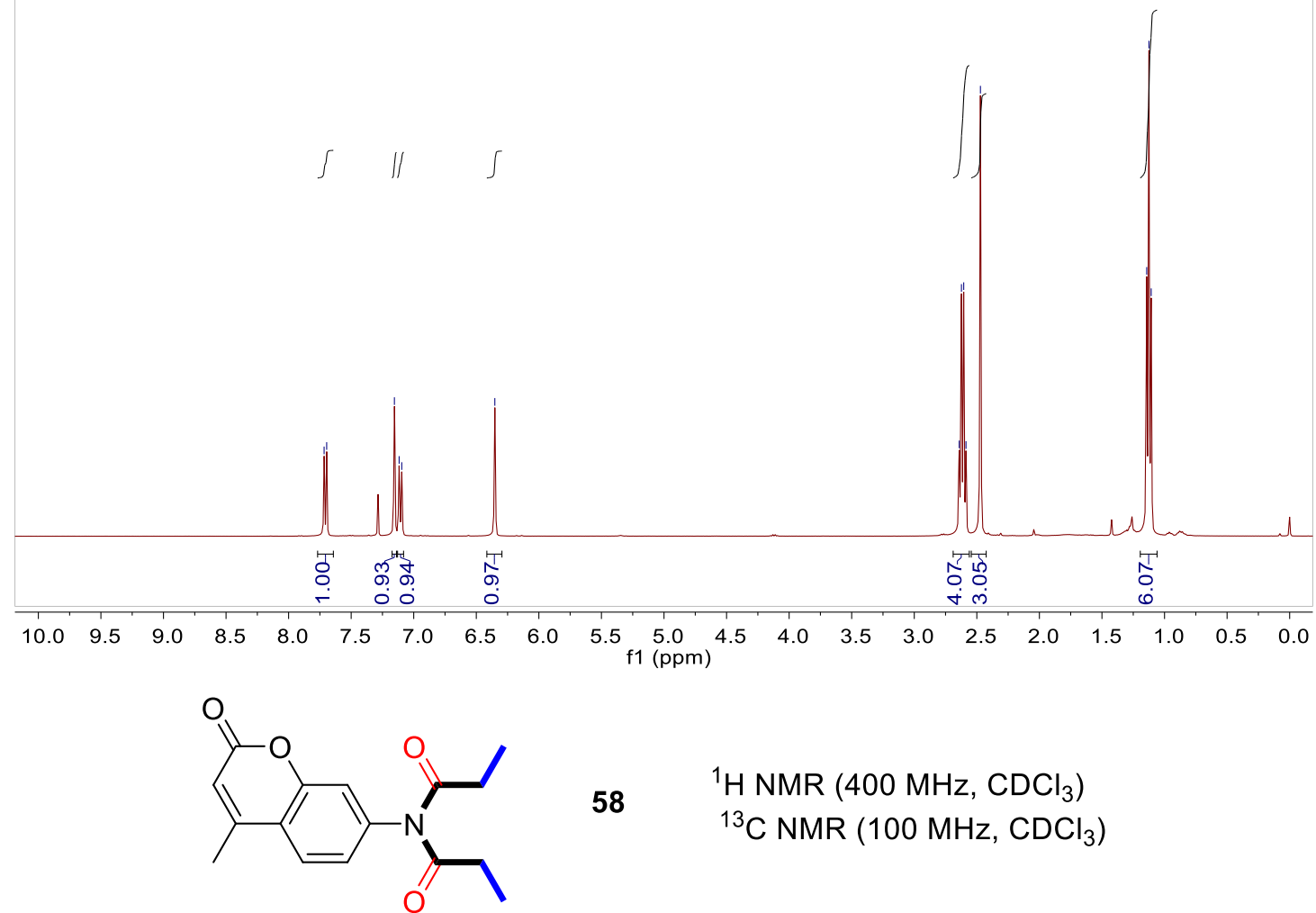

$58 \quad{ }^{1} \mathrm{H}$ NMR $\left(400 \mathrm{MHz}, \mathrm{CDCl}_{3}\right)$ ${ }^{13} \mathrm{C}$ NMR $\left(100 \mathrm{MHz}, \mathrm{CDCl}_{3}\right)$

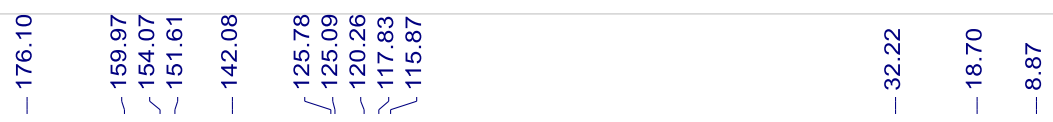

csk-3-12-5.11.fid

C13CPD CDCI3 \{D:INMR400IDNL0604\} nmr-new 32

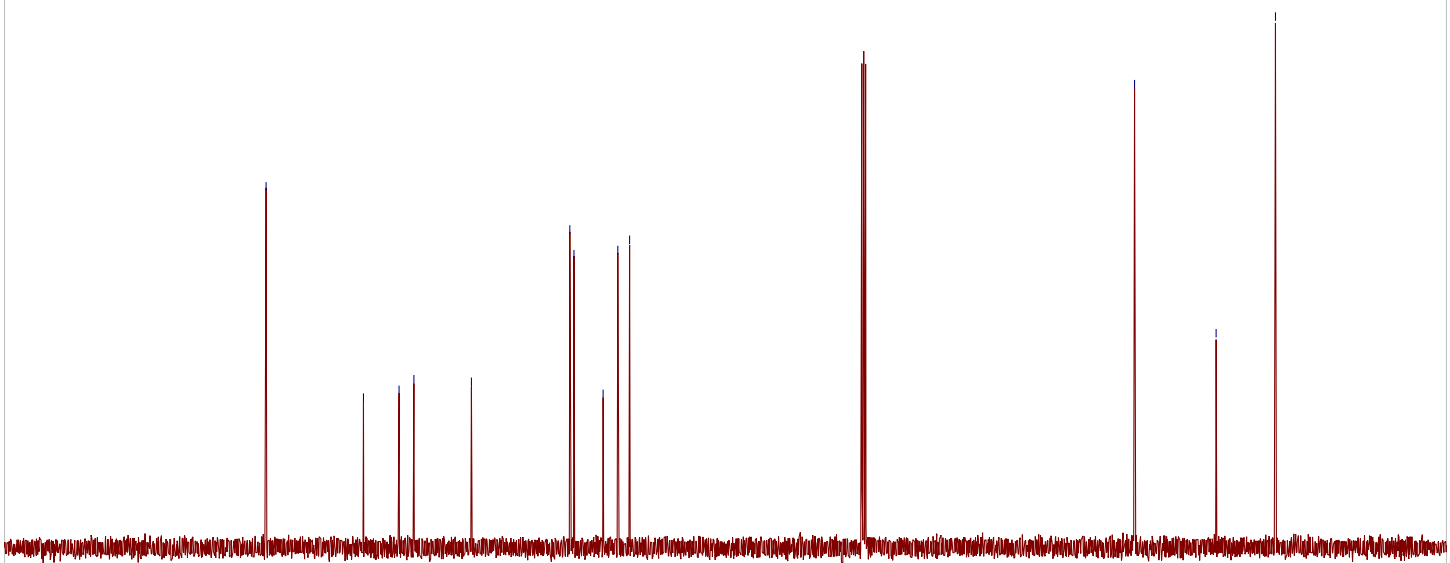

$\begin{array}{lllllllllllll}210 & 200 & 190 & 180 & 170 & 160 & 150 & 140 & 130 & 120 & 110 & 100 & 9\end{array}$ f1 (ppm) 
csk-3-23-15.10.fid

PROTON CDCI3 \{D:INMR400IDNL0604\} nmr-new 52
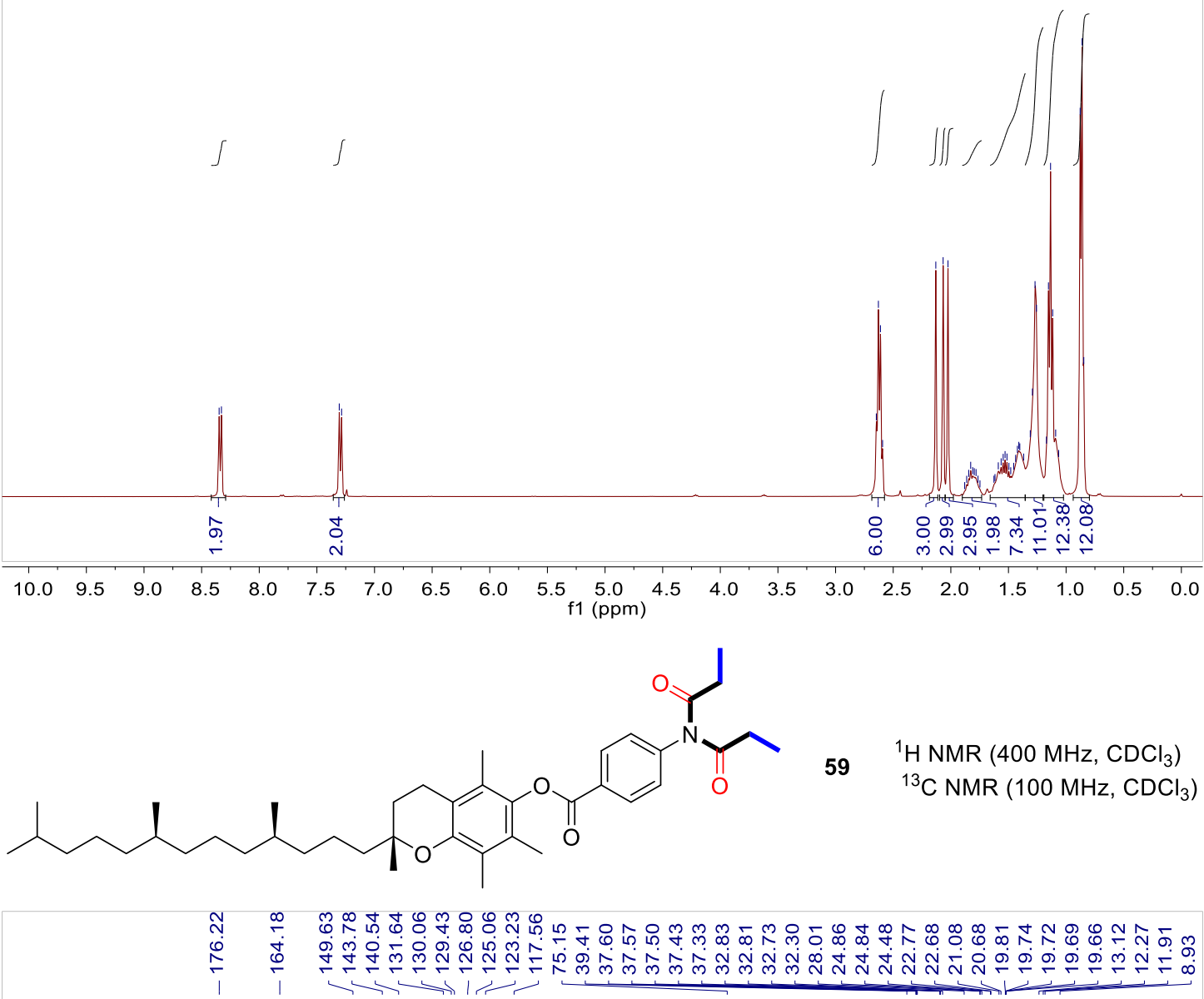

csk-3-23-15.11.fid

C13CPD CDCI3 \{D:INMR400|DNL0604\} nmr-new 52

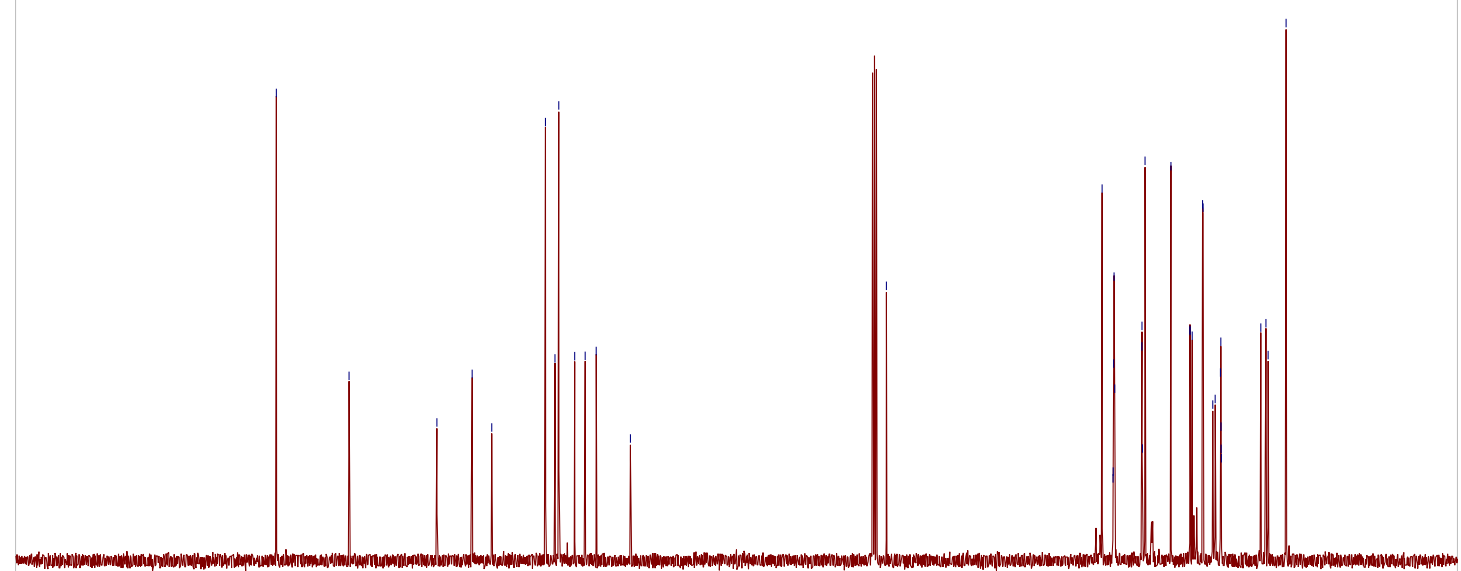

$\begin{array}{lllllllllllllllllllllll}210 & 200 & 190 & 180 & 170 & 160 & 150 & 140 & 130 & 120 & 110 & 100 & 90 & 80 & 70 & 60 & 50 & 40 & 30 & 20 & 10 & 0 & -10\end{array}$ 
csk-3-12-4.20.fid

PROTON CDCI3 \{D:INMR400ldnl0604\} nmr-new 46
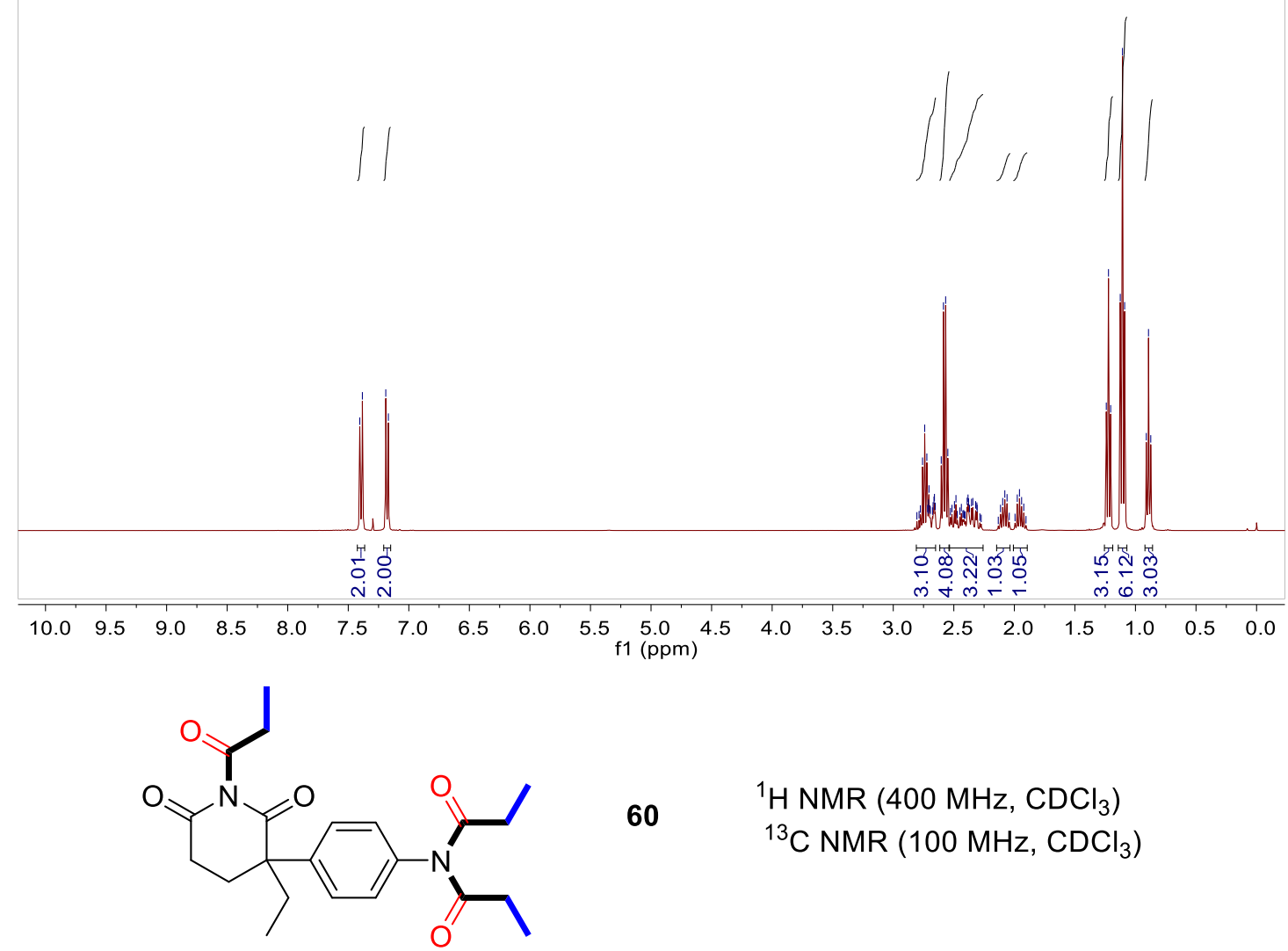

60

${ }^{1} \mathrm{H}$ NMR $\left(400 \mathrm{MHz}, \mathrm{CDCl}_{3}\right)$

${ }^{13} \mathrm{C}$ NMR $\left(100 \mathrm{MHz}, \mathrm{CDCl}_{3}\right)$

\section{ब.}

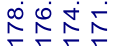

กิช ชิำ

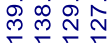

ลุ

ঢ.

厉

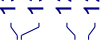

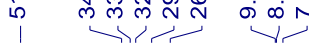

csk-3-12-4.21.fid

C13CPD CDCI3 \{D:INMR400ldnl0604\} nmr-new 46

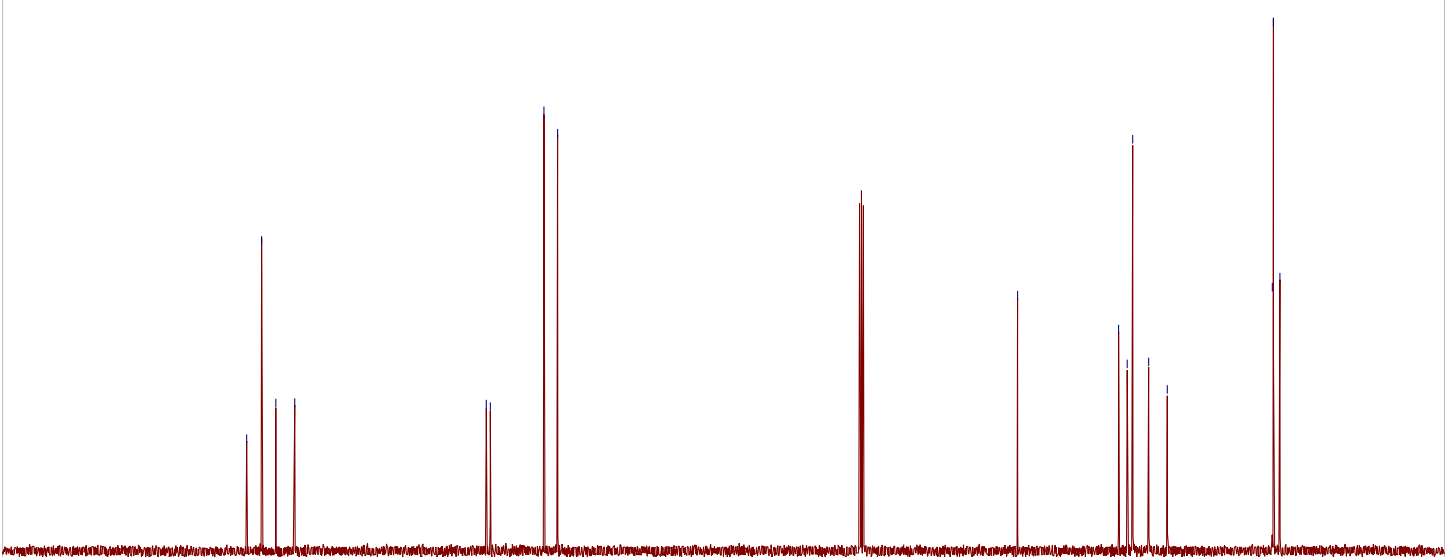

$\begin{array}{lllllllllllllllllllllll}210 & 200 & 190 & 180 & 170 & 160 & 150 & 140 & 130 & 120 & 110 & 100 & 90 & 80 & 70 & 60 & 50 & 40 & 30 & 20 & 10 & 0 & -10\end{array}$ 
csk-3-23-6.10.fid

PROTON CDCI3 \{D:INMR400IDNL0604\} nmr-new 28
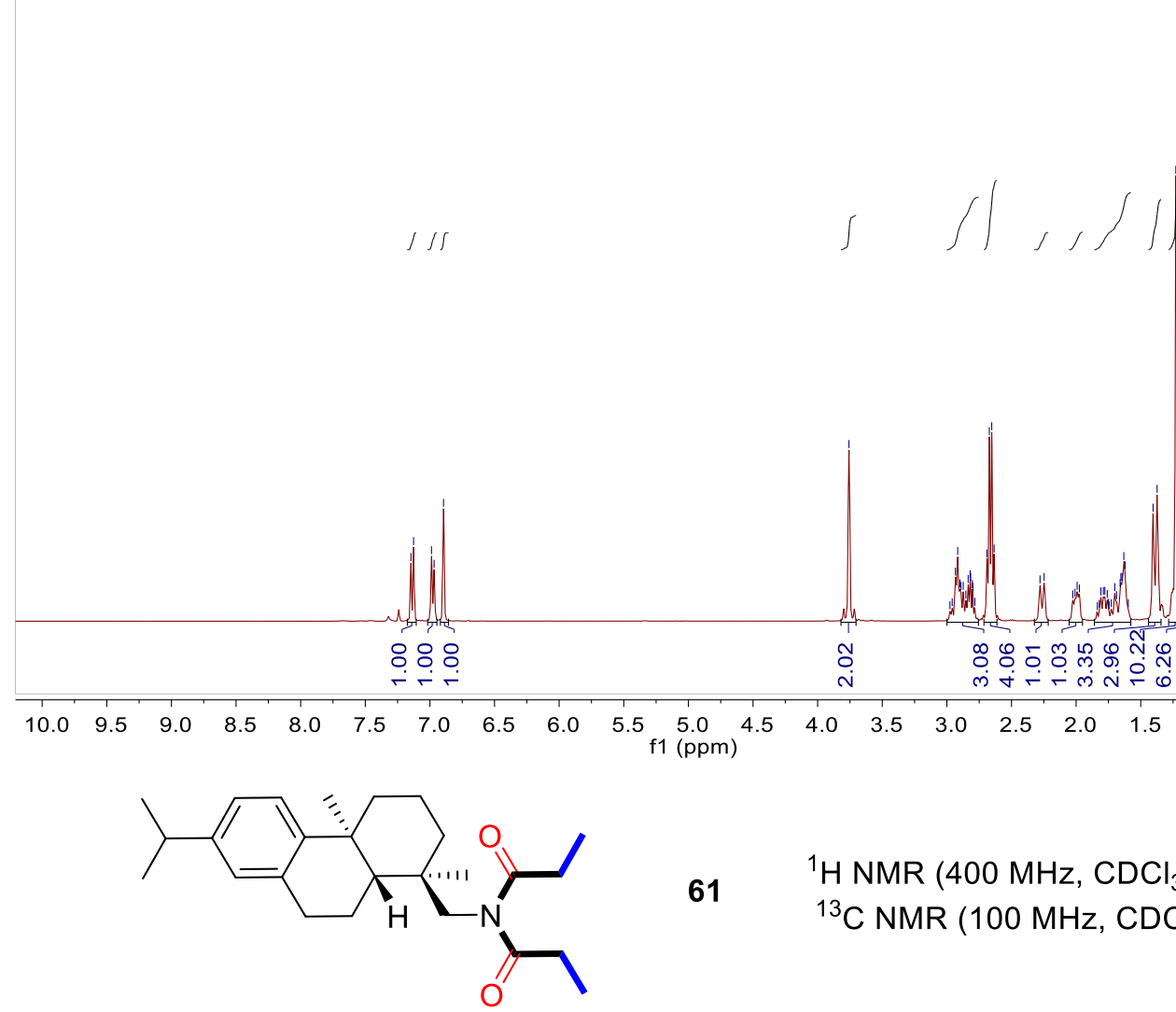

61

${ }^{1} \mathrm{H}$ NMR $\left(400 \mathrm{MHz}, \mathrm{CDCl}_{3}\right)$

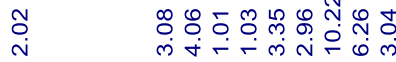

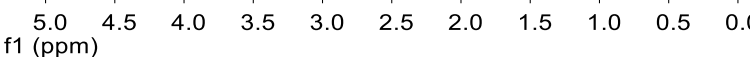

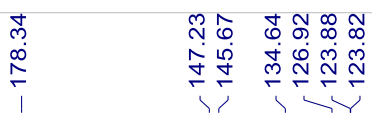

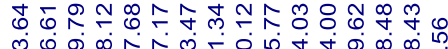
نூ

csk-3-23-6.11.fid

C13CPD CDCI3 \{D:INMR400IDNL0604\} nmr-new 28

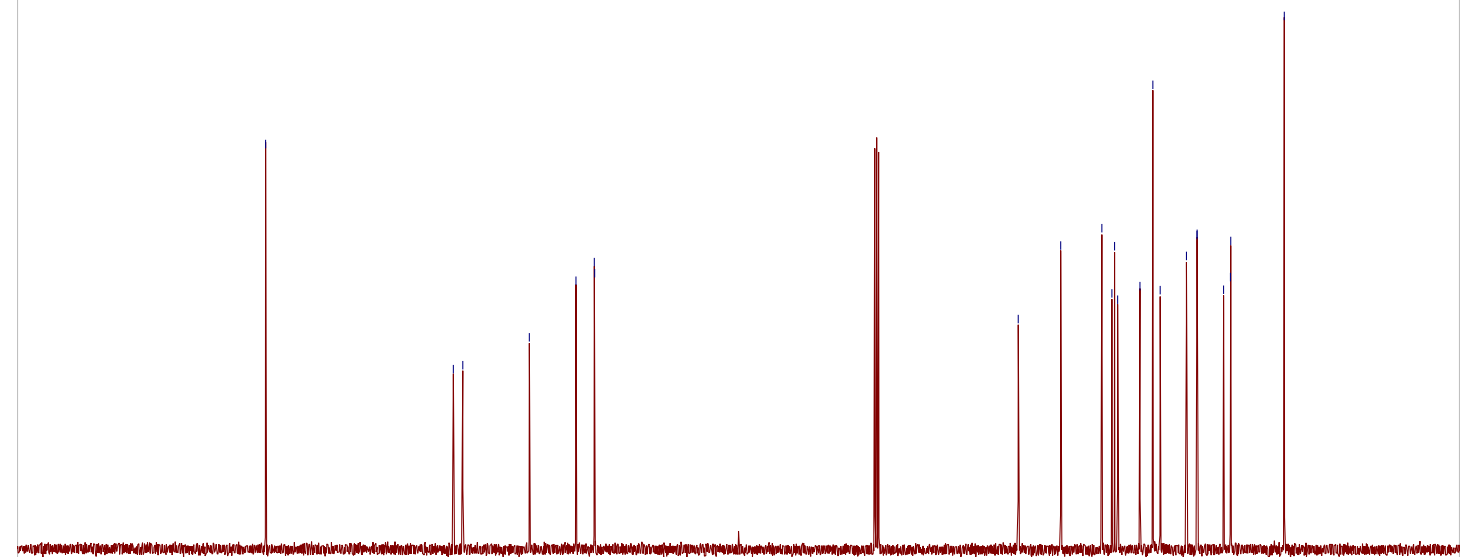

$\begin{array}{llllllllllllllllllllll}210 & 200 & 190 & 180 & 170 & 160 & 150 & 140 & 130 & 120 & \begin{array}{c}110 \\ \mathrm{f} 1\end{array}(\mathrm{ppm}) \\ (\mathrm{ppo}) & 90 & 80 & 70 & 60 & 50 & 40 & 30 & 20 & 10 & 0 & -10\end{array}$ 


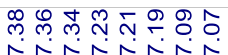 \\ rivinision}

울 우ำ

4niv

N

CSK-2-23-11.10.fid

PROTON CDCI3 \{D:INMR400IDNL0604\} nmr-new 20
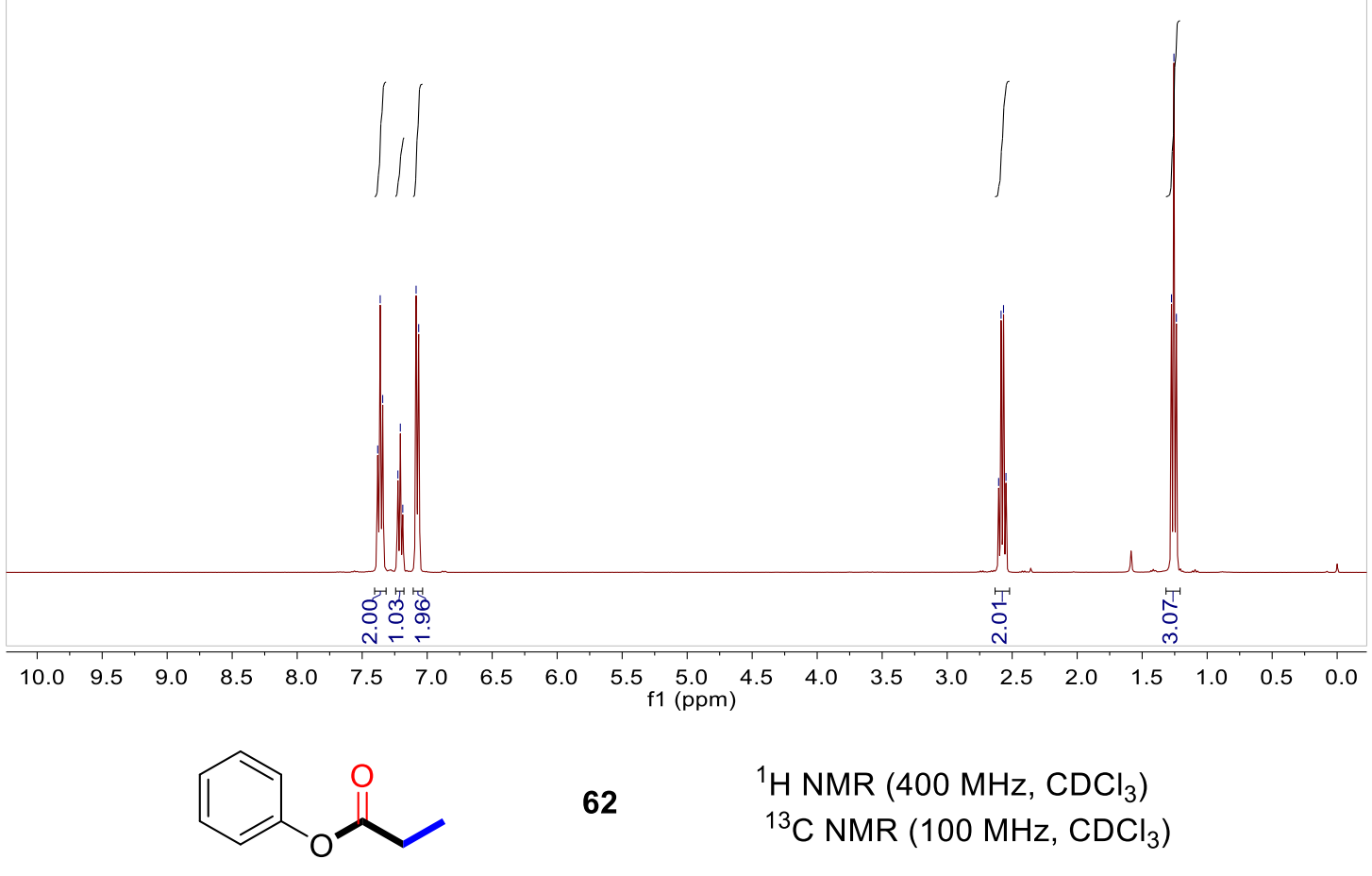

62

${ }^{1} \mathrm{H}$ NMR $\left(400 \mathrm{MHz}, \mathrm{CDCl}_{3}\right)$

${ }^{13} \mathrm{C}$ NMR $\left(100 \mathrm{MHz}, \mathrm{CDCl}_{3}\right)$

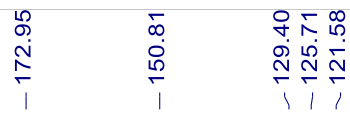

CSK-2-23-11.11.fid

C13CPD CDCI3 \{D:INMR400IDNL0604\} nmr-new 20

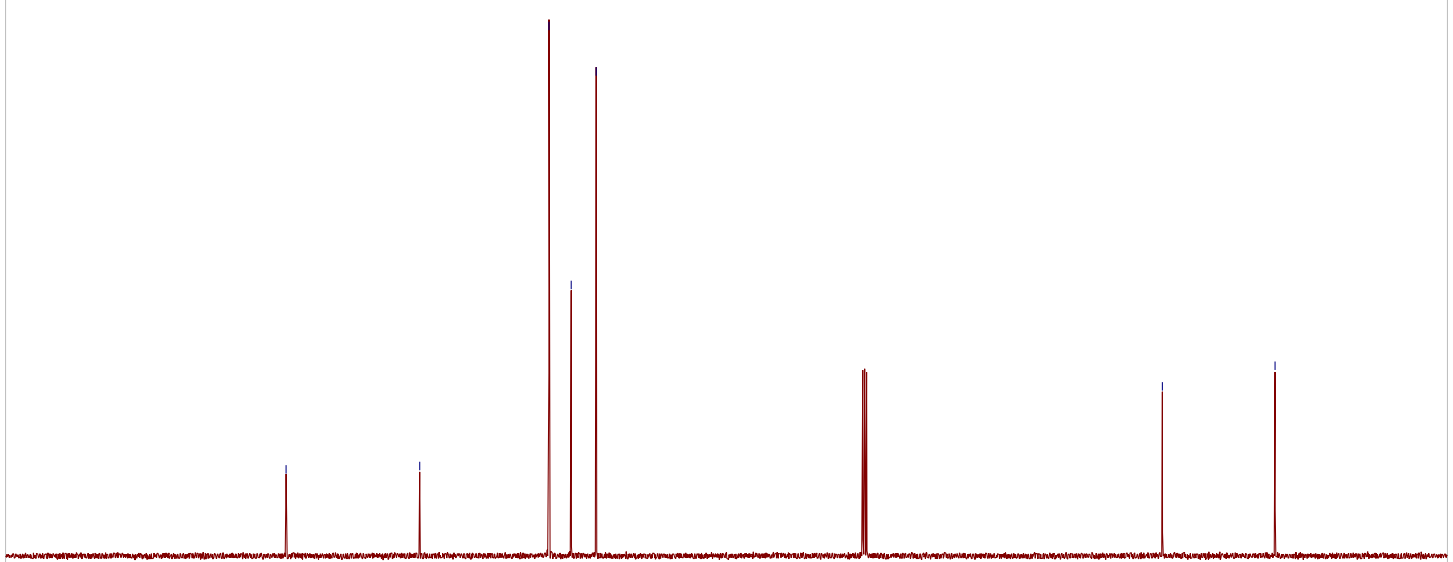

$\begin{array}{lllllllllllllllllllllll}210 & 200 & 190 & 180 & 170 & 160 & 150 & 140 & 130 & 120 & 110 & 100 & 90 & 80 & 70 & 60 & 50 & 40 & 30 & 20 & 10 & 0 & -10\end{array}$ 


\begin{tabular}{|c|c|c|}
\hline 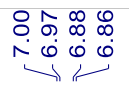 & $\begin{array}{l}\stackrel{0}{0} \\
\stackrel{m}{1} \\
1\end{array}$ & 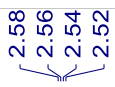 \\
\hline
\end{tabular}

csk-3-33-3.10.fid

PROTON CDCI3 \{D:INMR400IDNL0604\} nmr-new 16
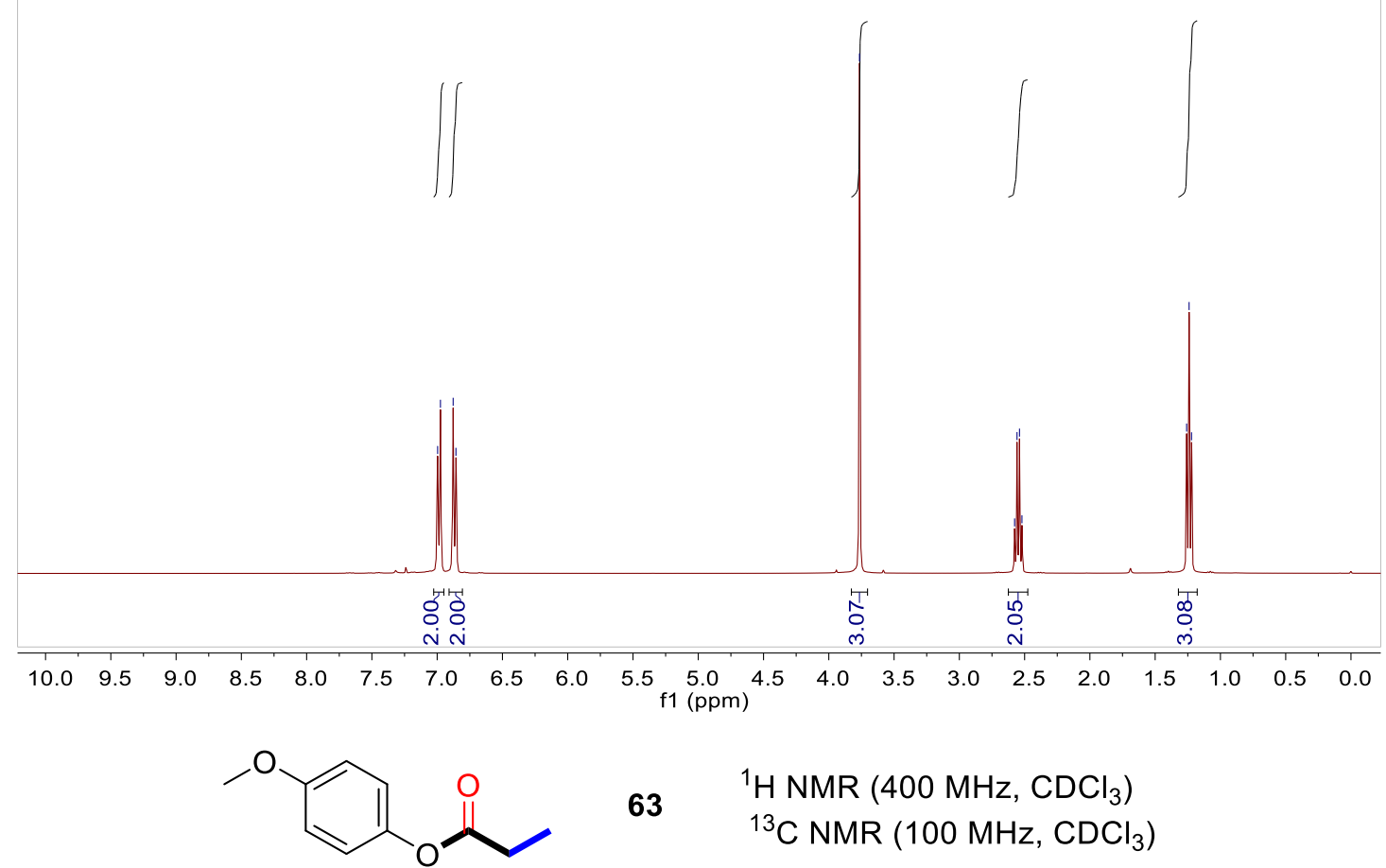

63

${ }^{1} \mathrm{H}$ NMR $\left(400 \mathrm{MHz}, \mathrm{CDCl}_{3}\right)$

${ }^{13} \mathrm{C}$ NMR $\left(100 \mathrm{MHz}, \mathrm{CDCl}_{3}\right)$

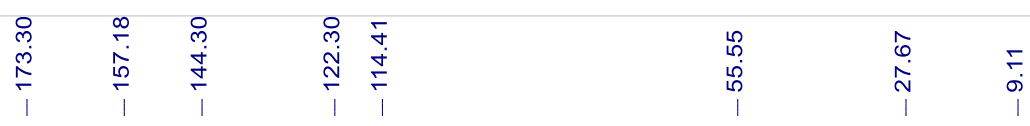

sk-3-33-3.11.fid

C13CPD CDCI3 \{D:INMR400IDNL0604\} nmr-new 16

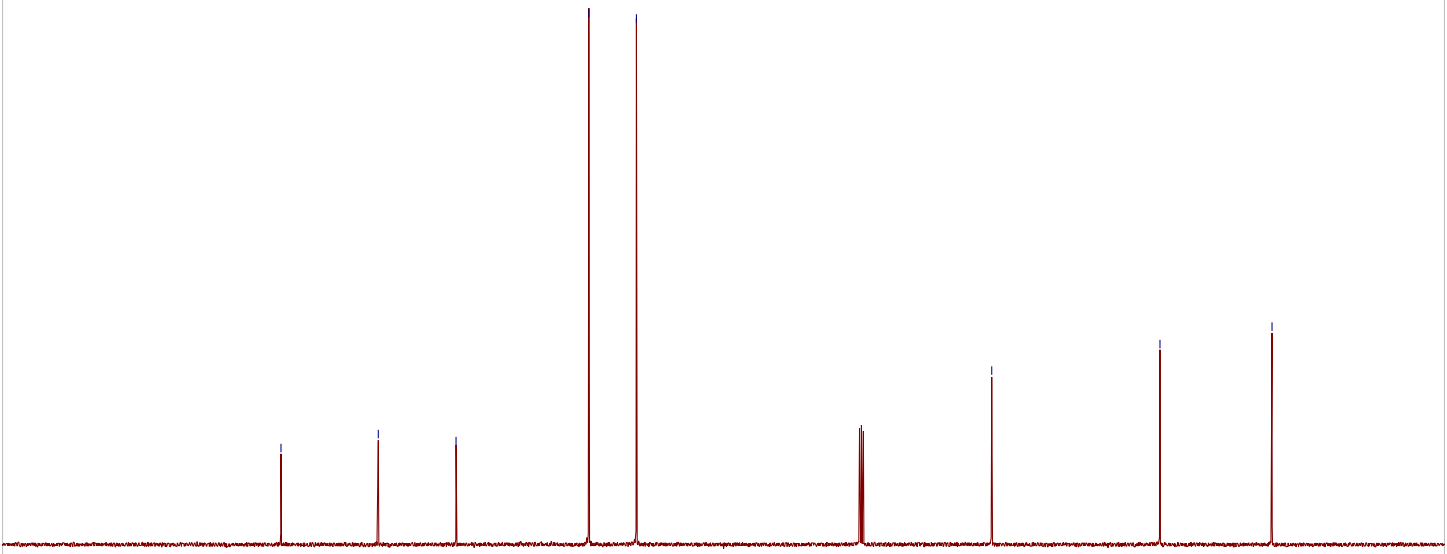

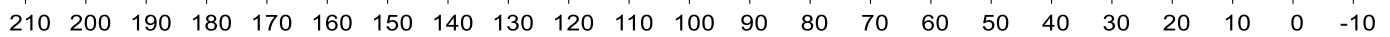
f1 (ppm) 

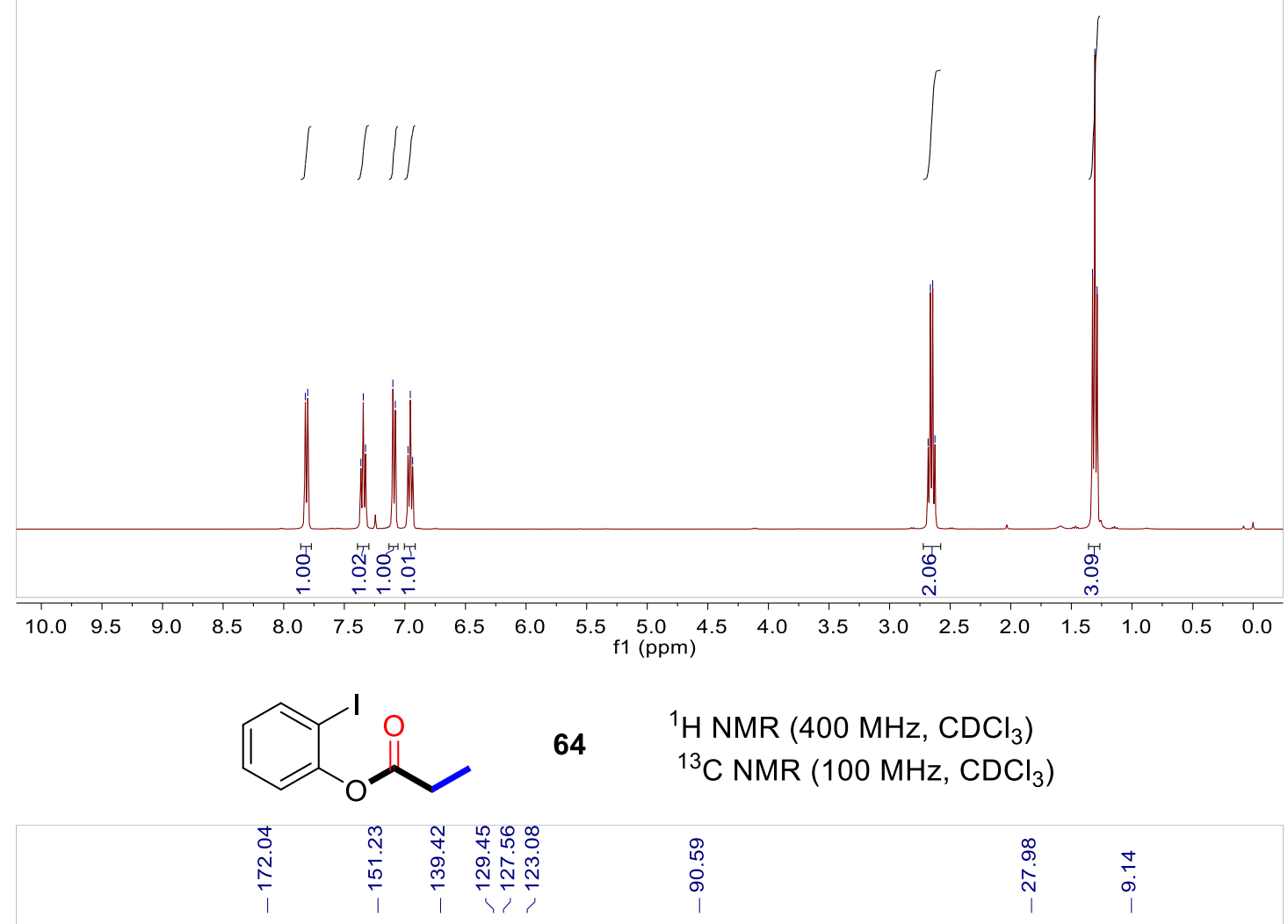

csk-3-33-5.11.fid

C13CPD CDCI3 \{D:INMR400IDNL0604\} nmr-new 18

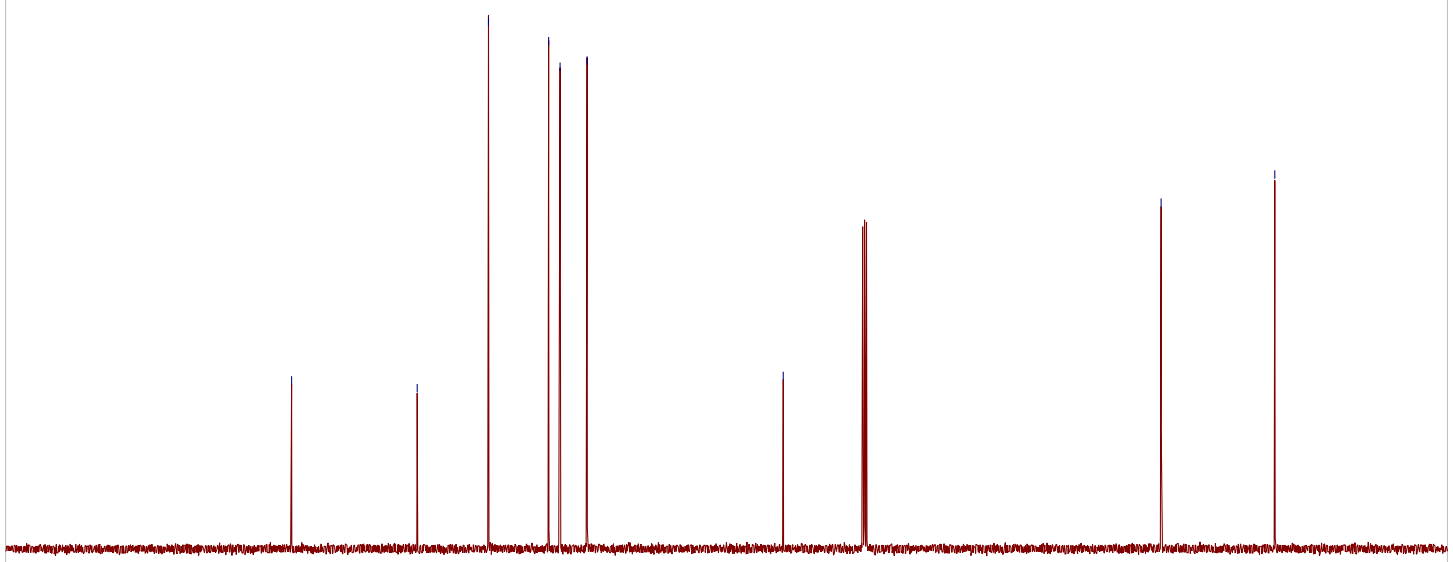

$\begin{array}{lllllllllllllllllllllll}210 & 200 & 190 & 180 & 170 & 160 & 150 & 140 & 130 & 120 & 110 & 100 & 90 & 80 & 70 & 60 & 50 & 40 & 30 & 20 & 10 & 0 & -10\end{array}$ 


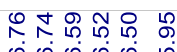 \\ $\dot{0} \dot{0} \dot{0} \dot{0} \dot{0}$ in

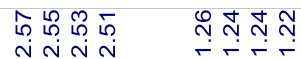 \\ $\sqrt{64 \sin }$}

csk-3-33-4.10.fid

PROTON CDCI3 \{D:INMR400IDNL0604\} nmr-new 17
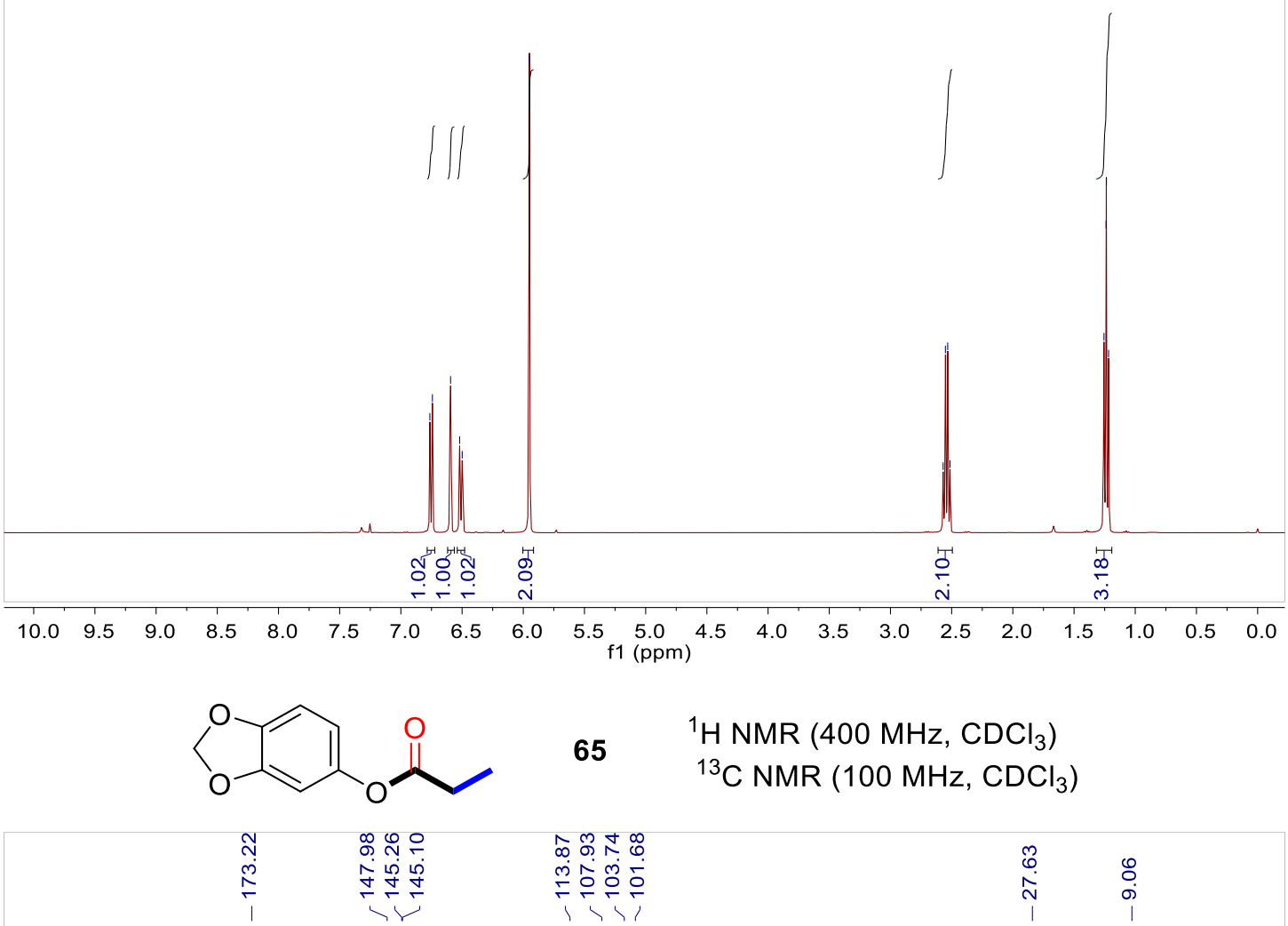

${ }^{1} \mathrm{H}$ NMR $\left(400 \mathrm{MHz}, \mathrm{CDCl}_{3}\right)$

${ }^{13} \mathrm{C} \mathrm{NMR}\left(100 \mathrm{MHz}, \mathrm{CDCl}_{3}\right)$

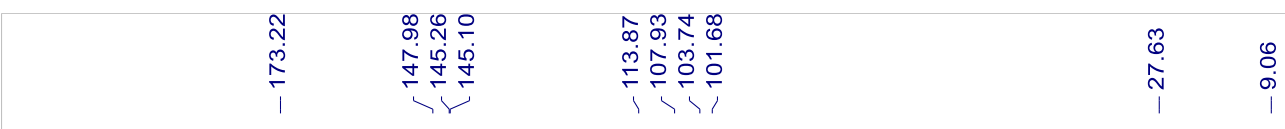

csk-3-33-4.11. fid

C13CPD CDCI3 \{D:INMR400IDNL0604\} nmr-new 17

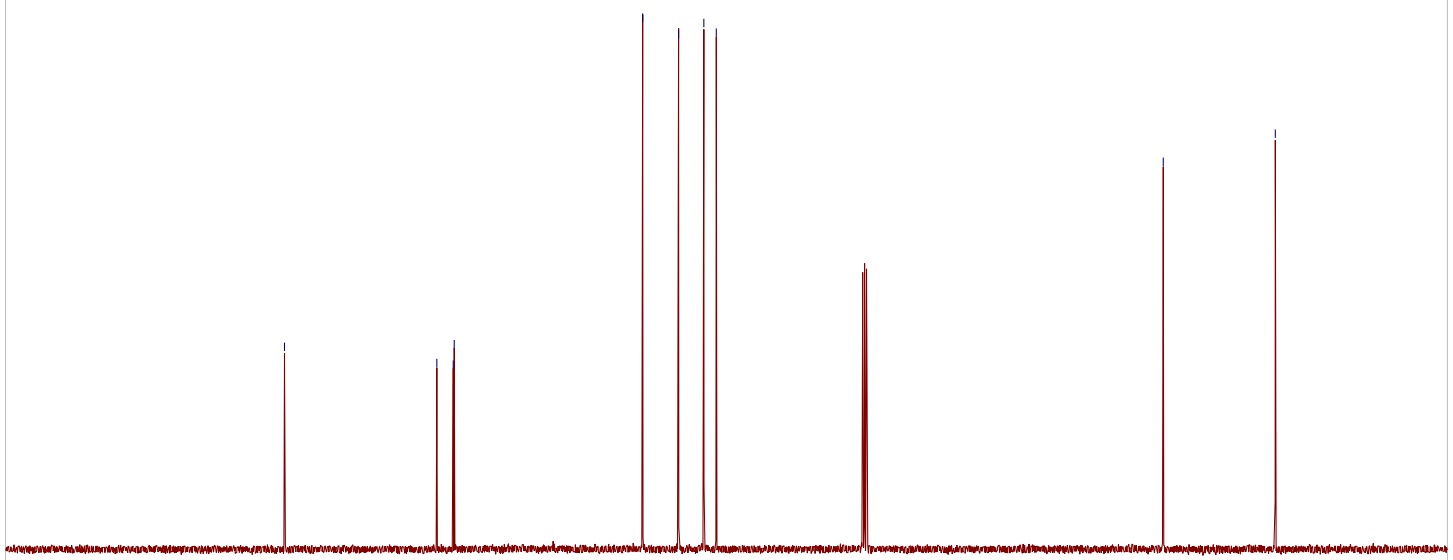

$\begin{array}{llllllllllllllllllllllll}210 & 200 & 190 & 180 & 170 & 160 & 150 & 140 & 130 & 120 & 110 & 100 & 90 & 80 & 70 & 60 & 50 & 40 & 30 & 20 & 10 & 0 & -10\end{array}$ 
CSK-3-33-12.10.fid

PROTON CDCI3 \{D:INMR400IDNL0604\} nmr-new 3
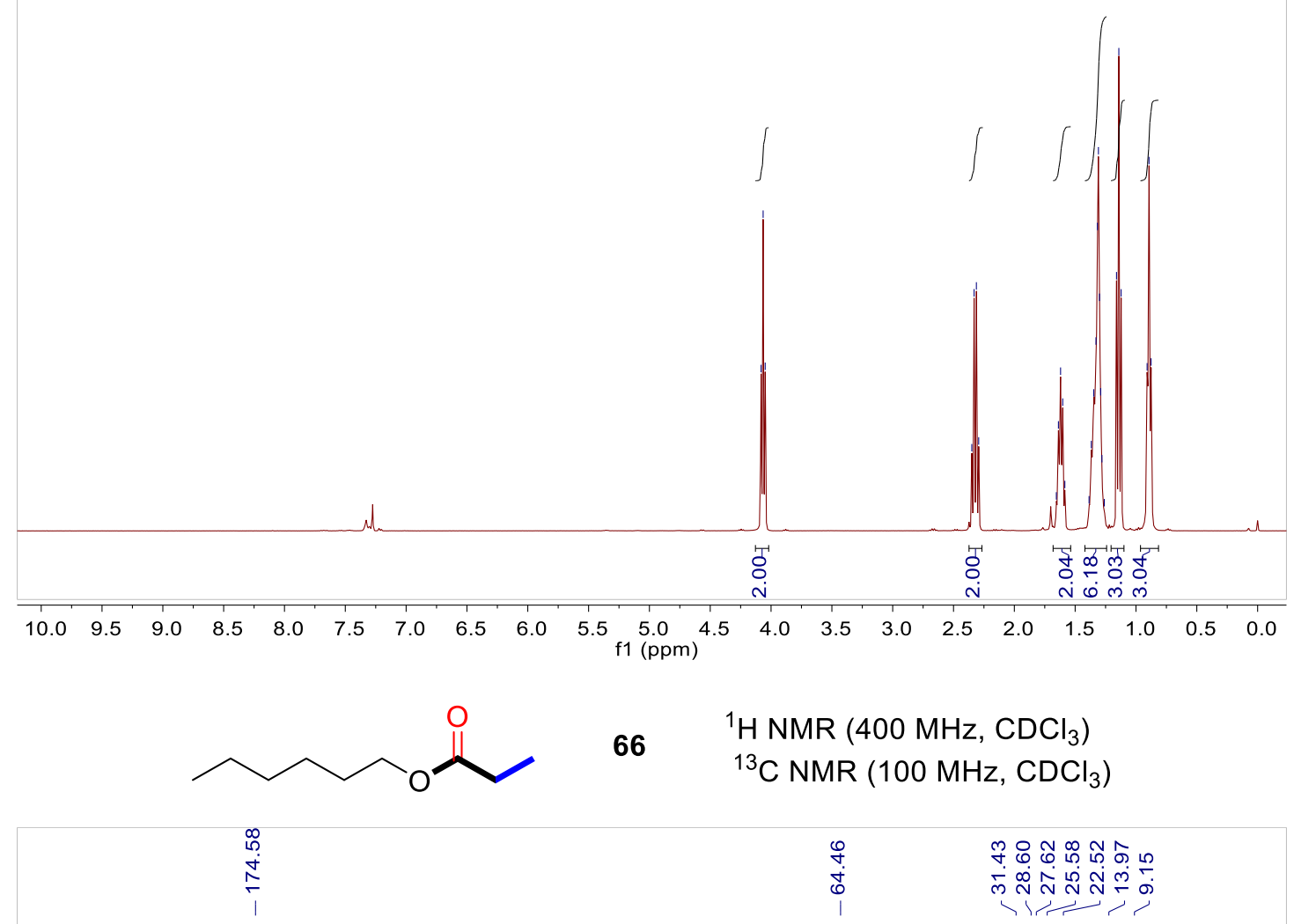

66

${ }^{1} \mathrm{H} \mathrm{NMR}\left(400 \mathrm{MHz}, \mathrm{CDCl}_{3}\right)$
${ }^{13} \mathrm{C} \mathrm{NMR}\left(100 \mathrm{MHz}, \mathrm{CDCl}_{3}\right)$

CSK-3-33-12.11.fid

C13CPD CDCI3 \{D:INMR400IDNL0604\} nmr-new 3

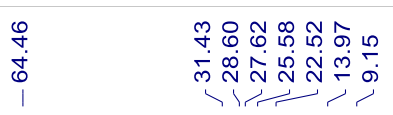

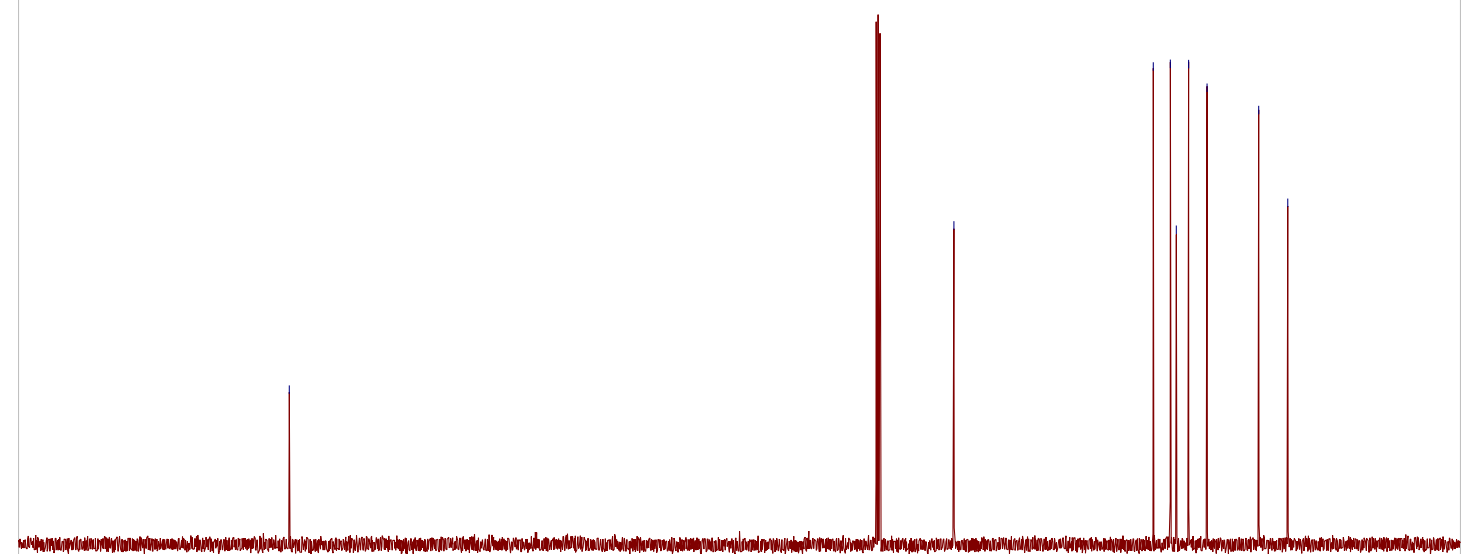

$\begin{array}{lllllllllllllllllllllll}210 & 200 & 190 & 180 & 170 & 160 & 150 & 140 & 130 & 120 & 110 & 100 & 90 & 80 & 70 & 60 & 50 & 40 & 30 & 20 & 10 & 0 & -10\end{array}$ 


\begin{tabular}{|c|c|c|}
\hline 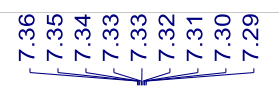 & $\underset{i}{\stackrel{5}{i}}$ & 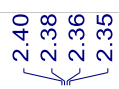 \\
\hline
\end{tabular}

CSK-2-23-12.10.fid

PROTON CDCI3 \{D:INMR400IDNL0604\} nmr-new 21
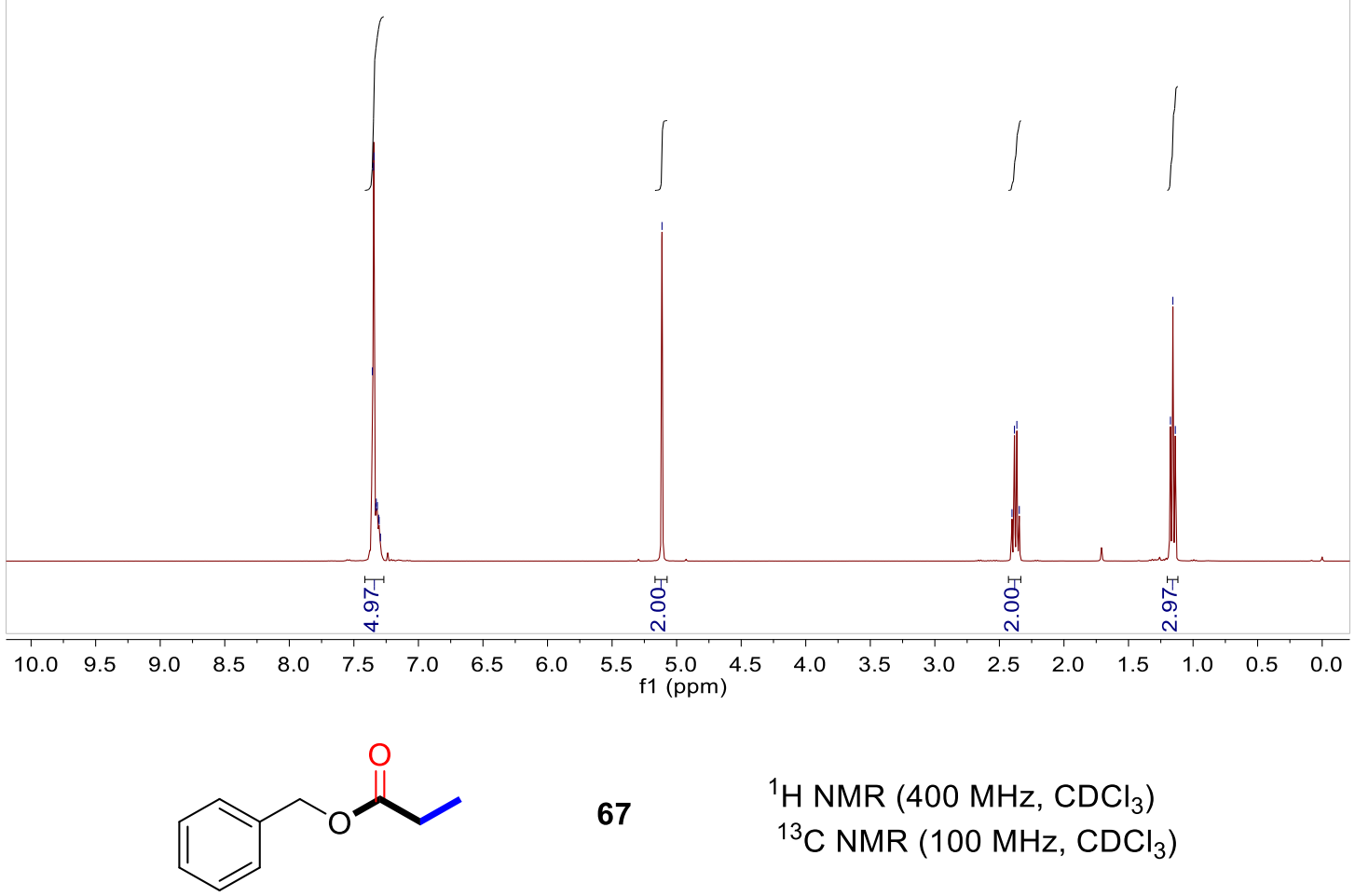

67

${ }^{1} \mathrm{H}$ NMR (400 MHz, $\left.\mathrm{CDCl}_{3}\right)$

${ }^{13} \mathrm{C} \mathrm{NMR}\left(100 \mathrm{MHz}, \mathrm{CDCl}_{3}\right)$

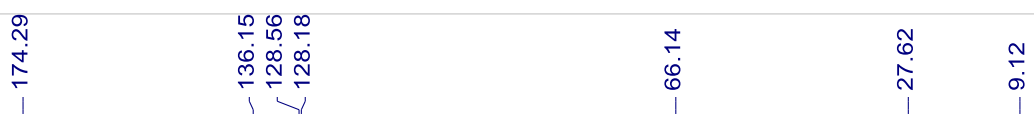

CSK-2-23-12.11.fid

C13CPD CDCI3 \{D:INMR400IDNL0604\} nmr-new 21

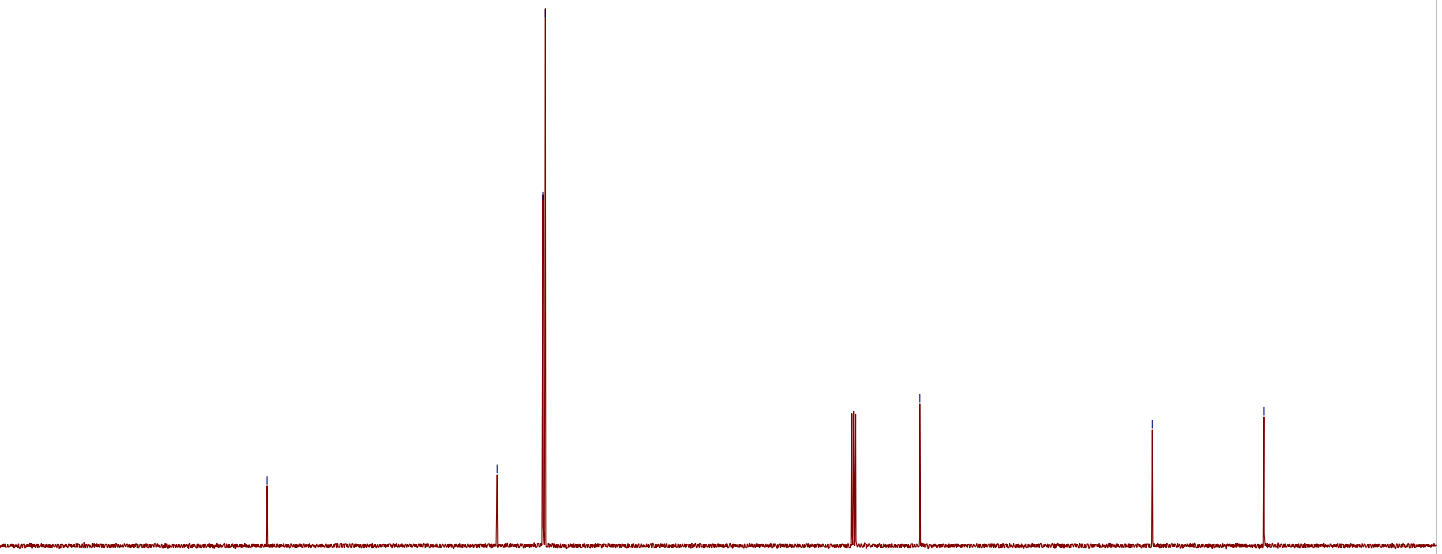

$\begin{array}{lllllllllllllllllllllll}210 & 200 & 190 & 180 & 170 & 160 & 150 & 140 & 130 & 120 & 110 & 100 & 90 & 80 & 70 & 60 & 50 & 40 & 30 & 20 & 10 & 0 & -10\end{array}$ f1 (ppm) 


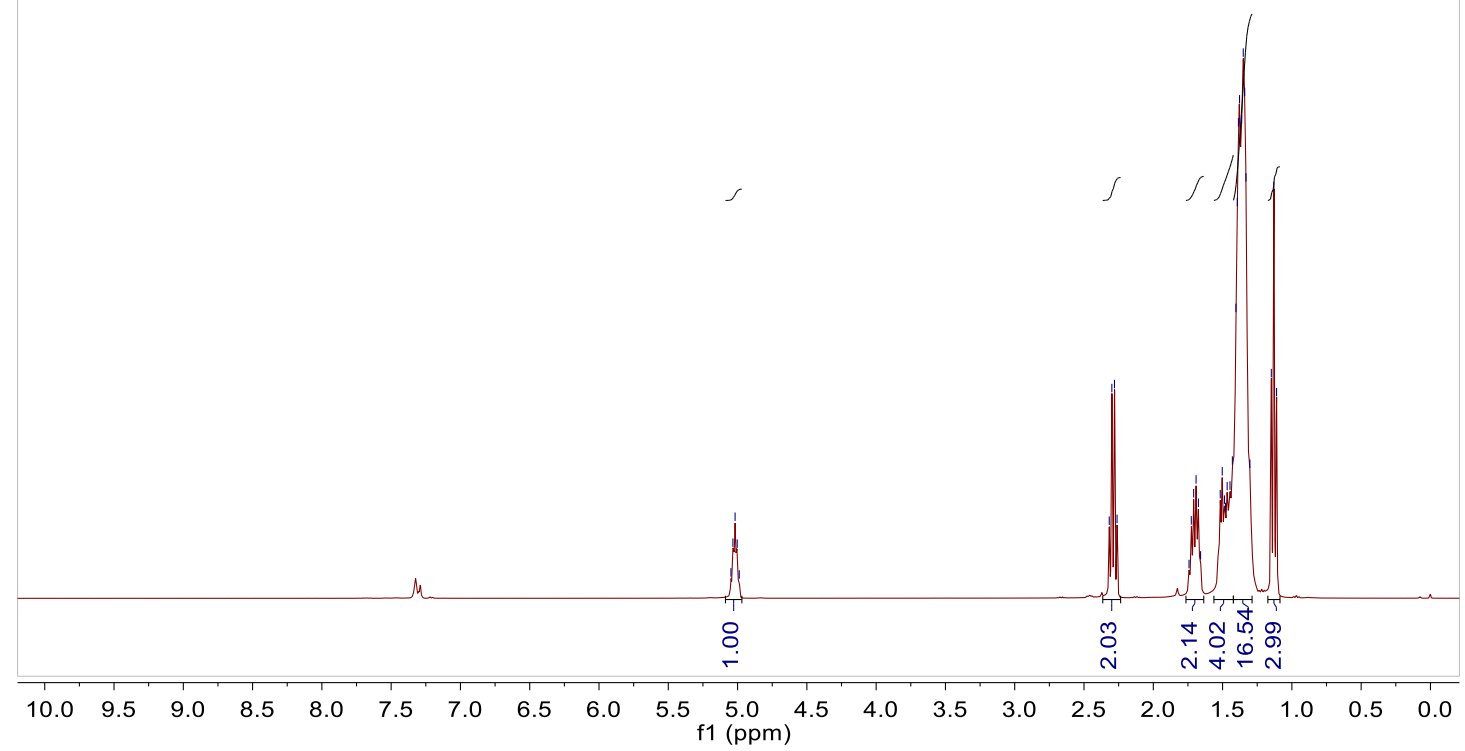

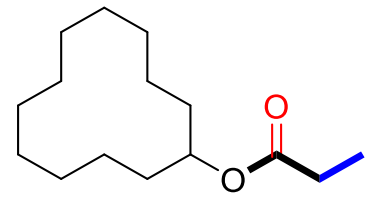

$\frac{i}{\stackrel{1}{n}}$

csk-3-33-7.11.fid

C13CPD CDCI3 \{D:INMR400IDNL0604\} nmr-new 20
$68 \quad{ }^{1} \mathrm{H}$ NMR $\left(400 \mathrm{MHz}, \mathrm{CDCl}_{3}\right)$

${ }^{13} \mathrm{C} \mathrm{NMR}\left(100 \mathrm{MHz}, \mathrm{CDCl}_{3}\right)$

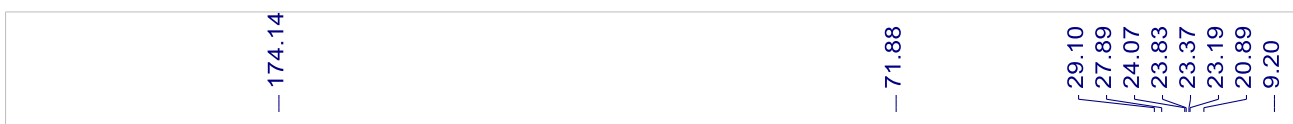

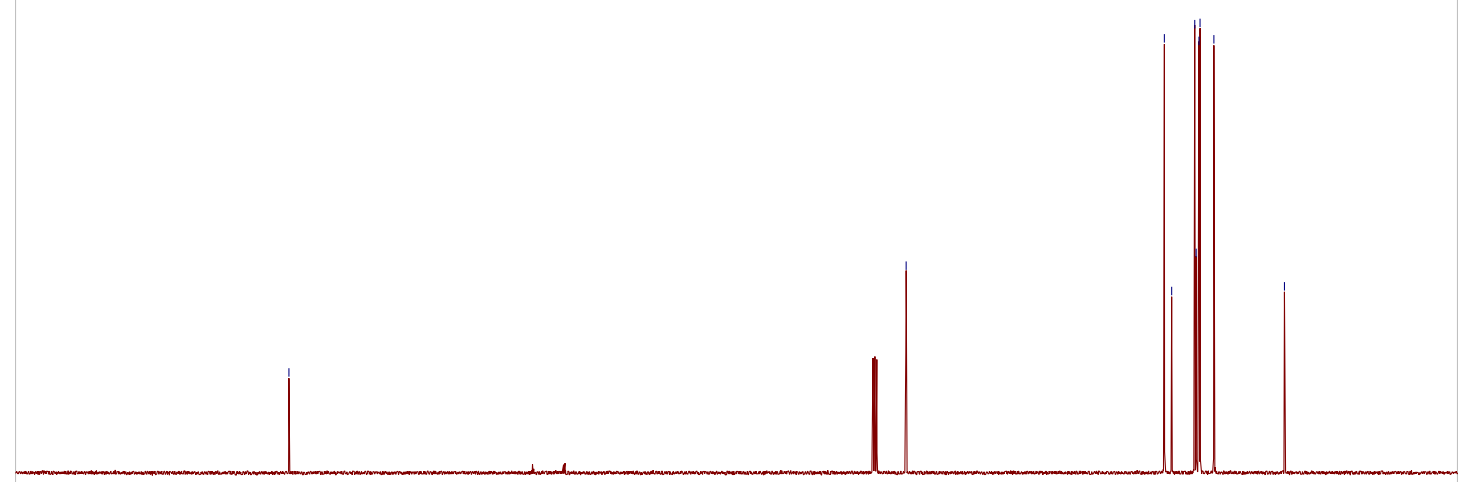

$\begin{array}{lllllllllllll}210 & 200 & 190 & 180 & 170 & 160 & 150 & 140 & 130 & 120 & 110 & 100 & 90\end{array}$ 


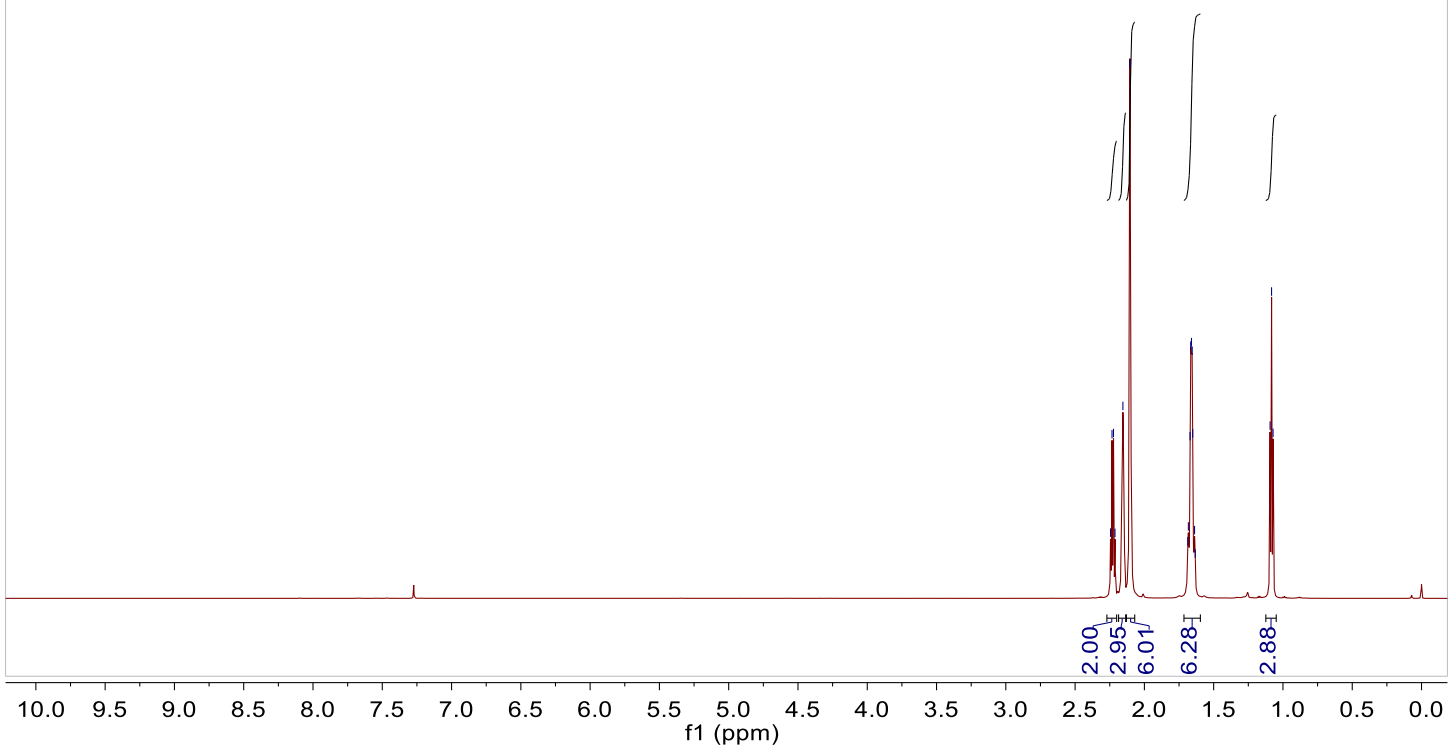

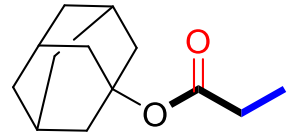

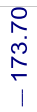

csk-3-33-16.11.fid

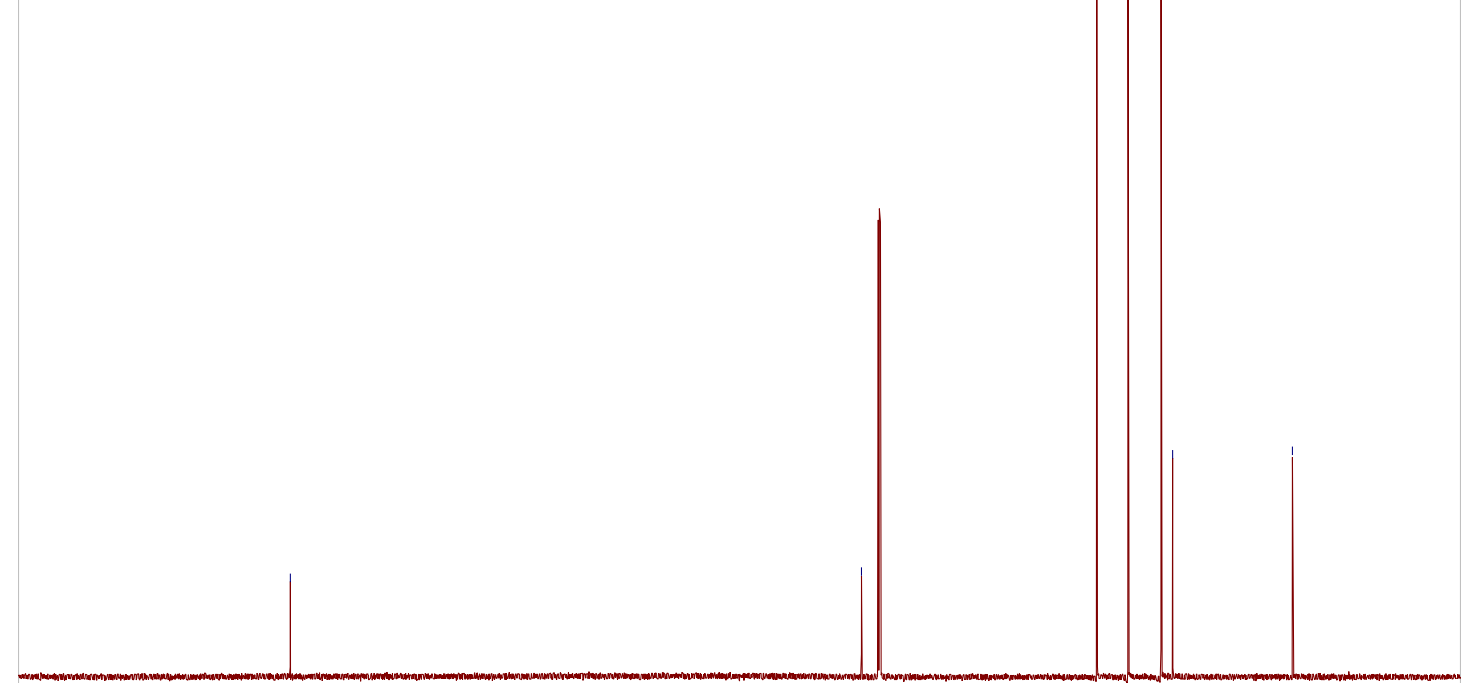

${ }^{1} \mathrm{H}$ NMR $\left(700 \mathrm{MHz}, \mathrm{CDCl}_{3}\right)$

${ }^{13} \mathrm{C}$ NMR $\left(176 \mathrm{MHz}, \mathrm{CDCl}_{3}\right)$

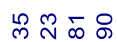

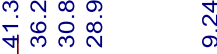

它

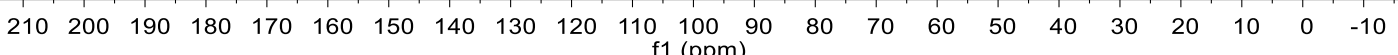




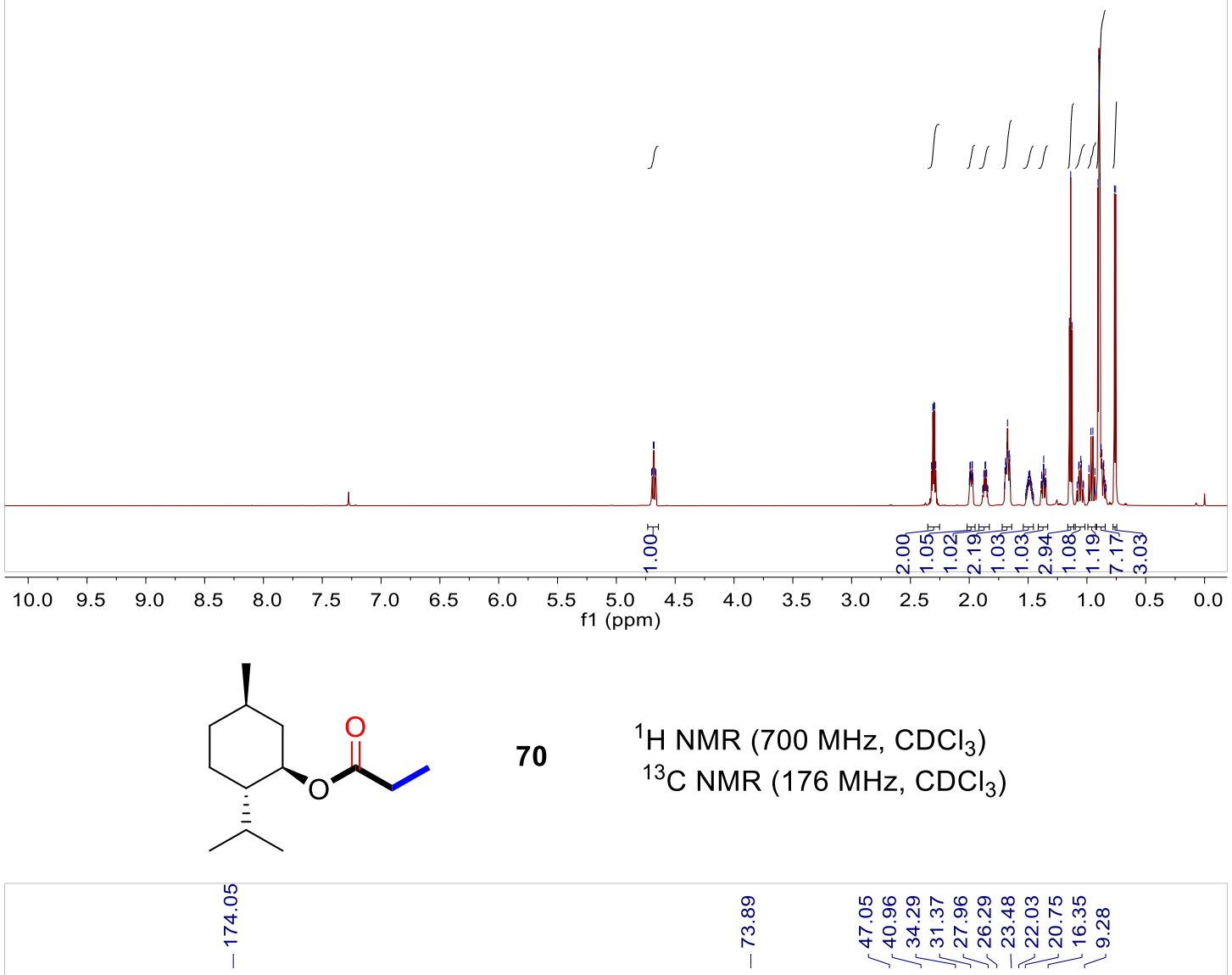

csk-3-33-17.22.fid

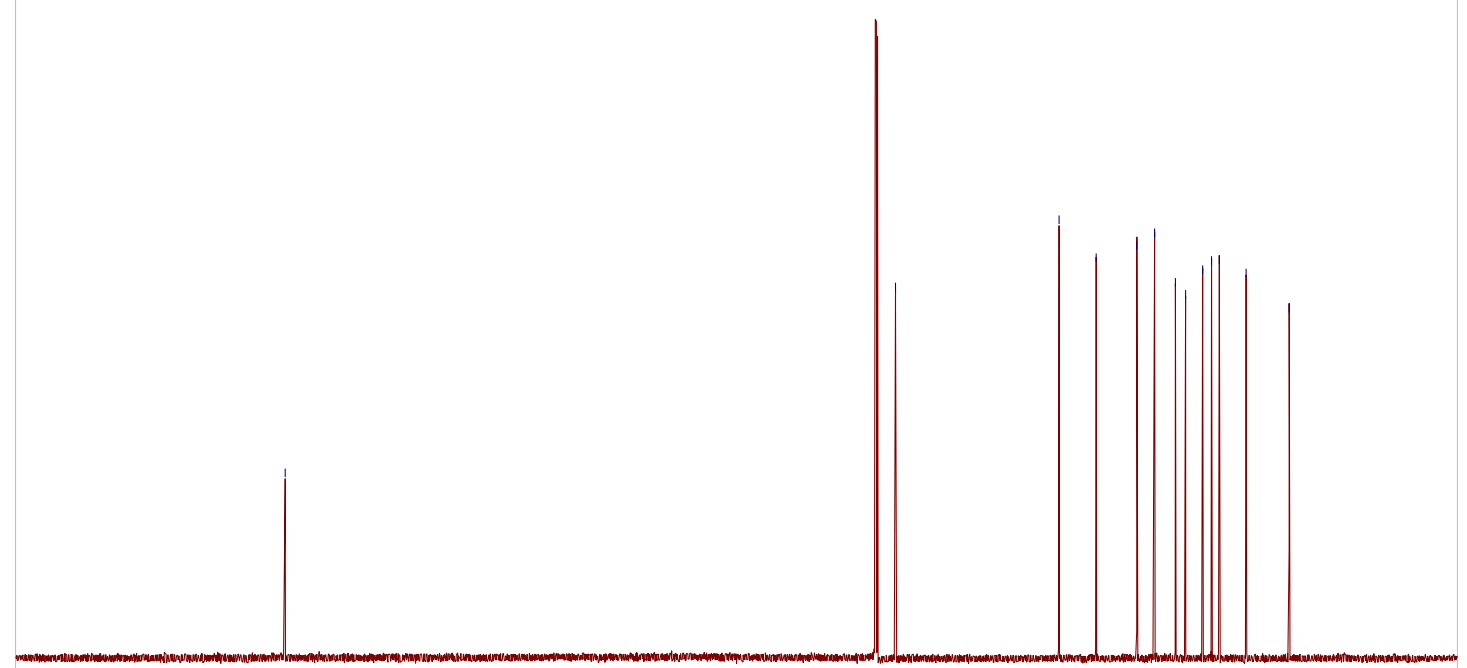

$\begin{array}{lllllllllllllllllllllll}210 & 200 & 190 & 180 & 170 & 160 & 150 & 140 & 130 & 120 & 110 & 100 & 90 & 80 & 70 & 60 & 50 & 40 & 30 & 20 & 10 & 0 & -10\end{array}$ f1 (ppm) 
csk-3-33-6.12.fid

PROTON CDCI3 \{D:INMR400ldnl0604\} nmr-new 49
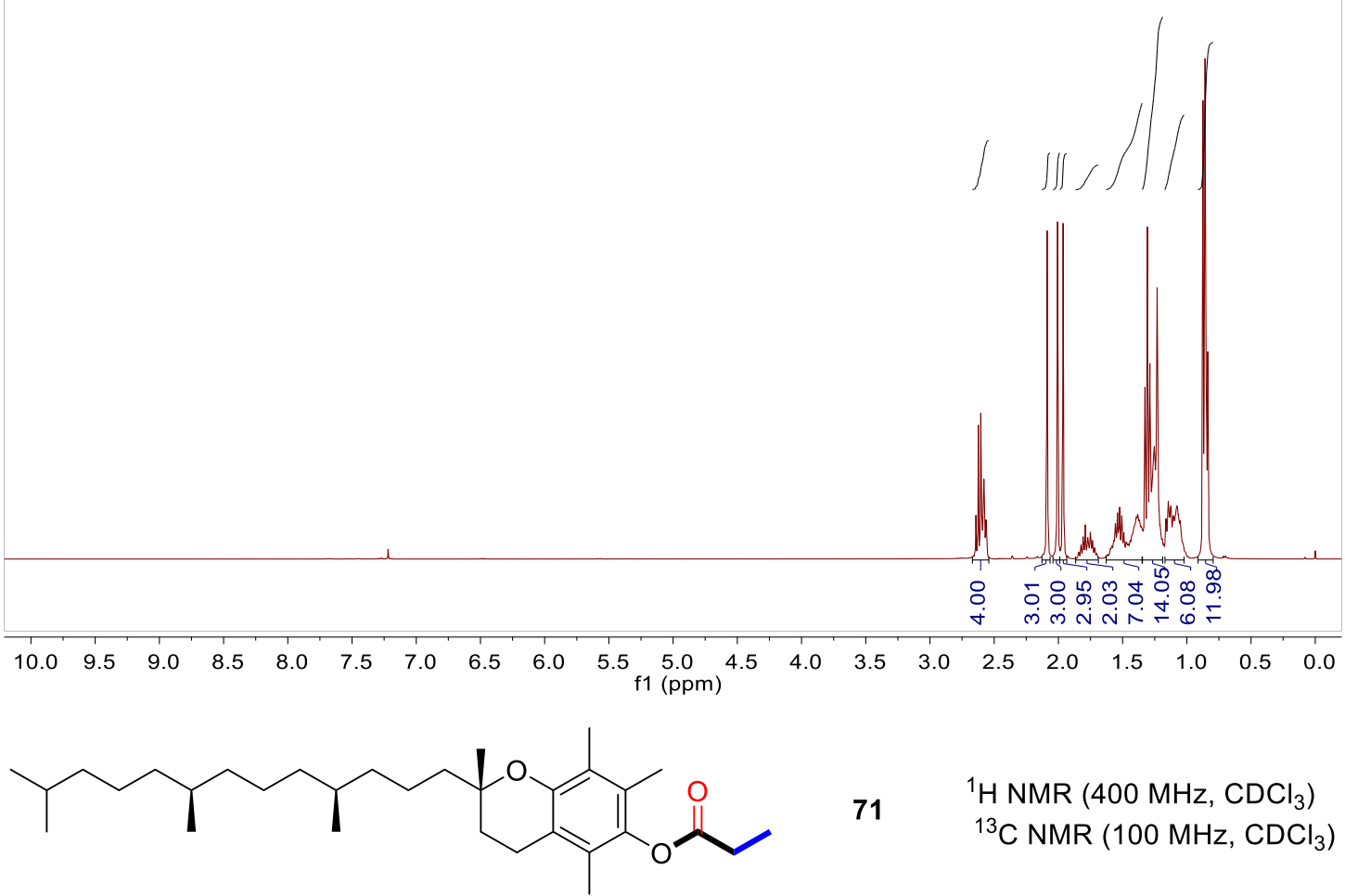

$71 \quad{ }^{1} \mathrm{H}$ NMR $\left(400 \mathrm{MHz}, \mathrm{CDCl}_{3}\right)$ ${ }^{13} \mathrm{C}$ NMR (100 $\left.\mathrm{MHz}, \mathrm{CDCl}_{3}\right)$

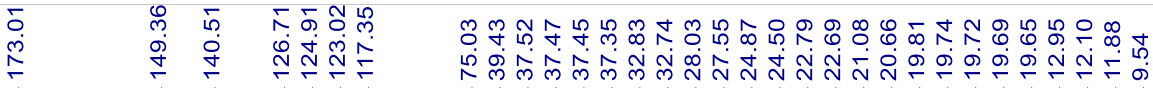

csk-3-33-6.13.fid

C13CPD CDCI3 \{D:INMR4001dn10604\} nmr-new 49

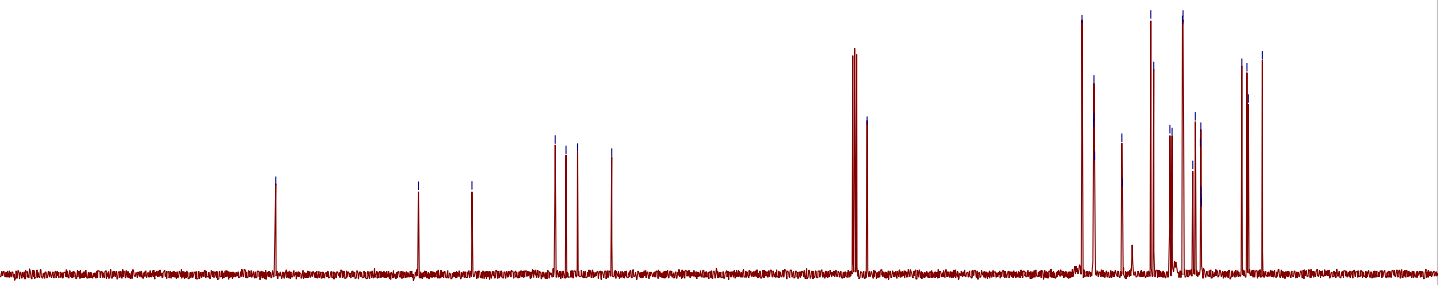

$\begin{array}{lllllllllllllllllllllll}210 & 200 & 190 & 180 & 170 & 160 & 150 & 140 & 130 & 120 & 110 & 100 & 90 & 80 & 70 & 60 & 50 & 40 & 30 & 20 & 10 & 0 & -10\end{array}$ 
CSK-3-33-10.10.fid

PROTON CDCI3 \{D:INMR400IDNL0604\} nmr-new 2
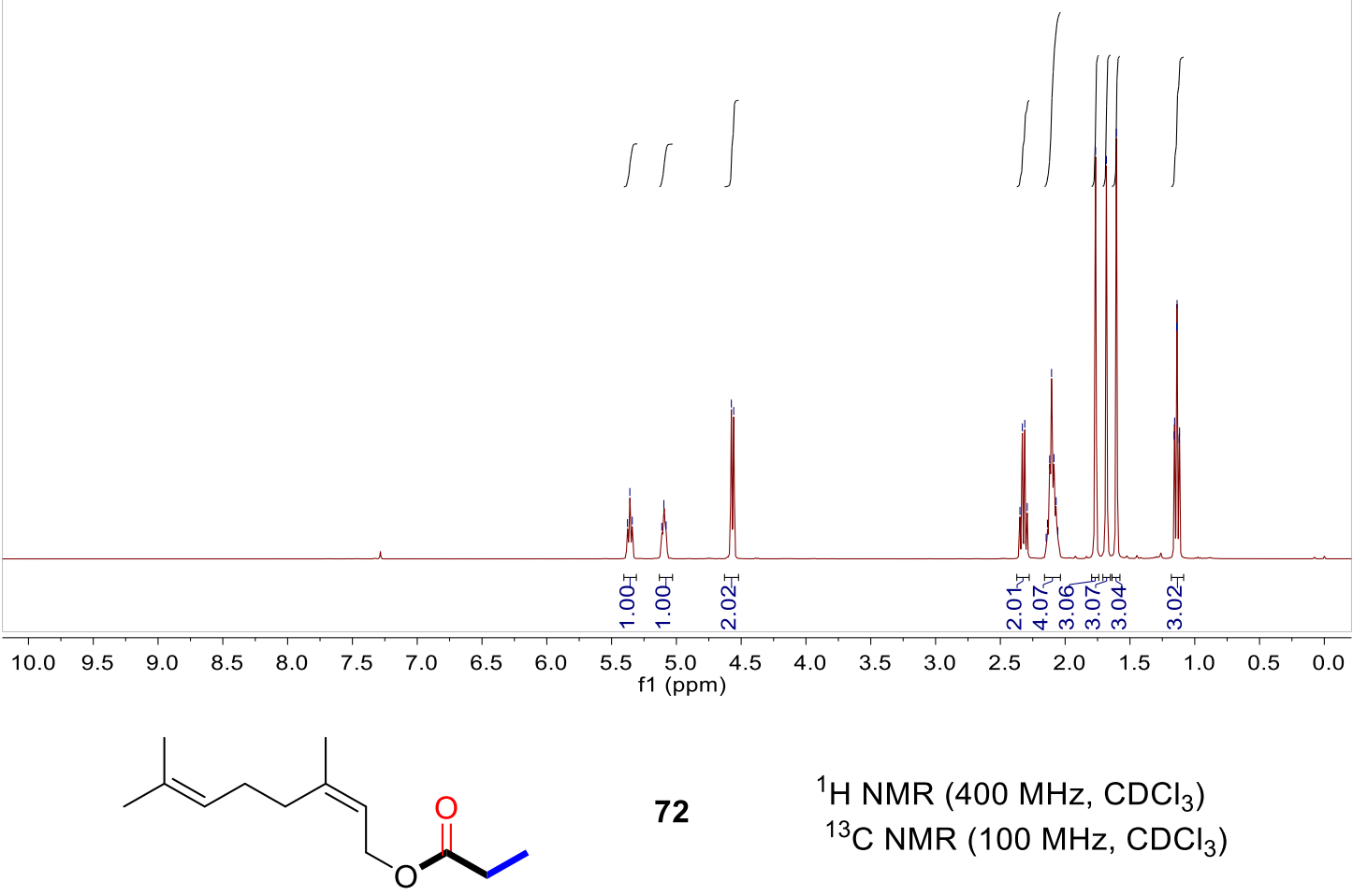

72

${ }^{1} \mathrm{H}$ NMR $\left(400 \mathrm{MHz}, \mathrm{CDCl}_{3}\right)$

${ }^{13} \mathrm{C}$ NMR $\left(100 \mathrm{MHz}, \mathrm{CDCl}_{3}\right)$

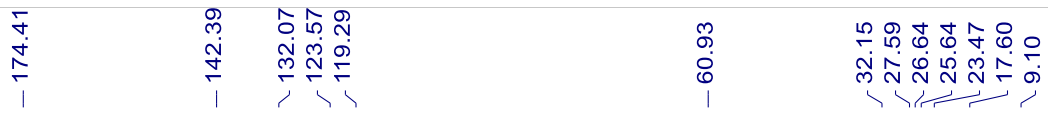

CSK-3-33-10.11. fid

C13CPD CDCI3 \{D:INMR400IDNL0604\} nmr-new 2

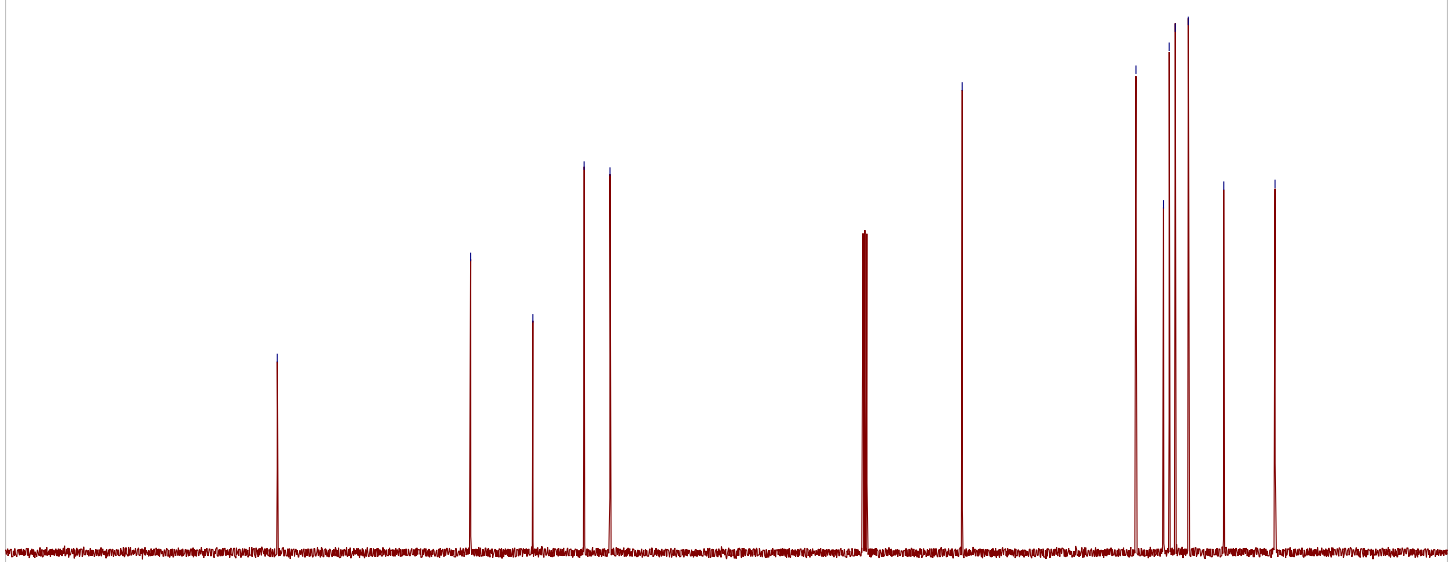

$\begin{array}{lllllllllllllllllllllll}210 & 200 & 190 & 180 & 170 & 160 & 150 & 140 & 130 & 120 & 110 & 100 & 90 & 80 & 70 & 60 & 50 & 40 & 30 & 20 & 10 & 0 & -10\end{array}$ 
csk-3-33-11.10.fid

PROTON CDCI3 \{D:INMR400IDNL0604\} nmr-new 21
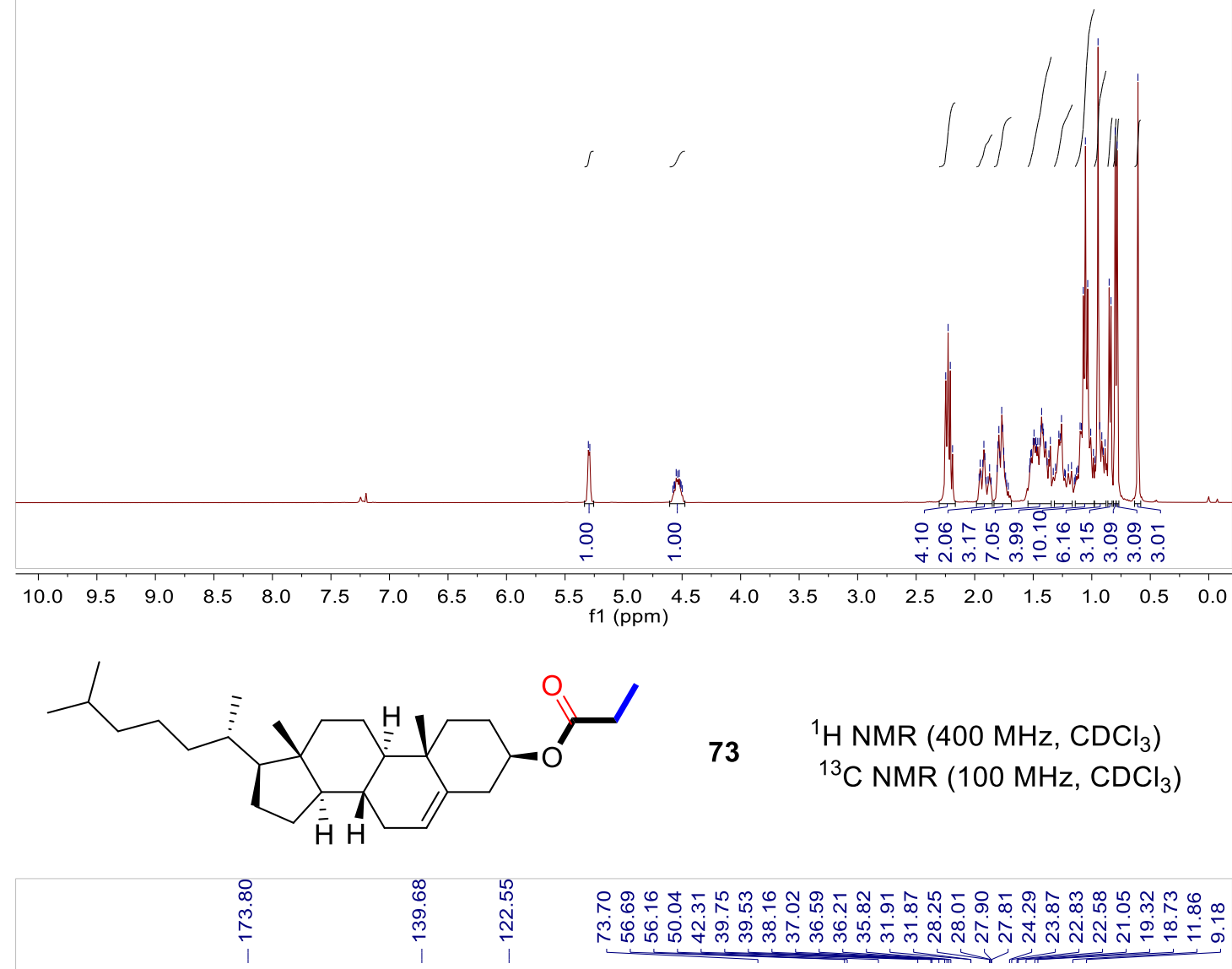

csk-3-33-11.11.fid

C13CPD CDCI3 \{D:INMR400IDNL0604\} nmr-new 21

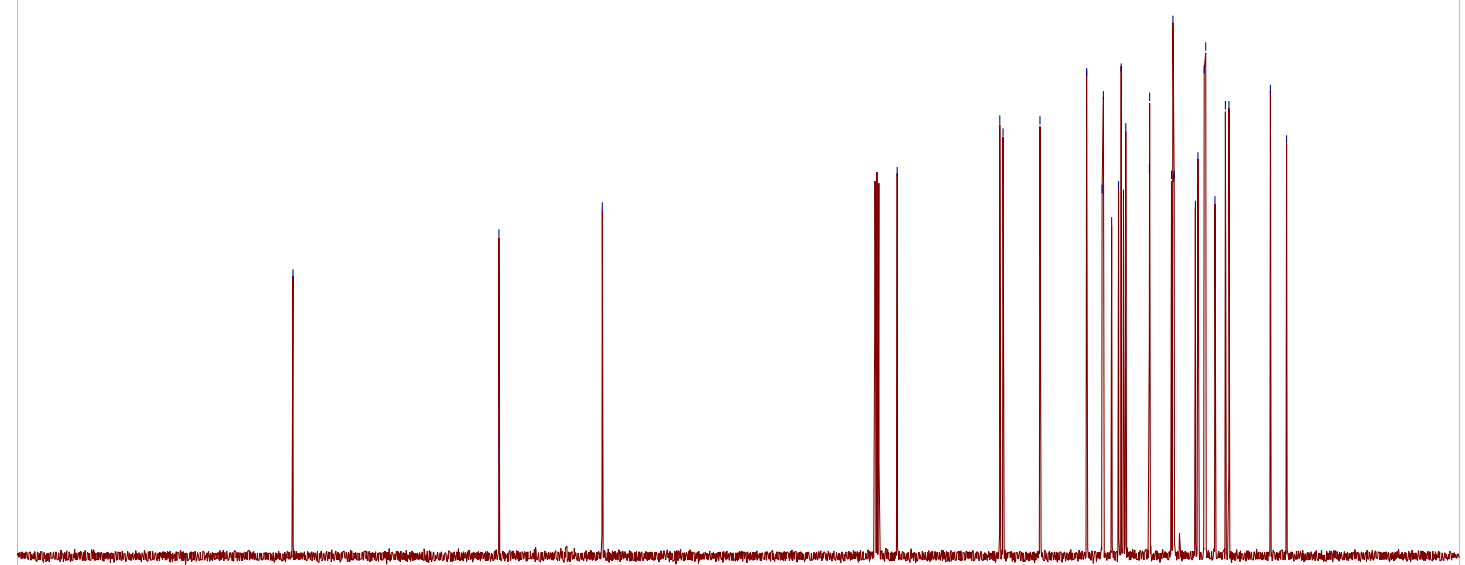

$\begin{array}{lllllllllllllllllllllll}210 & 200 & 190 & 180 & 170 & 160 & 150 & 140 & 130 & 120 & 110 & 100 & 90 & 80 & 70 & 60 & 50 & 40 & 30 & 20 & 10 & 0 & -10\end{array}$ f1 (ppm) 


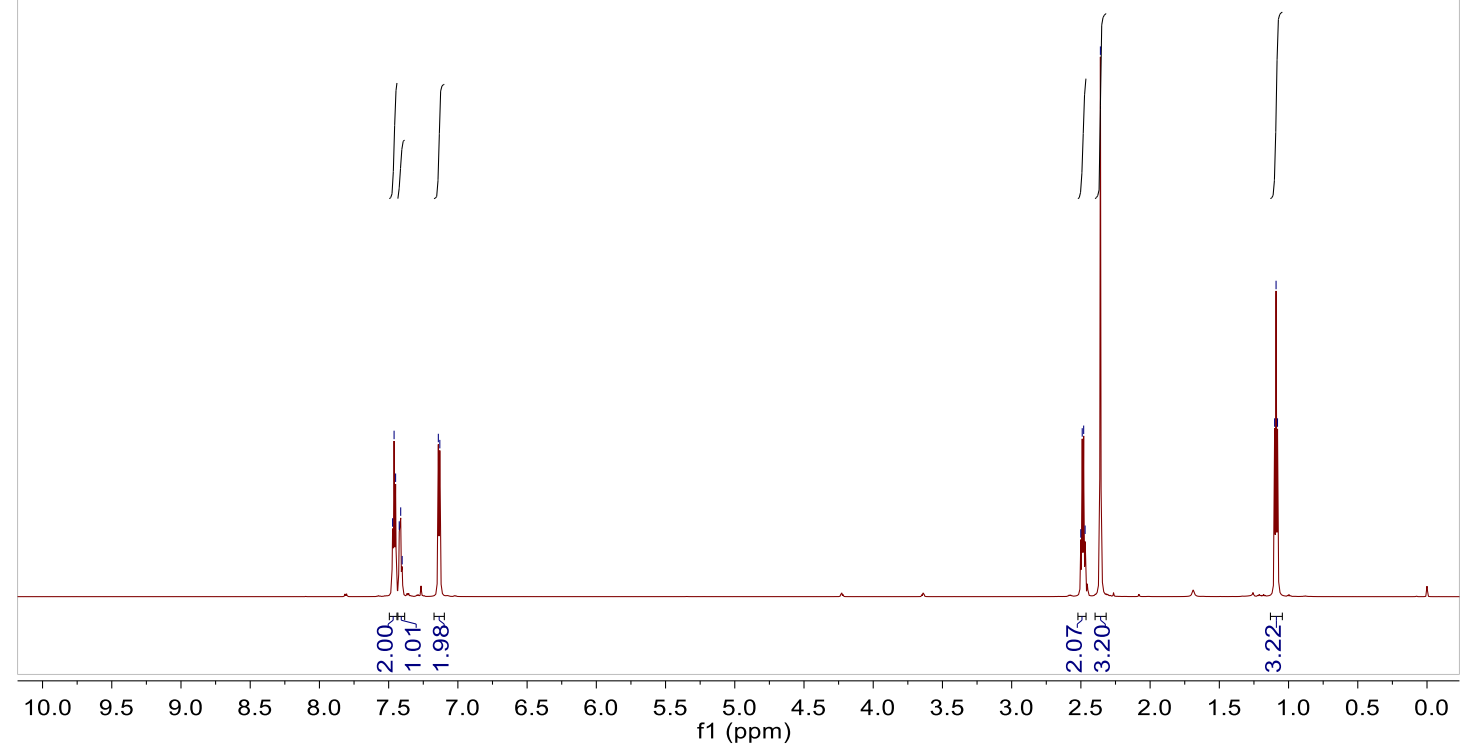<smiles>CCC(=O)N(C(C)=O)c1ccccc1</smiles>

\section{$74{ }^{1} \mathrm{H}$ NMR $\left(700 \mathrm{MHz}, \mathrm{CDCl}_{3}\right)$}

${ }^{13} \mathrm{C}$ NMR $\left(176 \mathrm{MHz}, \mathrm{CDCl}_{3}\right)$

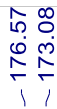

ำกㅇำ

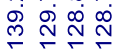

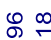

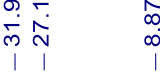

csk-3-42.11.fid

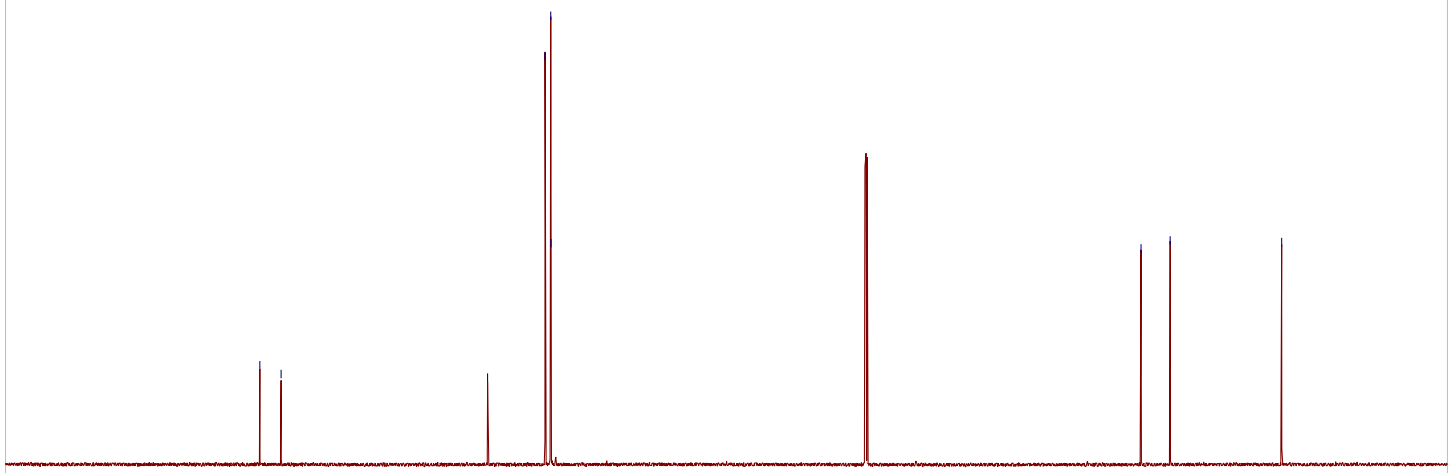

$\begin{array}{llllllllllllllllllllllll}210 & 200 & 190 & 180 & 170 & 160 & 150 & 140 & 130 & 120 & 110 & 100 & 90 & 80 & 70 & 60 & 50 & 40 & 30 & 20 & 10 & 0 & -10\end{array}$ 